\title{
C-H Amination Mediated by Cobalt Organoazide Adducts and the Corresponding Cobalt Nitrenoid Intermediates
}

\author{
Yunjung Baek ${ }^{\dagger}$ Anuvab Das, ${ }^{\ddagger}$ Shao-Liang Zheng, ${ }^{\dagger}$ Joseph H. Reibenspies, ${ }^{\ddagger}$ David C. Powers, ${ }^{\ddagger}$ \\ and Theodore A. Betley ${ }^{\dagger *}$ \\ †Department of Chemistry and Chemical Biology \\ Harvard University \\ 12 Oxford Street, Cambridge, Massachusetts 02138 \\ ‡Department of Chemistry \\ 3255 TAMU, Texas A\&M University \\ College Station, Texas 77843
}

Page

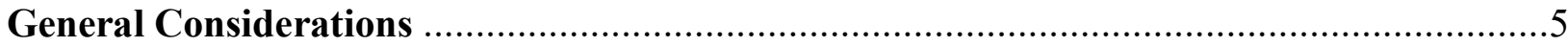

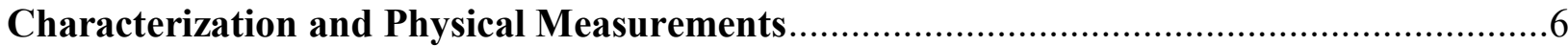

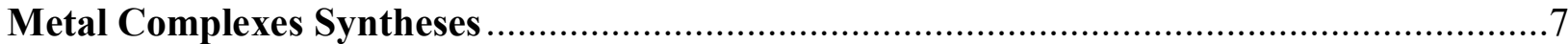

Non-Covalent Interaction Analysis ...........................................................................15

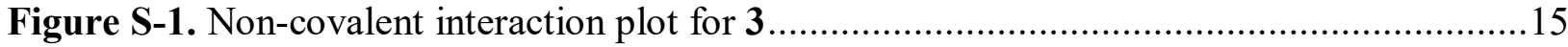

Stoichiometric Reactions............................................................................................

Reaction with cyclohexene.

Reaction with styrene

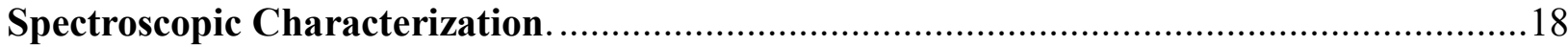

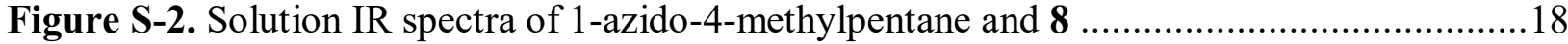

Figure S-3. Solution IR spectra of (4-azidobutyl)benzene and 15..........................................18

Figure S-4. Solution IR spectra of 1-azido-4-methylpentane and $\mathbf{1 7}$ in benzene in the presence of a slight excess amount of 1-azido-4-methylpentane............................................................. 19

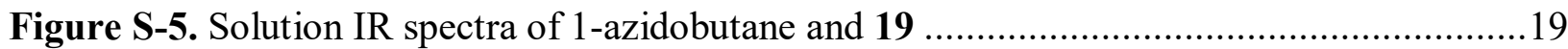

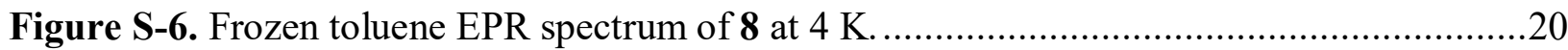

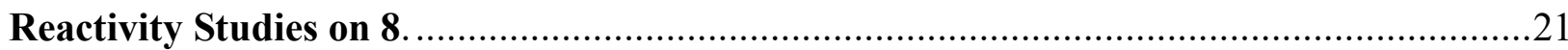

Figure S-7. Reaction progression for the conversion of $\mathbf{8}$ to the corresponding imine complex .21

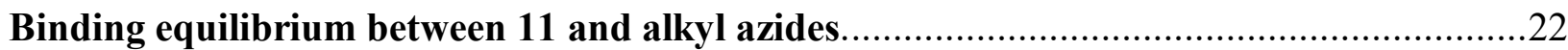


Figure S-8. $600 \mathrm{MHz}{ }^{1} \mathrm{H}$ NMR spectra of 17-Br at varying concentrations of 1-azido-4-

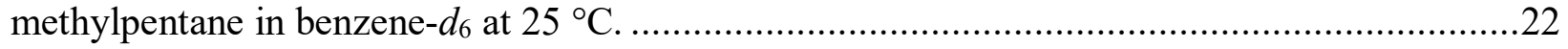

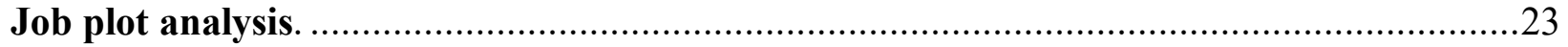

Figure S-9. Job plot obtained at $25^{\circ} \mathrm{C}$ in benzene- $d_{6}$ with $[\mathrm{Co}]+\left[\mathrm{RN}_{3}\right]=8.85 \mathrm{mM}$.............23

Table S-1. Chemical shifts (ppm) of $\mathbf{1 1}$ as a function of $\left[\mathrm{RN}_{3}\right]$ at $25{ }^{\circ} \mathrm{C}$ in benzene- $d_{6} \ldots \ldots \ldots \ldots . . .23$

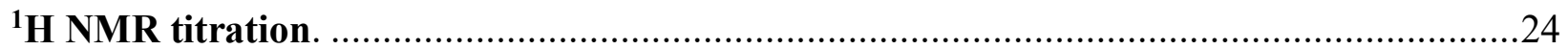

Figure S-10. $600 \mathrm{MHz}{ }^{1} \mathrm{H}$ NMR titration curves of 11 with organoazides..........................24

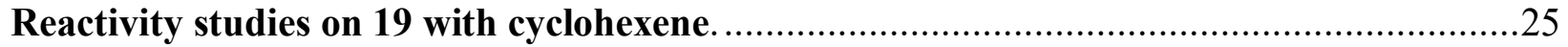

Figure S-11. Reaction progression for the conversion of 19 to 21 in benzene- $d_{6}$ at $40{ }^{\circ} \mathrm{C} \ldots \ldots \ldots . .25$

${ }^{1}$ H NMR Spectra of Metal Compounds ..................................................................26

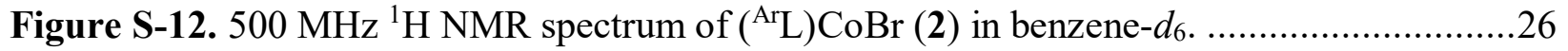

Figure S-13. $500 \mathrm{MHz}{ }^{1} \mathrm{H}$ NMR spectrum of $\left({ }^{\mathrm{Ar}} \mathrm{L}\right) \mathrm{CoBr}\left(\mathrm{N}_{3}\left(\mathrm{C}_{6} \mathrm{H}_{4}-p-{ }^{t} \mathrm{Bu}\right)\right)(3)$ in benzene- $d_{6} \ldots . .26$

Figure S-14. $500 \mathrm{MHz}{ }^{1} \mathrm{H}$ NMR spectrum of ( $\left.{ }^{\mathrm{Ar}} \mathrm{L}\right) \mathrm{CoBr}\left([3+2]\right.$ annulation) (4) in benzene- $d_{6} \ldots .27$

Figure S-15. $500 \mathrm{MHz}{ }^{1} \mathrm{H}$ NMR spectrum of ( $\left.{ }^{\mathrm{Ar}} \mathrm{L}\right) \mathrm{CoBr}\left(\mathrm{NH}_{2}\left(\mathrm{C}_{6} \mathrm{H}_{4}-p-{ }^{t} \mathrm{Bu}\right)\right)(5)$ in benzene- $d_{6} .27$

Figure S-16. $500 \mathrm{MHz}{ }^{1} \mathrm{H}$ NMR spectra of $\left({ }^{\mathrm{Ar}} \mathrm{L}\right) \mathrm{CoBr}\left(\mathrm{N}_{3} \mathrm{CH}_{2} \mathrm{R}\right)\left(\mathbf{6}, \mathbf{7}\right.$, and 8) in benzene- $d_{6} \ldots . .28$

Figure S-17. $500 \mathrm{MHz}{ }^{1} \mathrm{H}$ NMR spectrum of ( $\left.{ }^{\mathrm{Ar}} \mathrm{L}\right) \mathrm{CoBr}\left(\mathrm{NHCHC}_{6} \mathrm{H}_{5}\right)(9)$ in benzene- $d_{6} \ldots \ldots \ldots . .29$

Figure S-18. $500 \mathrm{MHz}{ }^{1} \mathrm{H}$ NMR spectra of (a) $\left[\left({ }^{t \mathrm{Bu}} \mathrm{L}\right) \mathrm{CoCl}\right]_{2}(\mathbf{1 0})$ and $\left.(\mathrm{b})\left[{ }^{t \mathrm{Bu}} \mathrm{L}\right) \mathrm{CoBr}\right]_{2}(\mathbf{1 1})$ in

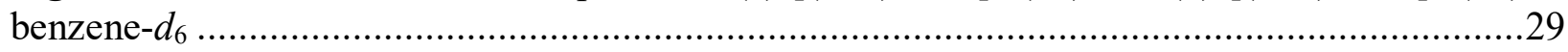

Figure S-19. $500 \mathrm{MHz}{ }^{1} \mathrm{H}$ NMR spectrum of $\left({ }^{t \mathrm{Bu}} \mathrm{L}\right) \mathrm{CoBr}(\mathrm{thf})(\mathbf{1 2})$ in benzene- $d_{6} \ldots \ldots \ldots \ldots \ldots \ldots . . . . . . . .30$

Figure S-20. $500 \mathrm{MHz}{ }^{1} \mathrm{H}$ NMR spectrum of $\left({ }^{t \mathrm{Bu}} \mathrm{L}\right) \mathrm{CoCl}(1,2,3$-dihydrotriazole) (14) in benzene-

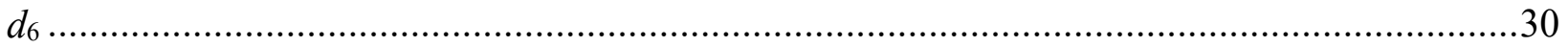

Figure S-21. $500 \mathrm{MHz}{ }^{1} \mathrm{H}$ NMR spectra of $\left({ }^{t \mathrm{Bu}} \mathrm{L}\right) \mathrm{CoCl}\left(\mathrm{N}_{3} \mathrm{R}\right)\left(\mathbf{1 5}, \mathbf{1 7}\right.$, and 19) in benzene-d $d_{6} \ldots . .31$

Figure S-22. $500 \mathrm{MHz}{ }^{1} \mathrm{H}$ NMR spectrum of $\left({ }^{t \mathrm{Bu}} \mathrm{L}\right) \mathrm{CoCl}\left(2\right.$-phenylpyrrolidine) (16) in benzene- $d_{6}$ 32

Figure S-23. $500 \mathrm{MHz}{ }^{1} \mathrm{H}$ NMR spectrum of $\left({ }^{t \mathrm{Bu}} \mathrm{L}\right) \mathrm{CoCl}(2,2-$ dimethylpyrrolidine) (18) in

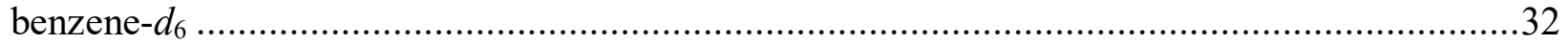

Figure S-24. $500 \mathrm{MHz}{ }^{1} \mathrm{H}$ NMR spectrum of $\left({ }^{t \mathrm{Bu}} \mathrm{L}\right) \mathrm{CoCl}$ (butan-1-imine) (20) in benzene- $d_{6} \ldots .33$

Figure S-25. $500 \mathrm{MHz}{ }^{1} \mathrm{H}$ NMR spectrum of $\left({ }^{t \mathrm{Bu}} \mathrm{L}\right) \mathrm{CoCl}\left(n-\mathrm{BuNH}_{2}\right)(\mathbf{2 1})$ in benzene-d $d_{6} \ldots \ldots \ldots . . .33$

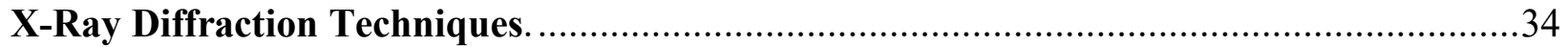

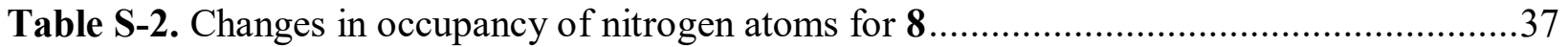

Figure S-26. Difference density map obtained for complex 8 at $100 \mathrm{~K}$ following four iterations of

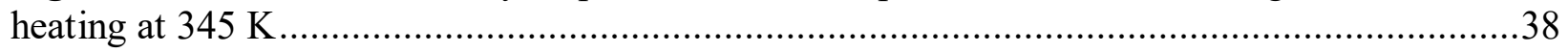

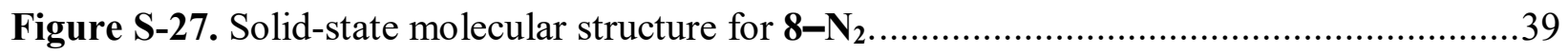

Figure S-28. Changes in occupancy of the organoazide $\mathrm{N}$-atoms in 7 during synchrotron irradiation $(\lambda=0.41328 \AA)$. . 40 
Figure S-29. Changes in occupancy of the organoazide $\mathrm{N}$-atoms in 8 during synchrotron

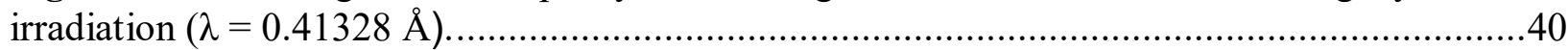

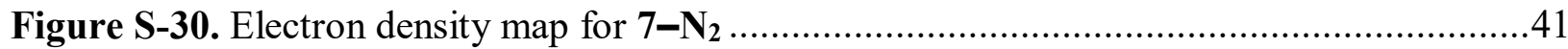

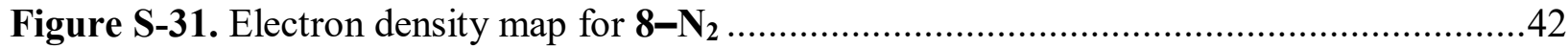

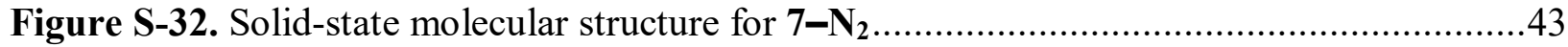

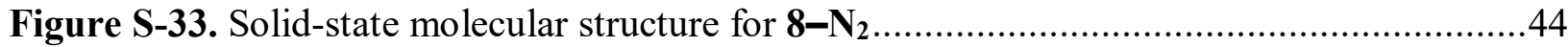

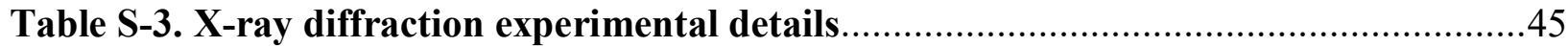

Figure S-34. Solid-state molecular structure for $\left({ }^{\mathrm{Ar}} \mathrm{L}\right) \mathrm{CoBr}\left(\mathrm{N}_{3}\left(\mathrm{C}_{6} \mathrm{H}_{4}-p-{ }^{t} \mathrm{Bu}\right)\right)(\mathbf{3}) \ldots \ldots \ldots \ldots \ldots \ldots \ldots . . .49$

Figure S-35. Solid-state molecular structure for $\left({ }^{\mathrm{Ar}} \mathrm{L}\right) \mathrm{CoBr}([3+2]$ annulation $)(4)$......................50

Figure S-36. Solid-state molecular structure for $\left({ }^{\mathrm{Ar}} \mathrm{L}\right) \mathrm{CoBr}\left(\mathrm{NH}_{2}\left(\mathrm{C}_{6} \mathrm{H}_{4}-p-{ }^{\mathrm{t}} \mathrm{Bu}\right)\right)(\mathbf{5}) \ldots \ldots \ldots \ldots \ldots . . . .51$

Figure S-37. Solid-state molecular structure for $\left({ }^{\mathrm{Ar}} \mathrm{L}\right) \mathrm{CoBr}\left(\mathrm{N}_{3} \mathrm{R}\right)(7) \ldots \ldots \ldots \ldots \ldots \ldots \ldots \ldots \ldots \ldots \ldots . . .52$

Figure S-38. Solid-state molecular structure for $\left({ }^{\mathrm{Ar}} \mathrm{L}\right) \mathrm{CoBr}\left(\mathrm{N}_{3} \mathrm{R}\right)(\mathbf{8}) \ldots \ldots \ldots \ldots \ldots \ldots \ldots \ldots \ldots \ldots \ldots \ldots . . .53$

Figure S-39. Solid-state molecular structure for $\left({ }^{\mathrm{Ar}} \mathrm{L}\right) \mathrm{CoBr}\left(\mathrm{NHCHC}_{6} \mathrm{H}_{5}\right)(\mathbf{9}) \ldots \ldots \ldots \ldots \ldots \ldots \ldots \ldots . . . .54$

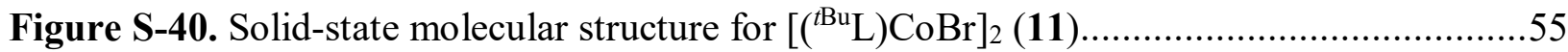

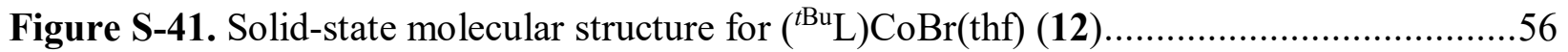

Figure S-42. Solid-state molecular structure for $\left.{ }^{\left({ }^{\mathrm{Bu}} \mathrm{L}\right.}\right) \mathrm{CoBr}(1,2,3$-dihydrotriazole) $(\mathbf{1 4}-\mathrm{Br}) \ldots . . .57$

Figure S-43. Solid-state molecular structure for $\left({ }^{t \mathrm{Bu}} \mathrm{L}\right) \mathrm{CoBr}(2$-phenylpyrrolidine) $(\mathbf{1 6}-\mathrm{Br}) \ldots \ldots . . .58$

Figure S-44. Solid-state molecular structure for $\left.{ }^{\left({ }^{\mathrm{Bu}} \mathrm{L}\right.} \mathrm{L}\right) \mathrm{CoCl}(2,2$-dimethylpyrrolidine) (18) ........59

Figure S-45. Solid-state molecular structure for $\left.\left({ }^{t \mathrm{Bu}} \mathrm{L}\right) \mathrm{CoCl}\left(\mathrm{NHCH}_{(\mathrm{CH}}\right) \mathrm{CH}_{3}\right)(\mathbf{2 0})$.................60

Figure S-46. Solid-state molecular structure for $\left({ }^{t \mathrm{Bur}} \mathrm{L}\right) \mathrm{CoCl}\left(n-\mathrm{BuNH}_{2}\right)(\mathbf{2 1}) \ldots \ldots \ldots \ldots \ldots \ldots \ldots \ldots \ldots . . .61$

Computational Methods

Table S-4. Summary of the geometry optimized results of $\mathbf{8}-\mathbf{N}_{2}$ as Co(IV) imido at the $S=1 / 2$, $3 / 2$, and $5 / 2$ spin states

Table S-5. Summary of the broken-symmetry geometry optimized results of $\mathbf{8}-\mathbf{N}_{\mathbf{2}}$ as $\mathrm{Co}$ (III) iminyl at the $S=1$ and $S=2$ spin states

Figure S-47. Geometry optimized molecular structure and Mulliken spin density plot $(\alpha-\beta)$ of $\mathbf{8}-\mathbf{N}_{\mathbf{2}}$ at an $S=1 / 2$ spin state of Co(IV). .65

Figure S-48. Geometry optimized molecular structure and Mulliken spin density plot $(\alpha-\beta)$ of $\mathbf{8}-\mathbf{N}_{2}$ at an $S=3 / 2$ spin state of Co(IV).

Figure S-49. Geometry optimized molecular structure and Mulliken spin density plot $(\alpha-\beta)$ of $\mathbf{8}-\mathbf{N}_{2}$ at an $S=5 / 2$ spin state of Co(IV). .66

Figure S-50. Geometry optimized molecular structure and Mulliken spin density plot $(\alpha-\beta)$ of $\mathbf{8}-\mathbf{N}_{2}$ at an $S=1$ spin state of $\mathrm{Co}(\mathrm{III})$.

Figure S-51. Geometry optimized molecular structure and Mulliken spin density plot $(\alpha-\beta)$ of $\mathbf{8}-\mathbf{N}_{2}$ at an $S=2$ spin state of Co(III)

Table S-6. Coordinates of optimized molecular structure for the truncated Co-nitrenoid $\left(\mathbf{8}-\mathbf{N}_{2}\right)$ at low-spin Co(IV) state. 
Table S-7. Coordinates of optimized molecular structure for the truncated Co-nitrenoid $\left(\mathbf{8}-\mathbf{N}_{2}\right)$ at

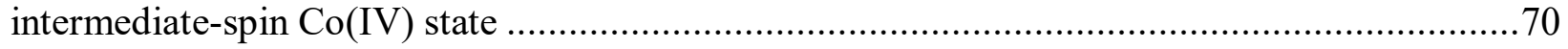

Table S-8. Coordinates of optimized molecular structure for the truncated Co-nitrenoid $\left(\mathbf{8}-\mathbf{N}_{2}\right)$ at

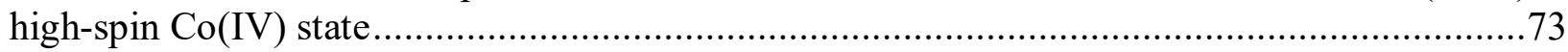

Table S-9. Coordinates of optimized molecular structure for the truncated Co-nitrenoid $\left(\mathbf{8}-\mathbf{N}_{2}\right)$ at

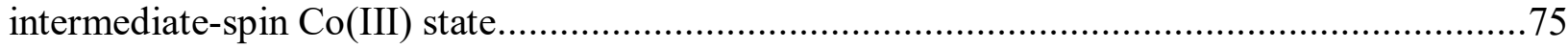

Table S-10. Coordinates of optimized molecular structure for the truncated Co-nitrenoid $\left(\mathbf{8}-\mathbf{N}_{2}\right)$

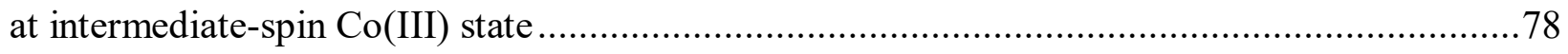

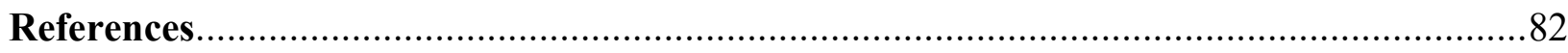

CheckCIF reports for crystallographic data ........................................................... 85 


\section{General Considerations.}

All manipulations of metal complexes were carried out in the absence of water and dioxygen using standard Schlenk techniques, or in an MBraun inert atmosphere drybox under a dinitrogen atmosphere. Ligand and ligand precursors were synthesized as previously reported. ${ }^{1}$ $\left({ }^{\mathrm{Ar}} \mathrm{L}\right) \mathrm{CoCl}(\mathrm{py})(\mathbf{1})^{2}$ and $\left({ }^{\mathrm{Ar}} \mathrm{L}\right) \mathrm{CoBr}(\mathbf{2})^{3}$ were prepared as previously reported. All glassware was oven dried for a minimum of 1 hour and cooled in an evacuated antechamber prior to use in the drybox. Benzene, diethyl ether, $n$-hexane, toluene, pentane, dichloromethane, and tetrahydrofuran were dried over $4 \AA$ molecular sieves (Strem) prior to use. Chloroform- $d$ was purchased from Cambridge Isotope Labs and used as received. Benzene- $d_{6}$ was purchased from Cambridge Isotope Labs, degassed, and stored over $4 \AA$ molecular sieves prior to use. 4-(tert-butyl)aniline, 2,4,6trifluoroaniline, 2,3,4,5,6-pentafluoroaniline, sodium azide, sodium nitrite, tert-butyl nitrite, 1,4cyclohexadiene, styrene, and trifluoroacetic acid were purchased from Aldrich. All C-H substrates were dried over calcium hydride and distilled under nitrogen or vacuum. 1-Azido-4-(tertbutyl)benzene was synthesized following published literature procedures. ${ }^{4}$ 1-Azido-2,3,4,5,6pentafluorobenzene and 2-azido-1,3,5-trifluorobenzene were synthesized following published literature procedure. ${ }^{5}$ (Azidomethyl)benzene was purchased from Alfa Aesar and used as received. 1-Azidobutane, 1-azido-4-methylpentane, (4-azidobutyl)benzene, and 6-azidohex-1-ene were prepared as previously reported. ${ }^{6}$ Anhydrous cobalt(II) bromide was purchased from Aldrich and used as received. Celite ${ }^{\circledR} 545$ (J. T. Baker) was dried in a Schlenk flask for $24 \mathrm{~h}$ under dynamic vacuum while heating to at least $150{ }^{\circ} \mathrm{C}$ prior to use in a drybox. Silica gel 32-63 $\mu$ (AIC, Framingham, MA) was used as received. 


\section{Characterization and Physical Measurements.}

${ }^{1} \mathrm{H}$ NMR spectra were recorded on Varian Unity/Inova $500 \mathrm{MHz}$ or Agilent DD2 $600 \mathrm{MHz}$ spectrometers. ${ }^{1} \mathrm{H}$ NMR chemical shifts are reported relative to $\mathrm{SiMe}_{4}$ using the chemical shift of residual solvent peaks as reference. Gas chromatography/mass spectrometry (GC/MS) was performed on an Agilent GC/MS 5975 Turbo system. EPR spectra were obtained on a Bruker EleXsys E-500 CW-EPR spectrometer. Spectra were measured as frozen toluene glasses at liquid $\mathrm{He}$ temperatures at a microwave power of $0.6325-2 \mathrm{~mW}$. Spectral simulations incorporating spin state, rhombicity, and zero field splitting were performed using EasySpin. ${ }^{7}$ UV/visible spectra were recorded on a Varian Cary $50 \mathrm{UV} /$ visible spectrometer using a $0.1 \mathrm{~cm}$ quartz cuvette and a scan rate of $600 \mathrm{~nm} / \mathrm{min}$. Elemental analyses were carried out on a Perkin Elmer 2400 Series II CHNS/O analyzer. IR spectra were obtained on a Varian Scimitar 1000 FTIR spectrometer using a $\mathrm{KBr}$ liquid IR-cell with a path length of $0.1 \mathrm{~mm}$. 


\section{Metal Complexes Syntheses.}

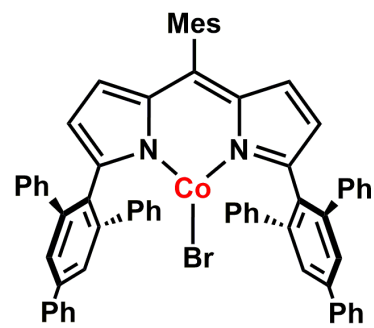

(2)

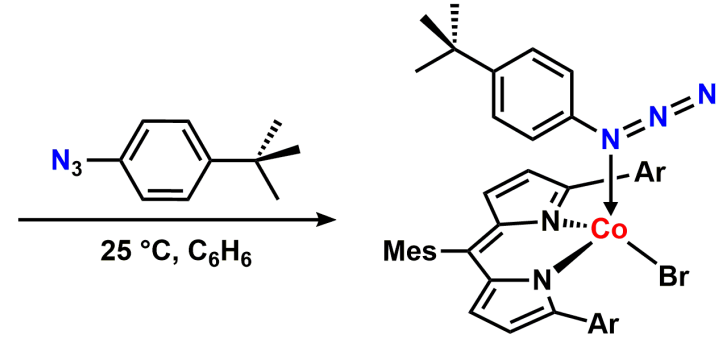

(3)

( $\left.{ }^{\mathrm{Ar}} \mathbf{L}\right) \mathbf{C o B r}\left(\mathbf{N}_{\mathbf{3}}\left(\mathbf{C}_{6} \mathbf{H}_{4-}-{ }^{-}{ }^{t} \mathbf{B u}\right)\right)$, (3): In an oven-dried $20 \mathrm{~mL}$ vial, a solution of $\left({ }^{\mathrm{Ar}} \mathbf{L}\right) \mathbf{C o B r}$ (2) (50 $\mathrm{mg}, 0.05 \mathrm{mmol}, 1.0$ equiv) in benzene was added to a benzene solution of 1-azido-4-(tertbutyl)benzene (1.0 equiv) to observe an immediate color change from purple to reddish purple. The solution was lyophilized to afford 3 as a purple powder $(53 \mathrm{mg}, 90 \%)$. ${ }^{1} \mathbf{H} \mathbf{~ N M R}(600 \mathrm{MHz}$, $\mathrm{C}_{6} \mathrm{D}_{6}$ ): $\delta / \mathrm{ppm} 77.54$ (s), 24.12 (s), 21.05 (s), 14.62 (s), 11.81 (s), 11.20 (s), 10.78 (s), -0.71 (s), -16.64 (s), -28.07 (s). Anal. Calc. for $\mathrm{C}_{76} \mathrm{H}_{62} \mathrm{BrCoN}_{5}$ : C 77.08, H 5.28, N 5.91; Found: C 77.10, $\mathrm{H}$ 5.39, N 5.87. Crystals suitable for X-ray diffraction were grown from a $n$-pentane:toluene solution at $-35^{\circ} \mathrm{C}$.

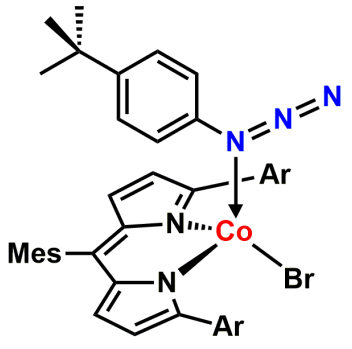

(3)

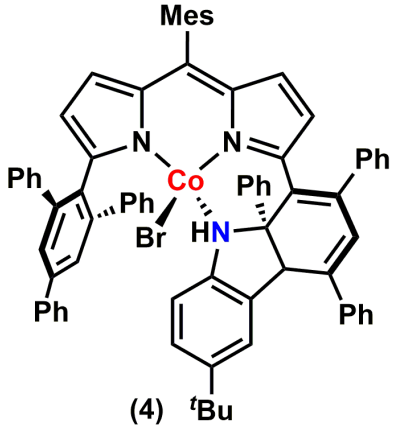

(4) ${ }^{t} \mathrm{Bu}$

( $\left.{ }^{\mathrm{Ar}} \mathbf{L}\right) \operatorname{CoBr}([3+2]$ annulation) (4): A benzene solution of 3 (50 $\mathrm{mg}, 0.042 \mathrm{mmol})$ was heated to 50 ${ }^{\circ} \mathrm{C}$ in a J. Young NMR tube for 20 hours to yield a blue-purple solution. Alternatively, a solution of $\mathbf{3}$ in benzene was stirred at room temperature for 3 days to afford $\mathbf{4}$ quantitatively. The solution was lyophilized to afford 4 as a purple powder (45 mg, 93\%). ${ }^{1} \mathbf{H}$ NMR $\left(600 \mathrm{MHz}, \mathrm{C}_{6} \mathrm{D}_{6}\right): \delta / \mathrm{ppm}$ 68.00 (s), 55.54 (s), 29.68 (s), 26.35 (s), 20.67 (s), 14.11 (s), 13.12 (s), 10.89 (s), 10.18 (s), 9.32 (s), $8.56(\mathrm{~s}), 5.76(\mathrm{~s}), 5.00(\mathrm{~s}), 4.28(\mathrm{~s}),-5.40(\mathrm{~s}),-5.92(\mathrm{~s}),-9.28(\mathrm{~s}),-10.88$ (s). Anal. Calc. for $\mathrm{C}_{76} \mathrm{H}_{62} \mathrm{BrCoN}_{3}$ : C 78.95, H 5.41, N 3.63; Found: C 79.05, H 5.35, N 3.70. Crystals suitable for Xray diffraction were grown from a $n$-hexane:benzene solution at $-35^{\circ} \mathrm{C}$. 


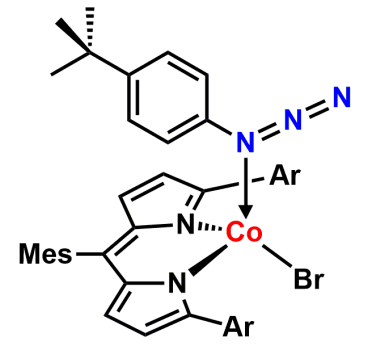

(3)

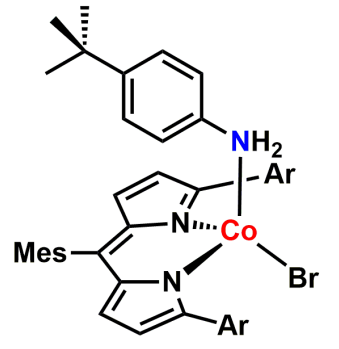

(5)

( $\left.{ }^{\mathrm{Ar}} \mathbf{L}\right) \mathbf{C o B r}\left(\mathbf{N H}_{2}\left(\mathbf{C}_{6} \mathbf{H}_{4-}-{ }^{-}{ }^{t} \mathbf{B u}\right)\right.$ ), (5): In an oven-dried $20 \mathrm{~mL}$ vial, a benzene solution of 3 (50 $\mathrm{mg}$, $0.042 \mathrm{mmol}, 1.0$ equiv) was added to an excess amount of 1,4-cyclohexadiene (5.0 equiv) and the mixture was transferred to a J. Young NMR tube and heated to $50{ }^{\circ} \mathrm{C}$ for 12 hours. The solution was lyophilized to yield 5 as a reddish-purple powder (43 mg, 87\%). ${ }^{1} \mathbf{H}$ NMR $\left(600 \mathrm{MHz}, \mathrm{C}_{6} \mathrm{D}_{6}\right)$ :

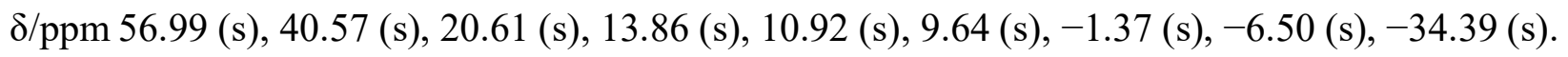
Anal. Calc. for $\mathrm{C}_{76} \mathrm{H}_{64} \mathrm{BrCoN}_{3}$ : C 78.81, H 5.57, N 3.63; Found: C 78.52, H 5.99, N 3.69. Crystals suitable for X-ray diffraction were grown from a $n$-hexane:benzene solution at room temperature.

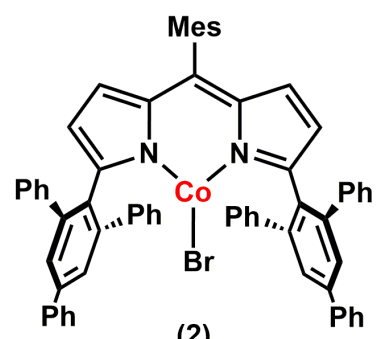

(2)

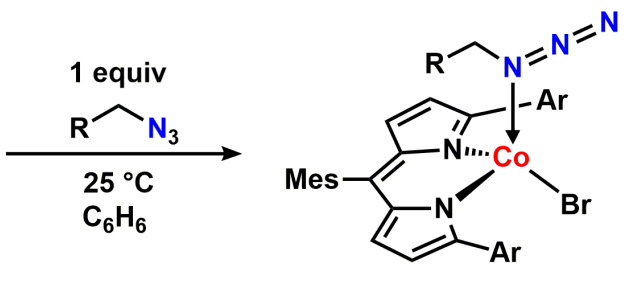

(6-8)

General procedure: In an oven-dried $20 \mathrm{~mL}$ vial, a diluted solution of alkyl azide (1.0 equiv) in benzene was added to a stirring solution of $\left({ }^{\mathrm{Ar}} \mathrm{L}\right) \mathrm{CoBr}(2)(50 \mathrm{mg}, 0.05 \mathrm{mmol} 1.0$ equiv) to observe an immediate color change from purple to reddish purple. The solution was lyophilized to afford the corresponding azide adduct as a purple powder.

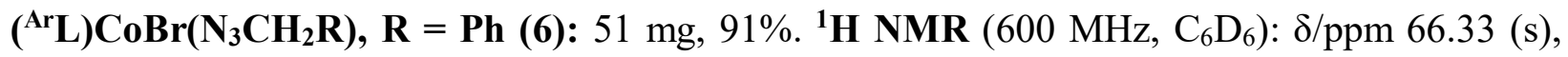
64.75 (s), 16.19 (s), 8.69 (s), -0.38 (s), -2.04 (s). Anal. Calc. for $\mathrm{C}_{73} \mathrm{H}_{56} \mathrm{BrCoN}_{5}$ : C 76.77, H 4.94, N 6.13; Found: C 76.25, H 5.10, N 6.45.

$\left({ }^{\mathrm{Ar}} \mathbf{L}\right) \mathbf{C o B r}\left(\mathbf{N}_{3} \mathbf{C H}_{2} \mathbf{R}\right), \mathbf{R}=\left(\mathbf{C H}_{2}\right)_{\mathbf{3}}\left(\mathbf{C}_{\mathbf{6}} \mathbf{H}_{5}\right)(\mathbf{7}): 53 \mathrm{mg}, 90 \% .{ }^{\mathbf{1}} \mathbf{H} \mathbf{N M R}\left(500 \mathrm{MHz}, \mathrm{C}_{6} \mathrm{D}_{6}\right): \delta / \mathrm{ppm}$ 65.48 (s), 29.40 (s), 17.48 (s), 9.27 (s), 8.79 (s), 6.30 (s), 4.95 (s), -2.00 (s), -24.71 (s). Anal. 
Calc. for $\mathrm{C}_{76} \mathrm{H}_{62} \mathrm{BrCoN}_{5}$ : C 77.08, H 5.28, N 5.91; Found: C 77.00, H 5.35, N 5.60. Crystals suitable for $\mathrm{X}$-ray diffraction were grown from a $n$-hexane:benzene solution at room temperature. $\left({ }^{\mathrm{Ar}} \mathbf{L}\right) \mathbf{C o B r}\left(\mathbf{N}_{3} \mathbf{C H}_{2} \mathbf{R}\right), \mathbf{R}=\left(\mathbf{C H}_{2}\right)_{2} \mathbf{C H}\left(\mathbf{C H}_{3}\right)_{2} \mathbf{( 8 )}: 53 \mathrm{mg}, 95 \% .{ }^{1} \mathbf{H} \mathbf{N M R}\left(600 \mathrm{MHz}, \mathrm{C}_{6} \mathrm{D}_{6}\right): \delta / \mathrm{ppm}$ $65.01(\mathrm{~s}), 62.36(\mathrm{~s}), 30.92(\mathrm{~s}), 16.85(\mathrm{~s}), 8.62(\mathrm{~s}), 8.29(\mathrm{~s}), 2.24(\mathrm{~s}),-1.85(\mathrm{~s}),-22.72(\mathrm{~s})$. Anal. Calc. for $\mathrm{C}_{72} \mathrm{H}_{62} \mathrm{BrCoN}_{5}$ : C 76.12, H 5.50, N 6.16; Found: C 75.80, H 5.19, N 5.46. Crystals suitable for X-ray diffraction were grown from a $n$-hexane:benzene solution at room temperature.

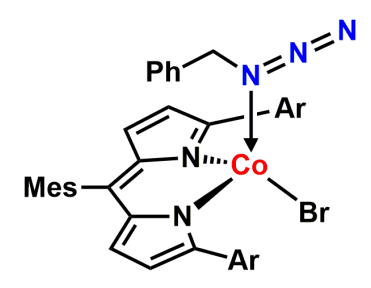

(6)

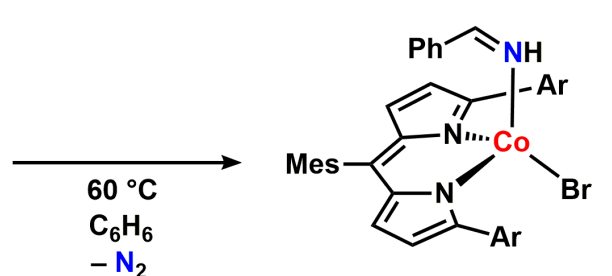

(9)

$\left({ }^{\mathrm{Ar}} \mathbf{L}\right) \mathbf{C o B r}\left(\mathrm{NHCHC}_{6} \mathbf{H}_{5}\right)$ (9): A benzene solution of $6(50 \mathrm{mg}, 0.044 \mathrm{mmol})$ was heated to $60{ }^{\circ} \mathrm{C}$ for 24 hours in a J. Young NMR tube. The solution was lyophilized to afford 9 as a purple powder (42 mg, 87\%). ${ }^{1} \mathbf{H}$ NMR (600 MHz, $\mathrm{C}_{6} \mathrm{D}_{6}$ ): 8/ppm 55.98 (s), 33.15 (s), 11.19 (s), 10.55 (s), 4.72 (s), 4.53 (s), 0.65 (s), -2.94 (s). Anal. Calc. for $\mathrm{C}_{73} \mathrm{H}_{56} \mathrm{BrCoN}_{3}$ : C 78.70, H 5.07, N 3.77; Found: $\mathrm{C} 79.05, \mathrm{H} 4.89, \mathrm{~N}$ 4.02. Crystals suitable for X-ray diffraction were grown from a $n$ pentane:toluene solution at room temperature. 


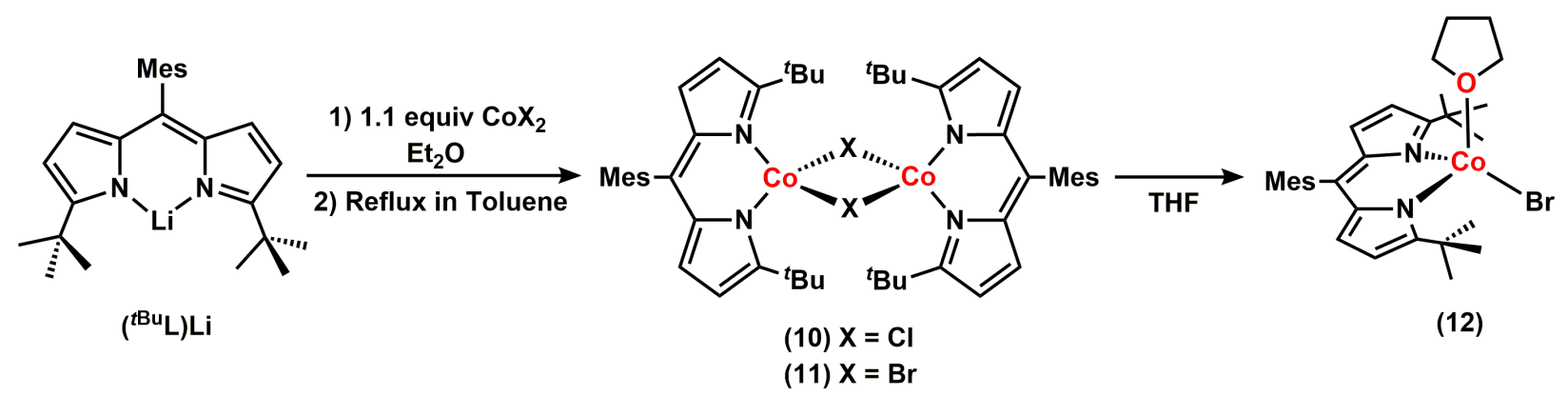

General procedure: In an oven-dried $20 \mathrm{~mL}$ vial, anhydrous $\mathrm{CoX}_{2}(\mathrm{X}=\mathrm{Cl}$ or $\mathrm{Br}, 1.1$ equiv) was dissolved in $3 \mathrm{~mL}$ of diethyl ether. The vial was placed in a liquid nitrogen-cooled cold well until frozen. In a separate vial, $\left({ }^{t \mathrm{Bu}} \mathrm{L}\right) \mathrm{Li}(0.2 \mathrm{~g}, 0.53 \mathrm{mmol}, 1.0$ equiv) was dissolved in $6 \mathrm{~mL}$ of diethyl ether and added to the thawing slurry of $\mathrm{CoX}_{2}$. The reaction mixture was stirred for 4 hours at room temperature. The dark brown mixture was filtered through a coarse glass frit with Celite to remove lithium salts and excess cobalt halide, followed by washing with diethyl ether. The filtrate was concentrated in vacuo, redissolved in benzene, and filtered through a medium porosity glass frit with Celite. The Celite was washed with an additional $20 \mathrm{~mL}$ benzene and the filtrate was lyophilized. The dried powder was refluxed in toluene for $30 \mathrm{~min}$ to yield a bright red solution. The solvent was then removed in vacuo while heating. The dried material was dissolved in benzene and the solution was lyophilized to yield the corresponding dimeric species as a red powder.

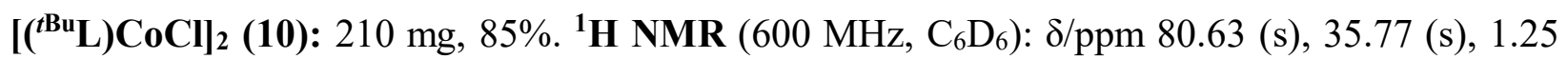
(s), -15.60 (s). Anal. Calc. for $\mathrm{C}_{52} \mathrm{H}_{66} \mathrm{Cl}_{2} \mathrm{Co}_{2} \mathrm{~N}_{4}$ : C 66.74, H 7.11, N 5.99; Found: C 66.95, H 6.75, N 5.85 .

$\left[\left({ }^{\left({ }^{\mathrm{Bu}} \mathbf{L}\right) \mathbf{C o B r}}\right]_{2}\right.$, (11): $245 \mathrm{mg}, 91 \% .{ }^{1} \mathbf{H}$ NMR $\left(600 \mathrm{MHz}, \mathrm{C}_{6} \mathrm{D}_{6}\right): \delta / \mathrm{ppm} 79.85$ (s), 33.09 (s), -13.93. Anal. Calc. for $\mathrm{C}_{52} \mathrm{H}_{66} \mathrm{Br}_{2} \mathrm{Co}_{2} \mathrm{~N}_{4}$ : C 60.95, H 6.49, N 5.47; Found: C 70.10, H 6.90, N 5.60. Crystals suitable for X-ray diffraction were grown from a $n$-hexane/benzene mixture at room temperature.

${ }^{\left({ }^{(B u} \mathbf{L}\right) C o B r(t h f), ~(12): ~ I n ~ a n ~ o v e n-d r i e d ~} 20 \mathrm{~mL}$ vial, $11(20 \mathrm{mg}, 0.02 \mathrm{mmol})$ was dissolved in 3 $\mathrm{mL}$ of THF to afford a dark brown solution. The THF was removed in vacuo and the dried material was redissolved in benzene and lyophilized to yield $\mathbf{1 2}$ as a green powder (22 mg, 97\%). ${ }^{1} \mathbf{H}$ NMR (600 MHz, $\mathrm{C}_{6} \mathrm{D}_{6}$ ): $\delta / \mathrm{ppm} 64.17$ (s), 41.27 (s), 11.08 (s), 9.40 (s), 4.93 (s), 3.52 (s), 2.19 (s), 1.35 (s). Anal. Calc. for $\mathrm{C}_{30} \mathrm{H}_{41} \mathrm{BrCoN}_{2} \mathrm{O}$ : C 61.65, H 7.07, N 4.79; Found: C 61.30, H 6.95, N 4.55. 
Crystals suitable for X-ray diffraction were grown from a $n$-hexane/THF mixture at room temperature.

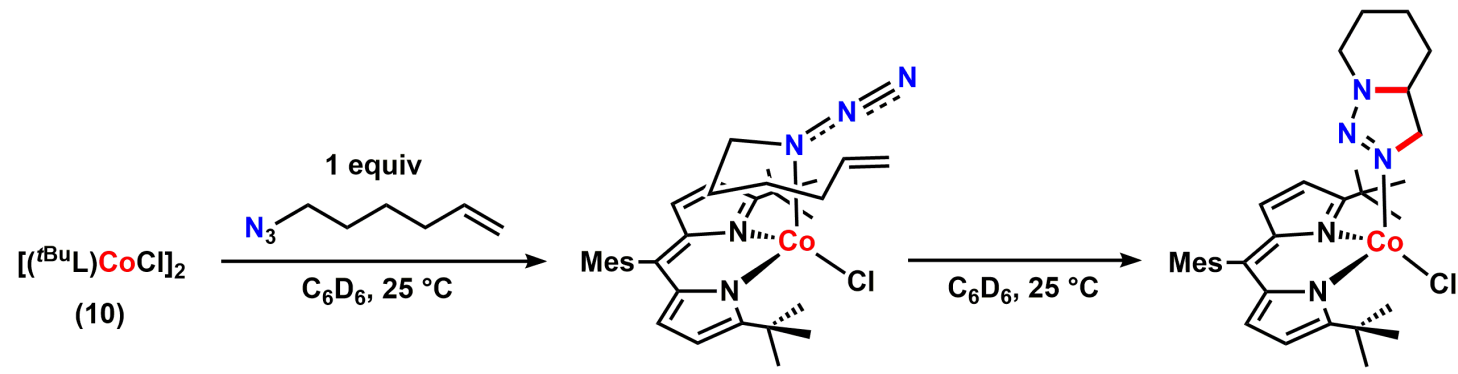

(13)

(14)

$\left({ }^{t B u} \mathbf{L}\right) \mathbf{C o X}\left(\mathbf{N}_{3} \mathbf{R}\right), \mathbf{R}=\left(\mathbf{C H}_{2}\right)_{4}\left(\mathbf{C}_{2} \mathbf{H}_{3}\right):$ In an oven-dried $20 \mathrm{~mL}$ vial, a benzene solution of 6azidohex-1-ene (1.0 equiv per Co center) was added to a stirring solution of $\mathbf{1 0}$ or $\mathbf{1 1}$ (0.02 mmol) in benzene at room temperature to observe an immediate color change from red to brown. Owing to the rapid conversion to the corresponding triazole bound species, the azide bound complex $\mathbf{1 3}$ could not be isolated. $\mathbf{X}=\mathbf{B r}:{ }^{1} \mathbf{H}$ NMR (600 MHz, $\left.\mathrm{C}_{6} \mathrm{D}_{6}\right): \delta / \mathrm{ppm} 70.40$ (s), 41.74 (s), 18.86 (s), $10.78(\mathrm{~s}), 4.34(\mathrm{~s}), 1.37(\mathrm{~s}),-0.17(\mathrm{~s}),-3.07(\mathrm{~s}),-5.14(\mathrm{~s}),-30.57(\mathrm{~s})$.

$\left({ }^{\mathrm{Bu}} \mathrm{L}\right) \mathrm{CoCl}(\mathbf{1 , 2 , 3 - d i h y d r o t r i a z o l e )}$ (14): A benzene solution of 13 (prepared in situ following the procedure described above using $\mathbf{1 0}(20 \mathrm{mg})$ ) was stirred at room temperature for 1 hour. The solution was lyophilized to afford 14 as a brown powder $(17 \mathrm{mg}, 87 \%) .{ }^{1} \mathbf{H}$ NMR (600 MHz, $\mathrm{C}_{6} \mathrm{D}_{6}$ ): $\delta / \mathrm{ppm} 61.44$ (s), 32.52 (s), 27.04 (s), 19.98 (s), 9.30 (s), 3.79 (s), 1.34 (s), -2.83 (s). Anal. Calc. for $\mathrm{C}_{32} \mathrm{H}_{44} \mathrm{ClCoN}_{5}$ : C 64.80, H 7.48, N 11.81; Found: C 65.05, H 7.75, N 12.02.

$\left({ }^{\mathrm{Bu}} \mathrm{L}\right) \mathrm{CoBr}(1,2,3-d i h y d r o t r i a z o l e) ~(14-B r): 24 \mathrm{mg}, 95 \% .{ }^{1} \mathbf{H}$ NMR $\left(600 \mathrm{MHz}, \mathrm{C}_{6} \mathrm{D}_{6}\right): \delta / \mathrm{ppm}$ 62.91 (s), 60.45 (s), 60.28 (s), 31.00 (s), 28.95 (s), 24.86 (s), 9.73 (s), 9.00 (s), 5.92 (s), 3.84 (s), 1.34 (s), -3.69 (s). Anal. Calc. for $\mathrm{C}_{32} \mathrm{H}_{44} \mathrm{BrCoN}_{5}$ : C 60.28, H 6.96, N 10.98; Found: C 60.45, H 7.05, N 11.10. Crystals suitable for X-ray diffraction were grown from a $n$-hexane:benzene solution at room temperature. 


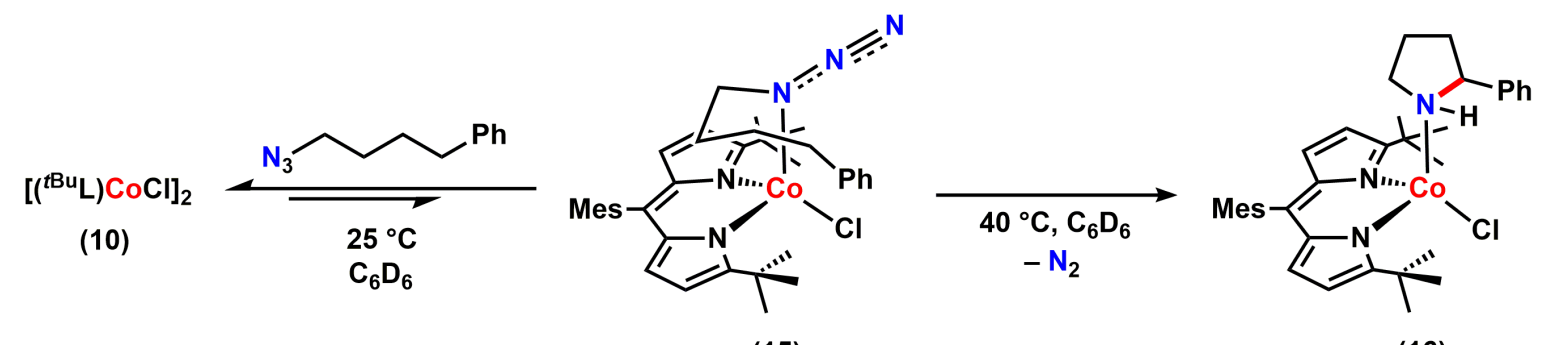

(15)

(16)

$\left({ }^{t{ }^{B u}} \mathbf{L}\right) \mathbf{C o X}\left(\mathbf{N}_{3} \mathbf{R}\right), \mathbf{R}=\left(\mathbf{C H}_{2}\right)_{4}\left(\mathbf{C}_{6} \mathbf{H}_{5}\right)$ : In an oven-dried $20 \mathrm{~mL}$ vial, a benzene solution of (4azidobutyl)benzene (1.0 equiv per Co center) was added to a stirring solution of $\mathbf{1 0}$ or $\mathbf{1 1}(0.02$ $\mathrm{mmol}$ ) in benzene at room temperature to observe an immediate color change from red to brown. Owing to a greater driving force to regenerate the dimeric species (10 or $\mathbf{1 1})$ during crystallization, the azide adduct complex was not isolable in the solid state. $\mathbf{X}=\mathbf{C l}$ (15): ${ }^{1} \mathbf{H}$ NMR (600 MHz, $\mathrm{C}_{6} \mathrm{D}_{6}$ ): $\delta / p p m 66.39$ (s), 47.19 (s), 8.92 (s), 8.30 (s), 6.95 (s), 6.50 (s), 4.48 (s), 2.14 (s), 1.40 (s), 1.31 (s), -0.88 (s), -0.54 (s), -1.70 (s), -15.98 (s), -32.26 (s). X = Br: ${ }^{1}$ H NMR (600 MHz, $\left.\mathrm{C}_{6} \mathrm{D}_{6}\right)$ :

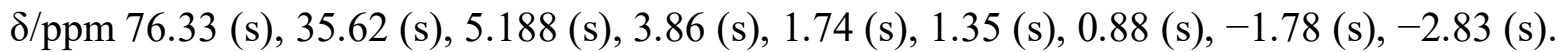

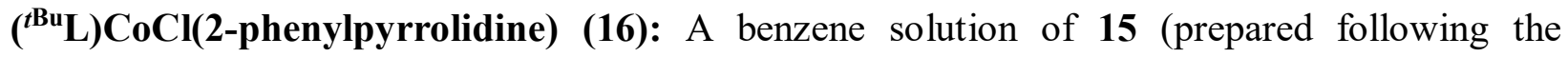
procedure described above using $10(20 \mathrm{mg})$ ) was heated to $40^{\circ} \mathrm{C}$ for 24 hours in a J. Young NMR tube. The solution was lyophilized to afford 16 as a brown powder (23 $\mathrm{mg}, 89 \%)$. ${ }^{\mathbf{1}} \mathbf{H}$ NMR (600 $\mathrm{MHz}, \mathrm{C}_{6} \mathrm{D}_{6}$ ): $\delta / \mathrm{ppm} 62.91$ (s), 60.45 (s), 60.28 (s), 31.00 (s), 28.95 (s), 24.86 (s), 9.73 (s), 9.00 (s), $5.92(\mathrm{~s}), 3.84(\mathrm{~s}), 1.34(\mathrm{~s}),-3.69(\mathrm{~s})$.

( $\left.{ }^{t B u} \mathrm{~L}\right)$ CoBr(2-phenylpyrrolidine) (16-Br): $21 \mathrm{mg}, 84 \% .{ }^{1} \mathbf{H}$ NMR $\left(600 \mathrm{MHz}, \mathrm{C}_{6} \mathrm{D}_{6}\right): \delta / \mathrm{ppm}$ 62.91 (s), 60.45 (s), 60.28 (s), 31.00 (s), 28.95 (s), 24.86 (s), 9.73 (s), 9.00 (s), 5.92 (s), 3.84 (s), 1.34 (s), -3.69 (s). Anal. Calc. for $\mathrm{C}_{36} \mathrm{H}_{46} \mathrm{BrCoN}_{3}$ : C 65.55, H 7.03, N 6.37; Found: C 65.27, H 7.22, N 5.98. Crystals suitable for X-ray diffraction were grown from a $n$-hexane:benzene solution at room temperature. 


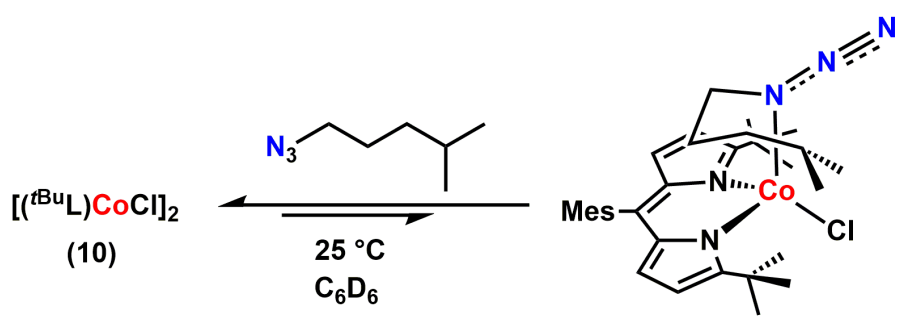

(17)

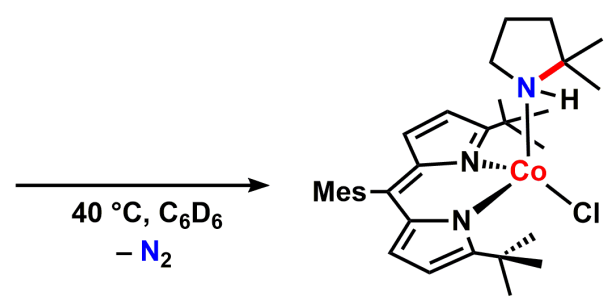

(18)

$\left({ }^{\mathrm{Bu}} \mathbf{L}\right) \mathbf{C o X}\left(\mathrm{N}_{3} \mathrm{R}\right), \mathbf{R}=\left(\mathrm{CH}_{2}\right)_{3} \mathbf{C H}\left(\mathrm{CH}_{3}\right)_{2}$ (17): In an oven-dried $20 \mathrm{~mL}$ vial, a benzene solution of 1-azido-4-methylpentane (1.0 equiv per Co center) was added to a stirring solution of $\mathbf{1 0}$ or $\mathbf{1 1}$ $(0.02 \mathrm{mmol})$ in benzene at room temperature to observe an immediate color change from red to brown. Owing to a greater driving force to regenerate the dimeric species (10 or 11) during crystallization, the azide-bound complex was not isolable in the solid state. $\mathbf{X}=\mathbf{C l}$ (17): ${ }^{1} \mathbf{H}$ NMR (600 MHz, $\mathrm{C}_{6} \mathrm{D}_{6}$ ): $\delta / \mathrm{ppm} 67.00$ (s), 46.95 (s), 9.68 (s), 8.28 (s), 4.68 (s), 2.14 (s), 1.31 (s), -0.11 (s), -1.21 (s), -1.91 (s), -32.15 (s). $\mathbf{X}=\mathbf{B r}:{ }^{1} \mathbf{H}$ NMR (600 MHz, $\left.\mathrm{C}_{6} \mathrm{D}_{6}\right)$ : $\delta / \mathrm{ppm} 75.68$ (s), 36.26 (s), $9.96(\mathrm{~s}), 3.86(\mathrm{~s}), 1.74(\mathrm{~s}), 1.35(\mathrm{~s}), 0.89(\mathrm{~s}),-0.45(\mathrm{~s}),-2.66(\mathrm{~s}),-4.11(\mathrm{~s}),-11.14(\mathrm{~s})$.

( $\left.{ }^{\mathrm{Ar}} \mathrm{L}\right) \mathrm{CoCl}(\mathbf{2 , 2}$-dimethylpyrrolidine) (18): A benzene solution of 17 (prepared following the procedure described above using $10(20 \mathrm{mg})$ ) was heated to $40{ }^{\circ} \mathrm{C}$ for 24 hours in a J. Young NMR tube. The solution was lyophilized to afford 18 as a brown powder (21 mg, 86\%). ${ }^{1} \mathbf{H}$ NMR (600 MHz, $\left.\mathrm{C}_{6} \mathrm{D}_{6}\right)$ : $\delta / \mathrm{ppm} 62.91$ (s), 60.45 (s), 60.28 (s), 31.00 (s), 28.95 (s), 24.86 (s), 9.73 (s), 9.00 (s), 5.92 (s), 3.84 (s), 1.34 (s), -3.69 (s). Anal. Calc. for $\mathrm{C}_{34} \mathrm{H}_{46} \mathrm{ClCoN}_{3}$ : C 67.77, H 8.18, N 7.41; Found: C 67.53, H 8.35, N 7.78. Crystals suitable for X-ray diffraction were grown from a $n$ hexane:benzene solution at room temperature.

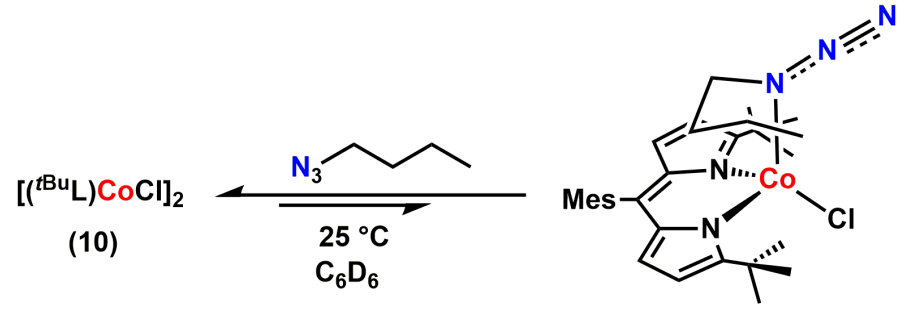

(19)

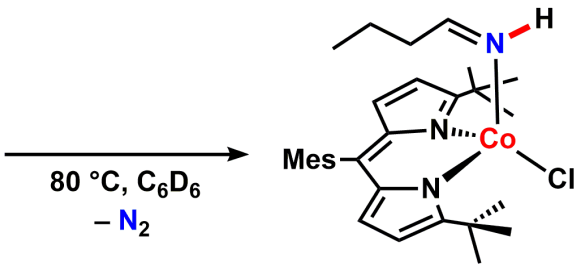

(20)

$\left({ }^{\mathrm{Bu}} \mathbf{L}\right) \mathbf{C o X}\left(\mathrm{N}_{3} \mathbf{R}\right), \mathbf{R}=\left(\mathrm{CH}_{2}\right)_{3} \mathbf{C H}_{3}$ (19): In an oven-dried $20 \mathrm{~mL}$ vial, a benzene solution of 1azidobutane (1.0 equiv per Co center) was added to a stirring solution of 10 or $11(0.02 \mathrm{mmol})$ in benzene at room temperature to observe an immediate color change from red to brown. Owing to 
a greater driving force to regenerate the dimeric species (10 or $\mathbf{1 1})$ during crystallization, the azidebound complex was not isolable in the solid state. $\mathbf{X}=\mathbf{C l}(\mathbf{1 9}):{ }^{1} \mathbf{H} \mathbf{~ N M R}\left(600 \mathrm{MHz}, \mathrm{C}_{6} \mathrm{D}_{6}\right)$ : $\delta / \mathrm{ppm}$ $66.87(\mathrm{~s}), 46.77$ (s), $10.93(\mathrm{~s}), 8.94(\mathrm{~s}), 4.60(\mathrm{~s}), 2.14(\mathrm{~s}), 1.31(\mathrm{~s}),-0.19$ (s), $-0.76(\mathrm{~s}),-1.66$ (s), -31.94 (s). $\mathbf{X}=\mathbf{B r}:{ }^{1} \mathbf{H}$ NMR $\left(600 \mathrm{MHz}, \mathrm{C}_{6} \mathrm{D}_{6}\right)$ : $\delta / \mathrm{ppm} 76.10$ (s), 35.62 (s), 9.58 (s), 7.86 (s), 5.18 (s), $3.77(\mathrm{~s}), 1.71(\mathrm{~s}),-0.74(\mathrm{~s}),-2.04(\mathrm{~s}),-3.71(\mathrm{~s}),-11.13(\mathrm{~s})$.

$\left({ }^{\mathrm{Bu}} \mathrm{L}\right) \mathrm{CoCl}(\mathrm{NHR}), \mathbf{R}=(\mathrm{CH})\left(\mathrm{CH}_{2}\right)_{2} \mathrm{CH}_{3}$ (20): A benzene solution of 19 (prepared following the procedure described above using $10(20 \mathrm{mg})$ ) was heated to $80^{\circ} \mathrm{C}$ for 24 hours in a J. Young NMR tube. The solution was lyophilized to afford 20 as a brown powder (21 mg, 91\%). ${ }^{1} \mathbf{H}$ NMR (600 MHz, $\mathrm{C}_{6} \mathrm{D}_{6}$ ): $\delta / \mathrm{ppm} 190.00$ (s), 152.71 (s), 61.69 (s), 60.60 (s), 31.52 (s), 30.13 (s), 27.05 (s), 16.66 (s), 3.88 (s), 1.37 (s), -2.38 (s), -9.58 (s). Anal. Calc. for $\mathrm{C}_{30} \mathrm{H}_{42} \mathrm{ClCoN}_{3}$ : C 66.84, H 7.85, N 7.80; Found: C 66.50, H 9.01, N 8.15.

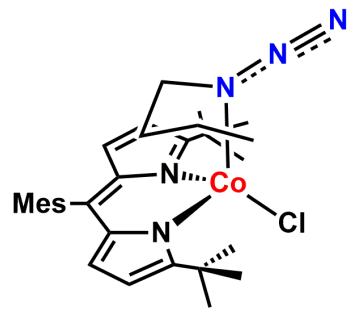

(19)

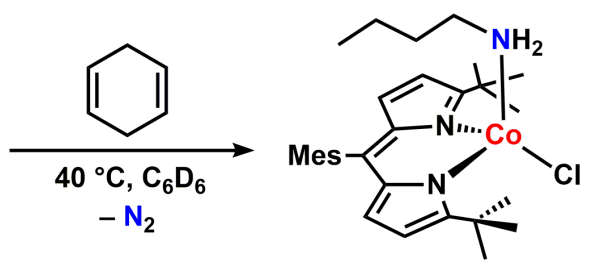

(21)

$\left({ }^{(\mathrm{Bu}} \mathrm{L}\right) \mathrm{CoCl}\left(\mathrm{NH}_{2} \mathrm{R}\right), \mathbf{R}=\left(\mathrm{CH}_{2}\right)_{3} \mathrm{CH}_{3}$ (21): In an oven-dried $20 \mathrm{~mL}$ vial, a benzene solution of 1azidobutane (1.0 equiv per Co center) was added to a stirring solution of $\mathbf{1 0}(20 \mathrm{mg})$. To the stirring mixture, excess amount of 1,4-cyclohexadiene (10 equiv) was added. The mixture was transferred to a J. Young NMR tube and heated to $40^{\circ} \mathrm{C}$ for 24 hours to observe a new paramagnetic species (21) by ${ }^{1} \mathrm{H}$ NMR spectroscopy. The authentic $\mathbf{2 1}$ can be prepared from $\mathbf{1 0}$ and a stoichiometric amount of butan-1-amine in benzene at room temperature. The dark brown mixture was lyophilized to afford $\mathbf{2 1}$ as a brown powder in quantitative yield (Figure S-11). ${ }^{\mathbf{1}} \mathbf{H}$ NMR (600 MHz, $\mathrm{C}_{6} \mathrm{D}_{6}$ ): $\delta / \mathrm{ppm} 61.83$ (s), 31.67 (s), 29.77 (s), 8.96 (s), 5.65 (s), 3.59 (s), 0.39 (s), -0.30 (s). Anal. Calc. for $\mathrm{C}_{30} \mathrm{H}_{44} \mathrm{ClCoN}_{3}$ : C 66.59, H 8.20, N 7.77; Found: C 66.90, H 7.92, N 7.45. Crystals suitable for X-ray diffraction were grown from a $n$-hexane:benzene solution at room temperature. 


\section{Non-Covalent Interaction Analysis. ${ }^{8-9}$}

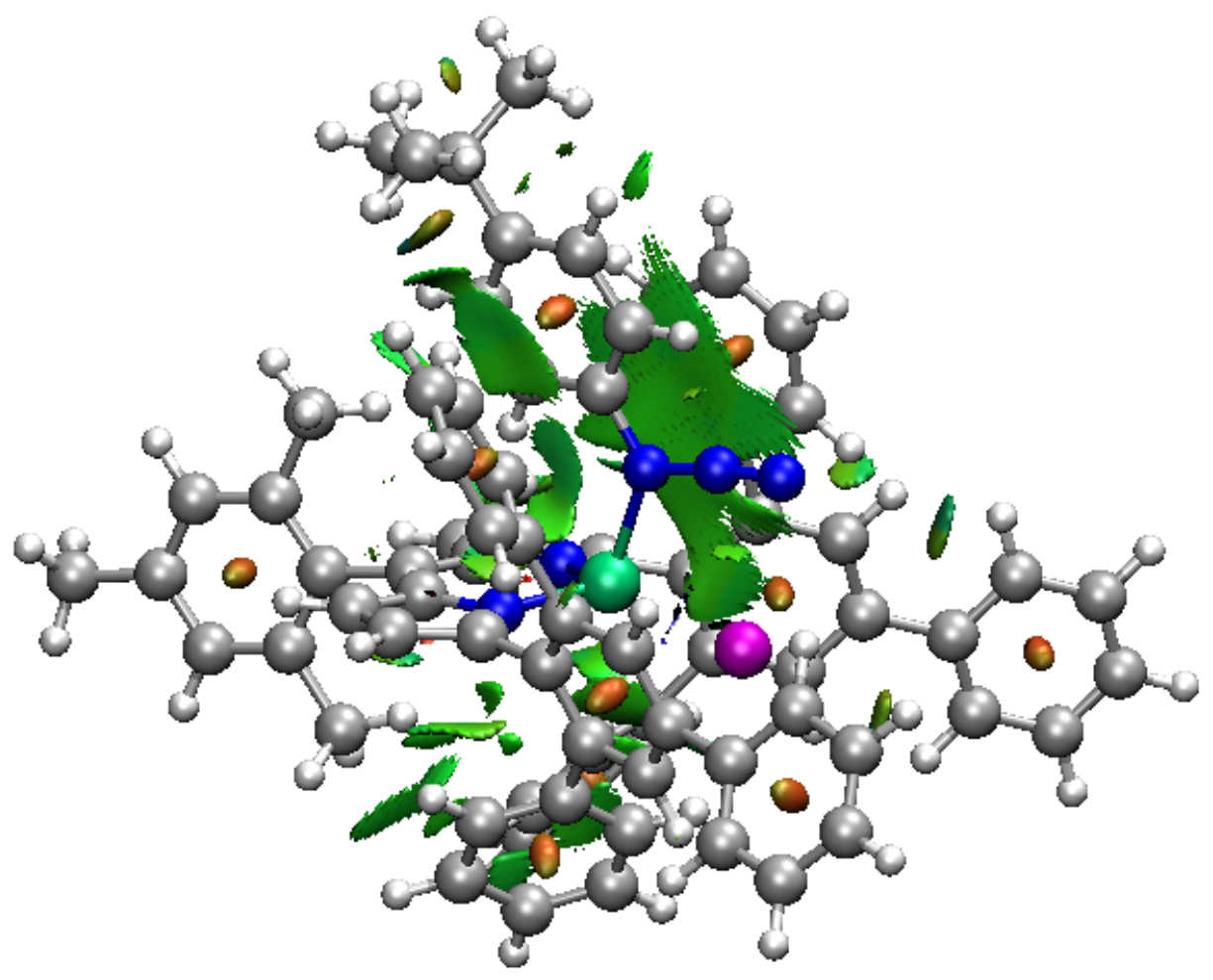

Figure S-1. Non-covalent interaction plot for 3 . 


\section{Stoichiometric Reactions.}

\section{Reaction with cyclohexene.}

To a stirring solution of $2(20 \mathrm{mg}, 0.02 \mathrm{mmol})$ in $0.7 \mathrm{~mL}$ of cyclohexene, a solution of 1-azido-4(tert-butyl)benzene (1.0 equiv, $0.02 \mathrm{mmol}$ ) diluted in $0.3 \mathrm{~mL}$ of cyclohexene was added and allowed to stir at room temperature for 24 hours under an inert $\mathrm{N}_{2}$ atmosphere. The reaction was removed from the glove box, concentrated in vacuo, and ran through a neutral alumina gel eluting with a 10:1 mixture of dichloromethane and methanol to remove paramagnetic materials. ${ }^{1} \mathrm{H}$ NMR and GC-MS revealed formation of 4-(tert-butyl)- $N$-(cyclohex-2-en-1-yl)aniline. ${ }^{10}$ The yield was determined via ${ }^{1} \mathrm{H}$ NMR integration using 1,3,5-trimethoxybezene as an internal standard.
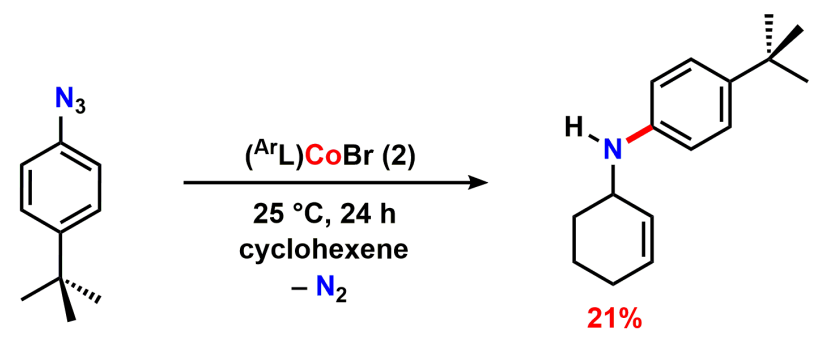


\section{Reaction with styrene.}

To a stirring solution of $2(20 \mathrm{mg}, 0.02 \mathrm{mmol})$ in benzene, a solution of 1-azido-4-(tertbutyl)benzene ( 1.0 equiv, $0.02 \mathrm{mmol}$ ) diluted in benzene was added followed by styrene (200 equiv, $4 \mathrm{mmol}$ ). The reaction mixture was allowed to stir at room temperature for 24 hours. The reaction was removed from the glove box, concentrated in vacuo, and ran through a neutral alumina gel eluting with a 10:1 mixture of dichloromethane and methanol to remove paramagnetic materials. ${ }^{1} \mathrm{H} \quad \mathrm{NMR}$ and GC-MS revealed formation of 1-(4-(tert-butyl)phenyl)-2phenylaziridine. ${ }^{11}$ The yield was determined via ${ }^{1} \mathrm{H}$ NMR integration using 1,3,5trimethoxybezene as an internal standard.
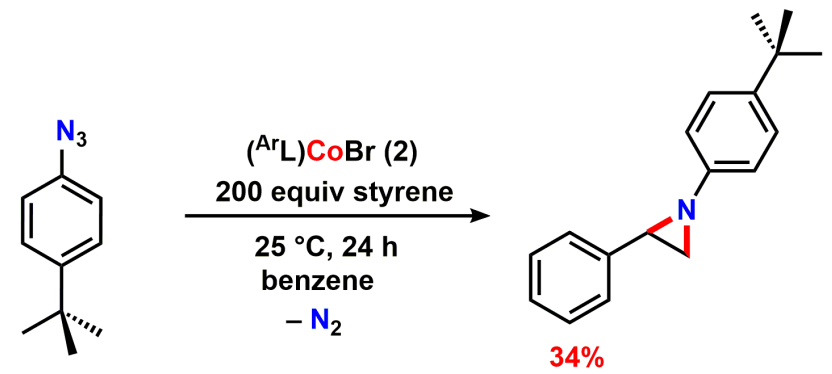


\section{Spectroscopic Characterization.}

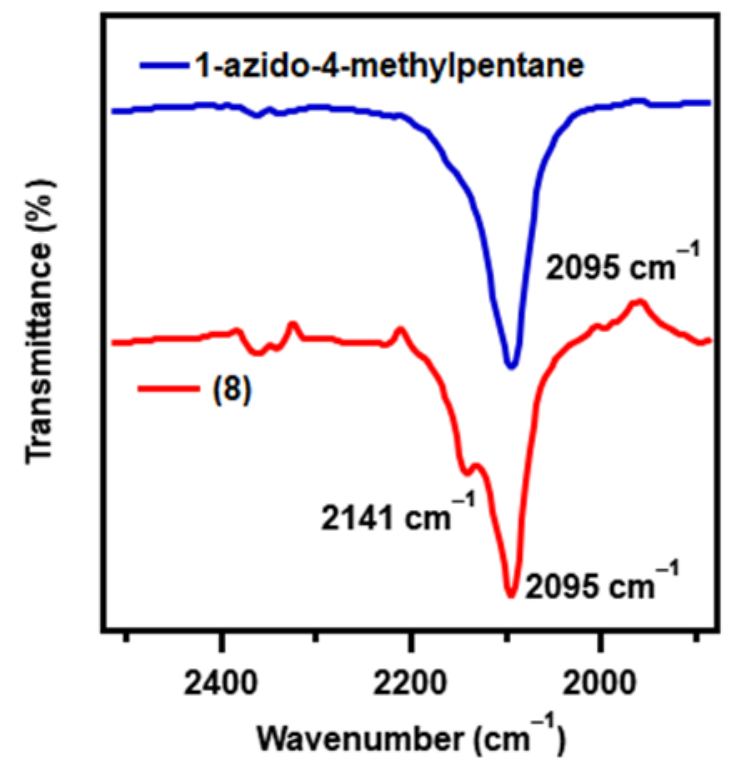

Figure S-2. Solution IR spectra of 1-azido-4-methylpentane and $\mathbf{8}$ in benzene in the presence of a slight excess amount of 1-azido-4-methylpentane.

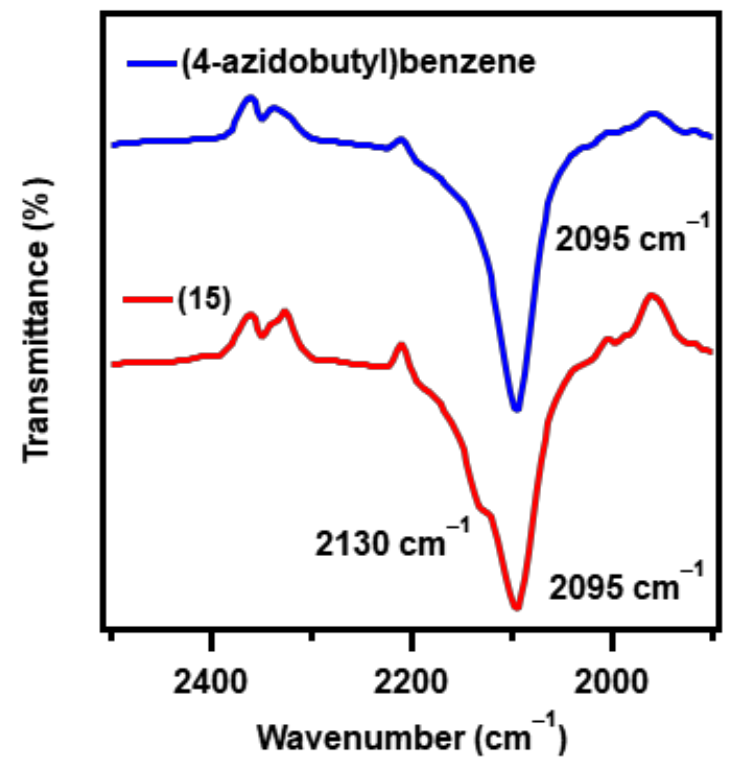

Figure S-3. Solution IR spectra of (4-azidobutyl)benzene and $\mathbf{1 5}$ in benzene in the presence of a slight excess amount of (4-azidobutyl)benzene. 


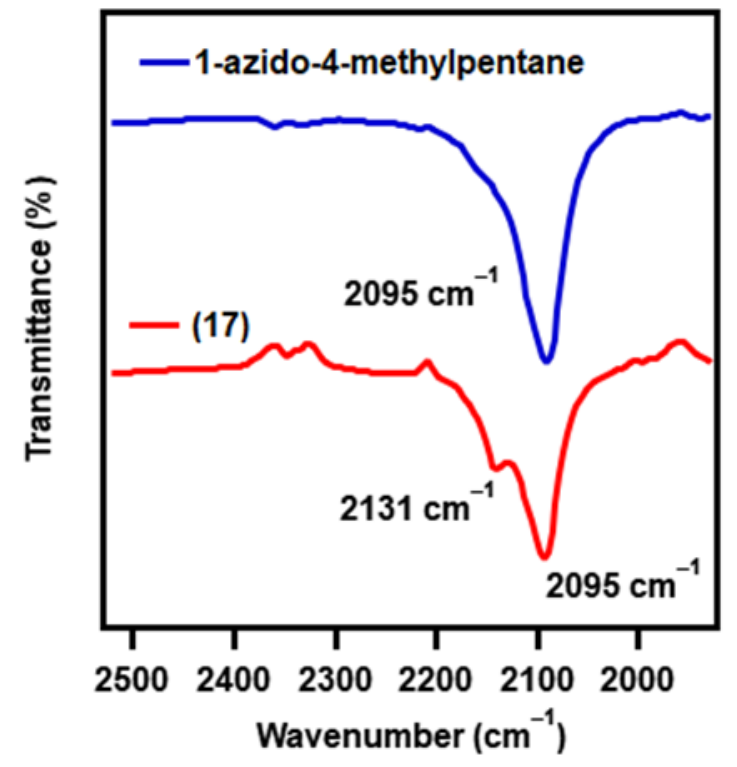

Figure S-4. Solution IR spectra of 1-azido-4-methylpentane and 17 in benzene in the presence of a slight excess amount of 1-azido-4-methylpentane.

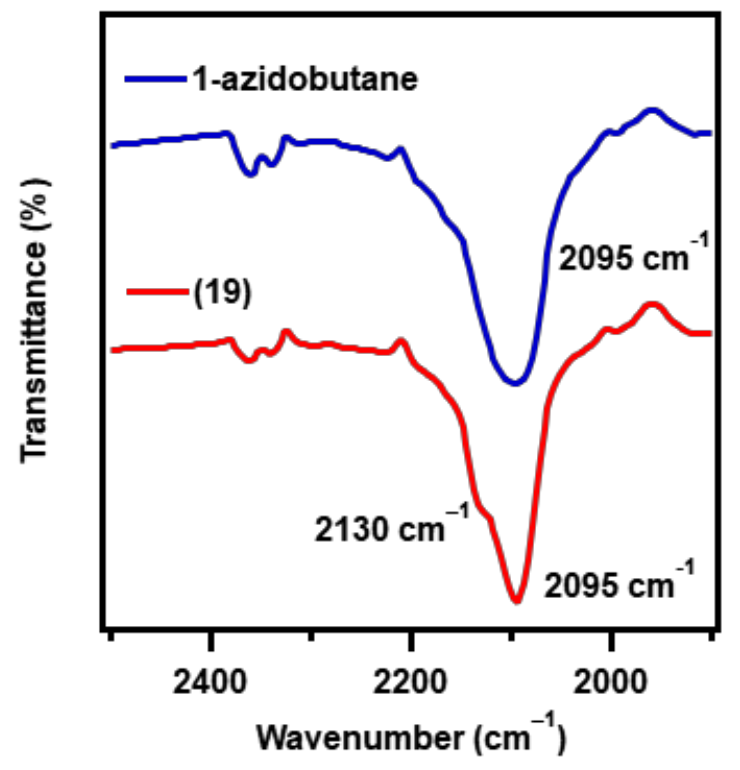

Figure S-5. Solution IR spectra of 1-azidobutane and 19 in benzene in the presence of a slight excess amount of 1-azidobutane. 

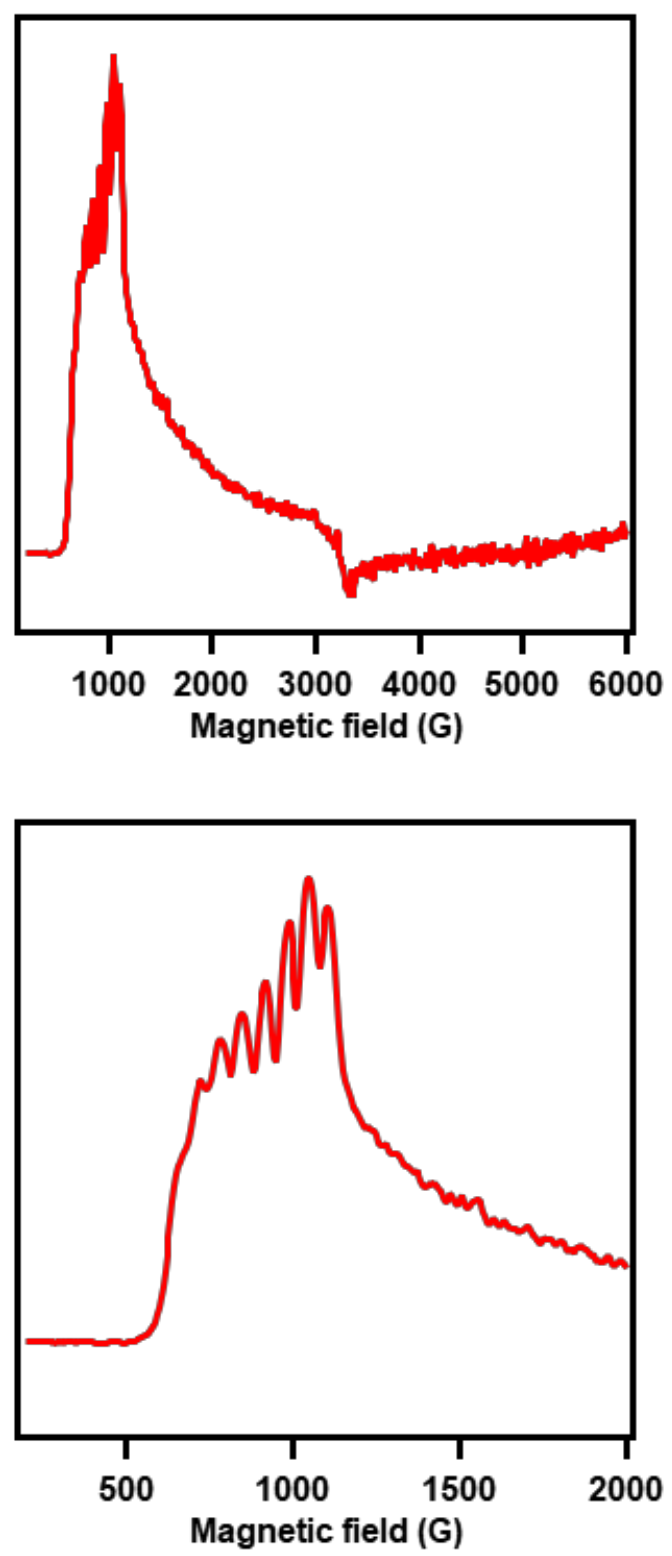

Figure S-6. Frozen toluene EPR spectrum of 8 at $4 \mathrm{~K}$. 


\section{Reactivity Studies on 8.}

$\left({ }^{\mathrm{Ar}} \mathrm{L}\right) \mathrm{CoBr}\left(\mathrm{N}_{3} \mathrm{R}\right)(\mathbf{8})(15 \mathrm{mg}, 0.013 \mathrm{mmol})$ was dissolved in $0.7 \mathrm{~mL}$ of benzene- $d_{6}$ and the solution was transferred to a J. Young NMR tube. The reaction mixture was heated to $60{ }^{\circ} \mathrm{C}$ and its progression was monitored by ${ }^{1} \mathrm{H}$ NMR spectroscopy. ${ }^{1} \mathrm{H}$ NMR chemical shifts corresponding to the proposed nitrene intermediate are colored in red and the ones for the final imine adduct are colored in blue. The final imine adduct can be crystallized at room temperature from $n$ hexane/benzene mixture, however, the obtained X-ray structure cannot be modeled owing to the intrinsic disorder of the whole molecule. Instead, upon quenching the crude reaction mixture, the bound imine readily hydrolyzes to the corresponding 4-methylpentanal as confirmed by ${ }^{1} \mathrm{H}$ NMR spectroscopy supporting the identity of the bound imine moiety. ${ }^{12}$

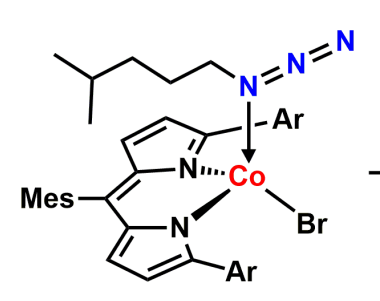

(8)

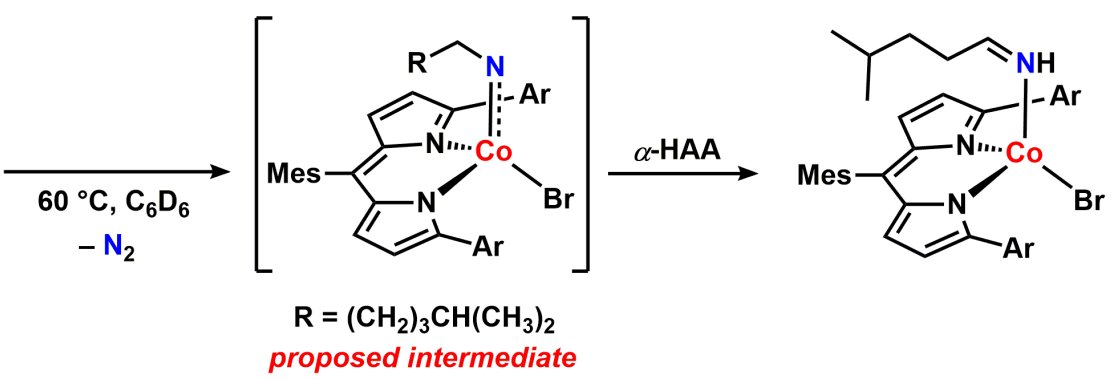

(a) Complex (8)

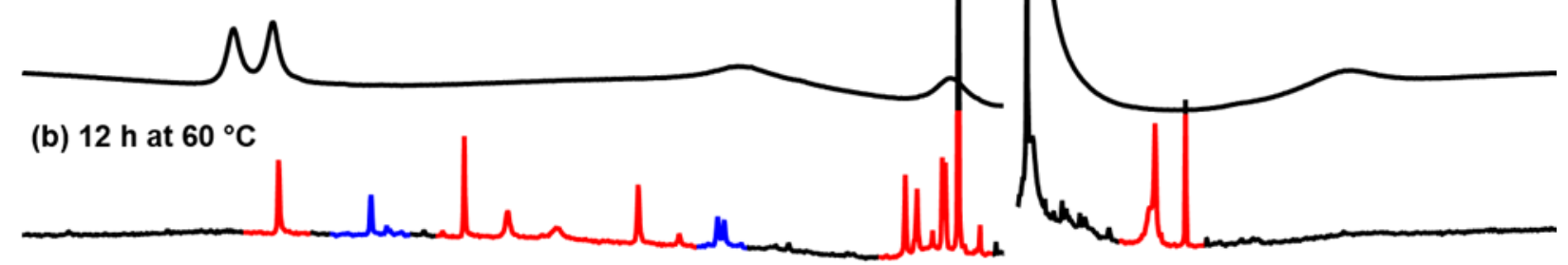

(c) $24 \mathrm{~h}$ at $60^{\circ} \mathrm{C}$

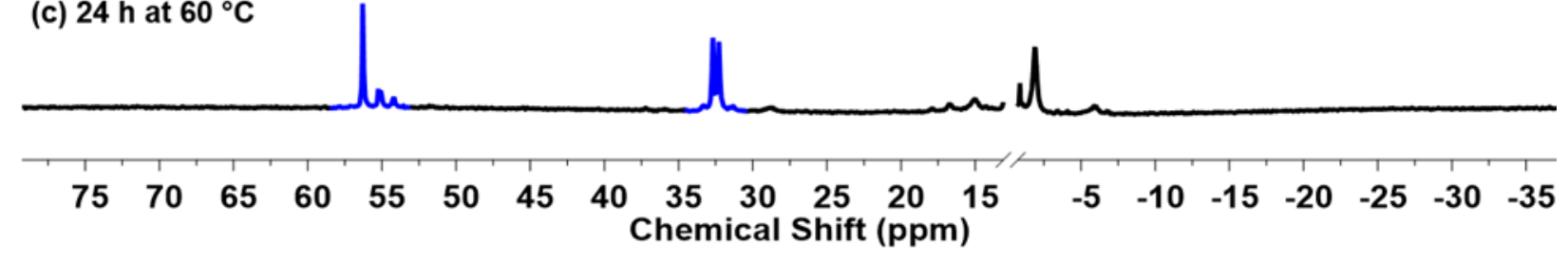

Figure S-7. Reaction progression for the conversion of $\mathbf{8}$ to the corresponding imine complex. 


\section{Binding equilibrium between 11 and alkyl azides.}

Since $\mathbf{1 1}$ is more soluble than $\mathbf{1 0}$ in benzene, we used $\mathbf{1 1}$ to study binding dynamics between dimeric complexes and the monomeric organoazide bound species. A solution of 11 (5 mg, 4.8 $\mu \mathrm{mol}$ as a dimeric form) in benzene- $d_{6}$ was added to a solution of 1-azido-4-(tert-butyl)benzene $\left(1.0-20\right.$ equiv) in benzene- $d_{6}$. Each reaction mixture was transferred to a J. Young NMR tube and the chemical shifts were tracked by ${ }^{1} \mathrm{H}$ NMR spectroscopy.

$\left[\left({ }^{t \mathrm{Bu}} \mathrm{L}\right) \mathrm{CoBr}\right]_{2}$

(11)

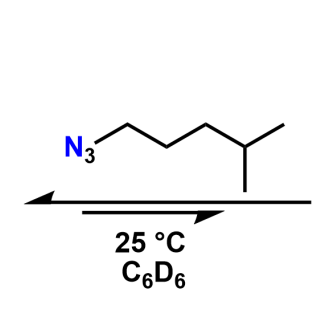

$(17-\mathrm{Br})$

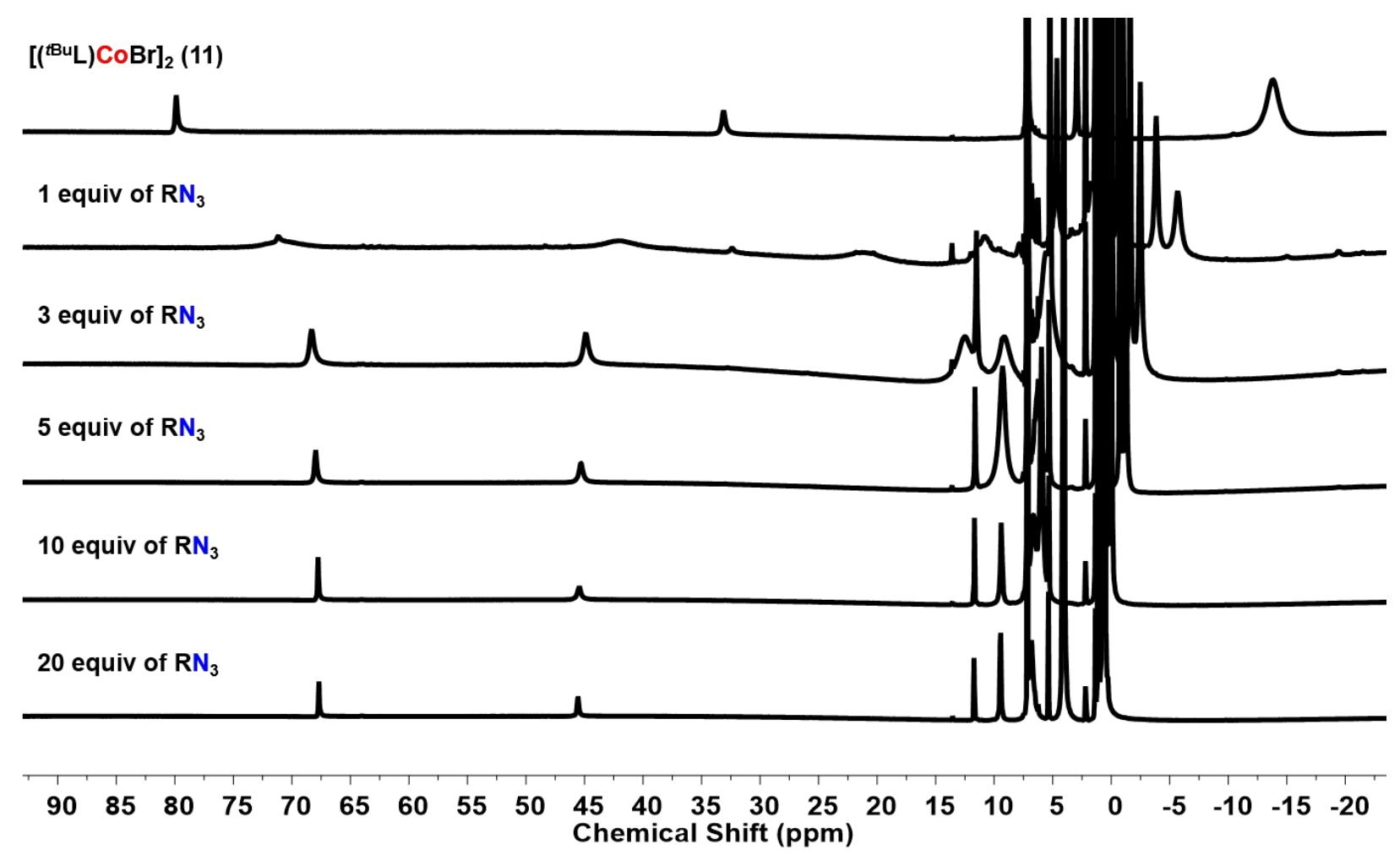

Figure S-8. $600 \mathrm{MHz}{ }^{1} \mathrm{H}$ NMR spectra of $\mathbf{1 7 - B r}$ at varying concentrations of 1-azido-4methylpentane in benzene- $d_{6}$ at $25^{\circ} \mathrm{C}$. 
Job plot analysis. ${ }^{13}$

Solutions of 11 and 1-azido-4-methylpentane $\left(\mathrm{RN}_{3}\right)$ were prepared in benzene- $d_{6}$ and the total concentration of the mixture was maintained constant throughout the analysis. The chemical shifts corresponding to $\mathbf{1 1}$ as a function of the concentration of alkyl azides were measured at $25^{\circ} \mathrm{C}$.

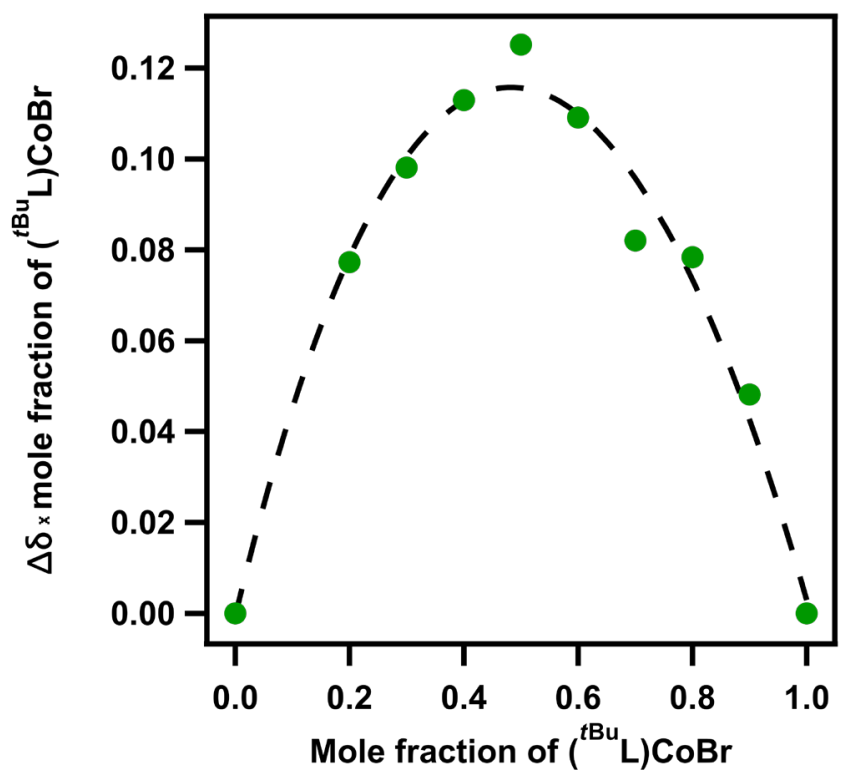

Figure S-9. Job plot obtained at $25^{\circ} \mathrm{C}$ in benzene- $d_{6}$ with $[\mathbf{C o}]+\left[\mathrm{RN}_{3}\right]=8.85 \mathrm{mM}$.

Table S-1. Chemical shifts (ppm) of 11 as a function of $\left[\mathrm{RN}_{3}\right]$ at $25^{\circ} \mathrm{C}$ in benzene- $d_{6}$.

\begin{tabular}{c|ccc}
\hline Entry & $2[11](\mathrm{mM})$ & {$\left[\mathrm{RN}_{3}\right](\mathrm{mM})$} & Chemical shift (ppm) \\
\hline 1 & 8.85 & 0 & 80.69 \\
2 & 7.97 & 0.88 & 80.63 \\
3 & 7.08 & 1.77 & 80.59 \\
4 & 6.20 & 2.66 & 80.57 \\
5 & 5.31 & 3.54 & 80.50 \\
6 & 4.43 & 4.43 & 80.44 \\
7 & 3.54 & 5.31 & 80.40 \\
8 & 2.66 & 6.20 & 80.36 \\
9 & 1.77 & 7.08 & 80.30 \\
10 & 0.88 & 7.97 & 73.71 \\
\hline
\end{tabular}




\section{${ }^{1}$ H NMR titration. ${ }^{14}$}

A solution of 11 was prepared in benzene- $d_{6}$ and its concentration $(1.1 \mathrm{mM})$ was maintained constant throughout the titration with various alkyl azides. The chemical shifts corresponding to 11 as a function of the concentration of alkyl azide were measured at $25^{\circ} \mathrm{C}$ to reveal almost identical binding affinities towards the cobalt center among three different alkyl azide substrates.

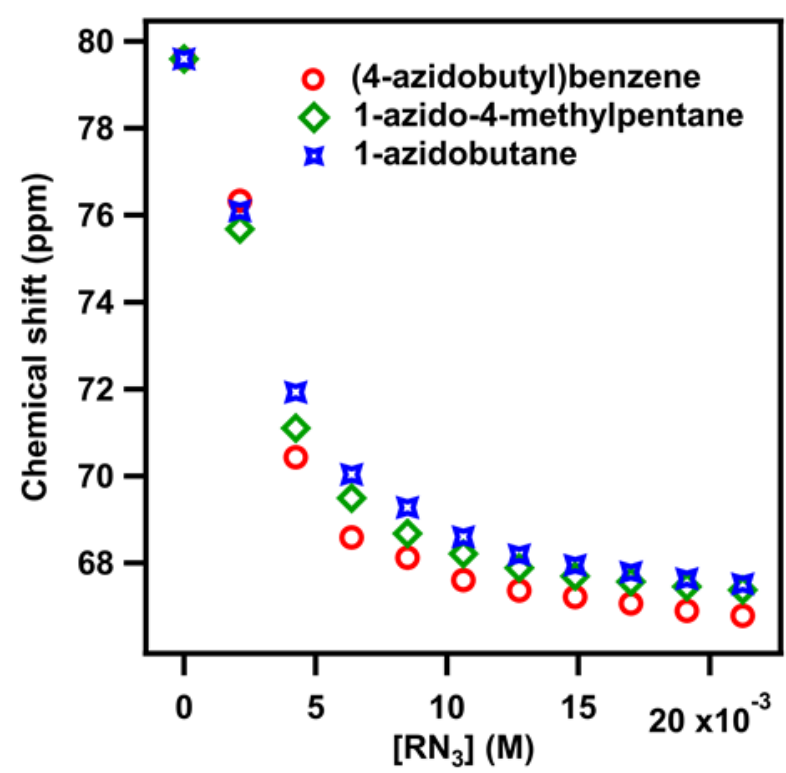

Figure S-10. $600 \mathrm{MHz}{ }^{1} \mathrm{H}$ NMR titration curves of 11 with organoazides. Chemical shifts (ppm) for $\mathbf{1 1}$ as a function of concentration of each azide at $25{ }^{\circ} \mathrm{C}$ in benzene- $d_{6}$. 


\section{Reactivity studies on 19 with cyclohexene.}

A solution of 19 was prepared by adding 1.0 equivalent of 1 -azidobutane to 10 in benzene- $d_{6}$. To the stirring solution, 10 equivalents of 1,4-cyclohexadiene was added. The reaction mixture was transferred to a J. Young NMR tube and heated to $40{ }^{\circ} \mathrm{C}$ for $24 \mathrm{~h}$ to observe formation of 21 .

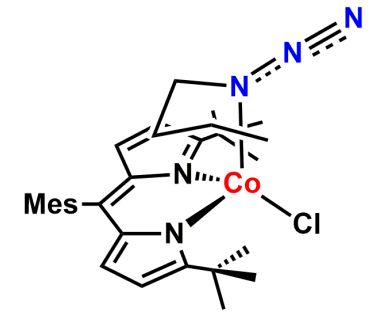

(19)

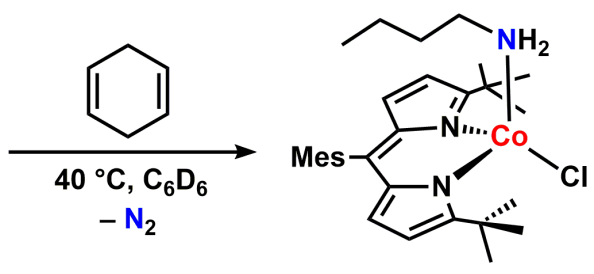

(21)

(a) Complex (19)

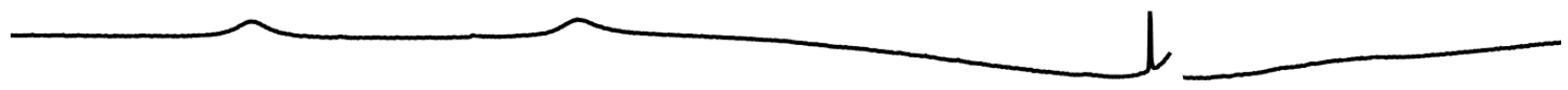

(b) Complex (19) + 1,4-CHD at $40^{\circ} \mathrm{C}, 24 \mathrm{~h}$

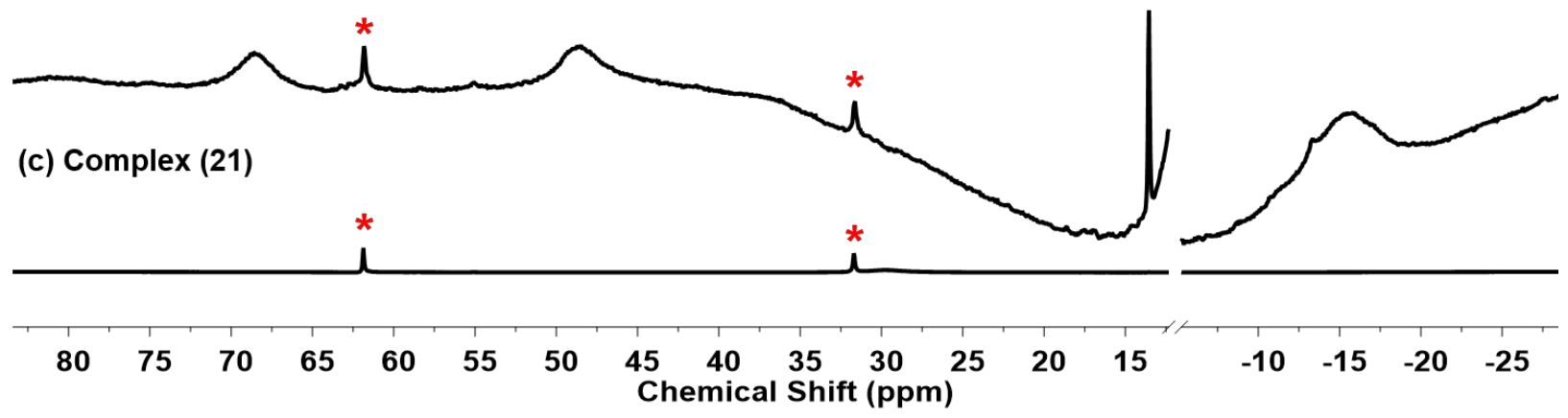

Figure S-11. Reaction progression for the conversion of 19 to 21 in benzene- $d_{6}$ at $40{ }^{\circ} \mathrm{C}$. 


\section{${ }^{1}$ H NMR Spectra of Metal Compounds.}
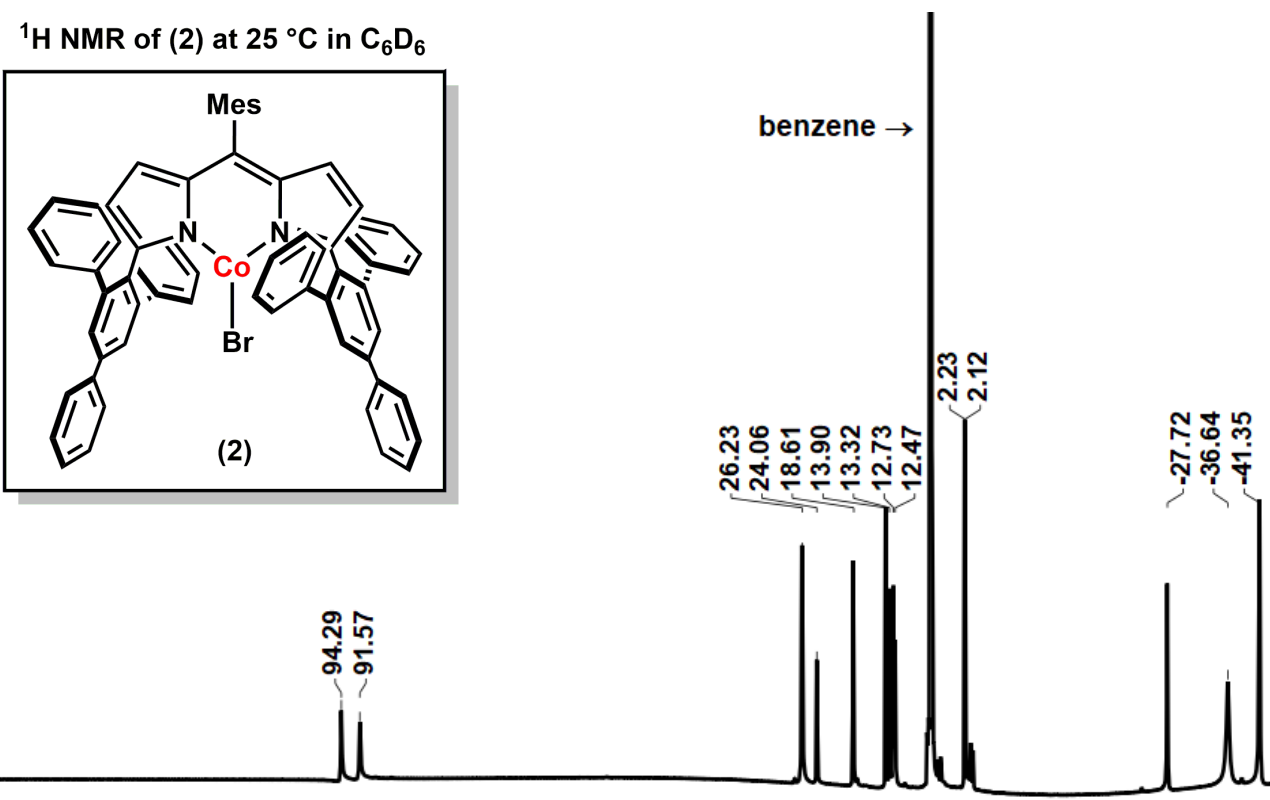

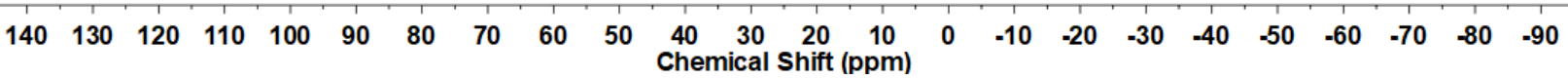

Figure S-12. $500 \mathrm{MHz}{ }^{1} \mathrm{H}$ NMR spectrum of $\left({ }^{\mathrm{Ar}} \mathrm{L}\right) \mathrm{CoBr}(2)$ in benzene- $d_{6}$.

${ }^{1} \mathrm{H}$ NMR of (3) at $25^{\circ} \mathrm{C}$ in $\mathrm{C}_{6} \mathrm{D}_{6}$
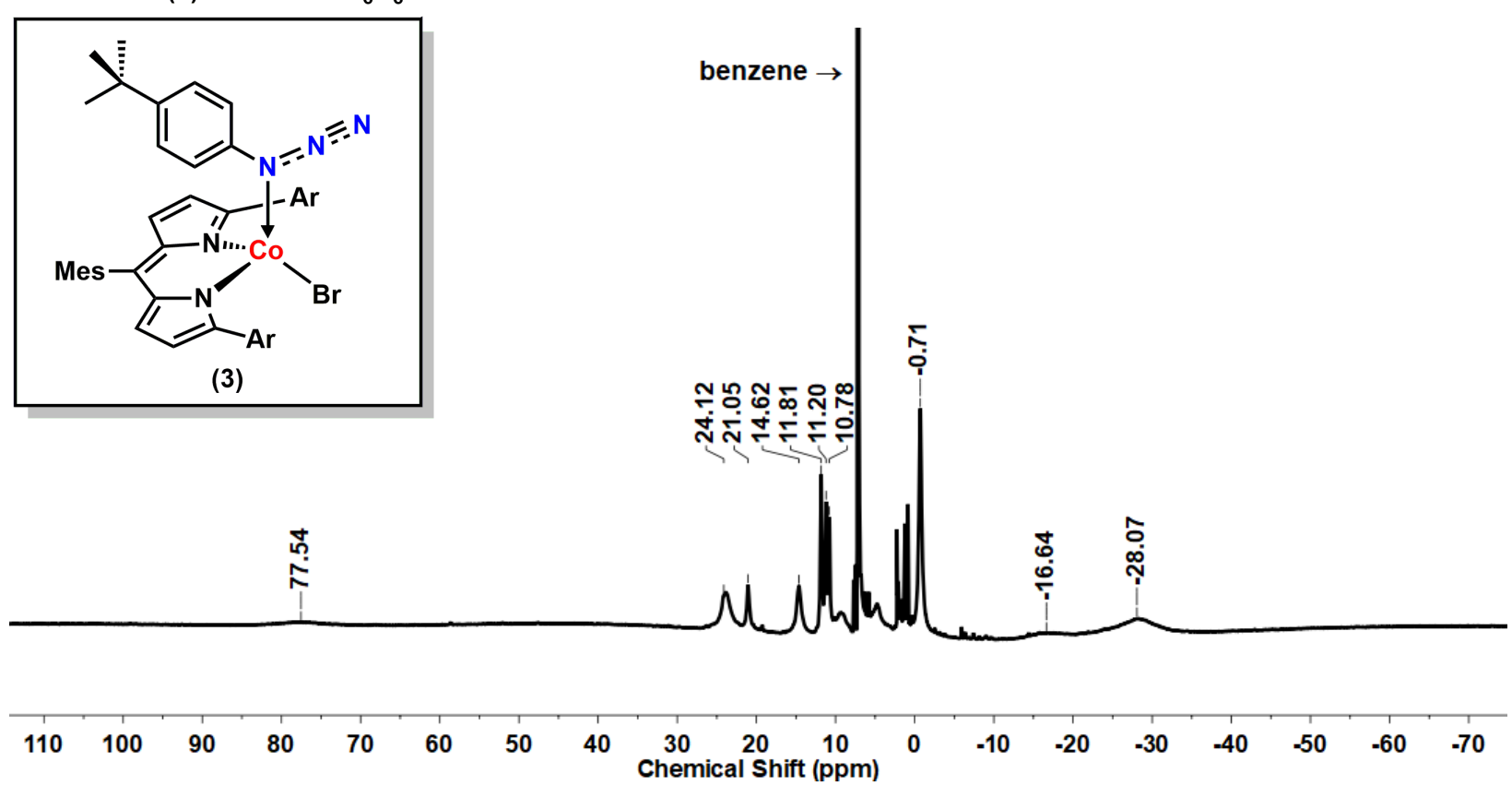

Figure S-13. $500 \mathrm{MHz}{ }^{1} \mathrm{H}$ NMR spectrum of $\left({ }^{\mathrm{Ar}} \mathrm{L}\right) \mathrm{CoBr}\left(\mathrm{N}_{3}\left(\mathrm{C}_{6} \mathrm{H}_{4}-p-{ }^{t} \mathrm{Bu}\right)\right)(3)$ in benzene- $d_{6}$. 

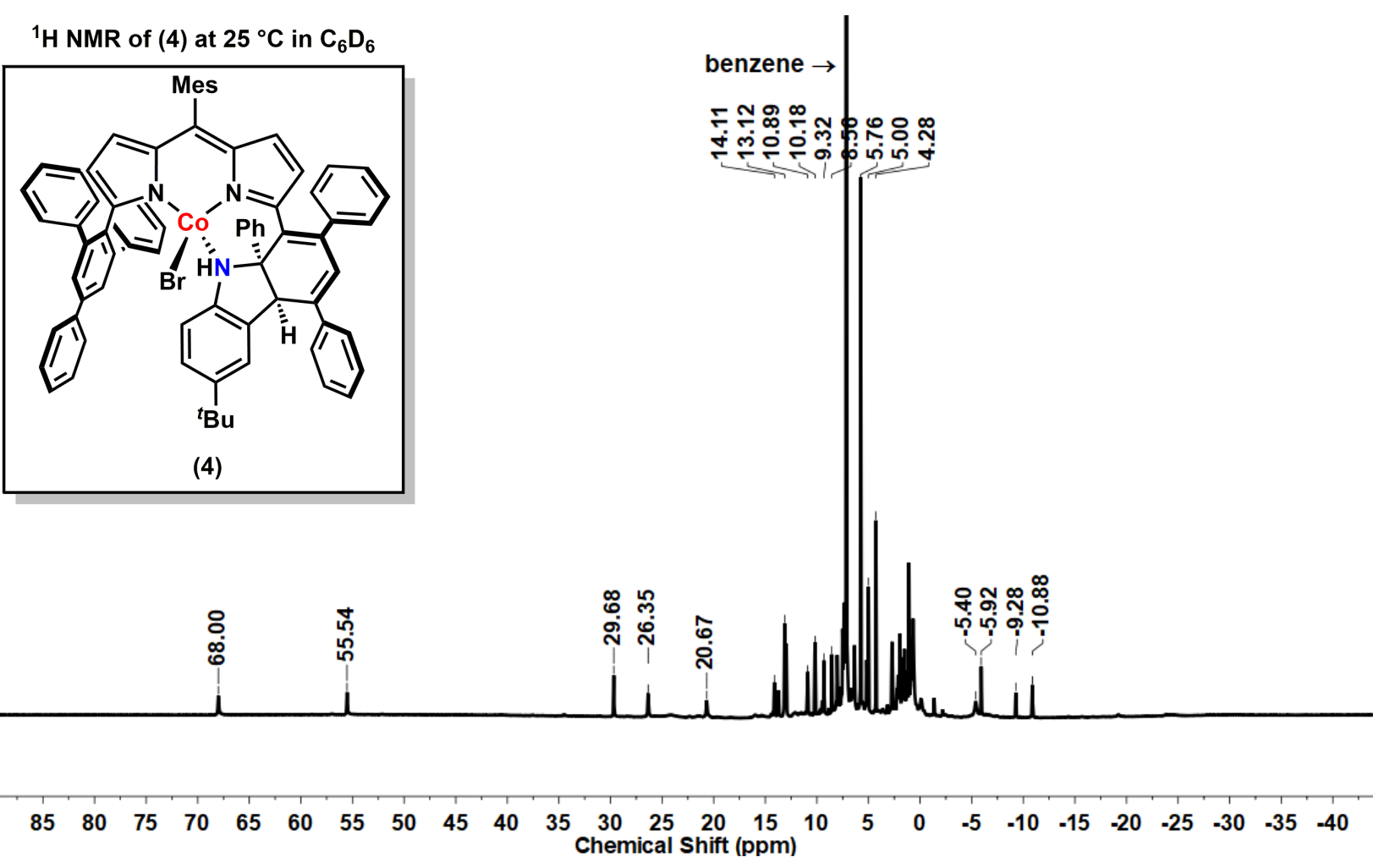

Figure S-14. $500 \mathrm{MHz}{ }^{1} \mathrm{H}$ NMR spectrum of $\left({ }^{\mathrm{Ar}} \mathrm{L}\right) \mathrm{CoBr}([3+2]$ annulation $)$ (4) in benzene- $d_{6}$.

${ }^{1} \mathrm{H}$ NMR of $(5)$ at $25^{\circ} \mathrm{C}$ in $\mathrm{C}_{6} \mathrm{D}_{6}$

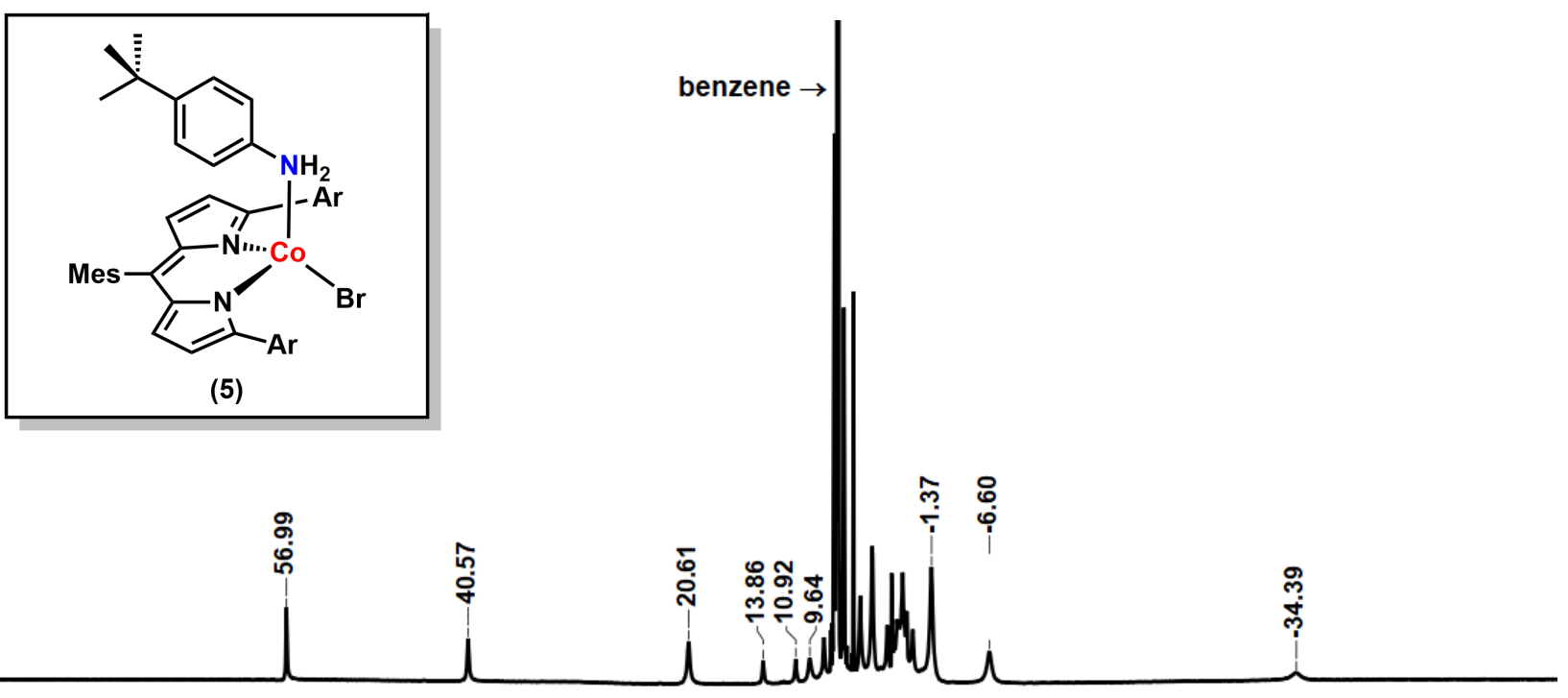

$\begin{array}{llllllllllllllllllllllllllll}80 & 75 & 70 & 65 & 60 & 55 & 50 & 45 & 40 & 35 & 30 & 25 & 20 & 15 & 10 & \begin{array}{c}20 \\ \text { Chemical Shift (ppm) }\end{array} & 0 & -5 & -10 & -15 & -20 & -25 & -30 & -35 & -40 & -45 & -50 & -55\end{array}$

Figure S-15. $500 \mathrm{MHz}{ }^{1} \mathrm{H}$ NMR spectrum of ( $\left.{ }^{\mathrm{Ar}} \mathrm{L}\right) \mathrm{CoBr}\left(\mathrm{NH}_{2}\left(\mathrm{C}_{6} \mathrm{H}_{4}-p-{ }^{t} \mathrm{Bu}\right)\right)(5)$ in benzene- $d_{6}$. 
${ }^{1} \mathrm{H}$ NMR of (6), (7), and (8) at $25^{\circ} \mathrm{C}$ in $\mathrm{C}_{6} \mathrm{D}_{6}$

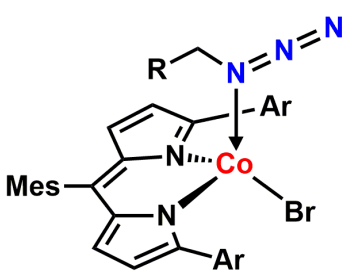

(6) $\mathrm{R}=\mathrm{Ph}$

(7) $\mathrm{R}=\left(\mathrm{CH}_{2}\right)_{3} \mathrm{Ph}$

(8) $\mathrm{R}=\left(\mathrm{CH}_{2}\right)_{2} \mathrm{CH}\left(\mathrm{CH}_{3}\right)_{2}$

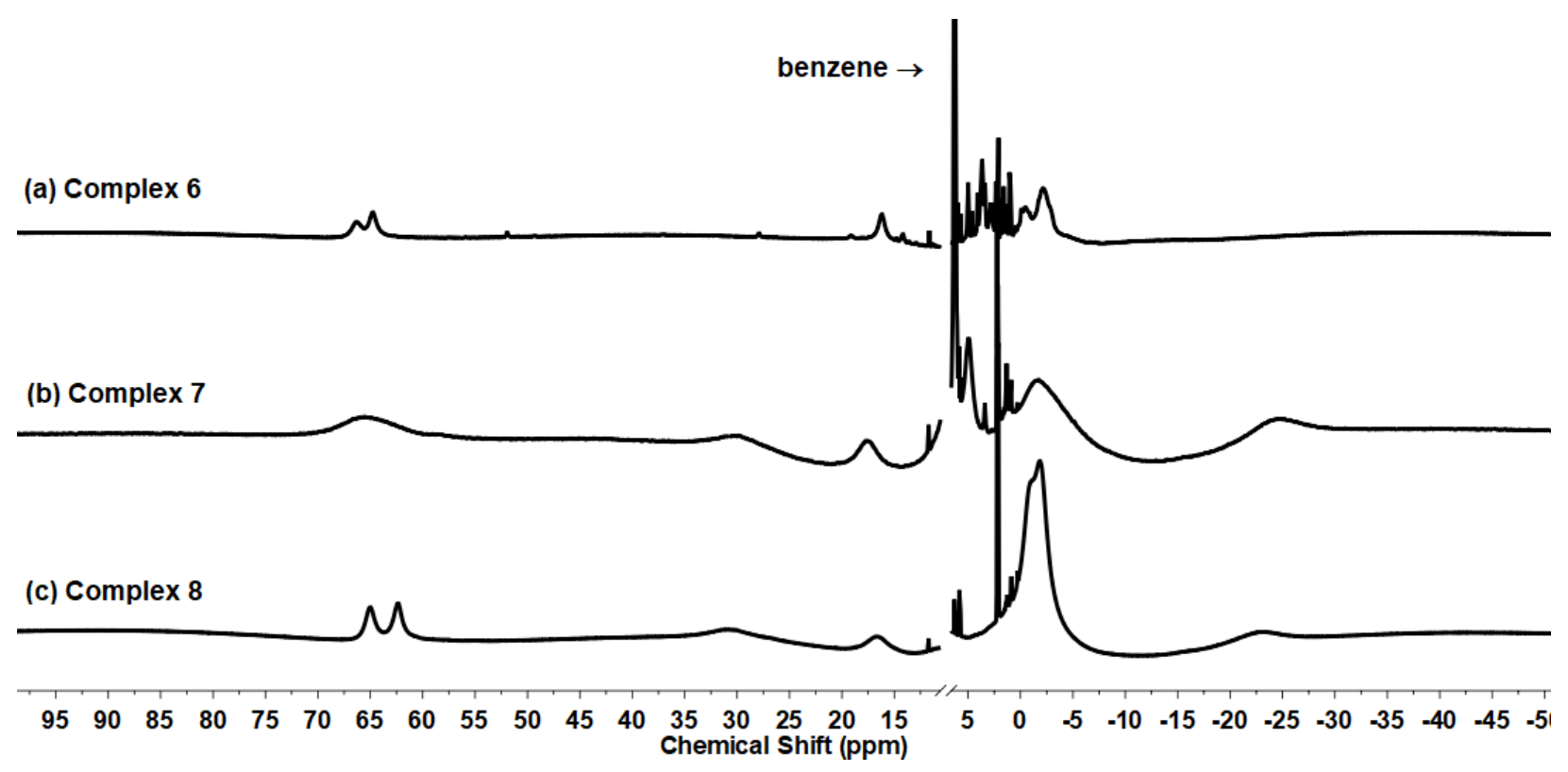

Figure S-16. $500 \mathrm{MHz}{ }^{1} \mathrm{H}$ NMR spectra of $\left({ }^{\mathrm{Ar}} \mathrm{L}\right) \mathrm{CoBr}\left(\mathrm{N}_{3} \mathrm{CH}_{2} \mathrm{R}\right)\left(\mathbf{6}, \mathbf{7}\right.$, and 8) in benzene- $d_{6}$. 

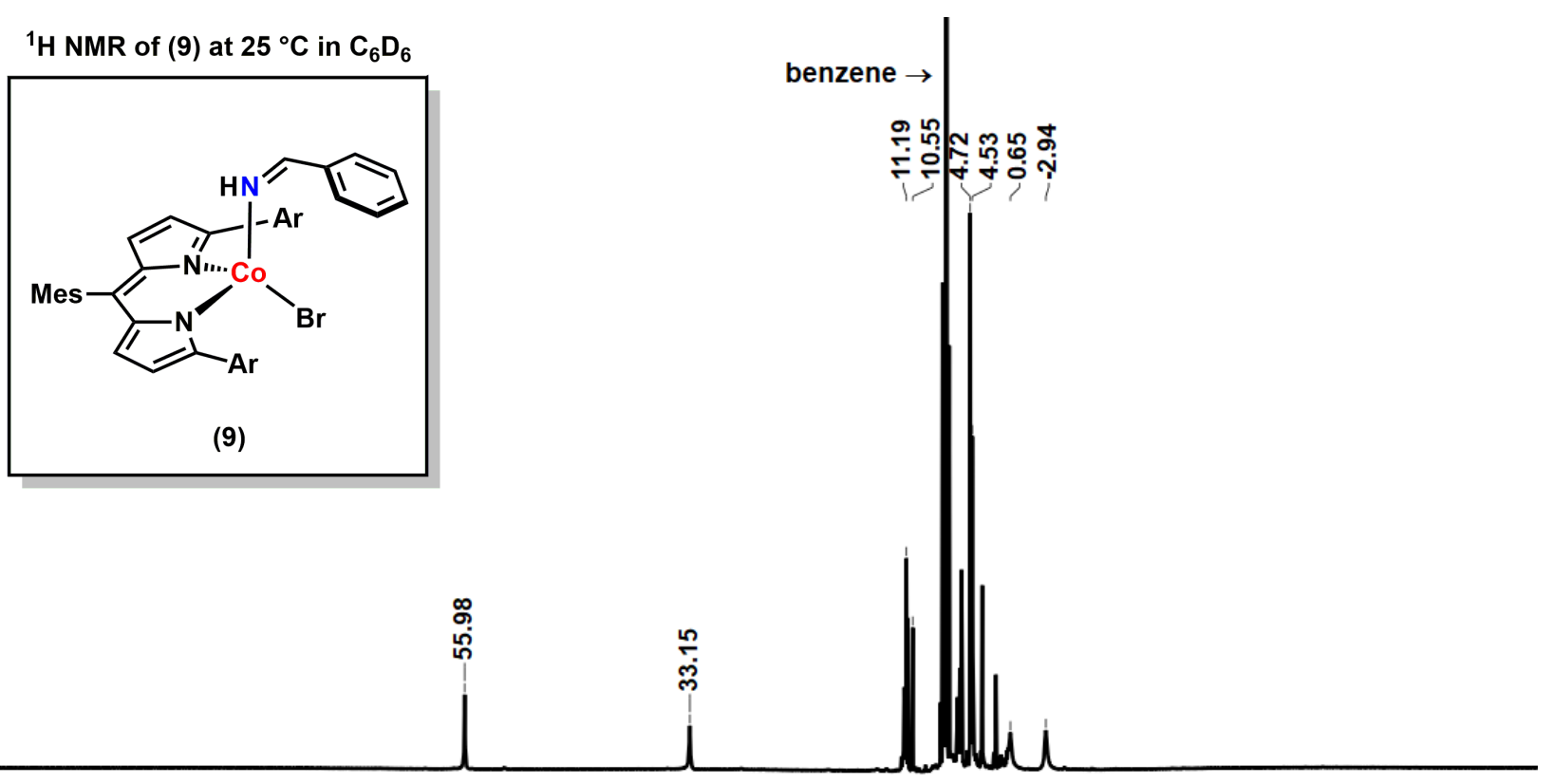

\begin{tabular}{lllllllllllll}
\hline 100 & 90 & 80 & 70 & 60 & 50 & $40 \underset{\text { Chemical Shift (ppm) }}{30} \underset{20}{10}$ & 0 & -10 & -20 & -30 & -40 & -50
\end{tabular}

Figure S-17. $500 \mathrm{MHz}{ }^{1} \mathrm{H}$ NMR spectrum of $\left({ }^{\mathrm{Ar}} \mathrm{L}\right) \mathrm{CoBr}\left(\mathrm{NHCHC}_{6} \mathrm{H}_{5}\right)(\mathbf{9})$ in benzene- $d_{6}$.

${ }^{1} \mathrm{H}$ NMR of $(10)$ and $(11)$ at $25^{\circ} \mathrm{C}$ in $\mathrm{C}_{6} \mathrm{D}_{6}$

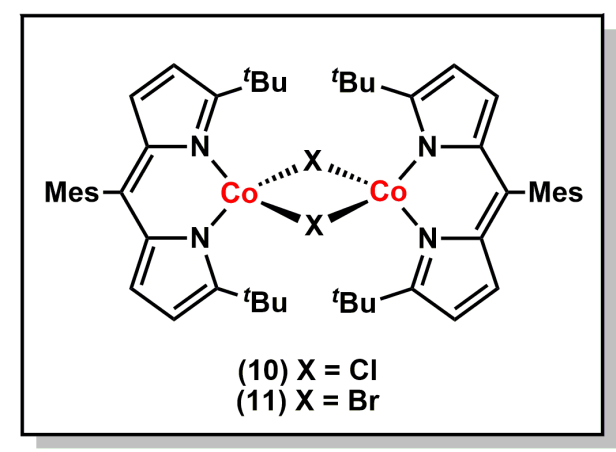

(a) Complex 10

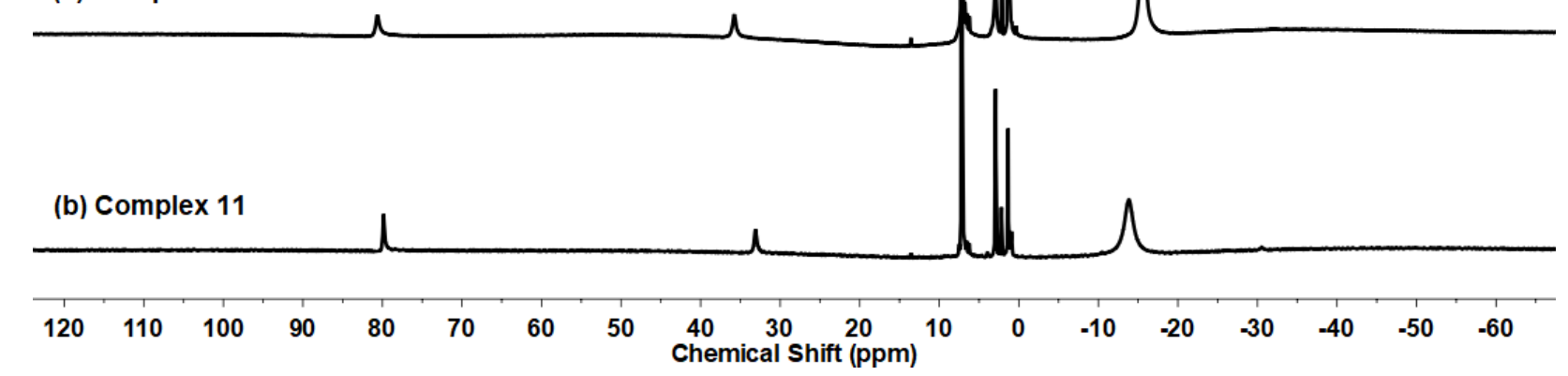

Figure S-18. $500 \mathrm{MHz}{ }^{1} \mathrm{H}$ NMR spectra of (a) $\left[\left({ }^{t \mathrm{Bu}} \mathrm{L}\right) \mathrm{CoCl}\right]_{2}(\mathbf{1 0})$ and $\left.(\mathrm{b})\left[{ }^{t \mathrm{Bu}} \mathrm{L}\right) \mathrm{CoBr}\right]_{2}(\mathbf{1 1})$ in benzene- $d_{6}$. 

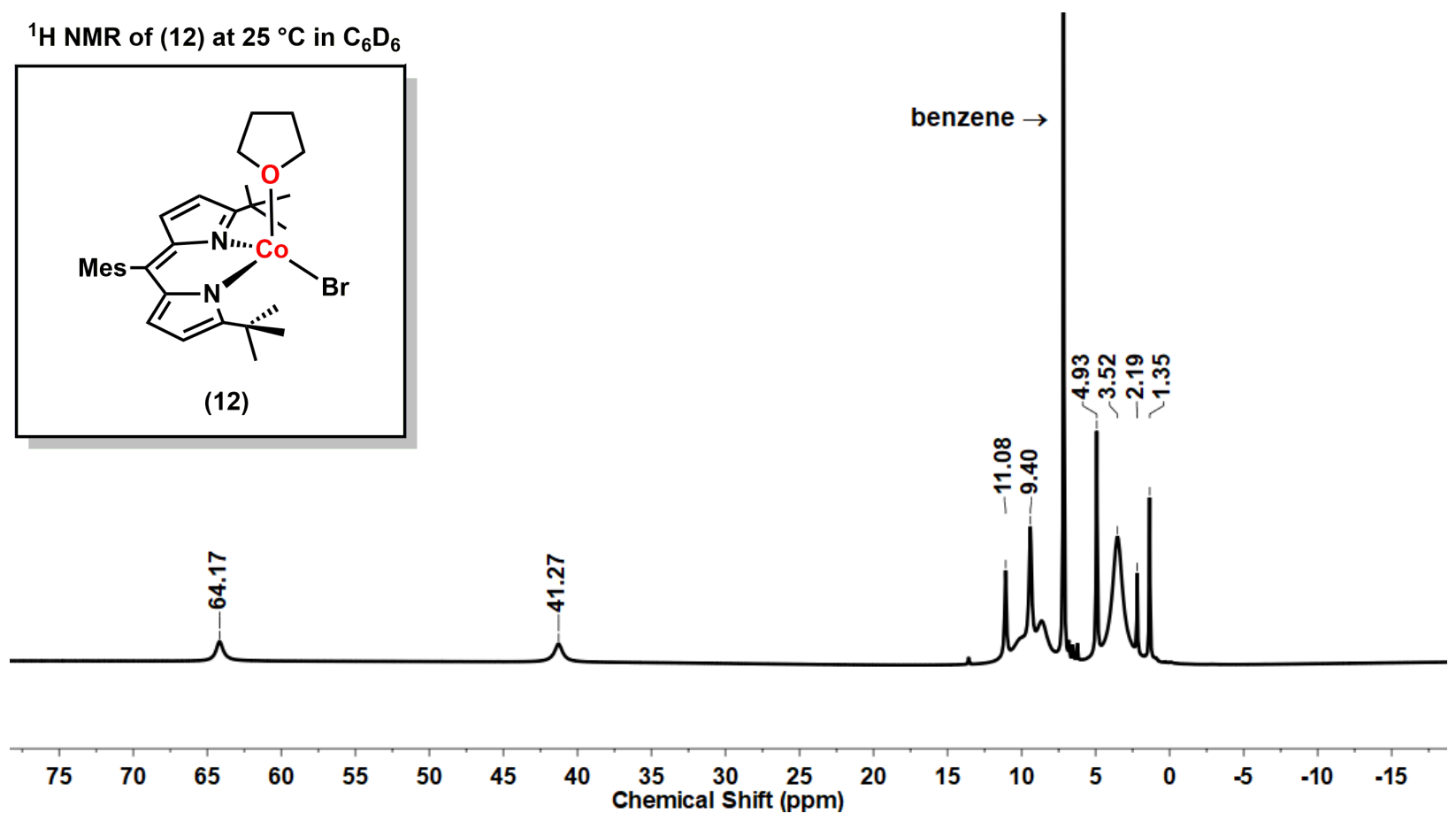

Figure S-19. $500 \mathrm{MHz}{ }^{1} \mathrm{H}$ NMR spectrum of $\left({ }^{t \mathrm{Bu}} \mathrm{L}\right) \mathrm{CoBr}(\mathrm{thf})(\mathbf{1 2})$ in benzene- $d_{6}$.

${ }^{1} \mathrm{H}$ NMR of (14) at $25^{\circ} \mathrm{C}$ in $\mathrm{C}_{6} \mathrm{D}_{6}$

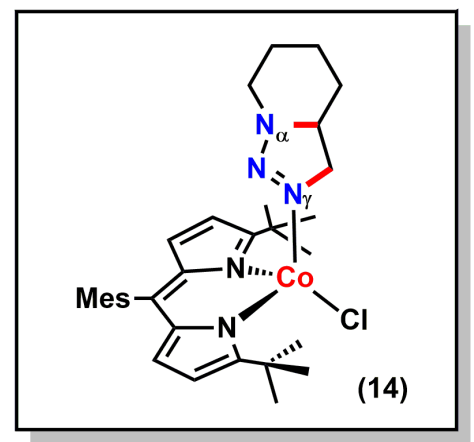

85

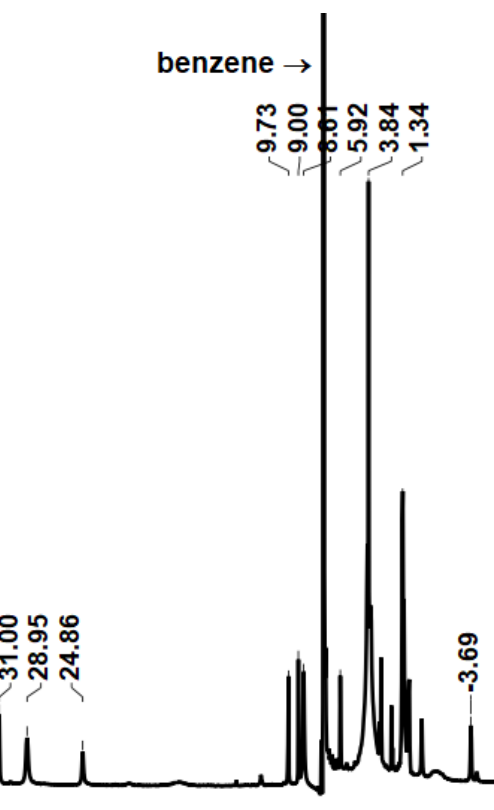

Figure S-20. $500 \mathrm{MHz}{ }^{1} \mathrm{H}$ NMR spectrum of $\left({ }^{t \mathrm{Bu}} \mathrm{L}\right) \mathrm{CoCl}(1,2,3$-dihydrotriazole) (14) in benzene$d_{6}$. 
${ }^{1} \mathrm{H}$ NMR of (15), (17), and (19) at $25^{\circ} \mathrm{C}$ in $\mathrm{C}_{6} \mathrm{D}_{6}$

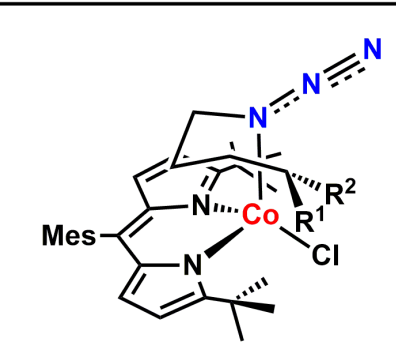

(15) $R^{1}=P h, R^{2}=H$

(17) $R^{1}=M e, R^{2}=M e$

(19) $R^{1}=H, R^{2}=H$

(a) Complex 15

benzene $\rightarrow$

(a) Complex 15

(b) Complex 17

(c) Complex 19

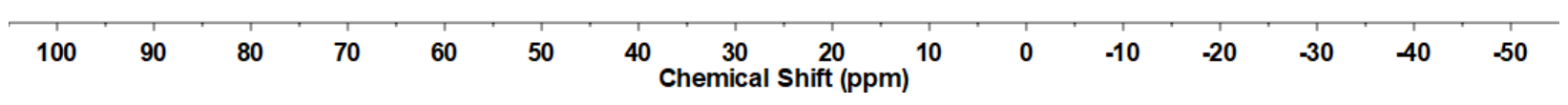

Figure S-21. $500 \mathrm{MHz}{ }^{1} \mathrm{H}$ NMR spectra of $\left({ }^{(\mathrm{Bu}} \mathrm{L}\right) \mathrm{CoCl}\left(\mathrm{N}_{3} \mathrm{R}\right)\left(\mathbf{1 5}, \mathbf{1 7}\right.$, and 19) in benzene- $d_{6}$. 
${ }^{1} \mathrm{H}$ NMR of $(16)$ at $25^{\circ} \mathrm{C}$ in $\mathrm{C}_{6} \mathrm{D}_{6}$

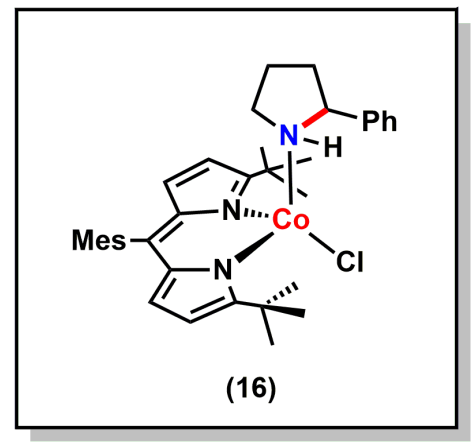

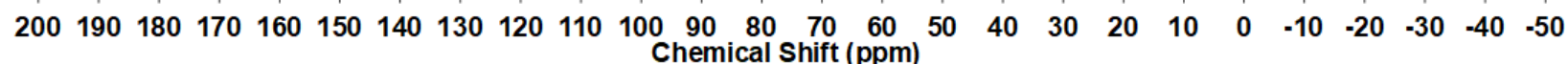

Figure S-22. $500 \mathrm{MHz}{ }^{1} \mathrm{H}$ NMR spectrum of $\left({ }^{t \mathrm{Bu}} \mathrm{L}\right) \mathrm{CoCl}\left(2-\right.$ phenylpyrrolidine) (16) in benzene- $d_{6}$.

${ }^{1} \mathrm{H}$ NMR of $(18)$ at $25^{\circ} \mathrm{C}$ in $\mathrm{C}_{6} \mathrm{D}_{6}$

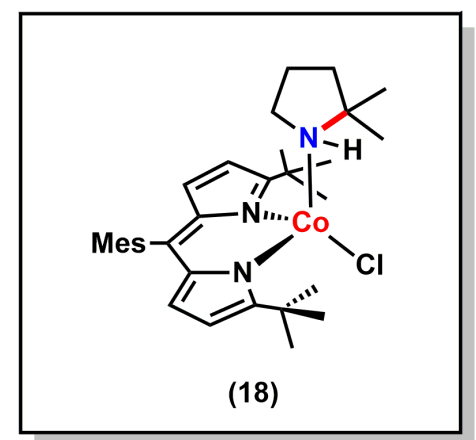

(18)
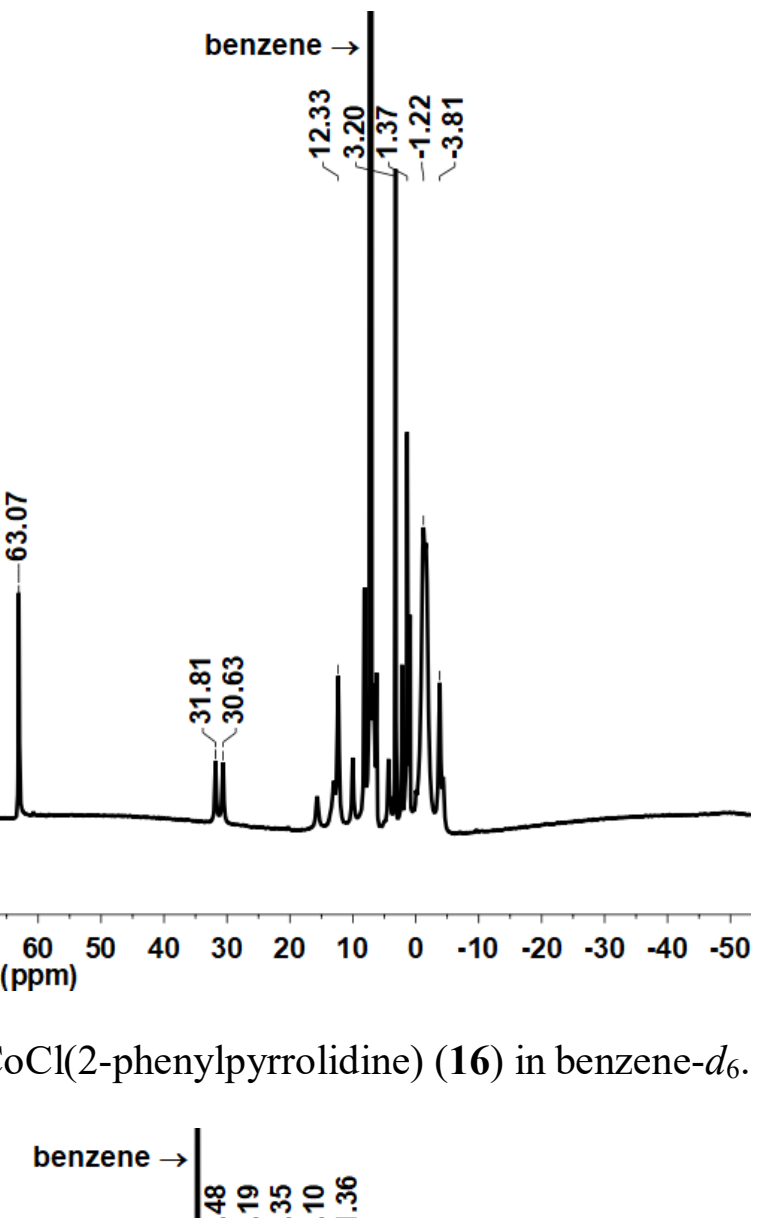

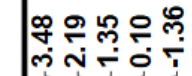

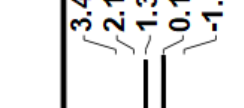

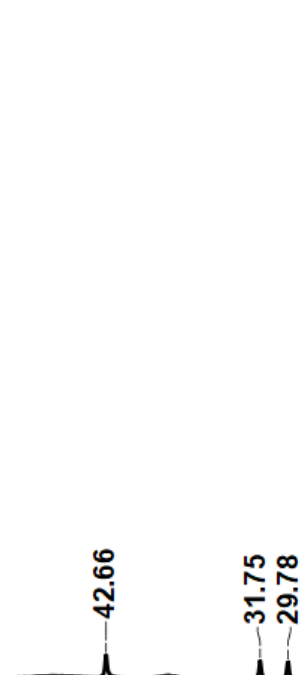
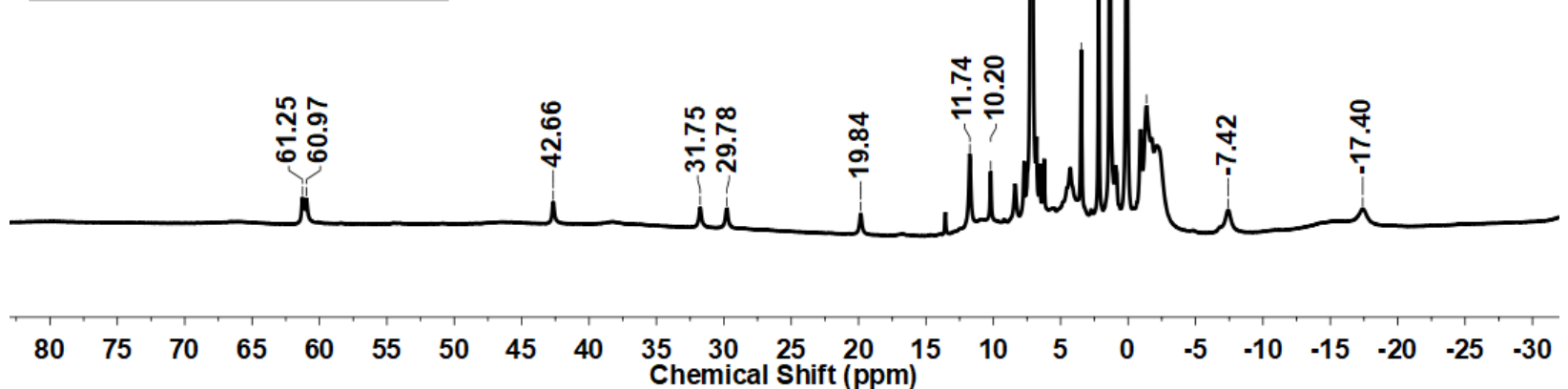

Figure S-23. $500 \mathrm{MHz}{ }^{1} \mathrm{H}$ NMR spectrum of $\left({ }^{t \mathrm{Bu}} \mathrm{L}\right) \mathrm{CoCl}(2,2$-dimethylpyrrolidine) (18) in benzene- $d_{6}$. 
${ }^{1} \mathrm{H}$ NMR of $(20)$ at $25^{\circ} \mathrm{C}$ in $\mathrm{C}_{6} \mathrm{D}_{6}$
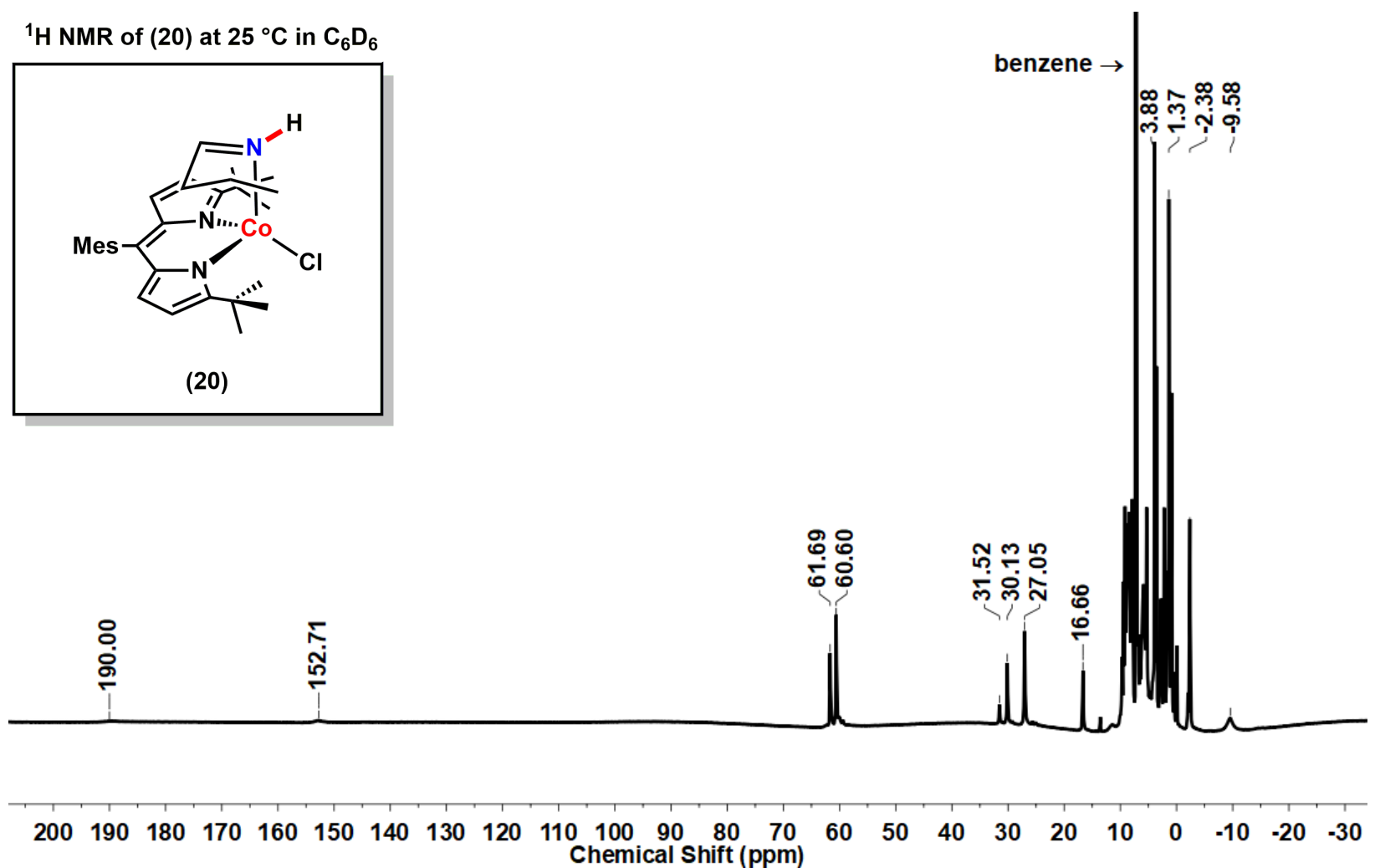

Figure S-24. $500 \mathrm{MHz}{ }^{1} \mathrm{H}$ NMR spectrum of $\left({ }^{t \mathrm{Bu}} \mathrm{L}\right) \mathrm{CoCl}\left(\right.$ butan-1-imine) (20) in benzene- $d_{6}$.

${ }^{1} \mathrm{H}$ NMR of (21) at $25^{\circ} \mathrm{C}$ in $\mathrm{C}_{6} \mathrm{D}_{6}$

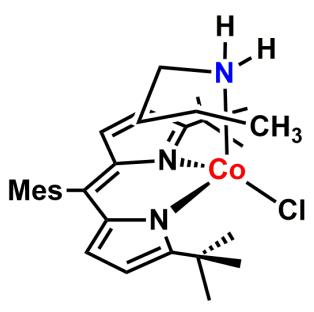

(21)

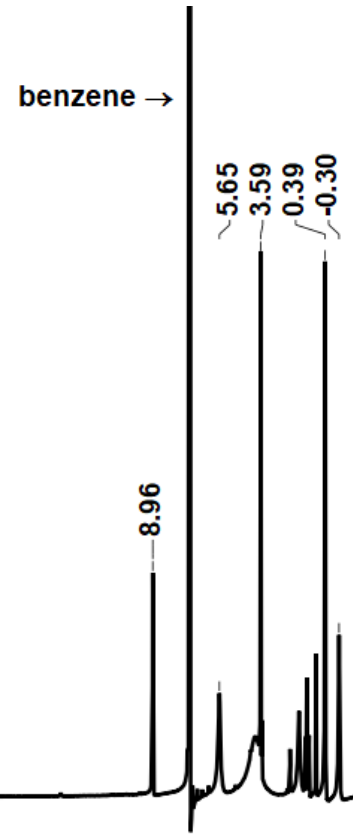

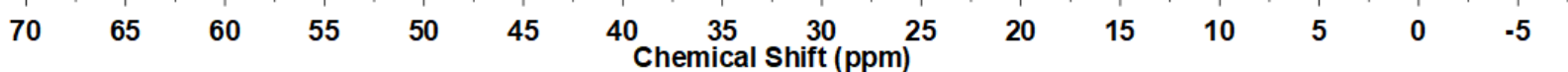

Figure S-25. $500 \mathrm{MHz}{ }^{1} \mathrm{H}$ NMR spectrum of $\left({ }^{t \mathrm{Bu}} \mathrm{L}\right) \mathrm{CoCl}\left(n-\mathrm{BuNH}_{2}\right)(\mathbf{2 1})$ in benzene- $d_{6}$. 


\section{X-Ray Diffraction Techniques.}

General. All structures were collected on a Bruker three-circle platform goniometer equipped with an Apex II CCD and an Oxford cryostream cooling device. Radiation was from a graphite fine focus sealed tube Mo Ka $(0.71073 \AA)$ source. Crystals were mounted on a cryoloop or glass fiber pin using Paratone N oil. Structures were collected at $100 \mathrm{~K}$. Data was collected as a series of $\varphi$ and/or $\omega$ scans. Data was integrated using $\mathrm{SAINT}^{15}$ and scaled with either a numerical or multiscan absorption correction using SADABS. ${ }^{15}$ The structures were solved by intrinsic methods or Patterson maps using SHELXL-2018 ${ }^{16}$ and refined against $F^{2}$ on all data by full matrix least squares with SHELXL-2018. ${ }^{16}$ All non-hydrogen atoms were refined anisotropically. Hydrogen atoms were placed at idealized positions and refined using a riding model. The isotropic displacement parameters of all hydrogen atoms were fixed to 1.2 times the atoms they are linked to (1.5 times for methyl groups). Further details on particular structures are noted below. Please refer to cif files for detail of disorder refinement.

For section 2.4.1. Initial $100 \mathrm{~K}$ structure was solved and refined as described above. For data sets obtained following heating at $345 \mathrm{~K}$, non-H atoms of the product were located in difference Fourier maps, calculated with coefficients $F_{\mathrm{o}}$ (heated) $-F_{\mathrm{o}}($ initial_100 K), and then refined with constraints on the product molecule's atomic displacement parameters to the corresponding values of the reactant molecule (EADP instructions of SHELXL-2018, for the azide adduct and iminyl units). ${ }^{16}$ The percentage of the reactant in the crystal was treated as a variable in the refinements.

More specifically, for the reactive fragment, all non-H atoms of the product were located in the reaction-difference maps, calculated with coefficients $F_{\mathrm{o}}$ (heat)- $F_{\mathrm{o}}$ (initial), and then refined with restraints on the reacted fragment's bond lengths and constraints of the atomic displacement parameters to the corresponding values of the unreacted fragment (SADI and EADP instructions of SHELXL-2018). The restraints of the atomic displacement parameters (SIMU/RIGU instructions of SHELXL-2018) if necessary, have been applied for such disorder refinement. For the non-reactive fragment, the restraints on bond lengths (SADI/SAME instructions of SHELXL2018), as well as the restraints of the atomic displacement parameters, if necessary, have been applied for the disorder refinement. In order to get a reasonable bond length for the severely 
disordered hexane and avoid a non-positive definite of atomic displacement parameters, the restraints of DFIX and ISOR were applied.

For section 2.4.2. The X-ray crystal structures of $\mathbf{7}-\mathbf{N}_{\mathbf{2}}$ and $\mathbf{8}-\mathbf{N}_{\mathbf{2}}$ were obtained from $\mathbf{7}$ and $\mathbf{8}$ respectively, using synchrotron radiation (0.41328 $\AA$ ) at ChemMatCARS located at the Advanced Photon Source housed at Argonne National Lab. The data was collected at $100 \mathrm{~K}$ (Cryojet $\mathrm{N}_{2}$ cold stream) using a vertically mounted Bruker D8 three-circle platform goniometer equipped with a PILATUS3 X CdTe 1M detector. Data was collected as a series of $\varphi$ and/or $\omega$ scans. Data were integrated using SAINT and scaled with a multi-scan absorption correction using SADABS. ${ }^{15}$ Structures were solved by intrinsic phasing using SHELXT (Apex2 program suite v2014.1) and refined against $F^{2}$ on all data by full matrix least squares with SHELXL-2014. All non-hydrogen atoms were refined anisotropically. $\mathrm{H}$ atoms were placed at idealized positions and refined using a riding model. For the refinement of $\mathbf{8}-\mathbf{N}_{\mathbf{2}}$ specifically, a disordered hexanes molecule $(1 / 2$ occupied) was identified in the final stages of refinement. The disorder of the solvent was dealt with by application of the program Squeeze (Spek, A. L. Acta Cryst. 2015, C71, 9-18) as implemented in Platon (Spek, A. L. Acta Cryst. 2009, D65, 148-155) which allows for the mathematical compensation of the electron contribution of disordered solvent contained in the voids to the calculated diffraction intensities.

\section{Interpretation of single-crystal transformation results.}

(1) Both $\mathbf{7}$ and $\mathbf{8}$ are stable at room temperature as supported by clean ${ }^{1} \mathrm{H}$ NMR spectra (Figure S16), proving that there is no loss of $\mathrm{N}_{2}$ and subsequent decomposition to amide, imine, or other unknown spices at room temperature. If $\mathbf{7}$ or $\mathbf{8}$ co-crystallized with either $\mathrm{Co}^{\mathrm{III}}$ amide or $\mathrm{Co}^{\mathrm{II}}$ imine at room temperature, we should see a second set of distinct chemical shifts by ${ }^{1} \mathrm{H}$ NMR spectroscopy. However, those features have not been detected in the ${ }^{1} \mathrm{H}$ NMR of the crystalline material (Figure S-16). Specifically, the corresponding imine complex of $\mathbf{8}$ can be generated only upon heating (above $60{ }^{\circ} \mathrm{C}, 24 \mathrm{~h}$ ) and displays distinct ${ }^{1} \mathrm{H}$ NMR chemical shifts with respect to the azide adduct precursor 8 (Figure S-7).

(2) The chemical composition and purity of $\mathbf{7}$ and $\mathbf{8}$ have also been supported by CHN elemental analysis (SI 8-9). 
(3) Formation of the authentic $\mathrm{Co}^{\mathrm{III}}$ alkyl amide complex $\left({ }^{\mathrm{Ar}} \mathrm{L}\right) \mathrm{CoBr}(\mathrm{NHR})$ via decomposition of $\mathbf{7}$ or $\mathbf{8}$ has proven unsuccessful. Specifically, treatment of $\mathbf{7}$ or $\mathbf{8}$ with excess amount of external H-atom donors (e.g., 1,4-cyclohexadiene) shows no reactivity at room temperature. This lack of intermolecular $\mathrm{H}$-atom abstraction (HAA) reactivity has been stated in section 2.4 and the conclusion section to highlight the differences in reactivity between the alkyl azide adducts ( 7 and 8) and the analogous aryl azide adduct 3 . We note that independent synthesis of the authentic amide complexes from $\left({ }^{\mathrm{Ar}} \mathrm{L}\right) \mathrm{CoBr}(2)$ via salt-metathesis or stepwise deprotonation/oxidation has not been successful either, suggesting that formation of a $\mathrm{Co}^{\mathrm{III}}$ amide complex is highly unlikely.

(4) In addition to the absence of intermolecular HAA reactivity, intramolecular HAA pathways to generate a $\mathrm{Co}^{\mathrm{III}}$ amide complex directly from the azide adduct precursor (e.g., 7 or 8 ) can be ruled out by considering the mechanism of an intramolecular 1,2-hydrogen shift observed for 8 (for literature supporting that an $\alpha-\mathrm{H}$ atom participates in formation of imine products from metalnitrene precursors: J. Am. Chem. Soc., 2019, 141 (19), 7797-7806). Given that the imine adduct is the exclusive product upon thermal decomposition of $\mathbf{8}$ via the intramolecular 1,2-hydrogen shift (Figure S-7), the corresponding $\mathrm{Co}^{\mathrm{III}}$ amide complex cannot even exist as an intermediate, unless the $\mathrm{H}$-atom shift is stepwise, which accompanies formation of a highly unstable secondary carboradical on the $\mathrm{C} 1$ ( or $\mathrm{C} \alpha$ ) position. To the best of our knowledge, there is no literature precedent supporting the presence of such an unstable intermediate. Therefore, it is impractical that such a synthetically inaccessible molecule can be naturally generated and even co-crystallized with 7 or 8 within the same crystalline lattice at room temperature. 
Table S-2. Changes in occupancy of nitrogen atoms for 8 . The initial occupancy values for $\mathrm{N}_{\beta}$ and $\mathrm{N} \gamma$ atoms of $\mathbf{8}$ (dark structure, before heating) were set to 1 for the purpose of comparing the relative changes in the occupancies before and after heating. As such, the noted occupancy values indicate relative conversions.

\begin{tabular}{|c|c|c|c|}
\hline & $\mathrm{N}_{\alpha}$ & $\mathrm{N}_{\beta}$ & $\mathrm{N}_{\gamma}$ \\
\hline Initial_100 K & 1.000 & 1.000 & 1.000 \\
\hline $1^{\text {st }}$ heating & $0.839(4), 0.161(4)$ & $0.839(4)$ & $0.839(4)$ \\
\hline $2^{\text {nd }}$ heating & $0.829(4), 0.171(4)$ & $0.829(4)$ & $0.829(4)$ \\
\hline $3^{\text {rd }}$ heating & $0.810(4), 0.190(4)$ & $0.810(4)$ & $0.810(4)$ \\
\hline $4^{\text {th }}$ heating & $0.797(4), 0.203(4)$ & $0.797(4)$ & $0.797(4)$ \\
\hline
\end{tabular}



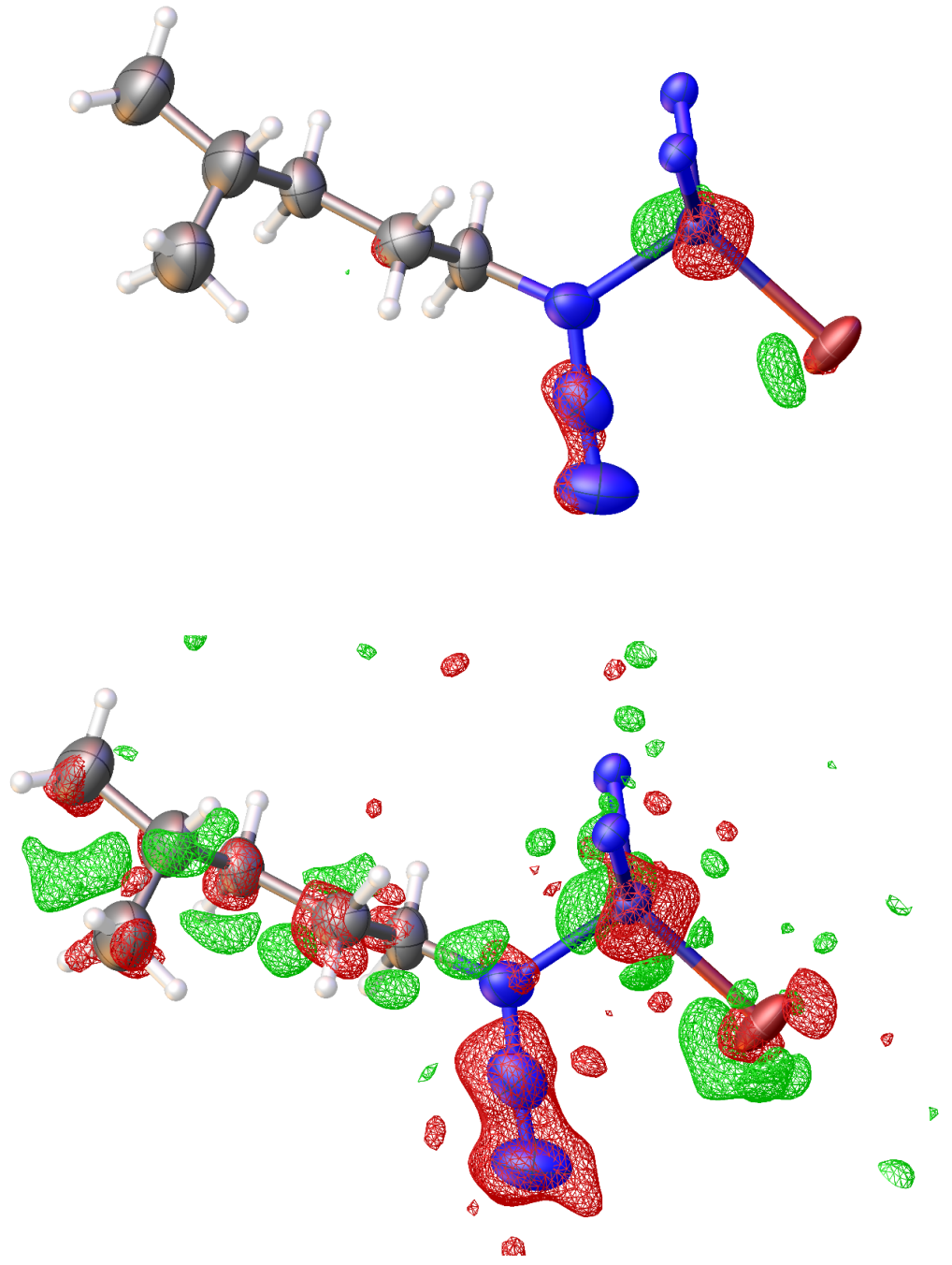

Figure S-26. Reaction-difference map obtained for complex 8 at $100 \mathrm{~K}$ following four iterations of heating to $345 \mathrm{~K}$ (8-heat4) (top: at 1.2 electron density level, bottom: at 0.6 electron density level). Green and red colors represent positive- and negative electron densities, respectively. 


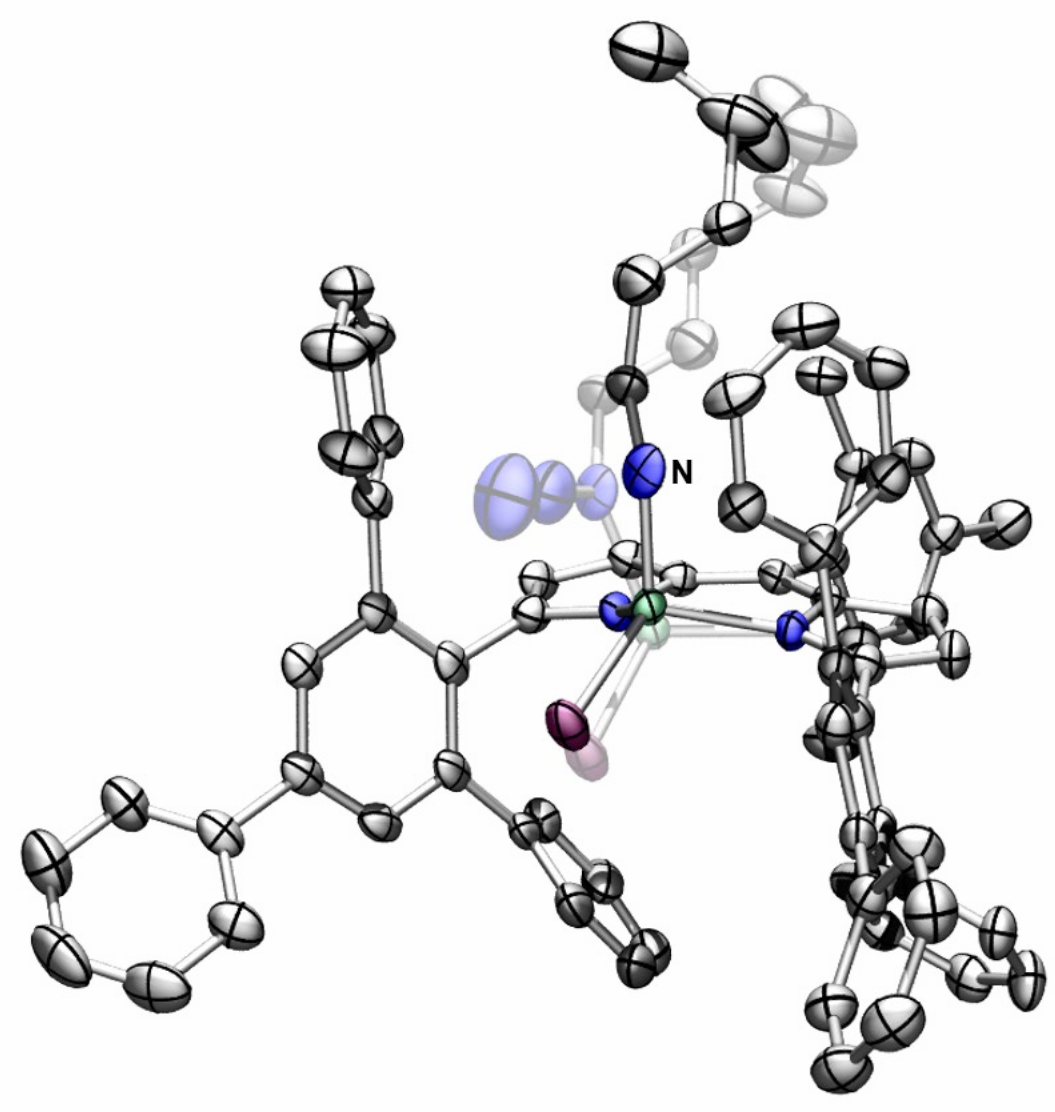

Figure S-27. Solid-state molecular structure for $\mathbf{8}-\mathbf{N}_{\mathbf{2}}$ (8-heat4). Thermal ellipsoid plot showing the dark structure (faded) and the heated structure (bold) generated from complex 8. 


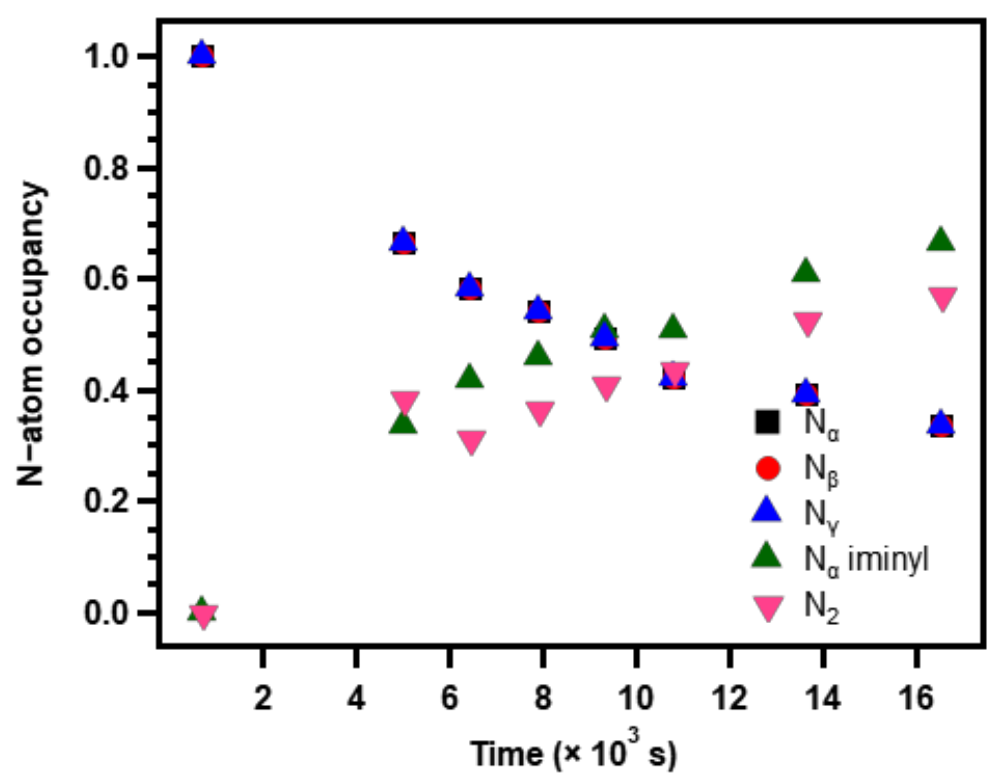

Figure S-28. Changes in occupancy of the organoazide $\mathrm{N}$-atoms in 7 during synchrotron irradiation $(\lambda=0.41328 \AA)$.

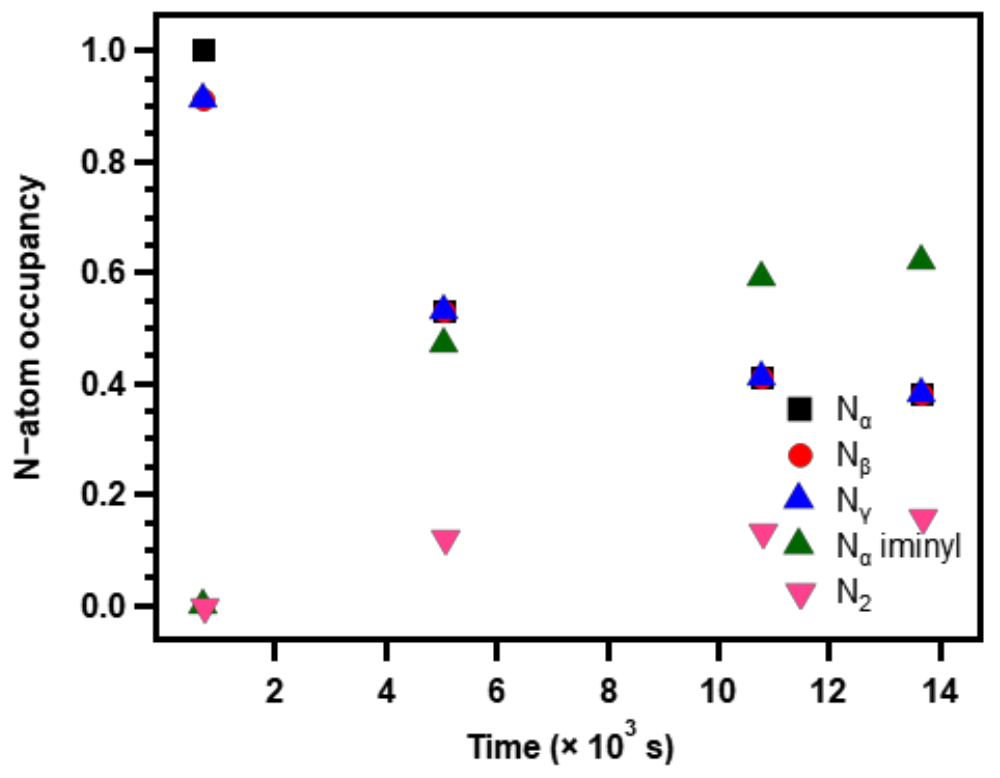

Figure S-29. Changes in occupancy of the organoazide $\mathrm{N}$-atoms in $\mathbf{8}$ during synchrotron irradiation $(\lambda=0.41328 \AA)$. 

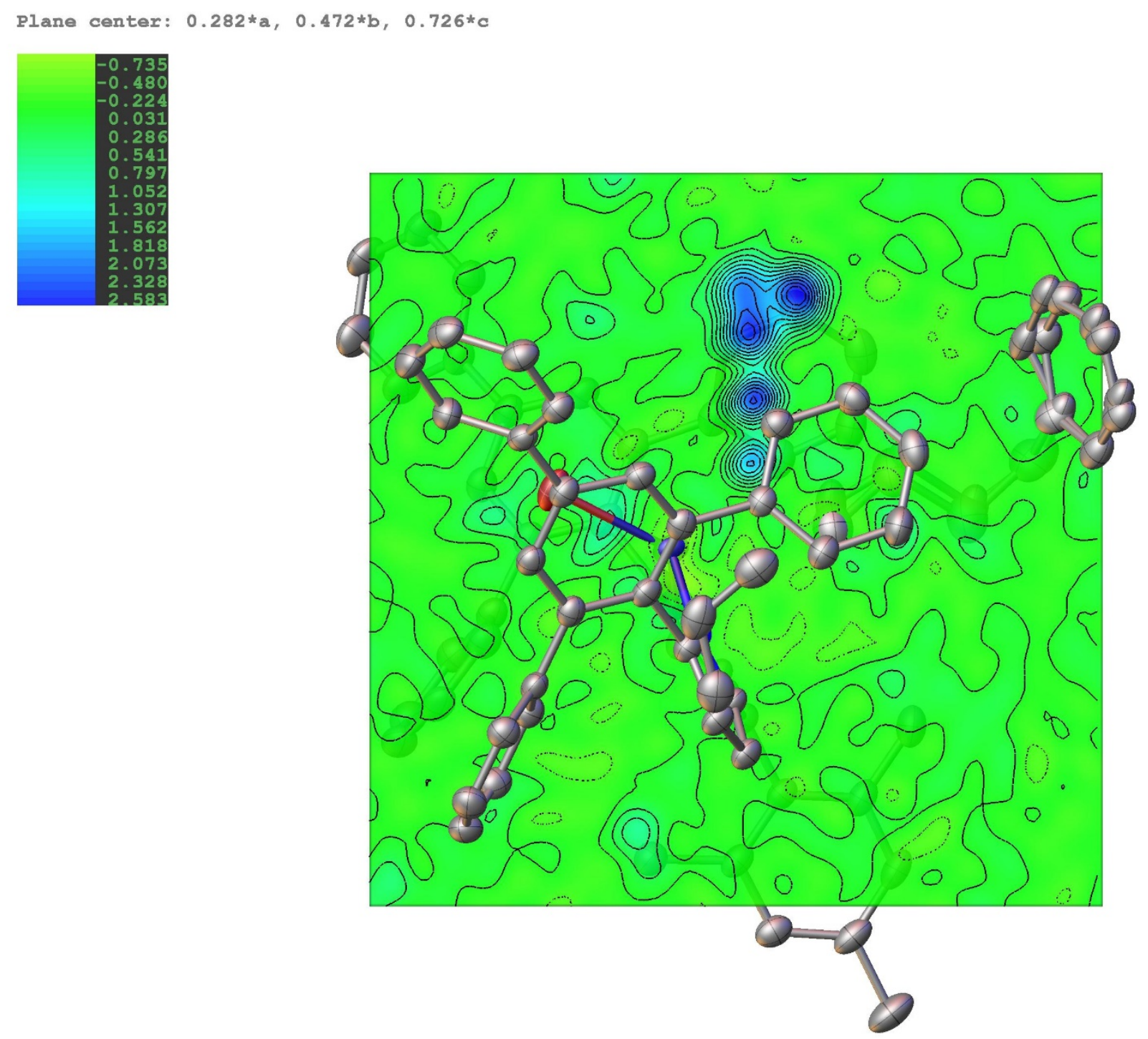

Figure S-30. Electron density map for $\mathbf{7}-\mathbf{N}_{2}$, which illustrates that the electron density of the residual azide (7) is co-localized with the electron density of the $\mathrm{N}_{2}$ fragment. Due to this effective compositional disorder, we restrained the metrics of the residual 7 and the $\mathrm{N}_{2}$ molecule in order to obtain chemically meaningful metrics for $\mathbf{7}-\mathbf{N}_{\mathbf{2}}$. 

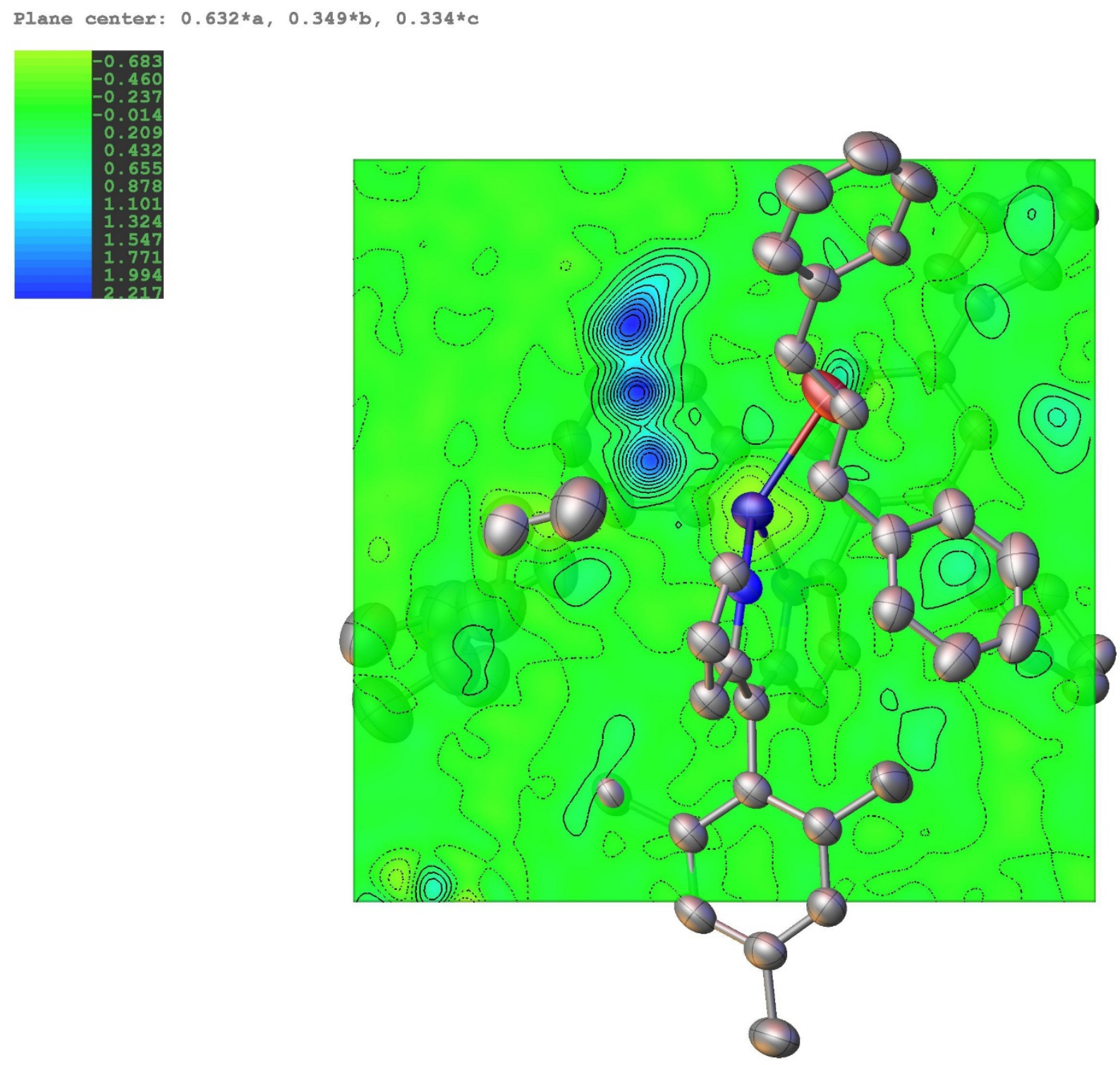

Figure S-31. Electron density map for $\mathbf{8}-\mathbf{N}_{2}$, which illustrates that the electron density of the residual azide (8) is co-localized with the electron density of the $\mathrm{N}_{2}$ fragment. Due to this effective compositional disorder, we restrained the metrics of the residual 8 and the $\mathrm{N}_{2}$ molecule in order to obtain chemically meaningful metrics for $\mathbf{8}-\mathbf{N}_{2}$. 


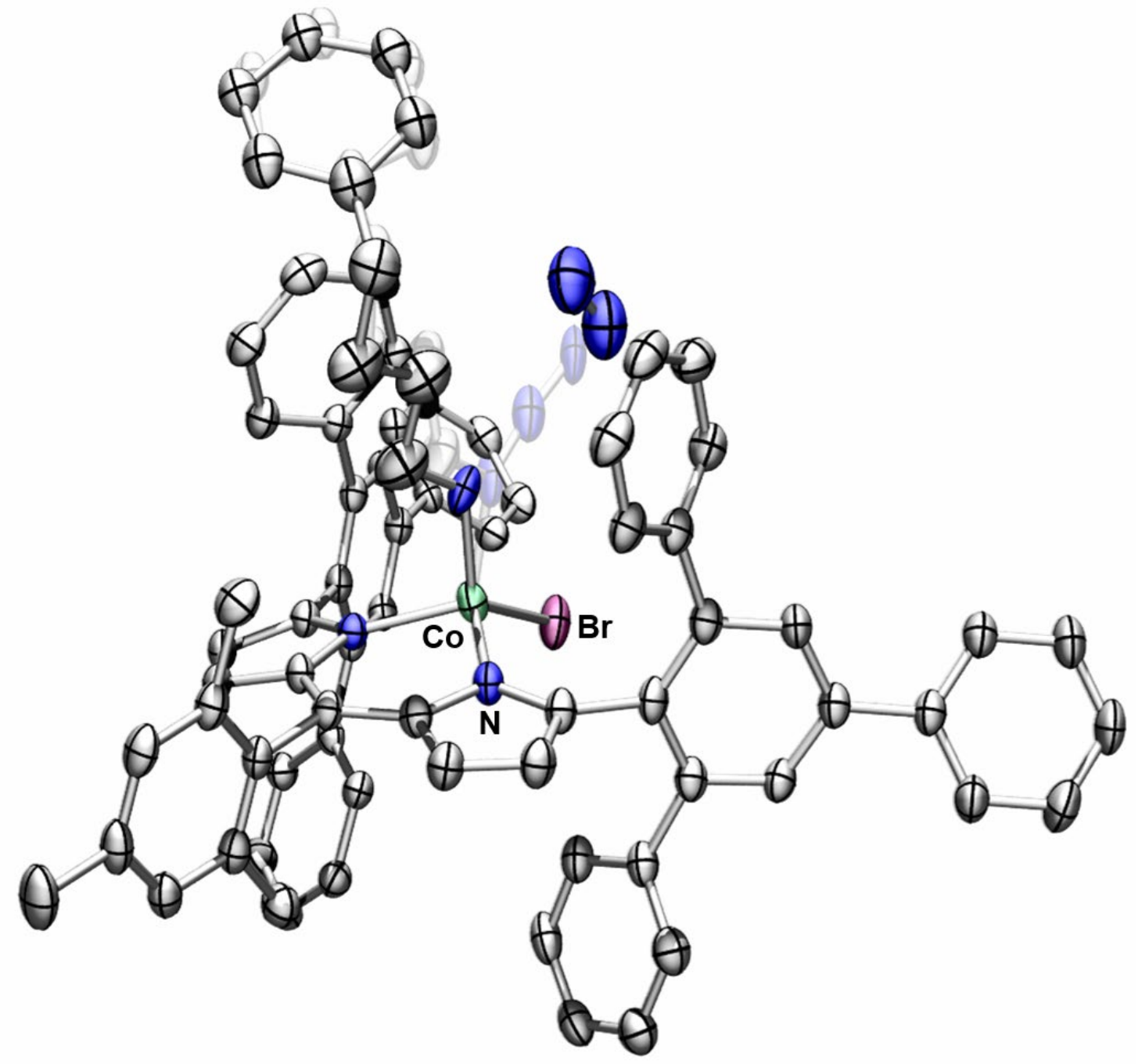

Figure S-32. Solid-state molecular structure for $\mathbf{7}-\mathbf{N}_{\mathbf{2}}$. Thermal ellipsoid plot showing the dark structure (faded) and the irradiated structure (bold) generated from complex 7. 


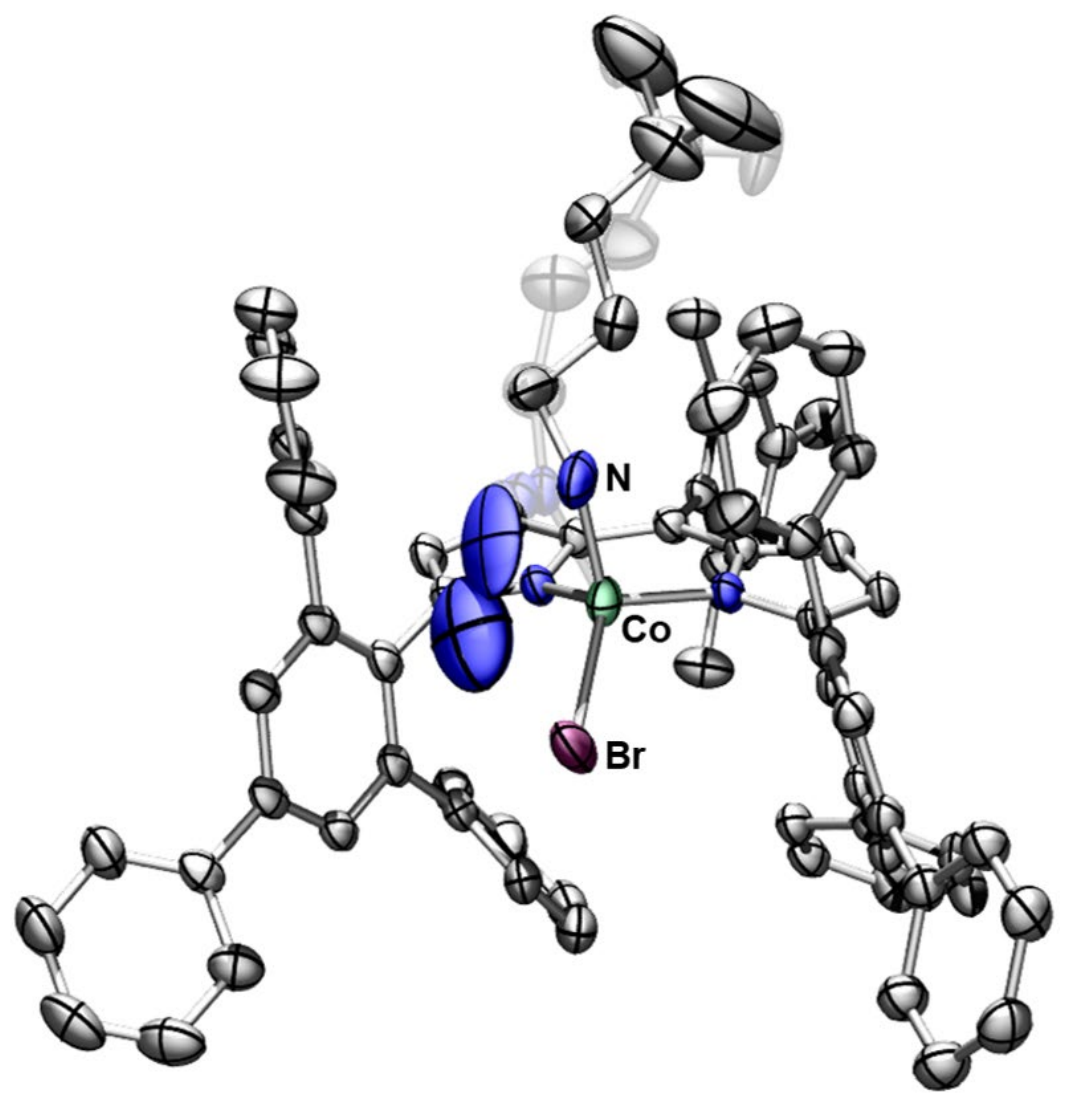

Figure S-33. Solid-state molecular structure for $\mathbf{8}-\mathbf{N}_{\mathbf{2}}$. Thermal ellipsoid plot showing the dark structure (faded) and the irradiated structure (bold) generated from complex $\mathbf{8}$. 
Table S-3. X-ray diffraction experimental details ${ }^{\mathrm{a}, \mathrm{b}}$

\begin{tabular}{|c|c|c|c|}
\hline & $\begin{array}{c}\left({ }^{\mathrm{Ar}} \mathrm{L}\right) \operatorname{CoBr}\left(\mathrm{N}_{3}\left(\mathrm{C}_{6} \mathrm{H}_{4}-p-{ }^{-} \mathrm{Bu}\right)\right) \\
(\mathbf{3})\end{array}$ & $\underset{(4)}{\left({ }^{A} \mathrm{~L}\right) \operatorname{CoBr}([3+2] \text { annulation })}$ & $\underset{(5)}{\left({ }^{\mathrm{A} r} \mathbf{L}\right) \operatorname{CoBr}\left(\mathrm{NH}_{2}\left(\mathrm{C}_{6} \mathrm{H}_{4}-p-{ }^{t} \mathrm{Bu}\right)\right)}$ \\
\hline $\begin{array}{l}\text { Moiety } \\
\text { Formula }\end{array}$ & $\mathrm{C}_{76} \mathrm{H}_{62} \mathrm{BrCoN}_{5} \cdot 2\left(\mathrm{C}_{7} \mathrm{H}_{8}\right)$ & $\mathrm{C}_{76} \mathrm{H}_{62} \mathrm{BrCoN}_{3} \cdot 3.5\left(\mathrm{C}_{6} \mathrm{H}_{6}\right)$ & $\mathrm{C}_{76} \mathrm{H}_{64} \mathrm{BrCoN}_{3} \cdot 2\left(\mathrm{C}_{6} \mathrm{H}_{6}\right)$ \\
\hline FW & 1368.41 & 1429.50 & 1314.35 \\
\hline $\begin{array}{l}\text { Crystal } \\
\text { System } \\
\text { Space }\end{array}$ & Monoclinic & Triclinic & Monoclinic \\
\hline $\begin{array}{l}\text { Group } \\
\text { (Z) }\end{array}$ & $P 2{ }_{1} / \mathrm{n}(4)$ & $P \overline{1}(2)$ & $P 2_{1} / \mathrm{c}(4)$ \\
\hline a $(\AA)$ & $11.5371(4)$ & $12.5766(4)$ & $17.7144(19)$ \\
\hline b $(\AA)$ & $22.8606(10)$ & $13.7287(5)$ & $21.629(2)$ \\
\hline c $(\AA)$ & $27.3251(12)$ & $22.5774(8)$ & $18.715(2)$ \\
\hline$\alpha\left(^{\circ}\right)$ & 90 & $100.712(1)$ & 90 \\
\hline$\beta\left(\left(^{\circ}\right)\right.$ & $94.279(4)$ & $94.999(1)$ & $108.264(2)$ \\
\hline$\gamma\left({ }^{\circ}\right)$ & 90 & $93.362(1)$ & 90 \\
\hline $\begin{array}{l}\text { Volume } \\
\left(\AA^{3}\right)\end{array}$ & $7186.8(5)$ & $3804.6(2)$ & $6809.3(13)$ \\
\hline $\begin{array}{l}\text { Calc. } \rho \\
\left(\mathrm{mg} / \mathrm{m}^{3}\right)\end{array}$ & 1.265 & 1.248 & 1.282 \\
\hline$\mu\left(\mathrm{mm}^{-1}\right)$ & 0.846 & 0.801 & 0.889 \\
\hline $\begin{array}{c}\text { Crystal } \\
\text { Size } \\
(\mathbf{m m})\end{array}$ & $0.20 \times 0.10 \times 0.06$ & $0.35 \times 0.20 \times 0.08$ & $0.40 \times 0.20 \times 0.15$ \\
\hline $\begin{array}{c}\text { Reflectio } \\
\text { ns }\end{array}$ & 12706 & 13508 & 12048 \\
\hline $\begin{array}{c}\text { Complet } \\
\text { eness (to } \\
2 \theta \text { ) }\end{array}$ & $\begin{array}{c}100 \% \\
25.027^{\circ}\end{array}$ & $\begin{array}{c}99.6 \% \\
25.085^{\circ}\end{array}$ & $\begin{array}{c}99.9^{\circ} \% \\
25.043^{\circ}\end{array}$ \\
\hline $\begin{array}{l}\text { GOF on } \\
F^{2}\end{array}$ & 1.083 & 1.014 & 1.029 \\
\hline 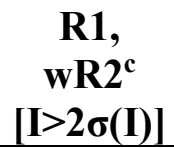 & $0.0872,0.1685$ & $0.0552,0.1230$ & $0.0451,0.1163$ \\
\hline
\end{tabular}

${ }^{\mathrm{a}} \lambda=0.71073 \AA ;{ }^{\mathrm{b}} \mathrm{T}=100(2) \mathrm{K} ;{ }^{\mathrm{c}} \mathrm{R} 1=\Sigma|| F_{o}|-| F_{\mathrm{c}}|| \Sigma\left|F_{o}\right|, \mathrm{wR} 2=\left\{\Sigma\left[w\left(F_{o}{ }^{2}-F_{c}{ }^{2}\right)^{2}\right] / \Sigma\left[w\left(F_{o}{ }^{2}\right)^{2}\right]\right\}^{1 / 2}$ 


\begin{tabular}{|c|c|c|c|}
\hline & $\begin{array}{c}\left({ }^{\mathrm{Ar}} \mathrm{L}\right) \mathrm{CoBr}\left(\mathrm{N}_{3} \mathrm{R}\right) \\
\mathrm{R}=\underset{(\mathrm{C})}{\left(\mathrm{CH}_{2}\right)_{4} \mathrm{Ph}} \\
(7)\end{array}$ & $\begin{array}{c}\left({ }^{\mathrm{Ar}} \mathrm{L}\right) \mathrm{CoBr}\left(\mathrm{N}_{3} \mathrm{R}\right) \\
\mathrm{R}=\underset{(\mathbf{C})}{\left(\mathrm{CH}_{2}\right)_{3} \mathrm{C}\left(\mathrm{CH}_{3}\right)}\end{array}$ & $\begin{array}{c}\left({ }^{\mathrm{Ar}} \mathrm{L}\right) \mathrm{CoBr}\left(\mathrm{NHCHC}_{6} \mathrm{H}_{5}\right) \\
(9)\end{array}$ \\
\hline $\begin{array}{l}\text { Moiety } \\
\text { Formula }\end{array}$ & $\mathrm{C}_{76} \mathrm{H}_{62} \mathrm{BrCoN}_{5}$ & $\mathrm{C}_{72} \mathrm{H}_{62} \mathrm{BrCoN}_{5} \cdot 0.5\left(\mathrm{C}_{6} \mathrm{H}_{14}\right)$ & $2\left(\mathrm{C}_{73} \mathrm{H}_{56} \mathrm{BrCoN}_{3}\right) \cdot 3\left(\mathrm{C}_{7} \mathrm{H}_{8}\right)$ \\
\hline FW & 1184.14 & 1179.19 & 1252.25 \\
\hline $\begin{array}{l}\text { Crystal } \\
\text { System } \\
\text { Space }\end{array}$ & Triclinic & Monoclinic & Triclinic \\
\hline $\begin{array}{c}\text { Group } \\
\text { (Z) }\end{array}$ & $P \overline{1}(2)$ & $P 2_{1} / \mathrm{c}(4)$ & $P \overline{1}(2)$ \\
\hline a $(\AA)$ & $11.8481(4)$ & $11.5036(5)$ & $11.7393(6)$ \\
\hline b $(\AA)$ & $12.2353(3)$ & $21.4756(11)$ & $14.6478(7)$ \\
\hline c $(\AA)$ & $22.6467(7)$ & $25.3211(13)$ & $19.8506(10)$ \\
\hline$\alpha\left(^{\circ}\right)$ & $95.171(2)$ & 90 & $81.004(1)$ \\
\hline$\beta\left(^{\circ}\right)$ & $101.347(3)$ & $99.4205(16)$ & $80.061(1)$ \\
\hline$\gamma\left({ }^{\circ}\right)$ & $107.834(3)$ & 90 & $75.351(1)$ \\
\hline $\begin{array}{l}\text { Volume } \\
\qquad\left(\AA^{3}\right)\end{array}$ & $3027.11(17)$ & $6171.1(5)$ & $3230.1(3)$ \\
\hline $\begin{array}{c}\text { Calc. } \rho \\
\left(\mathrm{mg} / \mathrm{m}^{3}\right)\end{array}$ & 1.300 & 1.269 & 1.288 \\
\hline$\mu\left(\mathrm{mm}^{-1}\right)$ & 0.993 & 0.973 & 0.933 \\
\hline $\begin{array}{l}\text { Crystal } \\
\text { Size } \\
(\mathbf{m m})\end{array}$ & $0.25 \times 0.20 \times 0.05$ & $0.39 \times 0.25 \times 0.16$ & $0.18 \times 0.10 \times 0.06$ \\
\hline $\begin{array}{c}\text { Reflectio } \\
\text { ns }\end{array}$ & 10711 & 10995 & 11459 \\
\hline $\begin{array}{l}\text { Complet } \\
\text { eness (to } \\
2 \theta)\end{array}$ & $\begin{array}{c}99.8 \% \\
25.058^{\circ}\end{array}$ & $\begin{array}{c}99.4 \% \\
25.096^{\circ}\end{array}$ & $\begin{array}{l}99.9 \% \\
25.061^{\circ}\end{array}$ \\
\hline $\begin{array}{l}\text { GOF on } \\
\text { F }^{2}\end{array}$ & 1.011 & 1.016 & 1.006 \\
\hline $\begin{array}{c}\text { R1, } \\
\text { wR2 }^{\mathbf{c}} \\
{[\mathbf{I}>\mathbf{2 \sigma}(\mathbf{I})]}\end{array}$ & $0.0495,0.1045$ & $0.0605,0.1713$ & $0.0395,0.0835$ \\
\hline
\end{tabular}




\begin{tabular}{|c|c|c|c|c|}
\hline & $\begin{array}{c}{\left[\left(^{\mathrm{Bu}} \mathrm{L}\right) \mathrm{CoBr}\right]_{2}} \\
(\mathbf{1 1})\end{array}$ & $\begin{array}{c}\left({ }^{\mathrm{Bu} u} \mathrm{~L}\right) \mathrm{CoBr}(\mathrm{thf}) \\
(12)\end{array}$ & $\begin{array}{c}\left({ }^{(\mathrm{Bu} u} \mathrm{L}\right) \mathrm{CoBr}(1,2,3- \\
\text { dihydrotriazole }) \\
(14-\mathrm{Br})\end{array}$ & $\begin{array}{c}\left({ }^{(\mathrm{Bu}} \mathrm{L}\right) \mathrm{CoBr}(2- \\
\text { phenylpyrrolidine }) \\
(16-\mathrm{Br})\end{array}$ \\
\hline $\begin{array}{c}\text { Moiety } \\
\text { Formula }\end{array}$ & $\mathrm{C}_{26} \mathrm{H}_{33} \mathrm{BrCoN}_{2}$ & $\begin{array}{c}\mathrm{C}_{30} \mathrm{H}_{41} \mathrm{BrCoN}_{2} \\
\mathrm{O}\end{array}$ & $\mathrm{C}_{32} \mathrm{H}_{44} \mathrm{BrCoN}_{5}$ & $\mathrm{C}_{36} \mathrm{H}_{46} \mathrm{BrCoN}_{3}$ \\
\hline FW & 512.38 & 1168.97 & 637.56 & 659.60 \\
\hline $\begin{array}{l}\text { Crystal } \\
\text { System }\end{array}$ & Triclinic & Triclinic & Monoclinic & Triclinic \\
\hline $\begin{array}{c}\text { Space } \\
\text { Group }(Z)\end{array}$ & $P \overline{1}(2)$ & $P \overline{1}(2)$ & $P 2_{1} / \mathrm{c}(4)$ & $P \overline{1}(2)$ \\
\hline $\mathbf{a}(\AA)$ & $9.8826(5)$ & $14.3907(10)$ & $10.5174(9)$ & $9.3829(7)$ \\
\hline b $(\AA)$ & $10.8049(4)$ & $14.9859(10)$ & $18.4889(16)$ & $13.2694(10)$ \\
\hline c $(\AA)$ & $13.3280(6)$ & $15.2440(11)$ & $17.0030(15)$ & $14.2996(11)$ \\
\hline$\alpha\left(^{\circ}\right)$ & $108.738(1)$ & $92.015(1)$ & 90 & $67.174(2)$ \\
\hline$\beta\left({ }^{\circ}\right)$ & $92.612(1)$ & $98.561(1)$ & $105.908(2)$ & $84.707(2)$ \\
\hline$\gamma\left({ }^{\circ}\right)$ & $112.333(1)$ & $115.785(1)$ & 90 & $82.391(2)$ \\
\hline $\begin{array}{c}\text { Volume } \\
\qquad\left(\AA^{3}\right)\end{array}$ & $1223.60(10)$ & 2908.7(4) & $3179.7(5)$ & $1624.9(2)$ \\
\hline $\begin{array}{c}\text { Calc. } \rho \\
\left(\mathrm{mg} / \mathrm{m}^{3}\right)\end{array}$ & 1.391 & 1.335 & 1.332 & 1.348 \\
\hline$\mu\left(\mathrm{mm}^{-1}\right)$ & 2.349 & 1.987 & 1.825 & 1.786 \\
\hline $\begin{array}{c}\text { Crystal } \\
\text { Size }(\mathbf{m m})\end{array}$ & $\begin{array}{c}0.25 \times 0.20 \times 0.1 \\
5\end{array}$ & $\begin{array}{c}0.40 \times 0.20 \times 0.1 \\
8\end{array}$ & $0.30 \times 0.19 \times 0.07$ & $0.20 \times 0.18 \times 0.06$ \\
\hline Reflections & 4306 & 10281 & 5637 & 5746 \\
\hline $\begin{array}{l}\text { Completen } \\
\text { ess (to 20) }\end{array}$ & $\begin{array}{l}99.2 \% \\
25.072^{\circ}\end{array}$ & $\begin{array}{c}98.9 \% \\
25.048^{\circ}\end{array}$ & $\begin{array}{c}100 \% \\
25.045^{\circ}\end{array}$ & $\begin{array}{c}99.6 \% \\
25.077^{\circ}\end{array}$ \\
\hline GOF on $\mathrm{F}^{2}$ & 1.028 & 1.062 & 1.058 & 1.015 \\
\hline $\begin{array}{l}\text { R1, wR2 } \\
{[\mathrm{I}>\mathbf{2 \sigma}(\mathrm{I})]}\end{array}$ & $0.0351,0.0725$ & $0.0269,0.0607$ & $0.0352,0.0852$ & $0.0546,0.1212$ \\
\hline
\end{tabular}




\begin{tabular}{|c|c|c|c|}
\hline & $\begin{array}{c}\left({ }^{\mathrm{Bu}} \mathrm{L}\right) \mathrm{CoCl}(2,2- \\
\text { dimehtylpyrrolidine }) \\
(18)\end{array}$ & $\left({ }^{t \mathrm{~B} u} \mathrm{~L}\right) \mathrm{CoCl}\left(\mathrm{NHCH}\left(\mathrm{CH}_{2}\right)_{2} \mathrm{CH}_{3}\right)$ & $\begin{array}{c}\left({ }^{\mathrm{Ar}} \mathrm{L}\right) \mathrm{CoCl}\left(\mathrm{NH}_{2}\left(\mathrm{CH}_{2}\right)_{3} \mathrm{CH}_{3}\right) \\
(21)\end{array}$ \\
\hline $\begin{array}{l}\text { Moiety } \\
\text { Formula }\end{array}$ & $2\left(\mathrm{C}_{16} \mathrm{H}_{23} \mathrm{Cl}_{0.5} \mathrm{Co}_{0.5} \mathrm{~N}_{1.5}\right)$ & $\mathrm{C}_{30} \mathrm{H}_{42} \mathrm{ClCoN}_{3}$ & $\mathrm{C}_{30} \mathrm{H}_{44} \mathrm{ClCoN}_{3}$ \\
\hline FW & 567.10 & 539.04 & 541.06 \\
\hline $\begin{array}{l}\text { Crystal } \\
\text { System } \\
\text { Space }\end{array}$ & Orthorhombic & Monoclinic & Monoclinic \\
\hline $\begin{array}{l}\text { Group } \\
\text { (Z) }\end{array}$ & Pnma (4) & $P 2_{1} / \mathrm{n}(4)$ & $P 2_{1} / \mathrm{n}(4)$ \\
\hline a $(\AA)$ & $19.6732(14)$ & $13.892(2)$ & $13.8661(8)$ \\
\hline b $(\AA)$ & $8.7859(8)$ & $13.8768(18)$ & $13.8215(9)$ \\
\hline c $(\AA)$ & $17.5128(12)$ & $16.289(2)$ & $16.3606(10)$ \\
\hline$\alpha\left(^{\circ}\right)$ & 90 & 90 & 90 \\
\hline$\beta\left({ }^{\circ}\right)$ & 90 & $111.131(2)$ & $111.490(2)$ \\
\hline$\gamma\left({ }^{\circ}\right)$ & 90 & 90 & 90 \\
\hline $\begin{array}{l}\text { Volume } \\
\qquad\left(\AA^{3}\right)\end{array}$ & $3027.0(4)$ & $2928.9(7)$ & 2917.5(3) \\
\hline $\begin{array}{c}\text { Calc. } \rho \\
\left(\mathbf{m g} / \mathbf{m}^{3}\right)\end{array}$ & 1.244 & 1.222 & 1.232 \\
\hline$\mu\left(\mathrm{mm}^{-1}\right)$ & 0.680 & 0.699 & 0.703 \\
\hline $\begin{array}{l}\text { Crystal } \\
\text { Size } \\
(\mathbf{m m})\end{array}$ & $0.20 \times 0.15 \times 0.13$ & $0.40 \times 0.18 \times 0.09$ & $0.50 \times 0.30 \times 0.10$ \\
\hline $\begin{array}{c}\text { Reflectio } \\
\text { ns }\end{array}$ & 2879 & 5133 & 5194 \\
\hline $\begin{array}{c}\text { Complet } \\
\text { eness (to } \\
2 \theta)\end{array}$ & $\begin{array}{l}99.7 \% \\
25.085^{\circ}\end{array}$ & $\begin{array}{l}98.8 \% \\
25.052^{\circ}\end{array}$ & $\begin{array}{l}99.7 \% \\
25.100^{\circ}\end{array}$ \\
\hline $\begin{array}{c}\text { GOF on } \\
F^{2}\end{array}$ & 1.181 & 1.001 & 1.084 \\
\hline $\begin{array}{c}\text { R1, } \\
\text { WR2 }^{\mathbf{c}} \\
{[\mathbf{I}>\mathbf{2 \sigma}(\mathbf{I})]}\end{array}$ & $0.0685,0.1450$ & $0.0581,0.1289$ & $0.0445,0.0813$ \\
\hline
\end{tabular}




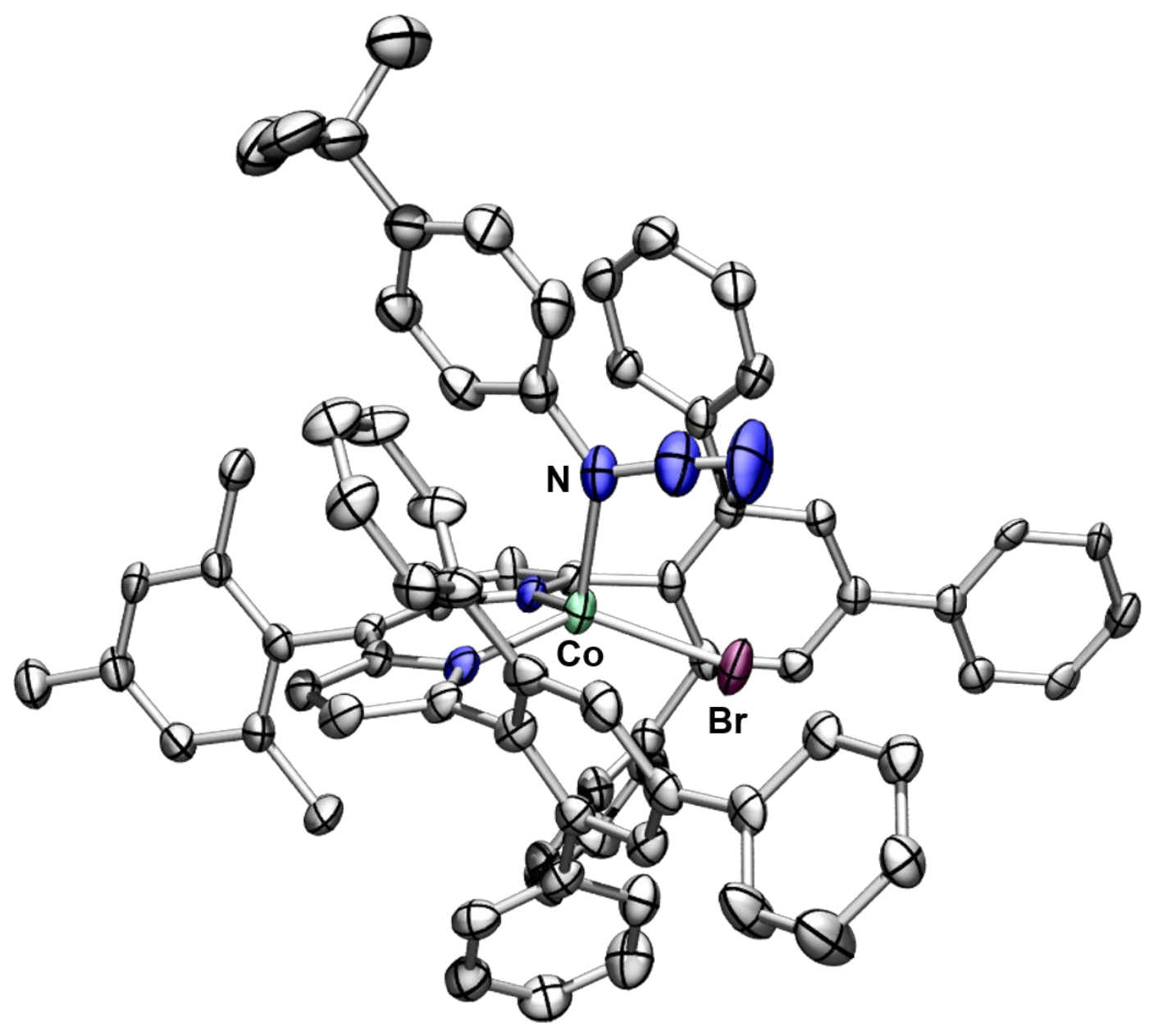

Figure S-34. Solid-state molecular structure for $\left({ }^{\mathrm{Ar}} \mathrm{L}\right) \mathrm{CoBr}\left(\mathrm{N}_{3}\left(\mathrm{C}_{6} \mathrm{H}_{4}-p-{ }^{t} \mathrm{Bu}\right)\right)$ (3) with thermal ellipsoids at 50\% probability level. Hydrogens omitted for clarity. 


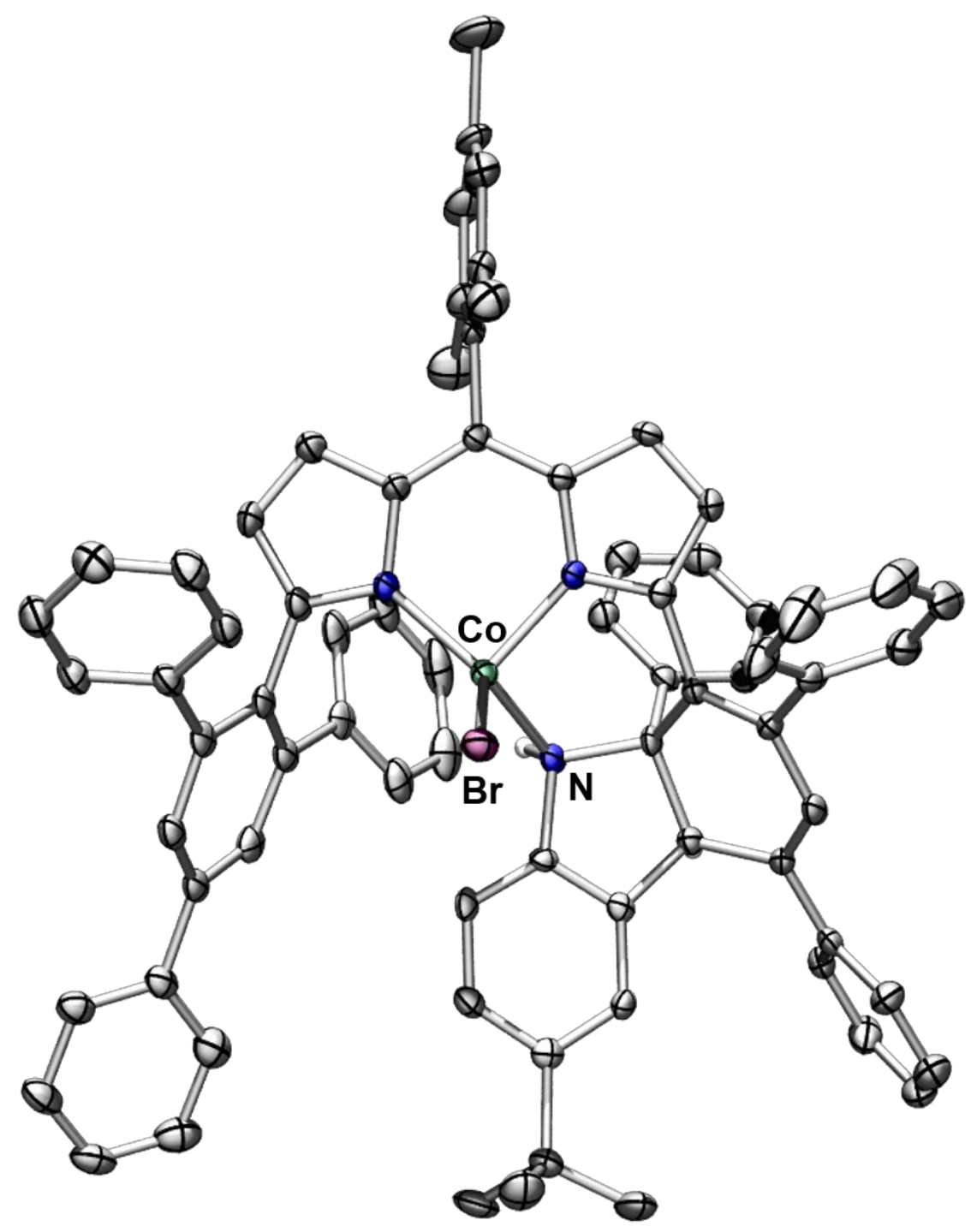

Figure S-35. Solid-state molecular structure for $\left({ }^{\mathrm{Ar}} \mathrm{L}\right) \operatorname{CoBr}([3+2]$ annulation) (4) with thermal ellipsoids at $50 \%$ probability level. Hydrogens omitted for clarity, except for $\mathrm{N}-H$. 


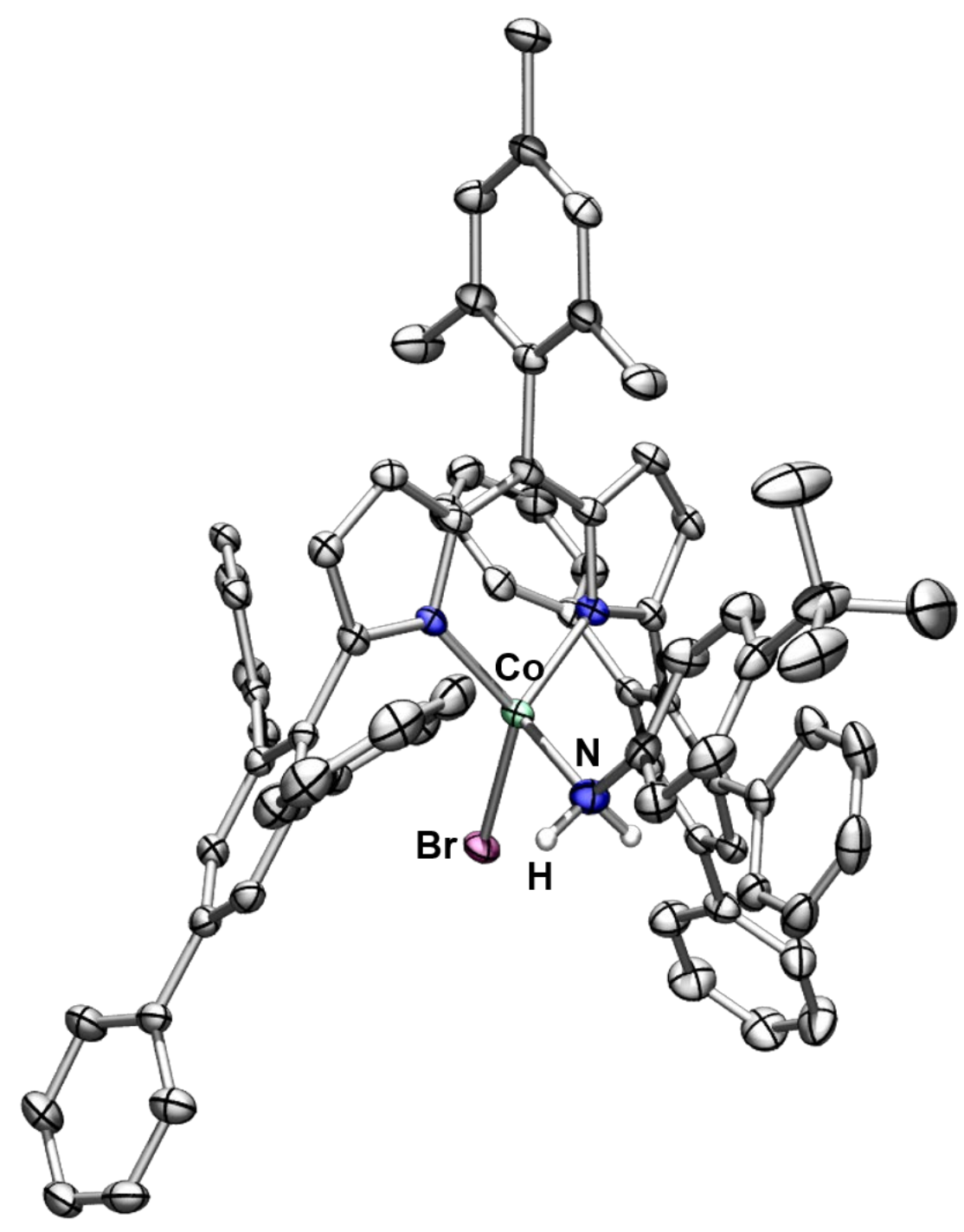

Figure S-36. Solid-state molecular structure for $\left({ }^{\mathrm{Ar}} \mathrm{L}\right) \mathrm{CoBr}\left(\mathrm{NH}_{2}\left(\mathrm{C}_{6} \mathrm{H}_{4}-p-{ }^{t} \mathrm{Bu}\right)\right)(5)$ with thermal ellipsoids at 50\% probability level. Hydrogens omitted for clarity, except for aniline $\mathrm{NH}_{2}$. 


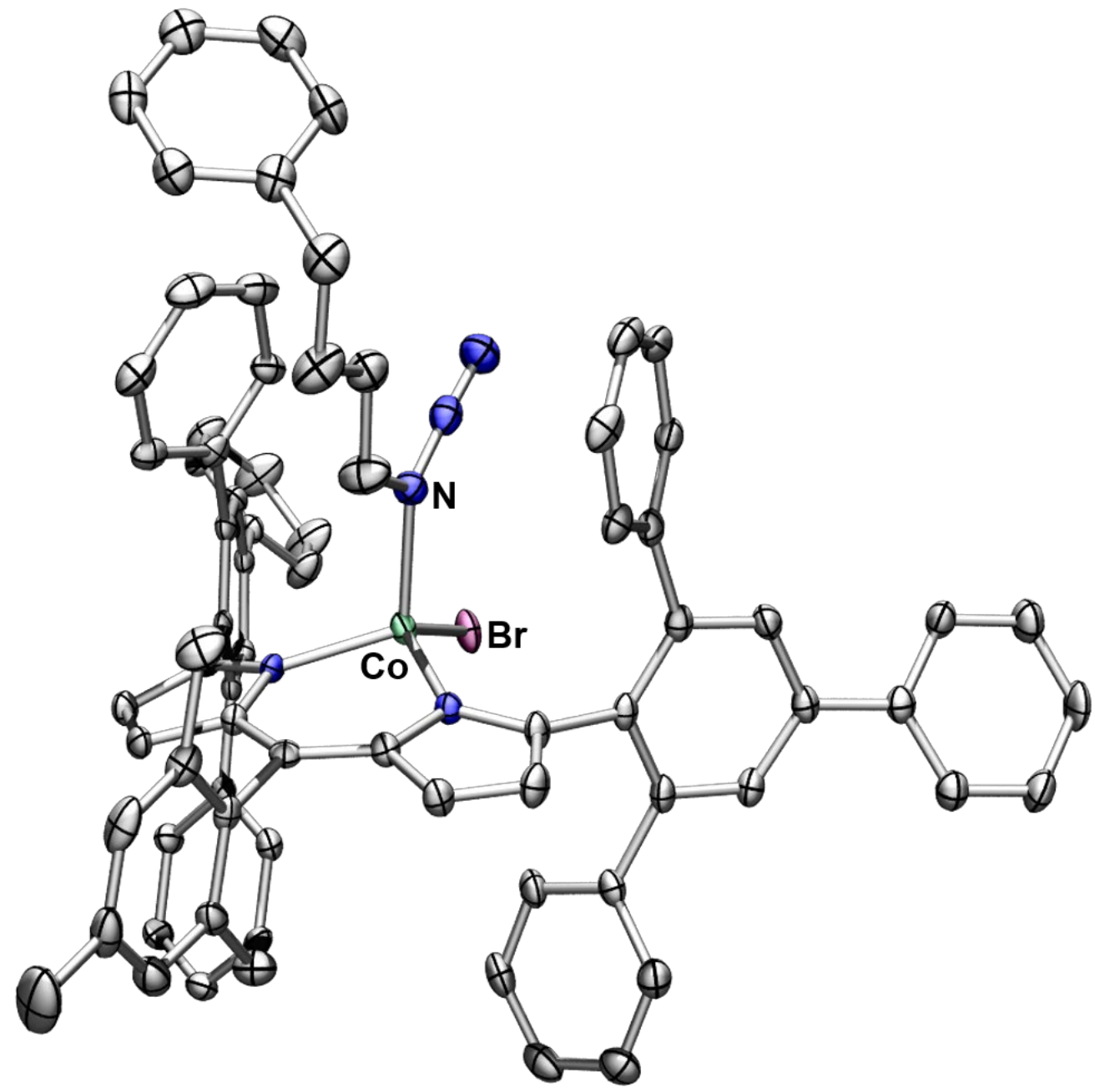

Figure S-37. Solid-state molecular structure for $\left({ }^{\mathrm{Ar}} \mathrm{L}\right) \mathrm{CoBr}\left(\mathrm{N}_{3} \mathrm{R}\right)$ (7) with thermal ellipsoids at $50 \%$ probability level. Hydrogens omitted for clarity. 


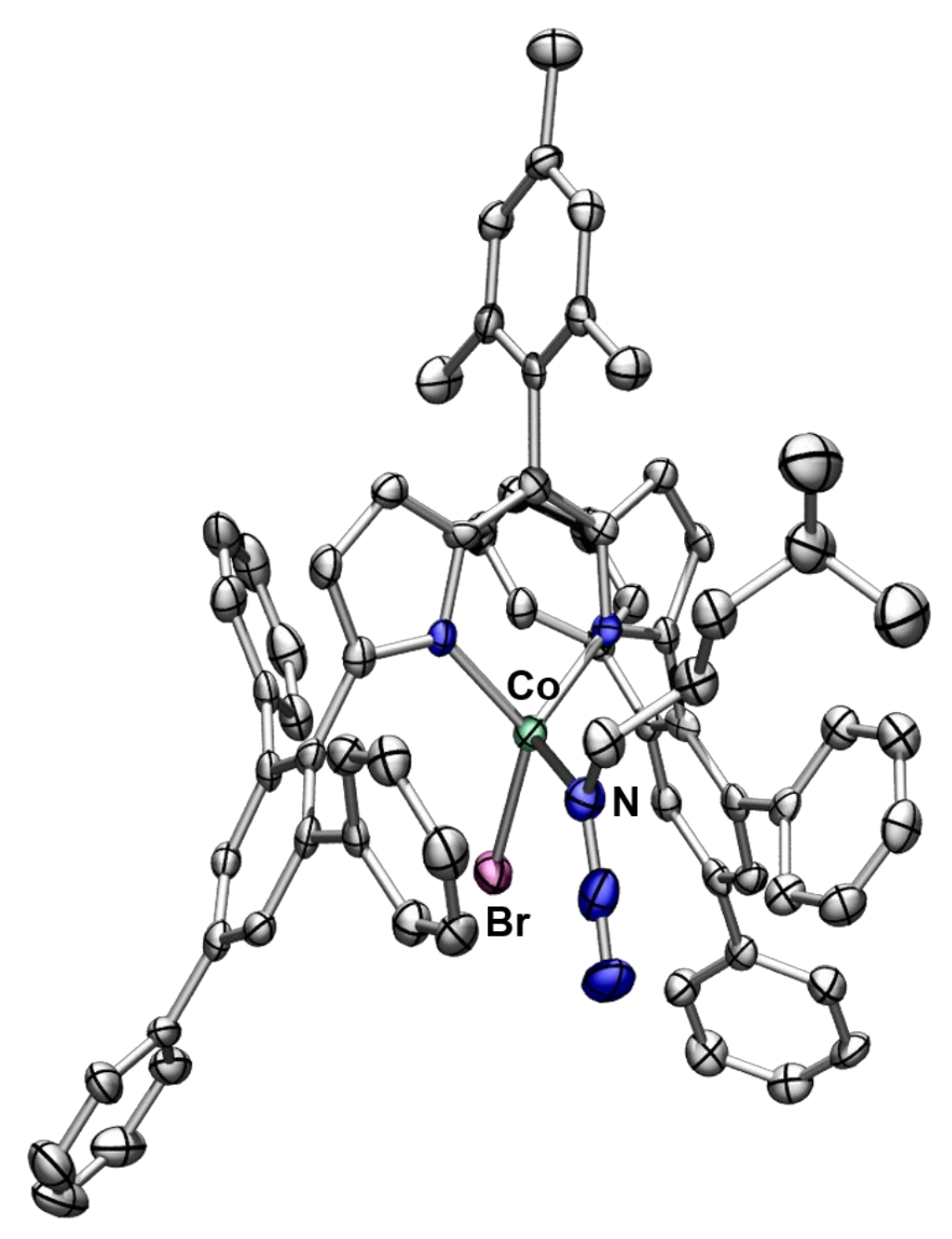

Figure S-38. Solid-state molecular structure for $\left({ }^{A r} L\right) \operatorname{CoBr}\left(N_{3} R\right)(8)$ with thermal ellipsoids at $50 \%$ probability level. Hydrogens omitted for clarity. 


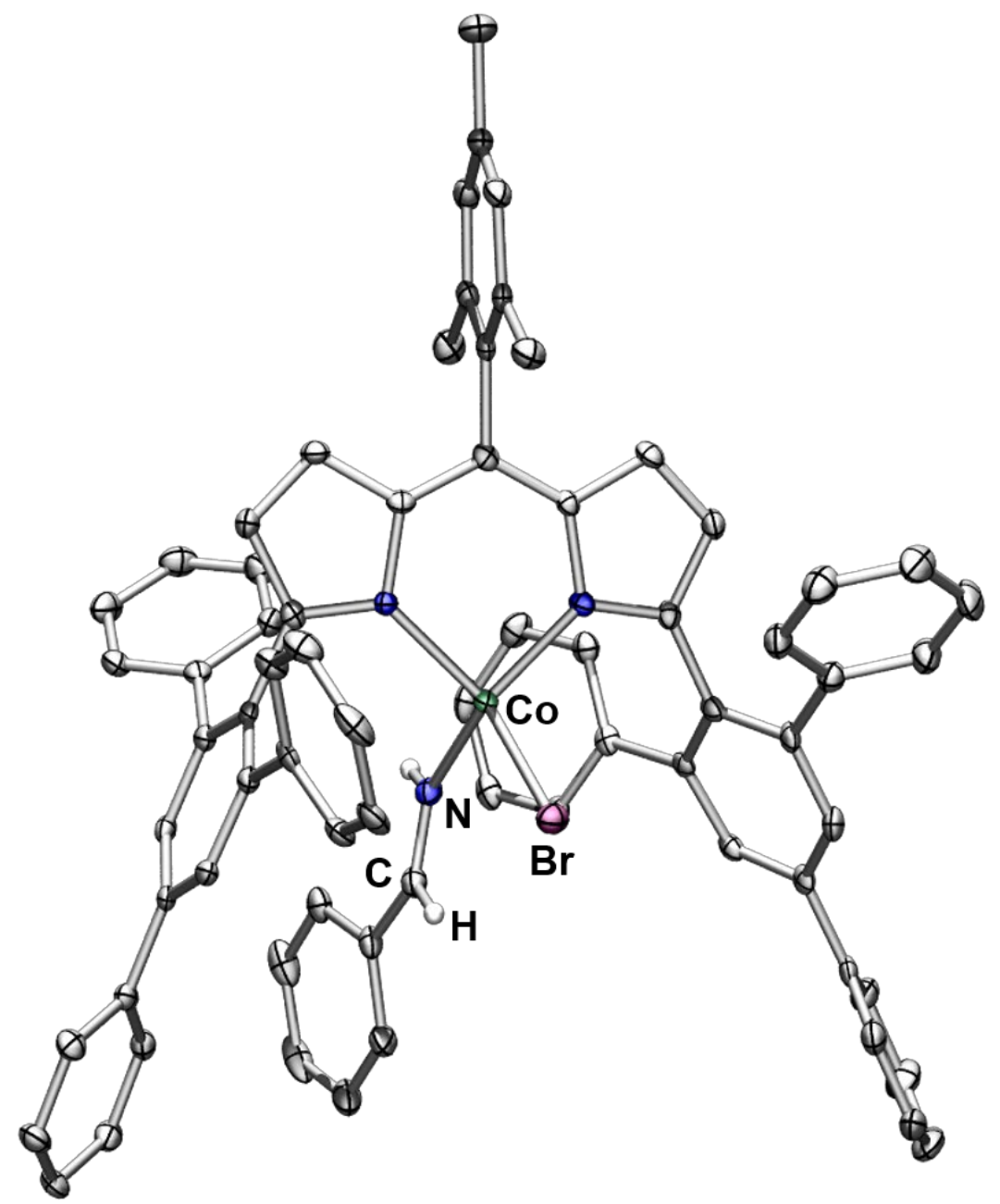

Figure S-39. Solid-state molecular structure for $\left({ }^{\mathrm{Ar}} \mathrm{L}\right) \mathrm{CoBr}\left(\mathrm{NHCHC}_{6} \mathrm{H}_{5}\right)$ (9) with thermal ellipsoids at 50\% probability level. Hydrogens omitted for clarity, except for the imine $H \mathrm{~N}=\mathrm{C} H \mathrm{Ph}$. 


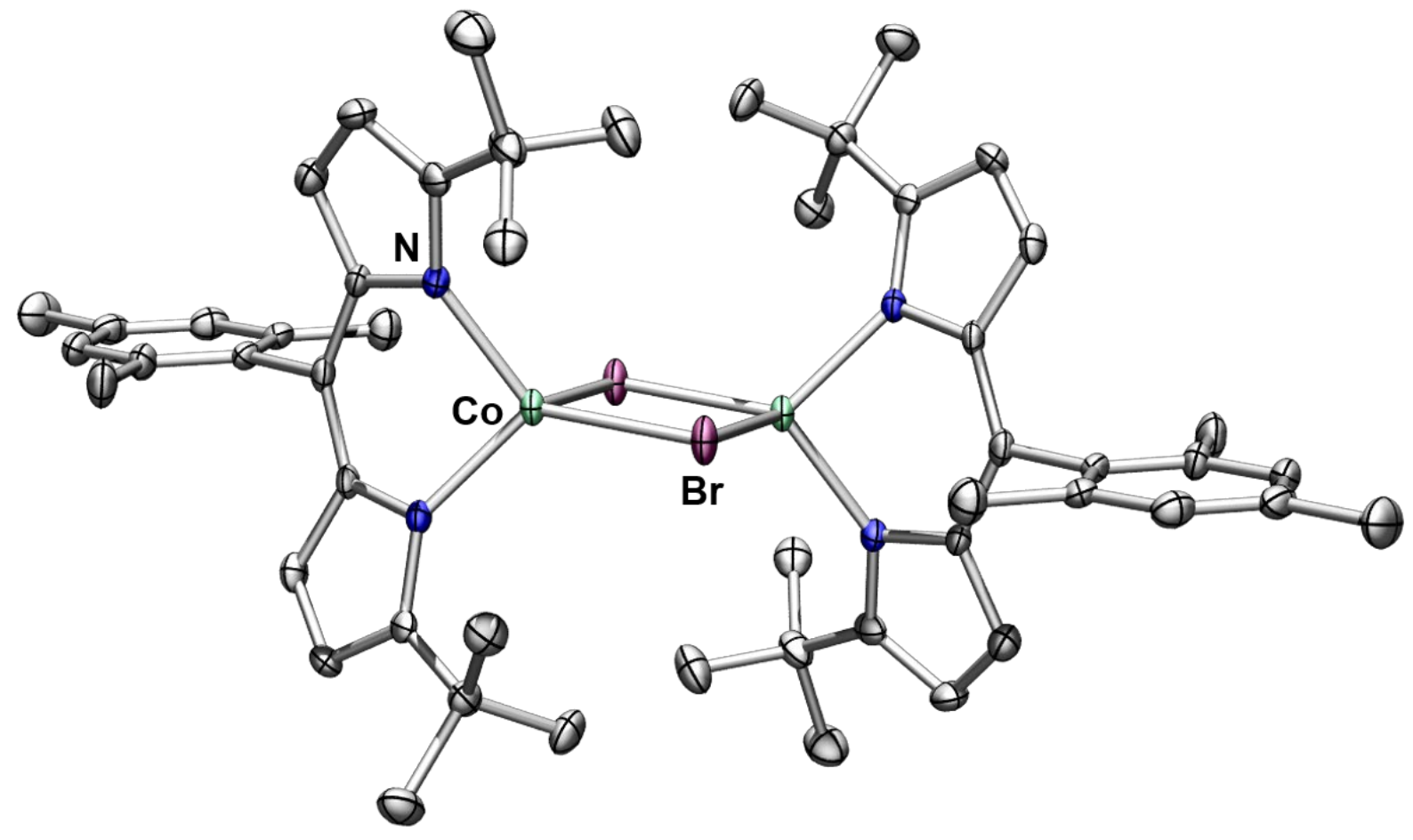

Figure S-40. Solid-state molecular structure for $\left[\left({ }^{t B u} \mathrm{~L}\right) \mathrm{CoBr}\right]_{2}(\mathbf{1 1})$ with thermal ellipsoids at $50 \%$ probability level. Hydrogens omitted for clarity. 


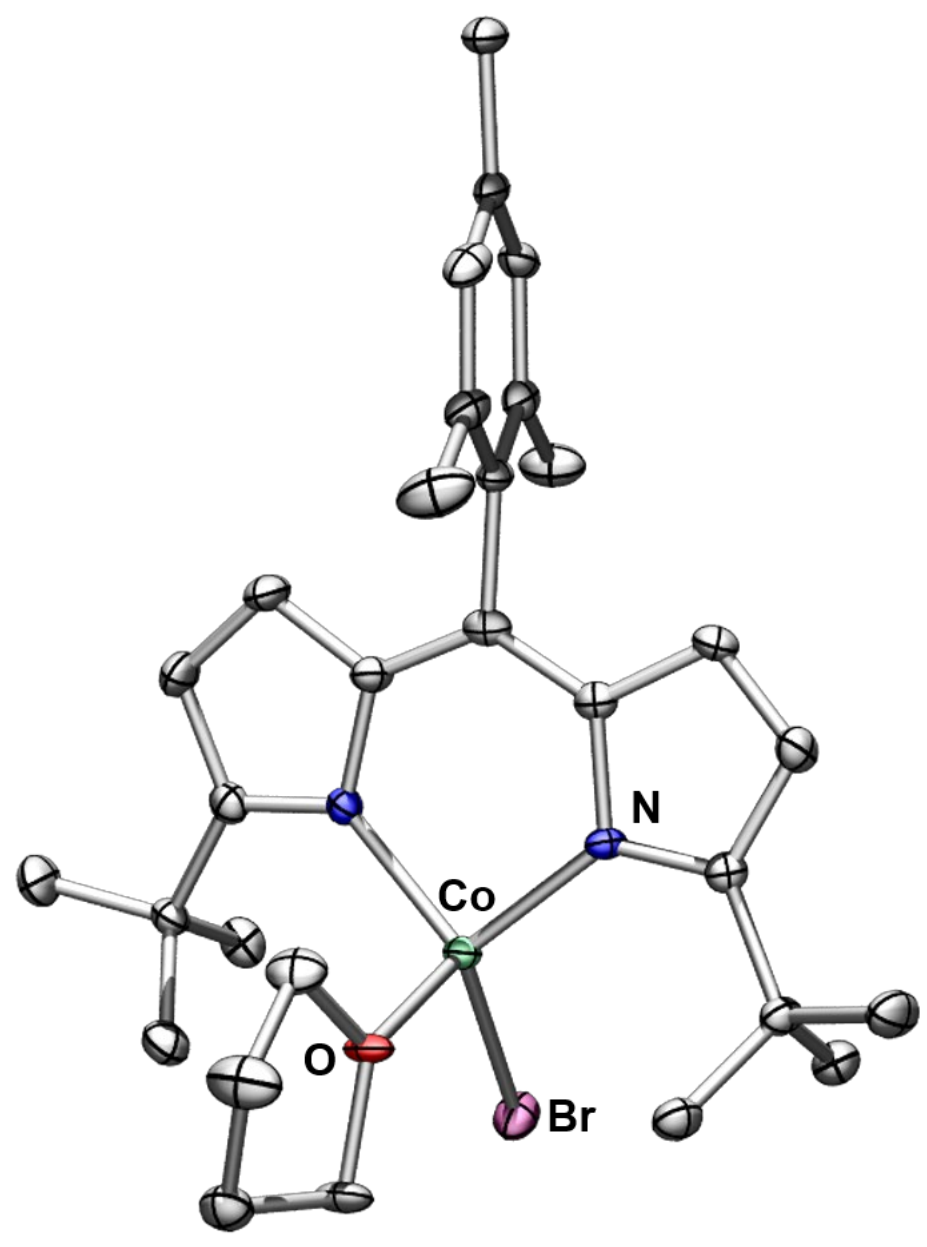

Figure S-41. Solid-state molecular structure for $\left({ }^{t B u} \mathrm{~L}\right) \operatorname{CoBr}(\operatorname{thf})(\mathbf{1 2})$ with thermal ellipsoids at $50 \%$ probability level. Hydrogens omitted for clarity. 


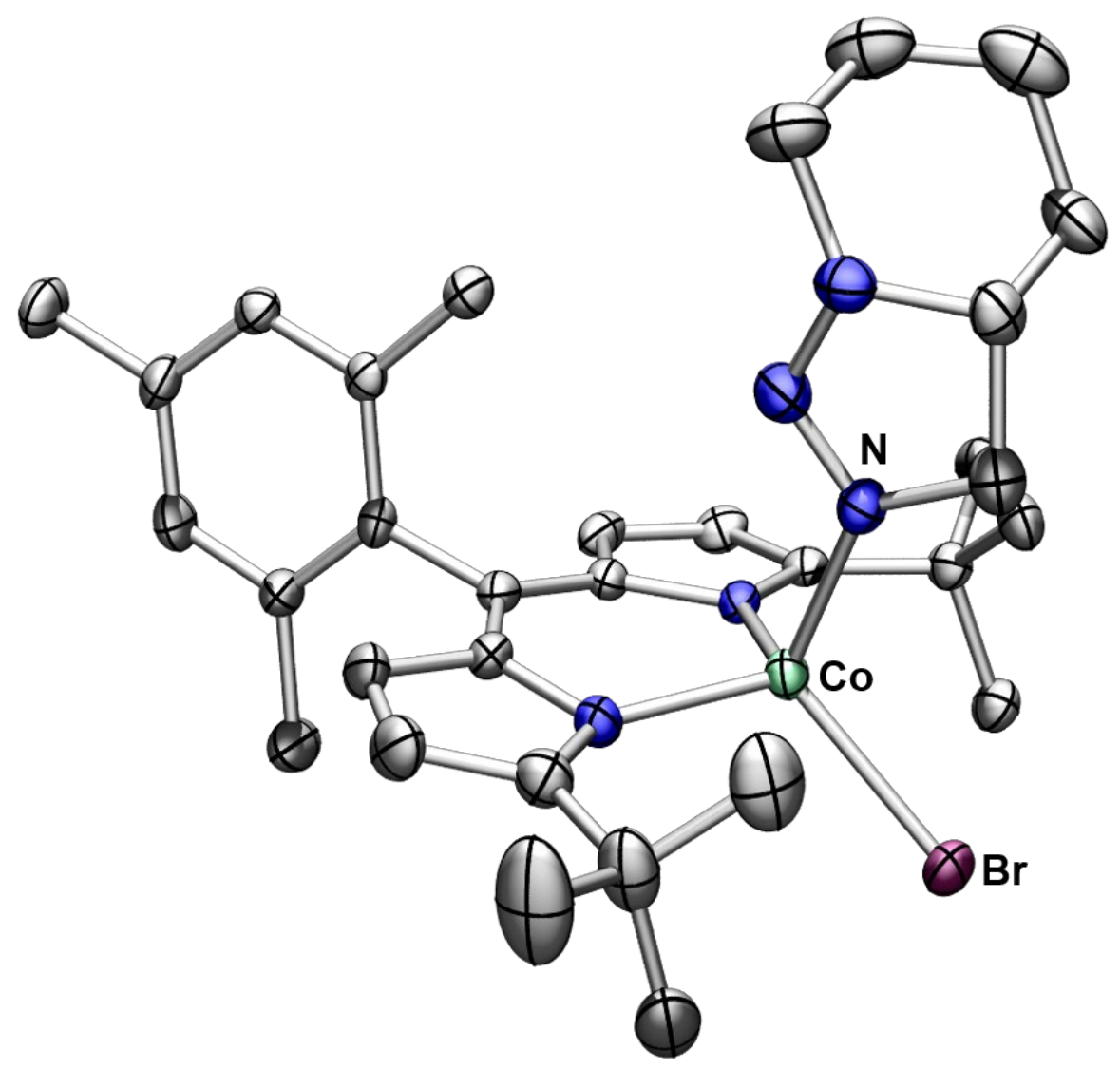

Figure S-42. Solid-state molecular structure for $\left({ }^{t B u} \mathrm{~L}\right) \operatorname{CoBr}(1,2,3$-dihydrotriazole) (14-Br) with thermal ellipsoids at 50\% probability level. Hydrogens omitted for clarity. 


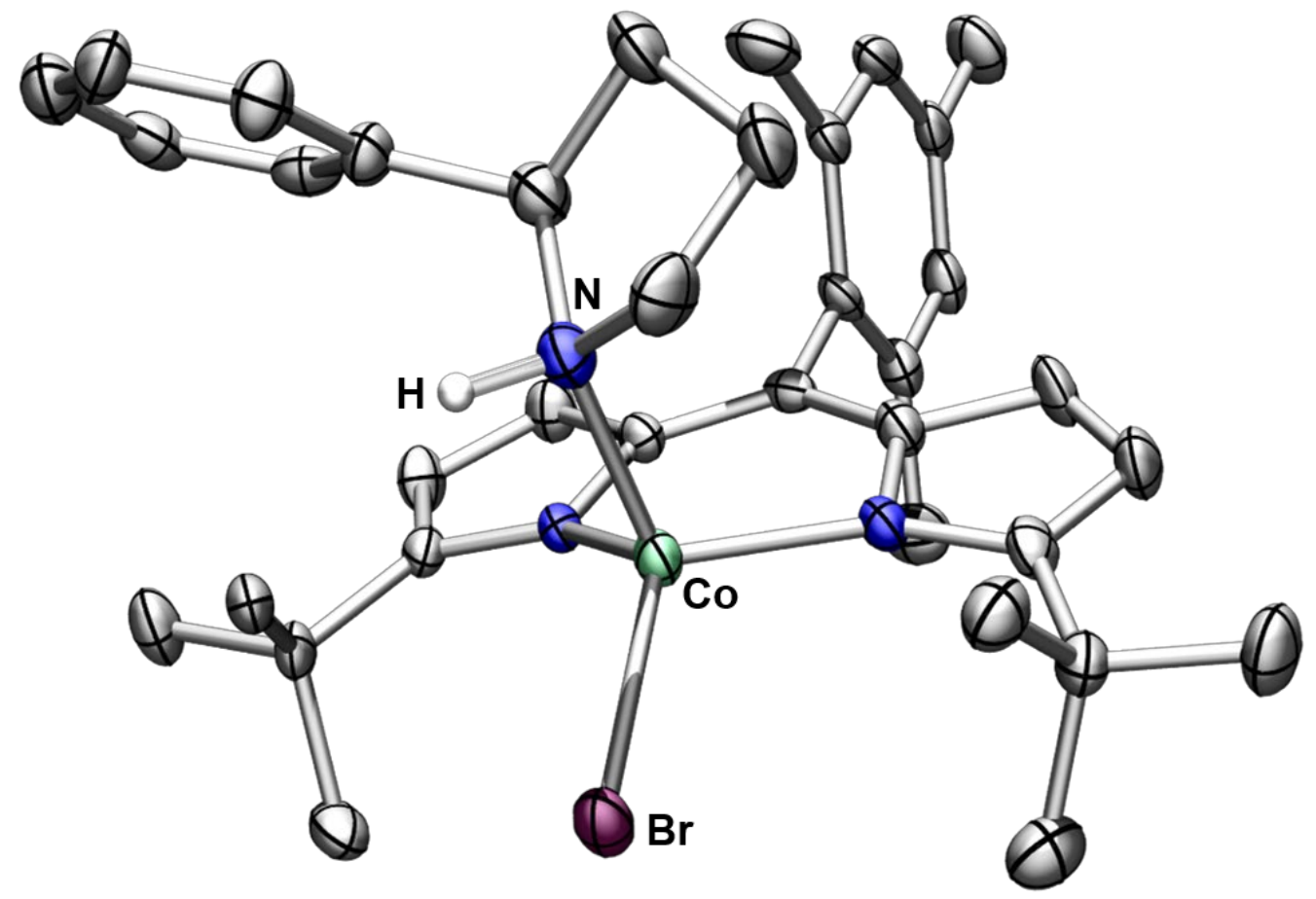

Figure S-43. Solid-state molecular structure for $\left({ }^{t \mathrm{Bu}} \mathrm{L}\right) \mathrm{CoBr}(2$-phenylpyrrolidine) (16-Br) with thermal ellipsoids at 50\% probability level. Hydrogens omitted for clarity, except for the pyrrolidine $\mathrm{N}-H$. 


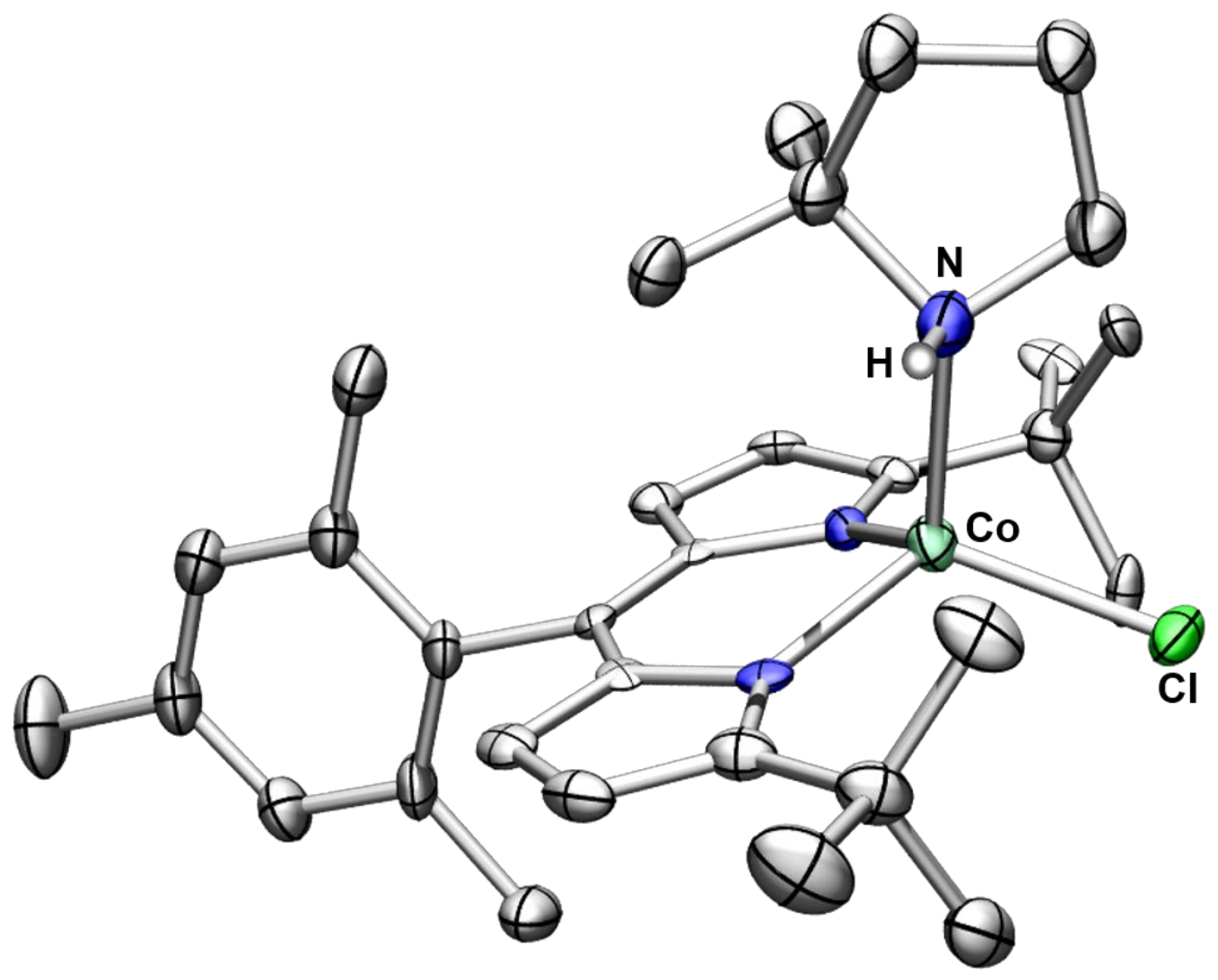

Figure S-44. Solid-state molecular structure for $\left({ }^{t \mathrm{Bu}} \mathrm{L}\right) \mathrm{CoCl}(2,2$-dimethylpyrrolidine) (18) with thermal ellipsoids at 50\% probability level. Hydrogens omitted for clarity, except for the pyrrolidine $\mathrm{N}-H$ 


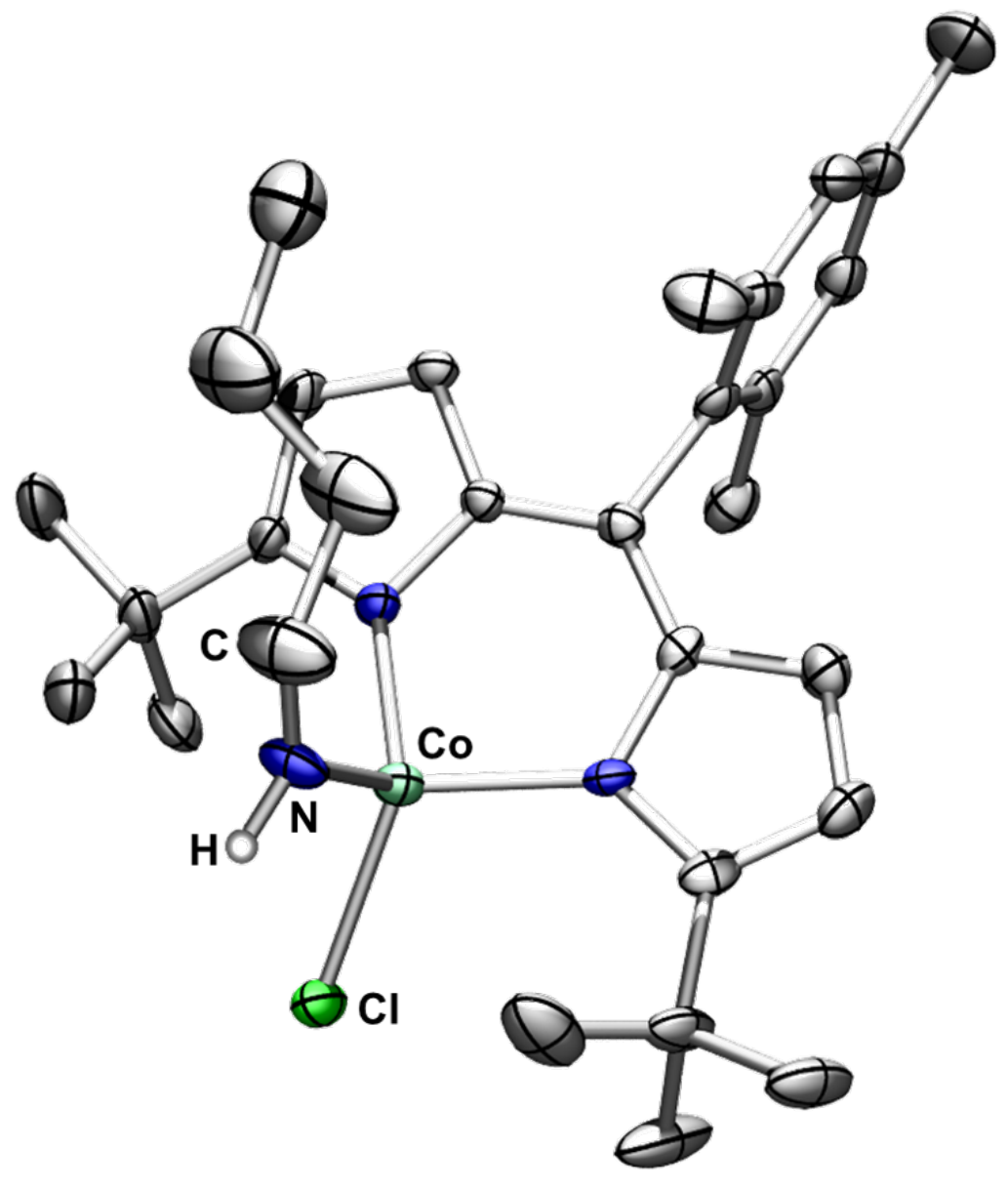

Figure S-45. Solid-state molecular structure for $\left({ }^{t \mathrm{Bu}} \mathrm{L}\right) \mathrm{CoCl}\left(\mathrm{NHCH}\left(\mathrm{CH}_{2}\right) \mathrm{CH}_{3}\right)(\mathbf{2 0})$ with thermal ellipsoids at 50\% probability level. Hydrogens omitted for clarity, except for the imine $\mathrm{N}-H$. 


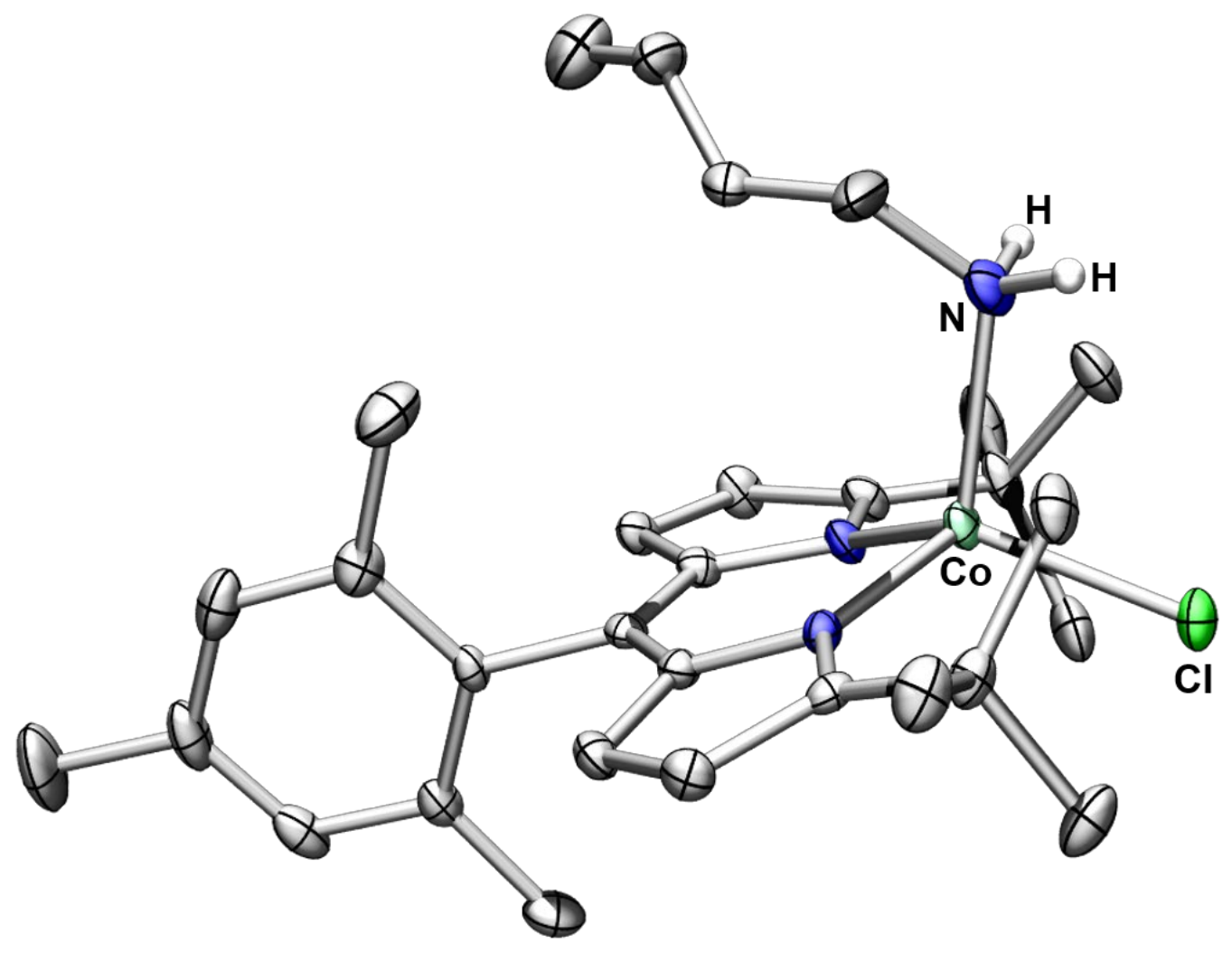

Figure S-46. Solid-state molecular structure for $\left({ }^{t \mathrm{Bu}} \mathrm{L}\right) \mathrm{CoCl}\left(n-\mathrm{BuNH}_{2}\right)(\mathbf{2 1})$ with thermal ellipsoids at $50 \%$ probability level. Hydrogens omitted for clarity, except for the amine $\mathrm{N}-H$. 
Computational Methods. Single point calculations and property calculations for complex $\mathbf{3}$ were carried out utilizing the ORCA $3 \cdot 0.3^{17}$ and package. All geometries were taken from the X-ray structure of compound 3 and the B3LYP ${ }^{18-19}$ functional was used with the def2-TZVP $(\mathrm{Co}, \mathrm{N}, \mathrm{Br})$ and def2-SV(P) $(\mathrm{C}, \mathrm{H})$ basis sets. ${ }^{20-22}$ Additionally, the def2-TZVP/J (Co, N, Br) and def2-SVP/J $(\mathrm{C}, \mathrm{H})$ auxiliary basis sets were employed to utilize the RIJCOSX approximation for accelerating the calculation. ${ }^{23}$ Geometry optimizations for complex $\mathbf{8}-\mathbf{N}_{2}$ considering all possible electronic configuration scenarios were carried out using ORCA 4.0.1.2 program. ${ }^{24}$ To minimize computational effort and facilitate convergence while retaining the overall structural environment of the molecule, the para-substituted phenyl groups of the 2,4,6-triphenylphenyl substituents were truncated to methyl units, and $\mathrm{Br}$ ancillary ligand was replaced with a $\mathrm{Cl}$. The B3LYP ${ }^{18-19}$ functional was used with the def2-TZVP $(\mathrm{Co}, \mathrm{N}, \mathrm{Cl})$ and def2-SV(P) $(\mathrm{C}, \mathrm{H})$ basis sets. ${ }^{20-22}$ Additionally, the def2-TZVP/J $(\mathrm{Co}, \mathrm{N}, \mathrm{Cl})$ and def2-SVP/J $(\mathrm{C}, \mathrm{H})$ auxiliary basis sets were employed to utilize the RIJCOSX approximation for accelerating the calculation. ${ }^{23}$

Summary of results. We have performed theoretical studies on the proposed Co-nitrene complex with $\mathbf{8}-\mathbf{N}_{2}$ as a model complex using ORCA (B3LYP functional, def2-TZVP for $\mathrm{Co}, \mathrm{N}, \mathrm{Cl}$ and def2-SVP/J for C, H). To minimize computational effort and facilitate convergence while retaining the overall structural environment of the molecule, the para-substituted phenyl groups of the 2,4,6triphenylphenyl substituents were truncated to methyl units, and $\mathrm{Br}$ ancillary ligand was replaced with a $\mathrm{Cl}$. As there is no direct spectroscopic evidence to assess either the formal oxidation state of the metal center $\left(\mathrm{Co}^{3+} v s . \mathrm{Co}^{4+}\right)$ or the electronic configuration of the compound (the spin-state of $\mathrm{Co}$ and the nature of the nitrene (NR) unit (e.g., imido $\left(\mathrm{NR}^{2-}\right)$, iminyl $\left({ }^{2} \mathrm{NR}^{1-}\right)$, nitrene adduct $\left({ }^{3} \mathrm{NR}\right)$ ), we have performed geometry optimizations for $\mathbf{8}-\mathbf{N}_{2}$ considering all possible electronic configuration scenarios. First, in the case of Co(IV) imido (which is equivalent with the bound nitrene unit being a dianionic imido $\left(\mathrm{NR}^{2-}\right)$ ), we have considered the $S=1 / 2,3 / 2$, and $5 / 2$ spin states $\left(\mathrm{Co}^{4+}=\mathrm{d}^{5}\right)$. Among these, the $S=1 / 2$ spin state is about 6.214 and $3.579 \mathrm{kcal} / \mathrm{mol}$ more stable with respect to the $S=3 / 2$ and $S=5 / 2$ states, respectively, suggesting that 8-N $\mathbf{N}_{2}$ might be best described as a doublet. However, we found that there is significant spin density localized on the $\mathrm{N}_{\alpha}$. Specifically, Mulliken spin population analysis suggests $+2.45(\alpha)$ spin density on the Co and $-1.55(\beta)$ spin density on the $\mathrm{N}_{\alpha}$, indicating that the nitrene (NR) moiety is more likely an open-shell iminyl $\left({ }^{2} \mathrm{NR}^{1-}\right)$ as opposed to a closed-shell imido $\left(\mathrm{NR}^{2-}\right)$, and consequently, the 
oxidation state of the Co would be $3+$ rather than $4+$. To this end, we have conducted a brokensymmetry calculation on $\mathbf{8}-\mathbf{N}_{2}$ at $S=1$ and $S=2$ spin states for a $\mathrm{d}^{6} \mathrm{Co}$ (III) center along with an $S=1 / 2$ nitrene moiety (We note that a low-spin $S=0$ state Co(III) along with an $S=1 / 2$ NR is equivalent to the previous $S=1 / 2 \mathrm{Co}(\mathrm{IV})$ case as a computational input). As a result, in both cases, the calculation converged to the broken symmetry solution, suggesting anti-ferromagnetic coupling state between the Co and NR fragment to result in a net doublet $\left(S_{\mathrm{Co}}(1)-S_{\mathrm{NR}}(1 / 2)\right)$ and quartet state $\left(S_{\mathrm{Co}}(2)-S_{\mathrm{NR}}(1 / 2)\right)$, respectively. Furthermore, the anti-ferromagnetically coupled doublet state was found to be more stable than the overall quartet spin state by $5.513 \mathrm{kcal} / \mathrm{mol}$. Therefore, we believe these computational results are in accord with our proposal suggesting that 8-N $\mathbf{N}_{\mathbf{2}}$ is more likely a Co(III) iminyl as opposed to a Co(IV) imido. However, to better understand the exact spin configuration of the compound (e.g., low-spin Co(III) vs. intermediate-spin Co(III) vs. high-spin $\mathrm{Co}(\mathrm{III})$ ), in-depth spectroscopic analysis using X-ray absorption or EPR spectroscopies are necessary and we are currently pursuing such spectroscopic characterization of this species. 
Table S-4. Summary of the geometry optimized results of $\mathbf{8}-\mathbf{N}_{2}$ as $\operatorname{Co}(\mathrm{IV})$ imido at the $S=1 / 2$, $3 / 2$, and $5 / 2$ spin states.

\begin{tabular}{c||ccc} 
& $\boldsymbol{S}=\mathbf{1} / \mathbf{2}$ model & $\boldsymbol{S}=\mathbf{3} / \mathbf{2}$ model & $\boldsymbol{S}=\mathbf{5} / \mathbf{2}$ model \\
\hline \hline Co- $\mathrm{N}_{\alpha}(\AA)$ & 1.877 & 1.764 & 1.95 \\
Spin density on Co & +2.453 & +2.659 & +2.742 \\
Spin density on $\mathrm{N}_{\alpha}$ & -1.545 & -0.035 & +1.744 \\
Relative energy $(\mathrm{kcal} / \mathrm{mol})$ & 0 & +6.214 & +3.579
\end{tabular}

Table S-5. Summary of the broken-symmetry ${ }^{25}$ geometry optimized results of $\mathbf{8}-\mathbf{N}_{\mathbf{2}}$ as $\mathrm{Co}$ (III) iminyl at the $S=1$ and $S=2$ spin states.

\begin{tabular}{c||cc} 
& $\begin{array}{c}\boldsymbol{S = 1} \text { Co and } \boldsymbol{S = 1 / 2} \text { NR } \\
\text { model }\end{array}$ & $\begin{array}{c}\boldsymbol{S = 2} \text { Co and } \boldsymbol{S}=\mathbf{1} / \mathbf{2} \text { NR } \\
\text { model }\end{array}$ \\
\hline \hline Co- $\mathrm{N}_{\alpha}(\AA)$ & 1.883 & 1.764 \\
Spin density on Co & +2.465 & +2.659 \\
Spin density on $\mathrm{N}_{\alpha}$ & -1.552 & -0.037 \\
Relative energy $(\mathrm{kcal} / \mathrm{mol})$ & 0 & +5.513
\end{tabular}



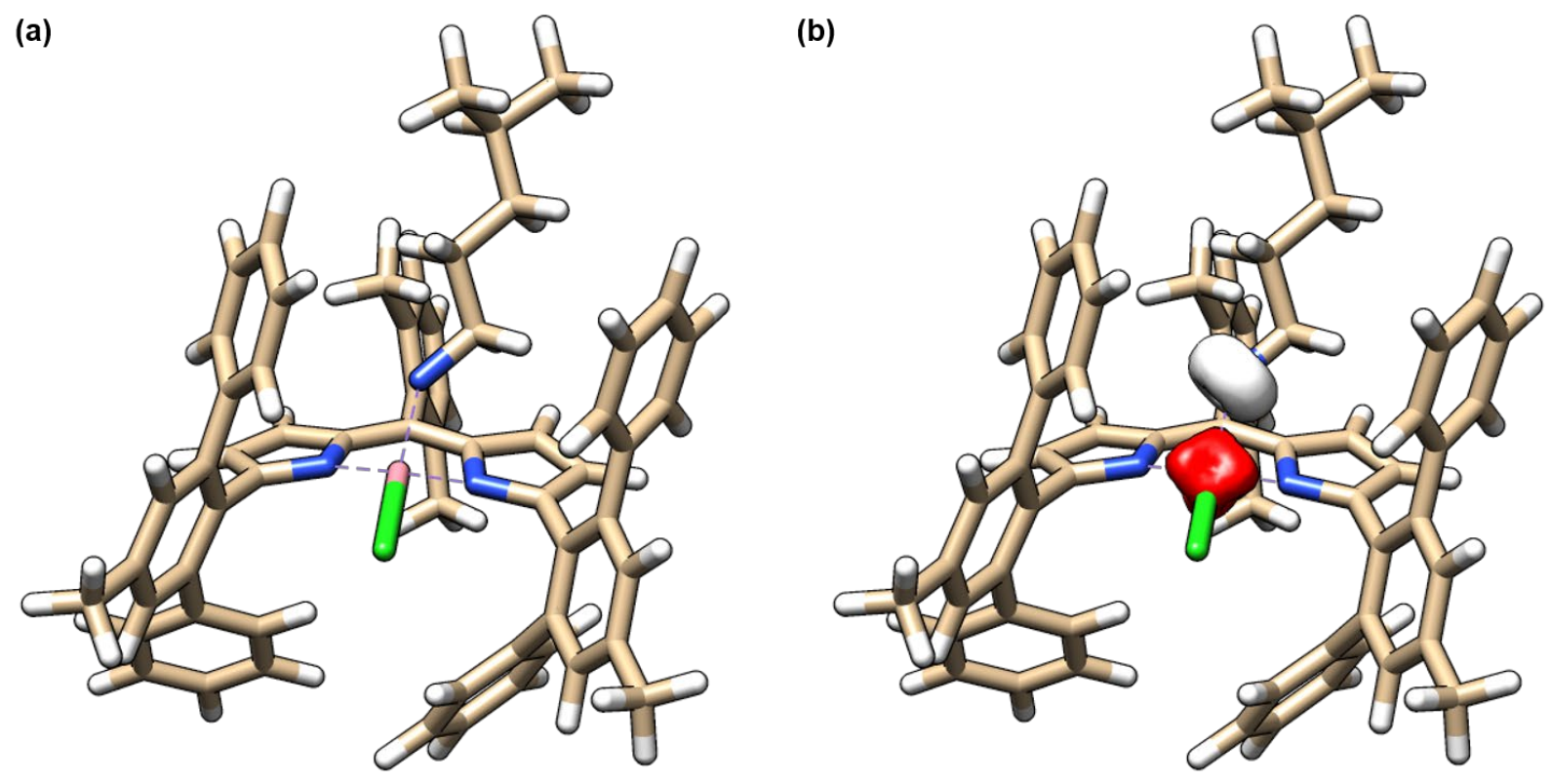

Figure S-47. Geometry optimized molecular structure and Mulliken spin density plot ( $\alpha-\beta)$ of $\mathbf{8}-\mathbf{N}_{2}$ at an $S=1 / 2$ spin state of $\mathrm{Co}(\mathrm{IV})$.
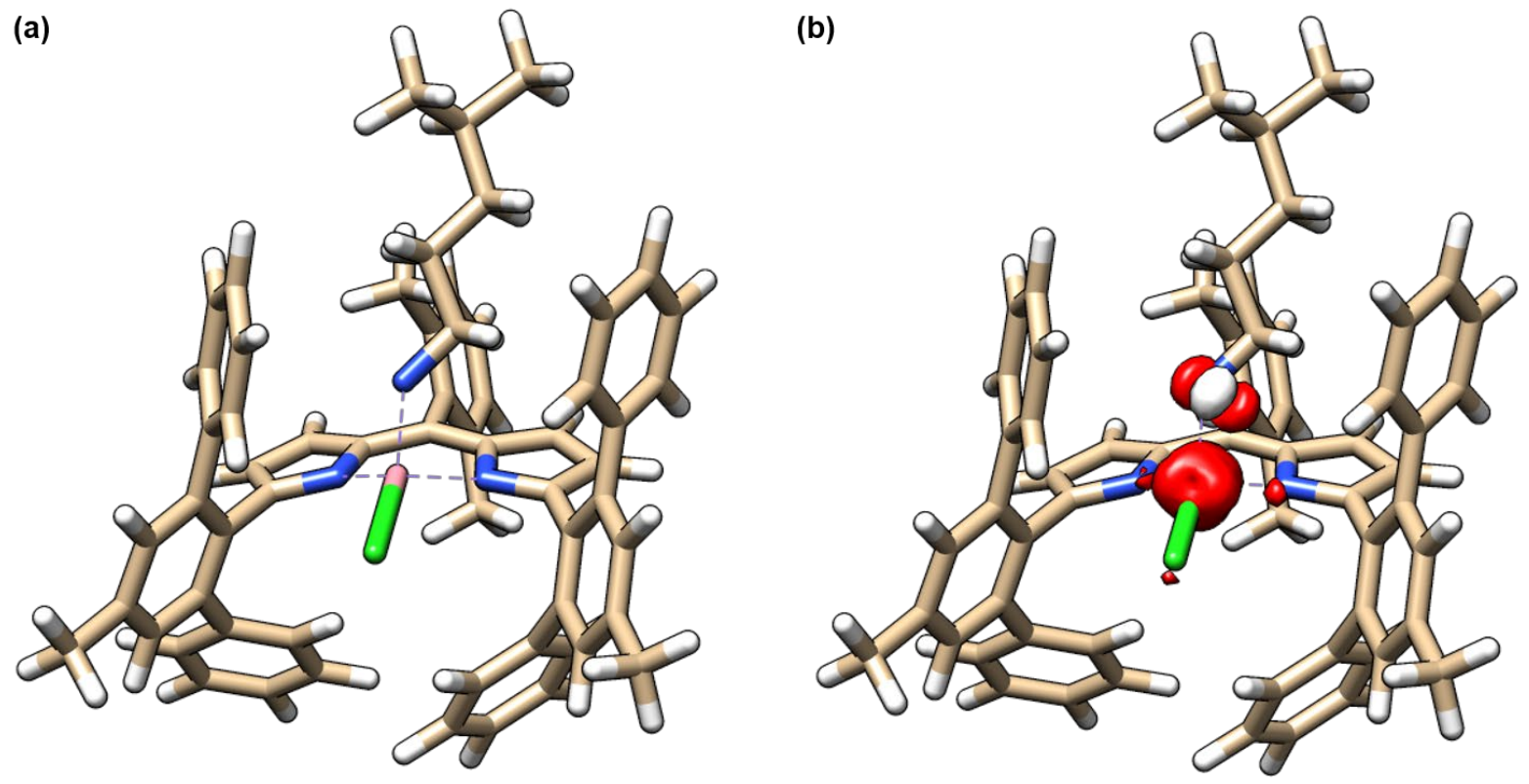

Figure S-48. Geometry optimized molecular structure and Mulliken spin density plot $(\alpha-\beta)$ of $\mathbf{8}-\mathbf{N}_{2}$ at an $S=3 / 2$ spin state of $\mathrm{Co}(\mathrm{IV})$. 
(a)

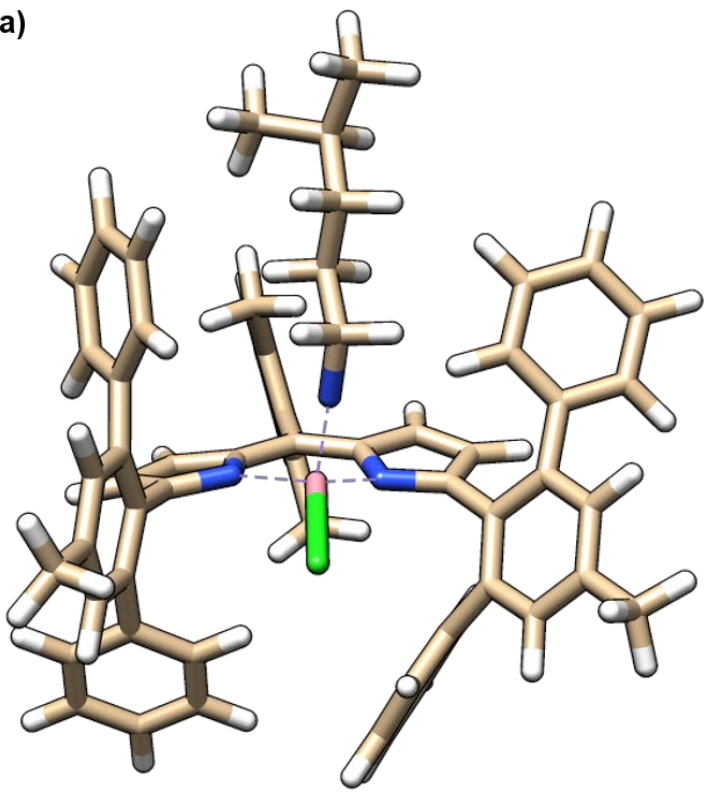

(b)

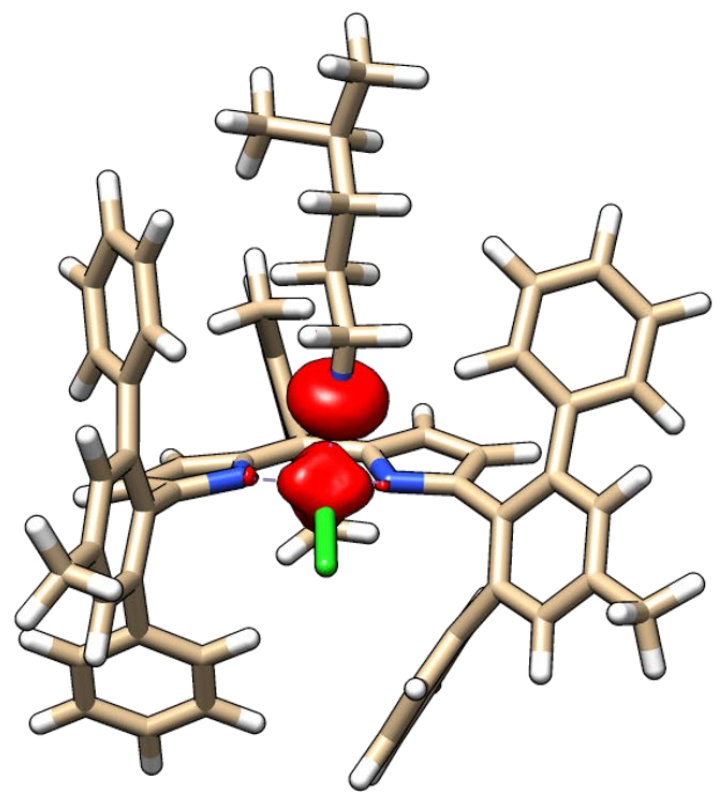

Figure S-49. Geometry optimized molecular structure and Mulliken spin density plot ( $\alpha-\beta)$ of $\mathbf{8}-\mathbf{N}_{2}$ at an $S=5 / 2$ spin state of $\mathrm{Co}(\mathrm{IV})$.

(a)

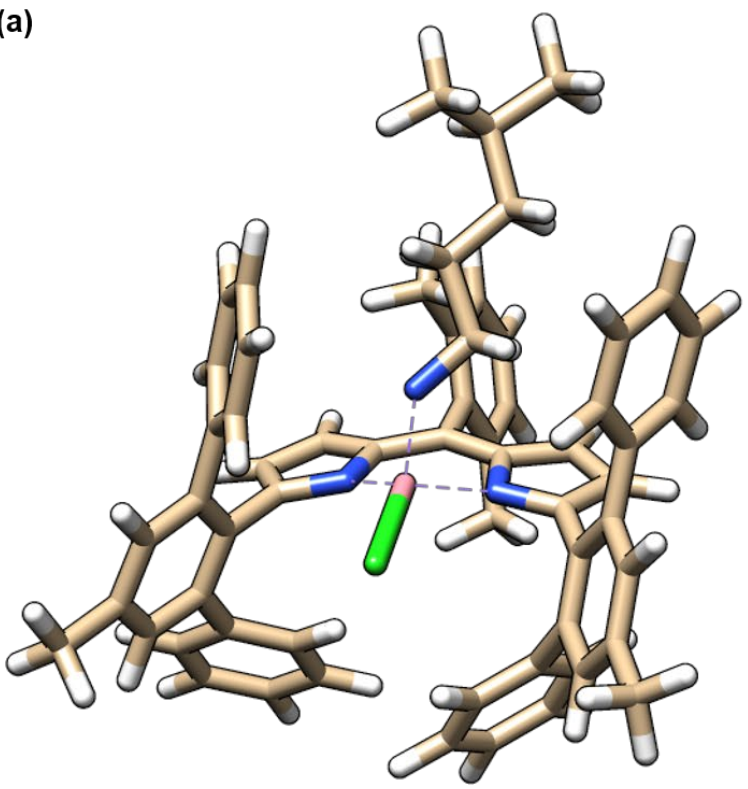

(b)

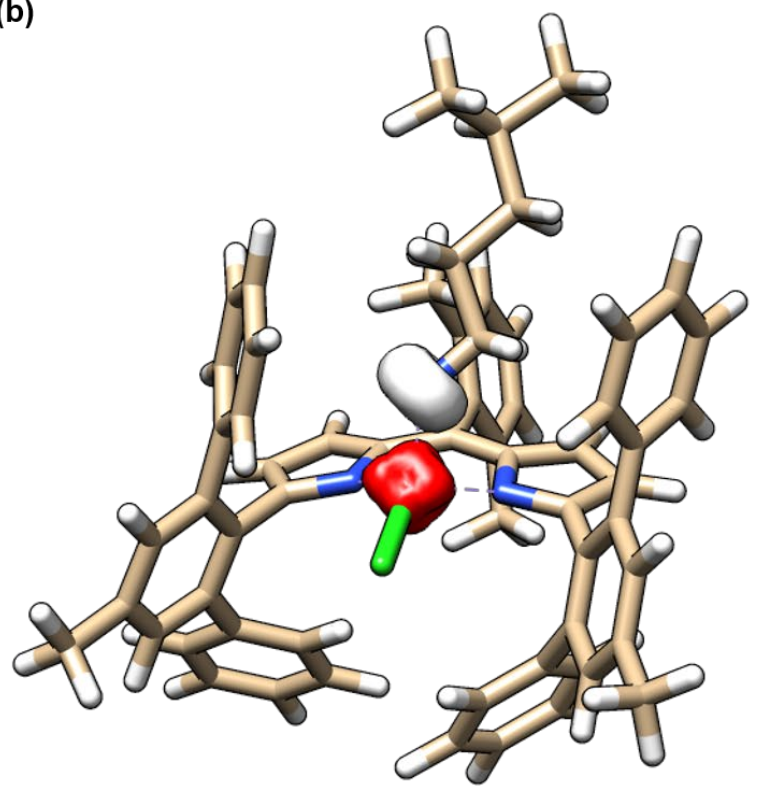

Figure S-50. Geometry optimized molecular structure and Mulliken spin density plot $(\alpha-\beta)$ of $\mathbf{8}-\mathbf{N}_{2}$ at an $S=1$ spin state of Co(III). 

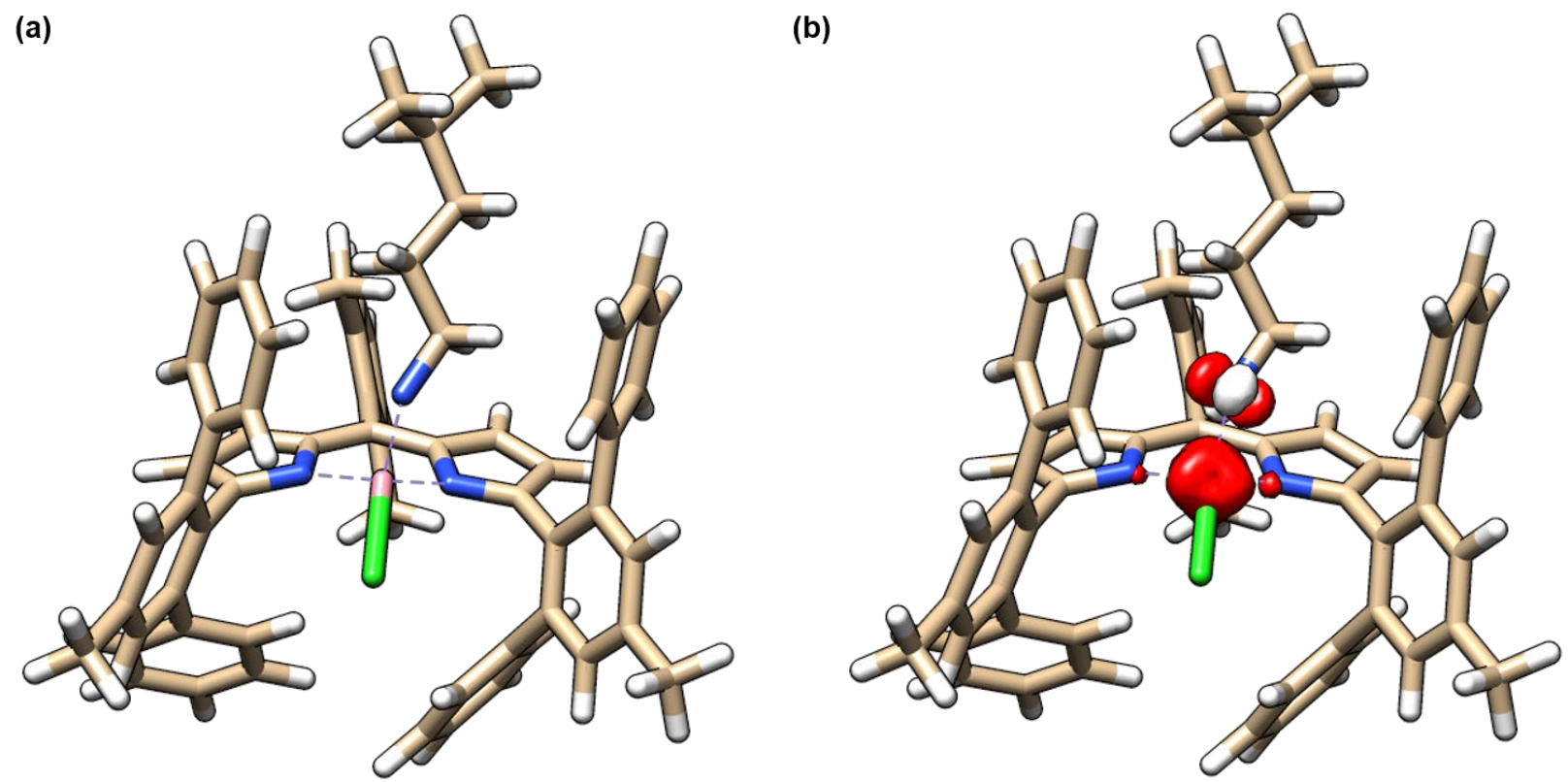

Figure S-51. Geometry optimized molecular structure and Mulliken spin density plot $(\alpha-\beta)$ of $\mathbf{8}-\mathbf{N}_{2}$ at an $S=2$ spin state of $\mathrm{Co}(\mathrm{III})$

Table S-6. Coordinates of optimized molecular structure for the truncated Co-nitrenoid $\left(\mathbf{8}-\mathbf{N}_{2}\right)$ at low-spin Co(IV) state.

Doublet $(S=1 / 2)$

$\begin{array}{lccc}\text { Co } & 6.181532 & 18.346355 & 7.941508 \\ \mathrm{~N} & 5.186259 & 19.891963 & 7.181055 \\ \mathrm{~N} & 5.703836 & 17.072267 & 6.467815 \\ \mathrm{C} & 6.248266 & 15.927470 & 6.035387 \\ \mathrm{~N} & 4.981662 & 18.001664 & 9.343581 \\ \mathrm{C} & 3.979178 & 19.612167 & 6.532219 \\ \mathrm{C} & 8.648817 & 15.362445 & 5.620667 \\ \mathrm{C} & 7.548478 & 15.389504 & 6.518886 \\ \mathrm{C} & 4.507094 & 17.264032 & 5.766980 \\ \mathrm{C} & 5.161939 & 21.184095 & 7.569959 \\ \mathrm{C} & 5.406742 & 21.932379 & 10.584083 \\ \mathrm{C} & 8.123159 & 17.151707 & 3.900301 \\ \mathrm{C} & 3.180580 & 20.793001 & 6.551705 \\ \mathrm{C} & 7.438383 & 22.196799 & 7.313491 \\ \mathrm{C} & 7.470363 & 21.913653 & 5.844642 \\ \mathrm{C} & 4.323632 & 16.153110 & 4.878732 \\ \mathrm{C} & 2.335512 & 18.312329 & 5.178468 \\ \mathrm{C} & 6.346910 & 21.876874 & 8.163983\end{array}$




\begin{tabular}{|c|c|c|c|}
\hline & 674581 & 18.393660 & \\
\hline & & 22.417274 & \\
\hline & 9.888129 & 14.872586 & 6 \\
\hline & 7.718298 & 14.887167 & \\
\hline & 8.986848 & 14.423613 & \\
\hline & 7.593818 & 22.870228 & \\
\hline & 6.438275 & 22.224426 & \\
\hline & 2.121354 & 18.96 & \\
\hline & 0.847098 & 18.89 & \\
\hline & 5.422096 & 15.3 & \\
\hline & 10.08 & & \\
\hline & 5199 & 15.8 & 4.20 \\
\hline & 6.60 & & \\
\hline & $8.5^{\prime}$ & 22.8 & \\
\hline & & 23. & \\
\hline 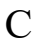 & 1.28 & 17.5 & \\
\hline & 8.0316 & $17.5^{\prime}$ & \\
\hline C & 0.0 & 17. & \\
\hline & 5.85 & 14.9 & 11. \\
\hline $\mathrm{C}$ & & 21. & \\
\hline & 74 & 15.12 & 10.1 \\
\hline$C$ & 40 & 21.2 & \\
\hline & 306 & 14. & \\
\hline C & 5.3 & 14.2 & \\
\hline & 6.54 & 22.4 & \\
\hline C & 4.61 & 14.3 & 10. \\
\hline C & & & \\
\hline & 8.53 & 21.1 & \\
\hline $\mathrm{C}$ & 91 & 22.3 & \\
\hline & 3.86 & 18.6 & \\
\hline $\mathrm{C}$ & 1.71 & 18.3 & 11.2 \\
\hline & 4.922 & 21.0 & 12.8 \\
\hline $\mathrm{C}$ & 2.96 & 17.6 & 10. \\
\hline $\mathrm{C}$ & 2865 & 16.8 & \\
\hline $\mathrm{C}$ & 8.34 & 16.7 & \\
\hline $\mathrm{C}$ & 3.203 & 22.24 & 11.59 \\
\hline $\mathrm{C}$ & & 21.6 & \\
\hline $\mathrm{C}$ & 3.618757 & 21.586340 & 12.75459 \\
\hline & 8.676397 & 21.0 & \\
\hline $\mathrm{C}$ & 3.236717 & 19.691338 & 3.22223 \\
\hline $\mathrm{C}$ & 8.773500 & 15.405091 & \\
\hline $\mathrm{C}$ & 0.663793 & 17.404342 & 11.90415 \\
\hline $\mathrm{C}$ & 1.239998 & 16.561770 & 13.05201 \\
\hline $\mathrm{C}$ & -0.548668 & 18.219880 & 12.3815 \\
\hline $\mathrm{H}$ & 0.676277 & 19.398309 & 2.39635 \\
\hline $\mathrm{H}$ & -0.785122 & 16.996661 & 5.6618 \\
\hline
\end{tabular}




\begin{tabular}{|c|c|c|c|}
\hline & 1.977484 & 17.468882 & \\
\hline & 0.545951 & 16.460963 & \\
\hline & 2.171308 & 15.965303 & 949453 \\
\hline & 4.042538 & 18.994834 & \\
\hline & 2.859597 & 20.180859 & \\
\hline & 3.704408 & 20.462644 & \\
\hline & 3.479942 & 16.018766 & \\
\hline & 5.647971 & 14.410150 & \\
\hline & 9.199795 & 13.961618 & \\
\hline & 9.031664 & 14.7 & \\
\hline & 8.268319 & 17.0 & \\
\hline & 7.707916 & 18.600656 & \\
\hline & 7.88 & 17.849517 & \\
\hline & 10.729146 & 14.8 & \\
\hline & 786 & $14 .($ & \\
\hline & 5.16 & 13.9 & \\
\hline & 3.41 & 13.5 & \\
\hline & 385 & 14.1 & \\
\hline & 8 & 15.2 & 12. \\
\hline & 6 & 15.5 & 10. \\
\hline & 3.75 & 22.9 & \\
\hline & 2.18 & 22.64 & \\
\hline & 2.93 & 21.4 & \\
\hline & & 20.5 & \\
\hline & 6.81 & 20.8 & \\
\hline & 5.7207 & 23.0 & 5.3 \\
\hline & 5.96 & 22.8 & \\
\hline & 7.88 & 21.5 & \\
\hline & 9.50 & 20.4 & \\
\hline & 9.241752 & 20.680032 & \\
\hline & 9.386644 & 23.096756 & \\
\hline & 7.633 & 23.148847 & 11.0 \\
\hline & 3.650741 & 22.821817 & \\
\hline & 2.19 & 20.900466 & \\
\hline & 4.21 & 19.440889 & 10.5 \\
\hline $\mathrm{H}$ & 3.268407 & 19.120589 & 9.1 \\
\hline & 3.566 & 17.20 & \\
\hline $\mathrm{H}$ & 2.688465 & 16.802879 & 10.046504 \\
\hline & & 19.098 & \\
\hline $\mathrm{H}$ & 1.222977 & 18.896361 & 10.439069 \\
\hline & 0.313465 & 16.704106 & 11.119290 \\
\hline $\mathrm{H}$ & 2.023515 & 15.866062 & 12.701660 \\
\hline & 1.686741 & 17.206811 & 13.833182 \\
\hline & 0.451072 & 15.954112 & 13.533207 \\
\hline & -1.001772 & 18.795860 & 11.55330 \\
\hline & -1.331892 & 17.565404 & 12.80611 \\
\hline
\end{tabular}




$\begin{array}{lccr}\mathrm{H} & -0.253742 & 18.940689 & 13.167879 \\ \mathrm{Cl} & 8.312717 & 18.574888 & 8.494342 \\ \mathrm{C} & 9.898695 & 23.881182 & 9.738153 \\ \mathrm{H} & 9.629381 & 24.629327 & 10.504603 \\ \mathrm{H} & 10.579433 & 23.151686 & 10.219326 \\ \mathrm{H} & 10.469793 & 24.392952 & 8.944052 \\ \mathrm{C} & 11.436251 & 13.906494 & 7.816053 \\ \mathrm{H} & 11.703399 & 12.958910 & 7.310895 \\ \mathrm{H} & 12.235044 & 14.633618 & 7.582294 \\ \mathrm{H} & 11.455096 & 13.721125 & 8.903712 \\ \mathrm{C} & -0.196935 & 18.206436 & 3.970807 \\ \mathrm{H} & -1.186441 & 18.175448 & 3.502709\end{array}$

Table S-7. Coordinates of optimized molecular structure for the truncated Co-nitrenoid $\left(\mathbf{8}-\mathbf{N}_{2}\right)$ at intermediate-spin Co(IV) state.

\begin{tabular}{|c|c|c|c|}
\hline \multicolumn{4}{|c|}{ Quartet $(S=3 / 2)$} \\
\hline Co & 6.220597 & 18.384996 & 8.068425 \\
\hline $\mathrm{N}$ & 5.214858 & 19.916191 & 7.189722 \\
\hline $\mathrm{N}$ & 5.651378 & 17.038960 & 6.667357 \\
\hline $\mathrm{C}$ & 6.159123 & 15.849809 & 6.305175 \\
\hline $\mathrm{N}$ & 5.077328 & 18.211280 & 9.400098 \\
\hline $\mathrm{C}$ & 4.022097 & 19.633699 & 6.515872 \\
\hline $\mathrm{C}$ & 8.471748 & 15.210993 & 5.650748 \\
\hline $\mathrm{C}$ & 7.496950 & 15.311575 & 6.680238 \\
\hline $\mathrm{C}$ & 4.445043 & 17.206753 & 5.977825 \\
\hline $\mathrm{C}$ & 5.226517 & 21.238065 & 7.462379 \\
\hline $\mathrm{C}$ & 5.274778 & 22.069339 & 10.448494 \\
\hline $\mathrm{C}$ & 7.950503 & 17.080172 & 4.026886 \\
\hline $\mathrm{C}$ & 3.277573 & 20.842834 & 6.391399 \\
\hline $\mathrm{C}$ & 7.500851 & 22.282981 & 7.302251 \\
\hline $\mathrm{C}$ & 7.617879 & 21.955676 & 5.846066 \\
\hline $\mathrm{C}$ & 4.203528 & 16.033282 & 5.193311 \\
\hline $\mathrm{C}$ & 2.383037 & 18.330332 & 5.173916 \\
\hline $\mathrm{C}$ & 6.373486 & 21.954436 & 8.099137 \\
\hline $\mathrm{C}$ & 3.674740 & 18.381496 & 5.950790 \\
\hline $\mathrm{C}$ & 3.943985 & 22.477676 & 10.245303 \\
\hline $\mathrm{C}$ & 9.717915 & 14.633382 & 5.926415 \\
\hline $\mathrm{C}$ & 7.813688 & 14.829608 & 7.972799 \\
\hline $\mathrm{C}$ & 9.091755 & 14.287093 & 8.208958 \\
\hline $\mathrm{C}$ & 7.481752 & 23.033172 & 9.985877 \\
\hline $\mathrm{C}$ & 6.371261 & 22.344295 & 9.466592 \\
\hline $\mathrm{C}$ & 2.315803 & 18.915660 & 3.885949 \\
\hline $\mathrm{C}$ & 1.091227 & 18.896673 & 3.199871 \\
\hline $\mathrm{C}$ & 5.279815 & 15.194697 & 5.386977 \\
\hline
\end{tabular}




\begin{tabular}{|c|c|c|c|}
\hline & 10.054737 & 14.170211 & \\
\hline & 8.239370 & 15.724664 & 4.265312 \\
\hline & 6.846391 & 14.805105 & 9.110595 \\
\hline & 8.586416 & 22.969366 & 7.870777 \\
\hline & 8.599013 & 23.357985 & 9.212494 \\
\hline & 1.243798 & 17.708456 & 5.740363 \\
\hline C & 7.792115 & 17.557251 & 2.721994 \\
\hline & 0.038792 & 17.715469 & 5.020986 \\
\hline & 6.408459 & 15.128404 & 11.492428 \\
\hline & 4.038566 & 21.846573 & \\
\hline $\mathrm{C}$ & 7.248619 & 15.254456 & 10.3 \\
\hline C & 5.599699 & 21.485282 & 11.6 \\
\hline C & 4.722137 & 13433 & 10.0 \\
\hline z & 5.561839 & 14.245063 & \\
\hline$C$ & 6.771722 & 22.535538 & 4.8 \\
\hline $\mathrm{C}$ & 5.144140 & 14.545770 & 11.3 \\
\hline ' & 8.37 & 14.865583 & 3.15 \\
\hline $\mathrm{C}$ & $8.6^{\circ}$ & 21.144 & \\
\hline $\mathrm{C}$ & 6.98 & 22.318625 & 3.5 \\
\hline $\mathrm{C}$ & 3.8 & 18.66 & \\
\hline $\mathrm{C}$ & 1.677806 & 18.223764 & 11.1 \\
\hline $\mathrm{C}$ & 4.63 & 21.3 & \\
\hline $\mathrm{C}$ & 2.95 & 17.5 & 10.5 \\
\hline $\mathrm{C}$ & 1.293 & 17.044918 & 7.0 \\
\hline $\mathrm{C}$ & 7.91 & 16.6 & \\
\hline & 2.97 & 1026 & 11.2 \\
\hline C & 8.0 & 16066 & 571 \\
\hline C & 3.32 & 21.753957 & 12.4 \\
\hline $\mathrm{C}$ & 8.89 & 20.922459 & 341 \\
\hline $\mathrm{C}$ & 3.53 & 19.5 & 3.2 \\
\hline $\mathrm{C}$ & 8.203683 & 15.340358 & \\
\hline $\mathrm{C}$ & 0.635791 & 17.250425 & 11.7 \\
\hline $\mathrm{C}$ & 1.198475 & 16.407941 & 12.9 \\
\hline $\mathrm{C}$ & -0.615694 & 18.023780 & 12.20 \\
\hline T & 1.033525 & 19.355589 & 2.20 \\
\hline $\mathrm{H}$ & -0.845780 & 17.243897 & 5.461611 \\
\hline $\mathrm{H}$ & 1.671615 & 17.736508 & 7.87 \\
\hline $\mathrm{H}$ & 0.290737 & 16.703772 & 7.407867 \\
\hline $\mathrm{H}$ & 1.963754 & 16.167555 & 7.102881 \\
\hline $\mathrm{H}$ & 4.366851 & 18.824053 & 3.160161 \\
\hline $\mathrm{H}$ & 3.289936 & 19.893232 & 2.216422 \\
\hline $\mathrm{H}$ & 3.909084 & 20.405369 & 3.807638 \\
\hline $\mathrm{H}$ & 3.343174 & 15.873395 & 4.545013 \\
\hline $\mathrm{H}$ & 5.467468 & 14.227373 & 4.922733 \\
\hline $\mathrm{H}$ & 8.603766 & 13.808382 & 3.328590 \\
\hline $\mathrm{H}$ & 8.304756 & 14.651841 & 1.009624 \\
\hline
\end{tabular}




\begin{tabular}{|c|c|c|c|}
\hline & 7.780499 & 17.067198 & \\
\hline & 7.577683 & 18.618910 & \\
\hline & .866691 & 17.769588 & \\
\hline & 10.453664 & & \\
\hline & 9.316619 & 13.903034 & \\
\hline & .227383 & 13.877028 & \\
\hline & .736895 & 13.651151 & 9.967648 \\
\hline & 4.492134 & 14.421733 & 242 \\
\hline & & 15.4 & \\
\hline & 8.2317 & 15.71 & 12 \\
\hline & & 22.9 & \\
\hline & 1.956413 & 22.66 & 11. \\
\hline & & 21.6 & \\
\hline & 4.924 & 20.8 & 13. \\
\hline & & 21.1 & \\
\hline & 5.9525 & 23.1 & 5.2 \\
\hline & 6 . & 22.7 & \\
\hline & & 21.3 & \\
\hline & 9.71 & 20.2 & 3.7 \\
\hline & & 20.6 & \\
\hline & 9.4 & 23.2 & \\
\hline & 7.45 & 23.3 & $11 .($ \\
\hline & & 22.9 & \\
\hline & 2.31 & 20.9 & \\
\hline & & 19.39 & $10 .^{\prime}$ \\
\hline & 3.342 & 19.259 & \\
\hline & 3.513 & 17.0 & 11.3 \\
\hline & $2.70 s$ & 16.8 & \\
\hline & 1.955 & 18.9 & 11. \\
\hline & & 18.80 & 10.7 \\
\hline & 0.330602 & 16.5 & 10. \\
\hline & 2.0153 & 15.7 & 12.5 \\
\hline & 1.596752 & 17.05 & 13.7 \\
\hline & 0.412 & 15.76 & 13. \\
\hline & -1.06 & 18.5 & \\
\hline & -1.39083 & 17.34 & 12.5 \\
\hline & -0.3666 & 18.7 & \\
\hline $\mathrm{Cl}$ & 8.412375 & 18.539655 & 882 \\
\hline & 9.7734 & 24.093023 & \\
\hline & 9.443941 & 24.828583 & 10.569961 \\
\hline & 10.463065 & 23.390082 & 10.3 \\
\hline & 10.356653 & 24.628829 & \\
\hline & 11.420207 & 13.575936 & 7.464437 \\
\hline & 11.682741 & 12.821065 & \\
\hline & 12.204664 & 14.355944 & 7.428152 \\
\hline & 11.473907 & 13.090055 & 8454156 \\
\hline
\end{tabular}


$\begin{array}{llll}\text { C } & -0.044029 & 18.313286 & 3.763511 \\ \mathrm{H} & -0.993785 & 18.320722 & 3.218564\end{array}$

Table S-8. Coordinates of optimized molecular structure for the truncated Co-nitrenoid $\left(\mathbf{8}-\mathbf{N}_{2}\right)$ at high-spin Co(IV) state.

$\begin{array}{lrrr}\text { Sextet }(S=5 / 2) & & \\ \text { Co } & 6.307190 & 18.595013 & 7.652618 \\ \text { N } & 5.162149 & 20.127499 & 6.956880 \\ \text { N } & 5.544725 & 17.155286 & 6.449617 \\ \text { C } & 5.994059 & 15.934279 & 6.103137 \\ \text { N } & 5.726175 & 18.278778 & 9.487293 \\ \text { C } & 3.940191 & 19.807870 & 6.345918 \\ \text { C } & 8.410153 & 15.287632 & 6.018844 \\ \text { C } & 7.180295 & 15.274251 & 6.722915 \\ \text { C } & 4.403489 & 17.409427 & 5.681178 \\ \text { C } & 5.113975 & 21.436198 & 7.277701 \\ \text { C } & 4.769041 & 22.843769 & 9.897497 \\ \text { C } & 8.527025 & 17.198296 & 4.340438 \\ \text { C } & 3.118881 & 20.978312 & 6.337256 \\ \text { C } & 7.464833 & 22.383988 & 7.190882 \\ \text { C } & 7.702676 & 21.981657 & 5.770158 \\ \text { C } & 4.172018 & 16.289024 & 4.824460 \\ \text { C } & 2.282633 & 18.506252 & 5.033246 \\ \text { C } & 6.236050 & 22.200913 & 7.883221 \\ \text { C } & 3.616500 & 18.578331 & 5.732076 \\ \text { C } & 4.212920 & 24.066401 & 10.321172 \\ \text { C } & 9.553895 & 14.721725 & 6.598066 \\ \text { C } & 7.105264 & 14.588302 & 7.963973 \\ \text { C } & 8.283826 & 14.048659 & 8.511574 \\ \text { C } & 7.116448 & 23.574968 & 9.701197 \\ \text { C } & 6.054545 & 22.851909 & 9.133802 \\ \text { C } & 2.113494 & 19.094787 & 3.757740 \\ \text { C } & 0.853023 & 19.022574 & 3.144246 \\ \text { C } & 5.183233 & 15.380350 & 5.070710 \\ \text { C } & 9.519634 & 14.123959 & 7.863555 \\ \text { C } & 8.507657 & 15.822591 & 4.622229 \\ \text { C } & 5.832052 & 14.290256 & 8.691902 \\ \text { C } & 8.497744 & 23.110916 & 7.807245 \\ \text { C } & 8.355987 & 23.698508 & 9.067387 \\ \text { C } & 1.204758 & 17.847338 & 5.674461 \\ \text { C } & 8.610375 & 17.649087 & 3.018271 \\ \text { C } & -0.040605 & 17.806272 & 5.028894 \\ \text { C } & 4.648955 & 13.934017 & 10.805052 \\ \text { C } & 3.851668 & 21.993646 & 6.917097\end{array}$




\begin{tabular}{|c|c|c|c|}
\hline & & & \\
\hline & & & \\
\hline & 3.567907 & 13.360291 & \\
\hline & 4.701236 & 13.780627 & \\
\hline & 6.799283 & & \\
\hline & 3.536944 & 13.427889 & 10. \\
\hline & & & \\
\hline & 8.922125 & 21.3 & \\
\hline & & & \\
\hline & 5.344 & 18.0 & 10. \\
\hline & & & \\
\hline & 2.95 & 21.6 & 11. \\
\hline & 3.8 & 17. & 10. \\
\hline & 1.3 & & \\
\hline & 8.6 & 16. & \\
\hline & 3.0 & 24.1 & 11. \\
\hline & 232 & 21. & \\
\hline & 2.4 & & 11. \\
\hline & 9.2 & & \\
\hline & & & \\
\hline & 8.6 & 15. & \\
\hline & 1.99 & $17 .($ & 12. \\
\hline & & & 12. \\
\hline & 1.7 & & 14.2 \\
\hline & & & \\
\hline & -0.8 & 827 & \\
\hline & & & \\
\hline & 0.3 & & \\
\hline & & & \\
\hline & & & \\
\hline & 2.952597 & & \\
\hline & & & \\
\hline & & & \\
\hline & & & \\
\hline & & & \\
\hline & 8.728561 & 14.6 & \\
\hline & & & \\
\hline & 8.621216 & 24988 & \\
\hline & .487409 & & \\
\hline & 10.495189 & & \\
\hline & 8.220561 & 13.507404 & \\
\hline & & & \\
\hline & 2.711750 & 12.956301 & 8.175661 \\
\hline & & & 10.675809 \\
\hline $\mathrm{H}$ & 4.642111 & & 11.89941 \\
\hline & 6.642152 & 14.743819 & 10.65029 \\
\hline
\end{tabular}




$\begin{array}{cccr}\mathrm{H} & 4.695534 & 25.002017 & 10.021443 \\ \mathrm{H} & 2.631042 & 25.064655 & 11.409544 \\ \mathrm{H} & 1.508481 & 22.936459 & 12.077021 \\ \mathrm{H} & 2.465325 & 20.754899 & 11.340424 \\ \mathrm{H} & 4.530682 & 20.699125 & 9.943312 \\ \mathrm{H} & 5.854460 & 22.821631 & 5.014060 \\ \mathrm{H} & 6.406219 & 22.436813 & 2.631197 \\ \mathrm{H} & 8.600275 & 21.412618 & 1.994221 \\ \mathrm{H} & 10.198133 & 20.720954 & 3.786216 \\ \mathrm{H} & 9.624278 & 21.073947 & 6.172914 \\ \mathrm{H} & 9.428934 & 23.261721 & 7.253141 \\ \mathrm{H} & 6.962475 & 24.050509 & 10.674330 \\ \mathrm{H} & 3.553032 & 23.031305 & 7.061747 \\ \mathrm{H} & 2.114732 & 21.041724 & 5.921068 \\ \mathrm{H} & 5.973317 & 17.196007 & 11.202894 \\ \mathrm{H} & 5.574938 & 18.922043 & 11.424710 \\ \mathrm{H} & 3.655837 & 16.746964 & 10.349063 \\ \mathrm{H} & 3.232988 & 18.464128 & 10.521398 \\ \mathrm{H} & 4.105912 & 16.608857 & 12.836693 \\ \mathrm{H} & 3.744306 & 18.332598 & 12.997808 \\ \mathrm{H} & 1.381901 & 17.898521 & 12.256237 \\ \mathrm{H} & 1.654593 & 15.737785 & 10.995066 \\ \mathrm{H} & 2.130714 & 14.919609 & 12.506339 \\ \mathrm{H} & 0.478407 & 15.568026 & 12.324435 \\ \mathrm{H} & 1.986750 & 18.070350 & 14.677440 \\ \mathrm{H} & 0.685691 & 16.864050 & 14.452552 \\ \mathrm{H} & 2.363607 & 16.327517 & 14.723195 \\ \mathrm{Cl} & 8.522782 & 18.692611 & 7.834301 \\ \mathrm{C} & 9.498409 & 24.445004 & 9.714335 \\ \mathrm{H} & 9.135665 & 25.201801 & 10.431739 \\ \mathrm{H} & 10.158790 & 23.752106 & 10.271935 \\ \mathrm{H} & 10.124422 & 24.957482 & 8.962260 \\ \mathrm{C} & 10.768857 & 13.567633 & 8.507594 \\ \mathrm{H} & 11.435732 & 13.093675 & 7.765424 \\ \mathrm{H} & 11.348382 & 14.371677 & 9.002437 \\ \mathrm{H} & 10.524304 & 12.817450 & 9.279216 \\ \mathrm{C} & -0.219898 & 18.390902 & 3.774379 \\ \mathrm{H} & -1.198382 & 18.352158 & 3.283925\end{array}$

Table S-9. Coordinates of optimized molecular structure for the truncated Co-nitrenoid $\left(\mathbf{8}-\mathbf{N}_{2}\right)$ at intermediate-spin Co(III) state. Broken-Symmetry calculation.

Quartet $(S=3 / 2)$

$(2,1)$

Co $\quad 6.180778 \quad 18.317814 \quad 8.003082$ 


$\begin{array}{cccr}\mathrm{N} & 5.176850 & 19.873019 & 7.259372 \\ \mathrm{~N} & 5.728468 & 17.040019 & 6.511690 \\ \mathrm{C} & 6.253184 & 15.887288 & 6.071689 \\ \mathrm{~N} & 4.971833 & 17.940741 & 9.396691 \\ \mathrm{C} & 4.000923 & 19.596583 & 6.553570 \\ \mathrm{C} & 8.568187 & 15.275365 & 5.382053 \\ \mathrm{C} & 7.600058 & 15.357002 & 6.419473 \\ \mathrm{C} & 4.497539 & 17.214071 & 5.862226 \\ \mathrm{C} & 5.165905 & 21.183332 & 7.583649 \\ \mathrm{C} & 5.108429 & 22.074873 & 10.559990 \\ \mathrm{C} & 7.946156 & 17.114090 & 3.753948 \\ \mathrm{C} & 3.237855 & 20.797866 & 6.467558 \\ \mathrm{C} & 7.470665 & 22.168919 & 7.508107 \\ \mathrm{C} & 7.646528 & 21.805695 & 6.066767 \\ \mathrm{C} & 4.265121 & 16.077300 & 5.020454 \\ \mathrm{C} & 2.367597 & 18.298921 & 5.197926 \\ \mathrm{C} & 6.297888 & 21.889026 & 8.256926 \\ \mathrm{C} & 3.688123 & 18.362423 & 5.926319 \\ \mathrm{C} & 3.800955 & 22.537245 & 10.325316 \\ \mathrm{C} & 9.825885 & 14.717674 & 5.648574 \\ \mathrm{C} & 7.929680 & 14.867840 & 7.706927 \\ \mathrm{C} & 9.217133 & 14.344714 & 7.932495 \\ \mathrm{C} & 7.347529 & 22.985351 & 10.167852 \\ \mathrm{C} & 6.244818 & 22.311176 & 9.613557 \\ \mathrm{C} & 8.687858 & 20.935917 & 5.695316 \\ \mathrm{C} & 2.224894 & 18.907711 & 3.927760 \\ \mathrm{C} & 0.971100 & 18.868027 & 3.297581 \\ \mathrm{C} & 5.368550 & 15.260850 & 5.136962 \\ \mathrm{C} & 10.177261 & 14.253266 & 6.921150 \\ \mathrm{C} & 8.312234 & 15.777981 & 3.997299 \\ \mathrm{C} & 6.960401 & 14.813260 & 8.842191 \\ \mathrm{C} & 8.545670 & 22.842853 & 8.109187 \\ \mathrm{C} & 8.506903 & 23.264420 & 9.440373 \\ \mathrm{C} & 1.271406 & 17.637304 & 5.803595 \\ \mathrm{C} & 7.754479 & 17.575450 & 2.448150 \\ \mathrm{C} & 0.034695 & 17.625090 & 5.140872 \\ \mathrm{C} & 6.501134 & 15.108367 & 11.222809 \\ \mathrm{C} & 3.975619 & 21.790366 & 7.092773 \\ \mathrm{C} & 7.352455 & 15.245282 & 10.123756 \\ \mathrm{C} & 5.377618 & 21.474292 & 11.808443 \\ \mathrm{C} & 5.678136 & 14.252126 & 8.694059 \\ \mathrm{C} & & 22.163069 & 3.705432\end{array}$




$\begin{array}{cccr}\mathrm{C} & 3.900130 & 18.594161 & 10.031329 \\ \mathrm{C} & 1.696546 & 18.350994 & 11.289311 \\ \mathrm{C} & 4.385802 & 21.353486 & 12.785731 \\ \mathrm{C} & 2.919026 & 17.614539 & 10.722872 \\ \mathrm{C} & 1.408038 & 16.941209 & 7.139555 \\ \mathrm{C} & 7.918992 & 16.712077 & 1.359935 \\ \mathrm{C} & 2.804463 & 22.413013 & 11.299042 \\ \mathrm{C} & 8.208044 & 21.303219 & 3.348218 \\ \mathrm{C} & 3.092354 & 21.827825 & 12.536656 \\ \mathrm{C} & 8.964090 & 20.682691 & 4.348427 \\ \mathrm{C} & 3.393233 & 19.572473 & 3.231682 \\ \mathrm{C} & 8.288534 & 15.382909 & 1.587780 \\ \mathrm{C} & 0.603803 & 17.453071 & 11.906583 \\ \mathrm{C} & 1.128938 & 16.582792 & 13.058142 \\ \mathrm{C} & -0.583179 & 18.312040 & 12.371834 \\ \mathrm{H} & 0.855140 & 19.343917 & 2.318756 \\ \mathrm{H} & -0.816553 & 17.121901 & 5.611379 \\ \mathrm{H} & 1.840153 & 17.612258 & 7.901965 \\ \mathrm{H} & 0.427599 & 16.591033 & 7.505570 \\ \mathrm{H} & 2.077337 & 16.065170 & 7.074093 \\ \mathrm{H} & 4.191715 & 18.842183 & 3.007396 \\ \mathrm{H} & 3.073134 & 20.030331 & 2.280721 \\ \mathrm{H} & 3.852545 & 20.360085 & 3.851656 \\ \mathrm{H} & 3.388872 & 15.925983 & 4.391587 \\ \mathrm{H} & 5.566742 & 14.324428 & 4.617068 \\ \mathrm{H} & 8.778650 & 13.881819 & 3.065127 \\ \mathrm{H} & 8.425862 & 14.697848 & 0.744905 \\ \mathrm{H} & 7.760834 & 17.077384 & 0.339499 \\ \mathrm{H} & 7.475635 & 18.621164 & 2.285465 \\ \mathrm{H} & 7.822202 & 17.802915 & 4.594169 \\ \mathrm{H} & 10.558569 & 14.665857 & 4.836025 \\ \mathrm{H} & 9.453822 & 13.956325 & 8.927338 \\ \mathrm{H} & 5.353015 & 13.891840 & 7.714676 \\ \mathrm{H} & 3.842128 & 13.650985 & 9.656061 \\ \mathrm{H} & 4.572612 & 14.406529 & 11.926310 \\ \mathrm{H} & 6.832668 & 15.458411 & 12.206265 \\ \mathrm{H} & 8.330770 & 15.714097 & 10.252235 \\ \mathrm{H} & 3.565908 & 23.035640 & 9.385236 \\ \mathrm{H} & 1.800951 & 22.799050 & 11.092032 \\ \mathrm{H} & 4.628965 & 20.883584 & 13.744733 \\ \mathrm{H} & 6.076526 & 23.098795 & 5.324829 \\ \mathrm{H} & 21.6939 & 21.119172 & 2.293354 \\ \mathrm{H} & 19.997669 & 4.078772\end{array}$




$\begin{array}{cccr}\mathrm{H} & 9.274954 & 20.446790 & 6.477094 \\ \mathrm{H} & 9.427811 & 23.062189 & 7.499280 \\ \mathrm{H} & 7.278706 & 23.323520 & 11.206068 \\ \mathrm{H} & 3.726830 & 22.849221 & 7.159781 \\ \mathrm{H} & 2.280227 & 20.907102 & 5.960996 \\ \mathrm{H} & 4.313981 & 19.308583 & 10.777480 \\ \mathrm{H} & 3.359948 & 19.214822 & 9.281587 \\ \mathrm{H} & 3.464827 & 17.077356 & 11.516514 \\ \mathrm{H} & 2.602658 & 16.851441 & 9.990189 \\ \mathrm{H} & 2.033498 & 19.080878 & 12.050902 \\ \mathrm{H} & 1.237556 & 18.952150 & 10.479357 \\ \mathrm{H} & 0.237802 & 16.772903 & 11.111174 \\ \mathrm{H} & 1.896459 & 15.864506 & 12.718706 \\ \mathrm{H} & 1.579007 & 17.208710 & 13.853037 \\ \mathrm{H} & 0.310646 & 15.998237 & 13.517888 \\ \mathrm{H} & -1.007683 & 18.902780 & 11.539005 \\ \mathrm{H} & -1.393667 & 17.686823 & 12.789409 \\ \mathrm{H} & -0.271183 & 19.022844 & 13.160910 \\ \mathrm{Cl} & 8.304894 & 18.541477 & 8.601375 \\ \mathrm{C} & 9.673621 & 23.980911 & 10.078493 \\ \mathrm{H} & 9.335075 & 24.712637 & 10.833367 \\ \mathrm{H} & 10.343370 & 23.264643 & 10.594035 \\ \mathrm{H} & 10.281217 & 24.517461 & 9.328761 \\ \mathrm{C} & 11.555253 & 13.684571 & 7.173212 \\ \mathrm{H} & 11.838352 & 12.953733 & 6.394264 \\ \mathrm{H} & 12.321233 & 14.483548 & 7.158807 \\ \mathrm{H} & 11.616925 & 13.178433 & 8.152215 \\ \mathrm{C} & -0.121052 & 18.242745 & 3.899808 \\ \mathrm{H} & -1.094026 & 18.233088 & 3.397480\end{array}$

Table S-10. Coordinates of optimized molecular structure for the truncated Co-nitrenoid $\left(\mathbf{8}-\mathbf{N}_{2}\right)$ at intermediate-spin Co(III) state. Broken-Symmetry calculation.

Sextet $(S=5 / 2)$

$(4,1)$

$\begin{array}{llll}\mathrm{Co} & 6.220640 & 18.389496 & 8.064970 \\ \mathrm{~N} & 5.204872 & 19.908176 & 7.176731 \\ \mathrm{~N} & 5.659763 & 17.032271 & 6.673826 \\ \mathrm{C} & 6.178826 & 15.846276 & 6.317957 \\ \mathrm{~N} & 5.081510 & 18.218291 & 9.400339 \\ \mathrm{C} & 4.010775 & 19.612547 & 6.510943 \\ \mathrm{C} & 8.502946 & 15.222392 & 5.690076 \\ \mathrm{C} & 7.516362 & 15.318997 & 6.708480 \\ \mathrm{C} & 4.455502 & 17.188725 & 5.978503 \\ \mathrm{C} & 5.207013 & 21.231162 & 7.443885\end{array}$




$\begin{array}{cccr}\text { C } & 5.276098 & 22.079513 & 10.424436 \\ \mathrm{C} & 7.979534 & 17.074966 & 4.047757 \\ \mathrm{C} & 3.254638 & 20.814488 & 6.387117 \\ \mathrm{C} & 7.473879 & 22.289045 & 7.258186 \\ \mathrm{C} & 7.581402 & 21.955787 & 5.802795 \\ \mathrm{C} & 4.228091 & 16.011921 & 5.194639 \\ \mathrm{C} & 2.380117 & 18.288409 & 5.177299 \\ \mathrm{C} & 6.354960 & 21.958570 & 8.066470 \\ \mathrm{C} & 3.673687 & 18.355816 & 5.949703 \\ \mathrm{C} & 3.942763 & 22.484696 & 10.231795 \\ \mathrm{C} & 9.751798 & 14.658990 & 5.982426 \\ \mathrm{C} & 7.823073 & 14.845440 & 8.006857 \\ \mathrm{C} & 9.103877 & 14.317299 & 8.259804 \\ \mathrm{C} & 7.475079 & 23.049912 & 9.939209 \\ \mathrm{C} & 6.363075 & 22.354351 & 9.432096 \\ \mathrm{C} & 2.301751 & 18.867755 & 3.887314 \\ \mathrm{C} & 1.075117 & 18.831752 & 3.205490 \\ \mathrm{C} & 5.310292 & 15.182582 & 5.395663 \\ \mathrm{C} & 10.079411 & 14.206581 & 7.265632 \\ \mathrm{C} & 8.277941 & 15.723856 & 4.299027 \\ \mathrm{C} & 6.841932 & 14.815389 & 9.132745 \\ \mathrm{C} & 8.560737 & 22.983363 & 7.814604 \\ \mathrm{C} & 8.583815 & 23.376835 & 9.154722 \\ \mathrm{C} & 1.249919 & 17.655969 & 5.750349 \\ \mathrm{C} & 7.824545 & 17.539786 & 2.738092 \\ \mathrm{C} & 0.042443 & 17.646144 & 5.035203 \\ \mathrm{C} & 7.999817 & 21.515364 & 3.045622 \\ \mathrm{C} & 6.371309 & 15.134978 & 11.508901 \\ \mathrm{C} & 4.010277 & 21.827200 & 6.955628 \\ \mathrm{C} & 7.225633 & 15.264944 & 10.411057 \\ \mathrm{C} & 5.613466 & 21.500496 & 11.666281 \\ \mathrm{C} & 4.706985 & 14.115630 & 10.083294 \\ \mathrm{C} & 5.561191 & 14.250501 & 8.985166 \\ \mathrm{C} & 6.712573 & 22.510939 & 4.846926 \\ \mathrm{C} & 5.110680 & 14.548737 & 11.351513 \\ \mathrm{C} & 8.425233 & 14.856464 & 3.201607 \\ \mathrm{C} & 8.655693 & 21.166372 & 5.352483 \\ \mathrm{C} & 6.920472 & 22.293111 & 3.480600 \\ \mathrm{C} & 3.894827 & 18.672693 & 9.991704 \\ \mathrm{C} & 1.683389 & 18.246658 & 11.164830 \\ \mathrm{C} & 2.962002 & 17.604250 & 10.604050 \\ \mathrm{C} & 1.312479 & 17.000065 & 7.111740 \\ \mathrm{C} & 21.768613 & 12.472879\end{array}$




\begin{tabular}{|c|c|c|c|}
\hline & 8.862500 & 20.945227 & \\
\hline & 3.508116 & & 228792 \\
\hline & 8.258501 & 15.318920 & 392090 \\
\hline & 0.639032 & 17.278330 & 1.759566 \\
\hline & .198894 & 16.441119 & \\
\hline & -0.611792 & & 12.203450 \\
\hline & 1.008621 & 19.285857 & 2.211865 \\
\hline & -0.835316 & 17.166367 & 5.480623 \\
\hline & 1.673485 & 17.704477 & 7.881465 \\
\hline & 0.31 & 16. & 7.42 \\
\hline & 2.00 & & \\
\hline & 4.3 & 351 & 3.15 \\
\hline & & & \\
\hline & 3.8 & 161 & 3.80 \\
\hline & 3.3 & & 4.54 \\
\hline & 5.508420 & 006 & 4.93 \\
\hline & 8.6 & & \\
\hline & & & \\
\hline & 7.82 & & 0.6 \\
\hline & & & \\
\hline & 13 & 767 & 4.8 \\
\hline & 10.45 & & \\
\hline & & & 9.2 \\
\hline & 5.24 & 13 & \\
\hline & 86 & 135 & 9.9 \\
\hline & 76 & 14. & 12.2 \\
\hline & 29 & 15. & 12.4 \\
\hline & 8.20 & 366 & 10.5 \\
\hline & 532 & 862 & 736 \\
\hline & 1.962736 & 22 . & 11.06 \\
\hline & 2.598543 & 21. & 13.268732 \\
\hline & 4.957379 & 20 . & 13.630922 \\
\hline & 6.635 & 21. & 11.8 \\
\hline & 5.882419 & 2596 & 5.174991 \\
\hline & 6.242366 & 22 . & 2.752018 \\
\hline & 8.172870 & 6258 & 1.975685 \\
\hline & 9.701011 & & 3.659541 \\
\hline & 9.325856 & 20.713000 & 6.087314 \\
\hline & 9.400153 & 13966 & \\
\hline & 7.452583 & 23.370960 & 10.984841 \\
\hline & 3.766651 & 22.889222 & 6.991112 \\
\hline & 2.285720 & 20.907714 & 5.898898 \\
\hline & 4.175193 & 19.414947 & 10.771791 \\
\hline & 3.347809 & 19.269022 & 9.223922 \\
\hline $\mathrm{H}$ & 3.517507 & 17.069393 & 11.392628 \\
\hline & 2.713008 & 16.853955 & 9.833655 \\
\hline
\end{tabular}




$\begin{array}{cccr}\mathrm{H} & 1.961946 & 18.986999 & 11.940481 \\ \mathrm{H} & 1.196608 & 18.828877 & 10.356716 \\ \mathrm{H} & 0.333782 & 16.577887 & 10.956917 \\ \mathrm{H} & 2.016288 & 15.773605 & 12.593227 \\ \mathrm{H} & 1.595752 & 17.092330 & 13.722677 \\ \mathrm{H} & 0.412022 & 15.804897 & 13.366292 \\ \mathrm{H} & -1.056515 & 18.622607 & 11.364203 \\ \mathrm{H} & -1.388405 & 17.377855 & 12.602266 \\ \mathrm{H} & -0.362507 & 18.782013 & 13.000785 \\ \mathrm{Cl} & 8.410579 & 18.562751 & 8.099914 \\ \mathrm{C} & 9.760860 & 24.118595 & 9.743527 \\ \mathrm{H} & 9.435719 & 24.853397 & 10.501391 \\ \mathrm{H} & 10.459263 & 23.419618 & 10.244465 \\ \mathrm{H} & 10.333185 & 24.656281 & 8.967489 \\ \mathrm{C} & 11.448805 & 13.630472 & 7.545986 \\ \mathrm{H} & 11.739526 & 12.891520 & 6.777661 \\ \mathrm{H} & 12.220657 & 14.423825 & 7.538196 \\ \mathrm{H} & 11.490324 & 13.131358 & 8.529711 \\ \mathrm{C} & -0.051427 & 18.237631 & 3.775434 \\ \mathrm{H} & -1.002919 & 18.231235 & 3.233493\end{array}$




\section{References.}

(1) King, E. R.; Hennessy, E. T.; Betley, T. A. Catalytic C-H bond amination from high-spin iron imido complexes. J. Am. Chem. Soc. 2011, 133 (13), 4917-4923.

(2) King, E. R.; Sazama, G. T.; Betley, T. A. Co(III) imidos exhibiting spin crossover and C-H bond activation. J. Am. Chem. Soc. 2012, 134 (43), 17858-17861.

(3) Baek, Y.; Betley, T. A. Catalytic C-H amination mediated by dipyrrin cobalt imidos. J. Am. Chem. Soc. 2019, 141 (19), 7797-7806.

(4) Smith, P. A. S.; Hall, J. H. Kinetic evidence for the formation of azene (electron-deficient nitrogen) intermediates from aryl azides. J. Am. Chem. Soc. 1962, 84 (3), 480-485.

(5) Jin, L.-M.; Lu, H.; Cui, Y.; Lizardi, C. L.; Arzua, T. N.; Wojtas, L.; Cui, X.; Zhang, X. P. Selective radical amination of aldehydic $\mathrm{C}\left(\mathrm{sp}^{2}\right)-\mathrm{H}$ bonds with fluoroaryl azides via $\mathrm{Co}(\mathrm{II})$-based metalloradical catalysis: synthesis of $N$-fluoroaryl amides from aldehydes under neutral and nonoxidative conditions. Chem. Sci. 2014, 5 (6), 2422-2427.

(6) Hennessy, E. T.; Betley, T. A. Complex $N$-heterocycle synthesis via iron-catalyzed, direct CH bond amination. Science 2013, 340 (6132), 591-595.

(7) Stoll, S.; Schweiger, A. EasySpin, a comprehensive software package for spectral simulation and analysis in EPR. J. Magn. Reson. 2006, 178 (1), 42-55.

(8) Johnson, E. R.; Keinan, S.; Mori-Sánchez, P.; Contreras-García, J.; Cohen, A. J.; Yang, W. Revealing noncovalent interactions. J. Am. Chem. Soc. 2010, 132 (18), 6498-6506.

(9) Contreras-García, J.; Johnson, E. R.; Keinan, S.; Chaudret, R.; Piquemal, J.-P.; Beratan, D. N.; Yang, W. NCIPLOT: A program for plotting noncovalent interaction regions. J. Chem. Theory Comput. 2011, 7 (3), 625-632.

(10) Intrieri, D.; Caselli, A.; Ragaini, F.; Macchi, P.; Casati, N.; Gallo, E. Insights into the mechanism of the ruthenium-porphyrin-catalysed allylic amination of olefins by aryl azides. Eur. J. Inorg. Chem. 2012, 569-580. 
(11) Fantauzzi, S.; Gallo, E.; Caselli, A.; Piangiolino, C.; Ragaini, F.; Cenini, S. The (porphyrin)ruthenium-catalyzed aziridination of olefins using aryl azides as nitrogen sources. Eur. J. Org. Chem. 2007, 6053-6059.

(12) Yoshimoto, F. K.; Jung, I. J.; Goyal, S.; Gonzalez, E.; Guengerich, F. P. Isotope-labeling studies support the electrophilic compound I iron active species, $\mathrm{FeO}^{3+}$, for the carbon-carbon bond cleavage reaction of the cholesterol side-chain cleavage enzyme, Cytochrome P450 11A1. J. Am. Chem. Soc. 2016, 138 (37), 12124-12141.

(13) Renny, J. S.; Tomasevich, L. L.; Tallmadge, E. H.; Collum, D. B. Method of continuous variations: applications of Job plots to the study of molecular associations in organometallic chemistry. Angew. Chem. Int. Ed. 2013, 52 (46), 11998-12013.

(14) Fielding, L. Determination of association constants $\left(K_{\mathrm{a}}\right)$ from solution NMR data. Tetrahedron 2000, 56 (34), 6151-6170.

(15) APEX2 Software Suite; Bruker AXS: Madison, WI, 2014.

(16) (a) Sheldrick, G. M. SHELXT - Integrated space-group and crystal-structure determination. Acta Crystallogr., Sect. A 2015, 71 (1), 3-8. (b) Sheldrick, G. M. Crystal structure refinement with SHELXL. Acta Crystallogr., 2015, C71, 3-8.

(17) Neese, F. ORCA - An ab initio, Density functional and semi-empirical electronic structure package; Version 3.0.3 ed. Universitat Bonn, Bonn, Germany, 2009.

(18) Lee, C.; Yang, W.; Parr, R. G. Development of the Colle-Salvetti correlation-energy formula into a functional of the electron density. Phys. Rev. B 1988, 37 (2), 785-789.

(19) Becke, A. D. Density-functional thermochemistry. III. The role of exact exchange. J. Chem. Phys. 1993, 98 (7), 5648-5652.

(20) Weigend, F.; Ahlrichs, R. Balanced basis sets of split valence, triple zeta valence and quadruple zeta valence quality for $\mathrm{H}$ to $\mathrm{Rn}$ : Design and assessment of accuracy. Phys. Chem. Chem. Phys. 2005, 7 (18), 3297-3305.

(21) Schäfer, A.; Huber, C.; Ahlrichs, R. Fully optimized contracted Gaussian basis sets of triple zeta valence quality for atoms Li to Kr. J. Chem. Phys. 1994, 100 (8), 5829-5835. 
(22) Schäfer, A.; Horn, H.; Ahlrichs, R. Fully optimized contracted Gaussian basis sets for atoms Li to Kr. J. Chem. Phys. 1992, 97 (4), 2571-2577.

(23) Weigend, F. Accurate coulomb-fitting basis sets for H to Rn. Phys. Chem. Chem. Phys. 2006, $8(9), 1057-1065$.

(24) Neese, F. ORCA - The ORCA program system, Wiley Interdiscip. Rev.: Comput. Mol. Sci., 2012, 2, 73 .

(25) Kirchner, B.; Wennmohs, F.; Ye, S.; Neese, F. Theoretical bioinorganic chemistry: the electronic structure makes a difference. Curr. Opin. Chem. Biol. 2007, 11 (2), 134-141. 


\section{checkCIF/PLATON report}

Structure factors have been supplied for datablock(s) 3_ArLCoBrN3Ar

THIS REPORT IS FOR GUIDANCE ONLY. IF USED AS PART OF A REVIEW PROCEDURE FOR PUBLICATION, IT SHOULD NOT REPLACE THE EXPERTISE OF AN EXPERIENCED CRYSTALLOGRAPHIC REFEREE.

No syntax errors found. CIF dictionary Interpreting this report

\section{Datablock: 3_ArLCoBrN3Ar}

\begin{tabular}{|c|c|c|c|}
\hline Bond precision: & $\mathrm{C}-\mathrm{C}=0.0081 \mathrm{I}$ & Wavel & ength $=0.71073$ \\
\hline Cell: & $\begin{array}{l}a=11.5371(4) \\
a l p h a=90\end{array}$ & $\begin{array}{l}\mathrm{b}=22.8606(10) \\
\text { bet } \mathrm{a}=94.279(4)\end{array}$ & $\begin{array}{l}\mathrm{C}=27.3251(12) \\
\text { gamma }=90\end{array}$ \\
\hline \multirow[t]{2}{*}{ Temperature: } & $100 \mathrm{~K}$ & & \\
\hline & Calculated & & Reported \\
\hline Volume & $7186.8(5)$ & & $7186.8(5)$ \\
\hline Space group & $\mathrm{P} 21 / \mathrm{n}$ & & $\mathrm{P} 21 / \mathrm{n}$ \\
\hline Hall group & $-\mathrm{P} 2 \mathrm{yn}$ & & $-\mathrm{P} 2 \mathrm{yn}$ \\
\hline Moiety formula & C76 H62 Br Co & $\mathrm{N} 5, \quad 2(\mathrm{C} 7 \mathrm{H} 8)$ & $\mathrm{C} 76 \mathrm{H} 62 \mathrm{BrCo}, 2$ (C7H8) \\
\hline Sum formula & $\mathrm{C} 90 \mathrm{H} 78 \mathrm{Br} \mathrm{Co}$ & N5 & C90 H78 Br Co N5 \\
\hline Mr & 1368.41 & & 1368.41 \\
\hline$D x, g c m-3$ & 1.265 & & 1.265 \\
\hline $\mathrm{Z}$ & 4 & & 4 \\
\hline $\mathrm{Mu} \quad(\mathrm{mm}-1)$ & 0.846 & & 0.846 \\
\hline F000 & 2860.0 & & 2860.0 \\
\hline F000' & 2861.10 & & \\
\hline$h, k, l$ max & $13,27,32$ & & $13,27,32$ \\
\hline Nref & 12707 & & 12706 \\
\hline Tmin, Tmax & $0.903,0.951$ & & $0.661,0.745$ \\
\hline Tmin' & 0.844 & & \\
\hline \multicolumn{4}{|c|}{$\begin{array}{l}\text { Correction method= \# } \\
\text { AbsCorr = MULTI-SCAN }\end{array}$} \\
\hline \multicolumn{2}{|c|}{ Data completeness $=1.000$} & \multicolumn{2}{|c|}{ Theta $(\max )=25.027$} \\
\hline \multicolumn{2}{|c|}{$R($ reflections $)=0.0860(7291)$} & \multicolumn{2}{|c|}{$\mathrm{wR} 2($ reflections $)=0.1631(12706)$} \\
\hline$S=1.091$ & Npar & $r=1086$ & \\
\hline
\end{tabular}


The following ALERTS were generated. Each ALERT has the format

test-name_ALERT_alert-type_alert-level.

Click on the hyperlinks for more details of the test.

Alert level $B$

PLAT250_ALERT_2_B Large U3/U1 Ratio for Average U(i,j) Tensor .... 4.3 Note

Author Response: We cannot avoid this alert by using SIMU only to model the disorder in the structure. We have applied a stricter restraint by lowering down the parameter from SIMU 0.04 (default) to SIMU 0.02 or even to SIMU 0.01. As SIMU 0.01 assumes the default 1.4 distance criterion, it has been applied as a last-resort solution. We note that such Alert at B level can be avoided by applying EADP for the pair of disordered atoms and/or RIGU on the bonded atoms in disoder fragment.

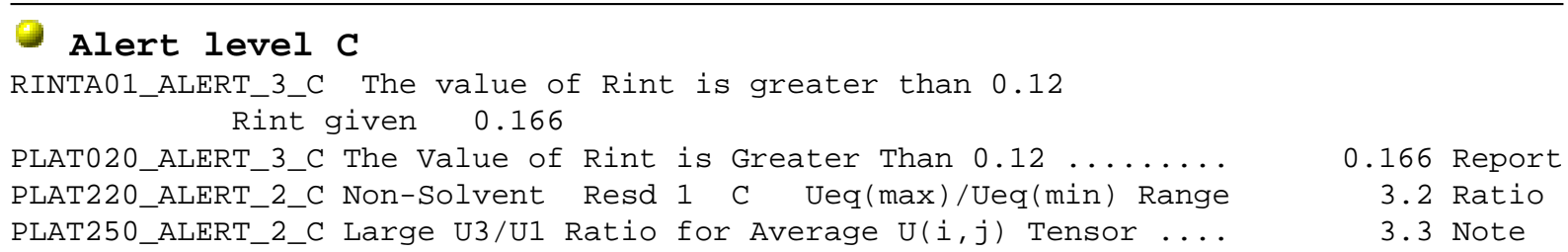

Author Response: We cannot avoid this alert by using SIMU only to model the disorder in the structure. We have applied a stricter restraint by lowering down the parameter from SIMU 0.04 (default) to SIMU 0.02 or even to SIMU 0.01. As SIMU 0.01 assumes the default 1.4 distance criterion, it has been applied as a last-resort solution. We note that such Alert at B level can be avoided by applying EADP for the pair of disordered atoms and/or RIGU on the bonded atoms in disoder fragment.

Author Response: We cannot avoid this alert by using SIMU only to model the disorder in the structure. We have applied a stricter restraint by lowering down the parameter from SIMU 0.04 (default) to SIMU 0.02 or even to SIMU 0.01. As SIMU 0.01 assumes the default 1.4 distance criterion, it has been applied as a last-resort solution. We note that such Alert at B level can be avoided by applying EADP for the pair of disordered atoms and/or RIGU on the bonded atoms in disoder fragment. 


\begin{abstract}
Author Response: We cannot avoid this alert by using SIMU only to model the disorder in the structure. We have applied a stricter restraint by lowering down the parameter from SIMU 0.04 (default) to SIMU 0.02 or even to SIMU 0.01. As SIMU 0.01 assumes the default 1.4 distance criterion, it has been applied as a last-resort solution. We note that such Alert at B level can be avoided by applying EADP for the pair of disordered atoms and/or RIGU on the bonded atoms in disoder fragment.
\end{abstract}

PLAT341_ALERT_3_C PLAT906_ALERT_3_C

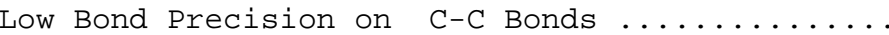
Large $K$ Value in the Analysis of Variance ...... Large $K$ Value in the Analysis of Variance ......
0.0081 Ang. 15.633 Check 3.595 Check

Alert level $\mathrm{G}$

FORMU01_ALERT_1_G There is a discrepancy between the atom counts in the

_chemical_formula_sum and_chemical_formula_moiety. This is

usually due to the moiety formula being in the wrong format.

Atom count from_chemical_formula_sum: C90 H78 Br1 Co1 N5

Atom count from_chemical_formula_moiety:C90 H78 Br1 Co1

\begin{tabular}{|c|c|c|c|c|}
\hline T002_ALERT_2_G & istance or Angle Rest & ats o & & \\
\hline PLAT003_ALERT_2_G & Number of Uiso or Uij Restrained $n$ & lon-H Atoms ... & 71 & eport \\
\hline PLAT042_ALERT_1_G & Calc. and Reported MoietyFormula S & trings Differ & Please & heck \\
\hline PLAT083_ALERT_2_G & SHELXL Second Parameter in WGHT & Jnusually Large & & Why ? \\
\hline PLAT171_ALERT_4_G & The CIF-Embedded .res File Contain & Is EADP Records & 1 & eport \\
\hline PLAT175_ALERT_4_G & The CIF-Embedded .res File & SAME Records & 1 & eport \\
\hline PLAT176_ALERT_4_G & The CIF-Embedded .res File & SADI Records & 4 & eport \\
\hline PLAT178_ALERT_4_G & The CIF-Embedded .res File Contain & SIMU Records & 6 & Report \\
\hline PLAT300_ALERT_4_G & Atom Site Occupancy of C11S & Constrained at & 0.5 & Check \\
\hline PLAT300_ALERT_4_G & Atom Site Occupancy of C12S & Constrained at & 0.5 & Check \\
\hline PLAT300_ALERT_4_G & Atom Site Occupancy of C13S & Constrained at & 0.5 & Check \\
\hline PLAT300_ALERT_4_G & Atom Site Occupancy of C14S & Constrained at & 0.5 & Check \\
\hline PLAT300_ALERT_4_G & Atom Site Occupancy of C15S & Constrained at & 0.5 & Check \\
\hline PLAT300_ALERT_4_G & Atom Site Occupancy of C16S & Constrained at & 0.5 & Check \\
\hline PLAT300_ALERT_4_G & Atom Site Occupancy of C17S & Constrained at & 0.5 & Check \\
\hline PLAT300_ALERT_4_G & Atom Site Occupancy of H11S & Constrained at & 0.5 & Check \\
\hline PLAT300_ALERT_4_G & Atom Site Occupancy of H12S & Constrained at & 0.5 & Check \\
\hline PLAT300_ALERT_4_G & Atom Site Occupancy of H13S & Constrained at & 0.5 & Check \\
\hline PLAT300_ALERT_4_G & Atom Site Occupancy of H14S & Constrained at & 0.5 & Check \\
\hline PLAT300_ALERT_4_G & Atom Site Occupancy of H15S & Constrained at & 0.5 & Check \\
\hline PLAT300_ALERT_4_G & Atom Site Occupancy of H17A & Constrained at & 0.5 & Check \\
\hline PLAT300_ALERT_4_G & Atom Site Occupancy of H17B & Constrained at & 0.5 & Check \\
\hline PLAT300_ALERT_4_G & Atom Site Occupancy of H17C & Constrained at & 0.5 & Check \\
\hline PLAT300_ALERT_4_G & Atom Site Occupancy of C21S & Constrained at & 0.5 & Check \\
\hline PLAT300_ALERT_4_G & Atom Site Occupancy of C22S & Constrained at & 0.5 & Check \\
\hline PLAT300_ALERT_4_G & Occupancy & Constrained at & 0.5 & Check \\
\hline PLAT300_ALERT_4_G & Atom Site Occupancy of C24S & Constrained at & 0.5 & Check \\
\hline PLAT300_ALERT_4_G & Atom Site Occupancy of C25S & Constrained at & 0.5 & Check \\
\hline PLAT300_ALERT_4_G & Atom Site Occupancy of C26S & Constrained at & 0.5 & Check \\
\hline PLAT300_ALERT_4_G & Atom Site Occupancy of C27S & Constrained at & 0.5 & Check \\
\hline PLAT300_ALERT_4_G & Atom Site Occupancy of H21S & Constrained at & 0.5 & Check \\
\hline PLAT300_ALERT_4_G & Atom Site Occupancy of $\mathrm{H} 22 \mathrm{~S}$ & Constrained at & 0.5 & Check \\
\hline PLAT300_ALERT_4_G & Atom Site Occupancy of H23S & Constrained at & 0.5 & Check \\
\hline PLAT300_ALERT_4_G & Atom Site Occupancy of $\mathrm{H} 24 \mathrm{~S}$ & Constrained at & 0.5 & Check \\
\hline PLAT300_ALERT_4_G & Atom Site Occupancy of H25S & Constrained at & 0.5 & Check \\
\hline PLAT300_ALERT_4_G & Atom Site Occupancy of H27A & Constrained at & 0.5 & Check \\
\hline PLAT300_ALERT_4_G & Atom Site Occupancy of $\mathrm{H} 27 \mathrm{~B}$ & Constrained at & 0.5 & Check \\
\hline PLAT300_ALERT_4_G & Atom Site Occupancy of $\mathrm{H} 27 \mathrm{C}$ & Constrained at & 0.5 & Check \\
\hline PLAT301_ALERT_3_G & Main Residue Disorder .... & $\ldots(\operatorname{Resd} 1)$ & $27 \%$ & Note \\
\hline PLAT302_ALERT_4_G & Anion/Solvent/Minor-Residue & der (Resd 2 & $100 \%$ & Note \\
\hline
\end{tabular}




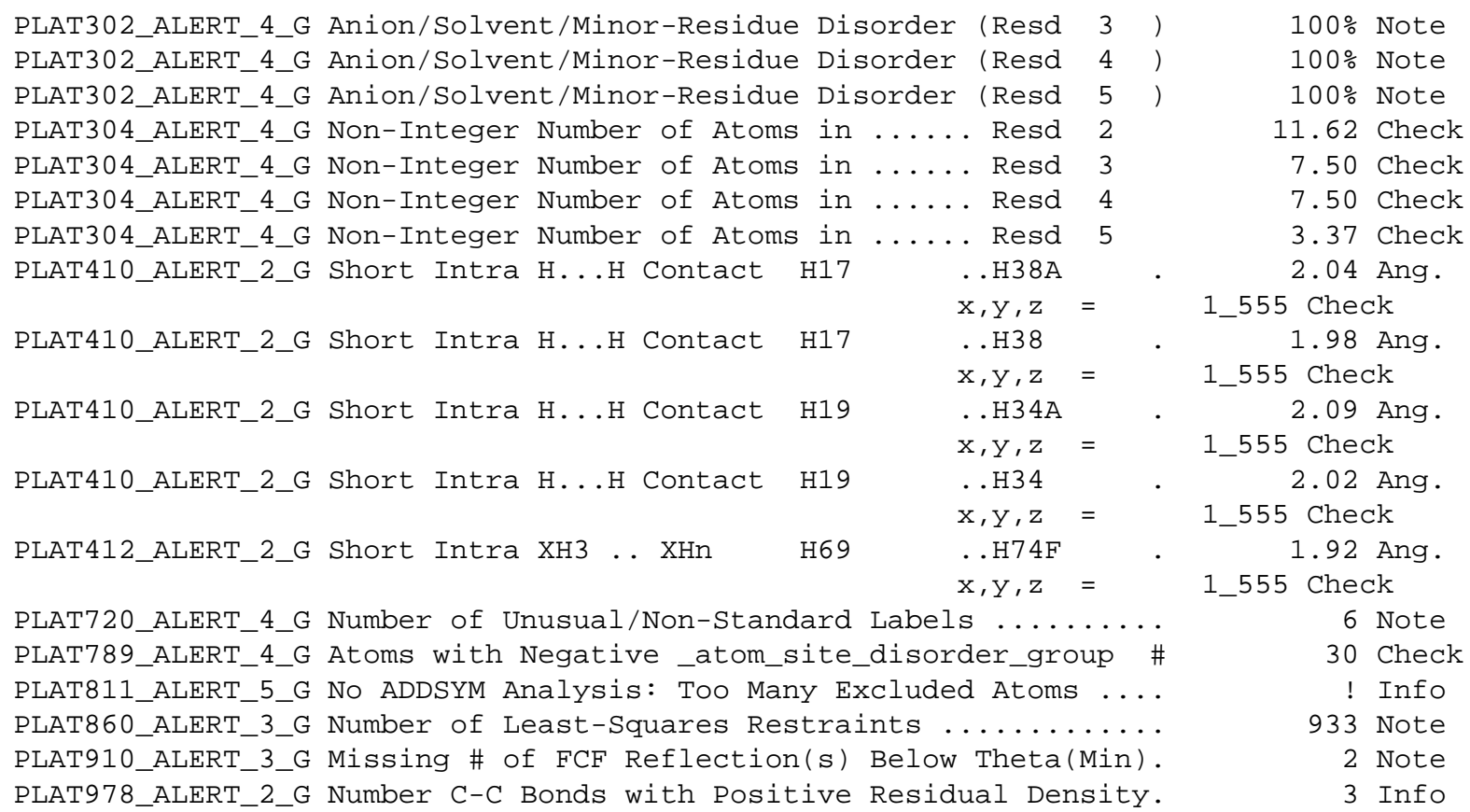

0 ALERT level $\mathbf{A}=$ Most likely a serious problem - resolve or explain

1 ALERT level $\mathbf{B}=\mathrm{A}$ potentially serious problem, consider carefully

9 ALERT level $\mathbf{C}=$ Check. Ensure it is not caused by an omission or oversight

59 ALERT level $\mathbf{G}=$ General information/check it is not something unexpected

2 ALERT type 1 CIF construction/syntax error, inconsistent or missing data

14 ALERT type 2 Indicator that the structure model may be wrong or deficient

8 ALERT type 3 Indicator that the structure quality may be low

44 ALERT type 4 Improvement, methodology, query or suggestion

1 ALERT type 5 Informative message, check 
It is advisable to attempt to resolve as many as possible of the alerts in all categories. Often the minor alerts point to easily fixed oversights, errors and omissions in your CIF or refinement strategy, so attention to these fine details can be worthwhile. In order to resolve some of the more serious problems it may be necessary to carry out additional measurements or structure refinements. However, the purpose of your study may justify the reported deviations and the more serious of these should normally be commented upon in the discussion or experimental section of a paper or in the "special_details" fields of the CIF. checkCIF was carefully designed to identify outliers and unusual parameters, but every test has its limitations and alerts that are not important in a particular case may appear. Conversely, the absence of alerts does not guarantee there are no aspects of the results needing attention. It is up to the individual to critically assess their own results and, if necessary, seek expert advice.

\section{Publication of your CIF in IUCr journals}

A basic structural check has been run on your CIF. These basic checks will be run on all CIFs submitted for publication in IUCr journals (Acta Crystallographica, Journal of Applied Crystallography, Journal of Synchrotron Radiation); however, if you intend to submit to Acta Crystallographica Section C or E or IUCrData, you should make sure that full publication checks are run on the final version of your CIF prior to submission.

\section{Publication of your CIF in other journals}

Please refer to the Notes for Authors of the relevant journal for any special instructions relating to CIF submission.

\section{PLATON version of 07/08/2019; check.def file version of 30/07/2019}


Datablock 3_ArLCoBrN3Ar - ellipsoid plot

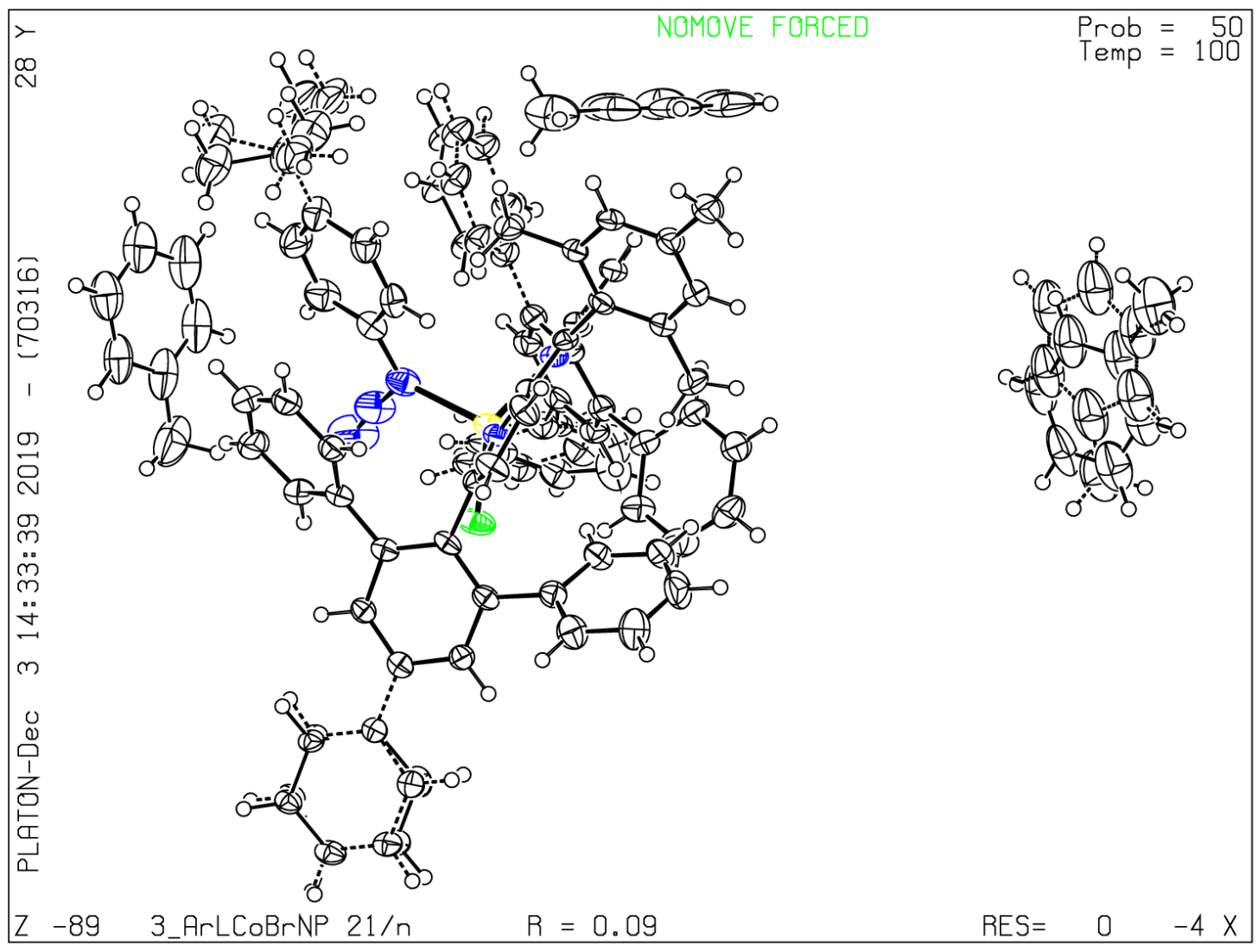




\section{checkCIF/PLATON report}

Structure factors have been supplied for datablock(s) 4_ArLCoBrannulation

THIS REPORT IS FOR GUIDANCE ONLY. IF USED AS PART OF A REVIEW PROCEDURE FOR PUBLICATION, IT SHOULD NOT REPLACE THE EXPERTISE OF AN EXPERIENCED CRYSTALLOGRAPHIC REFEREE.

No syntax errors found. CIF dictionary Interpreting this report

\section{Datablock: 4_ArLCoBrannulation}

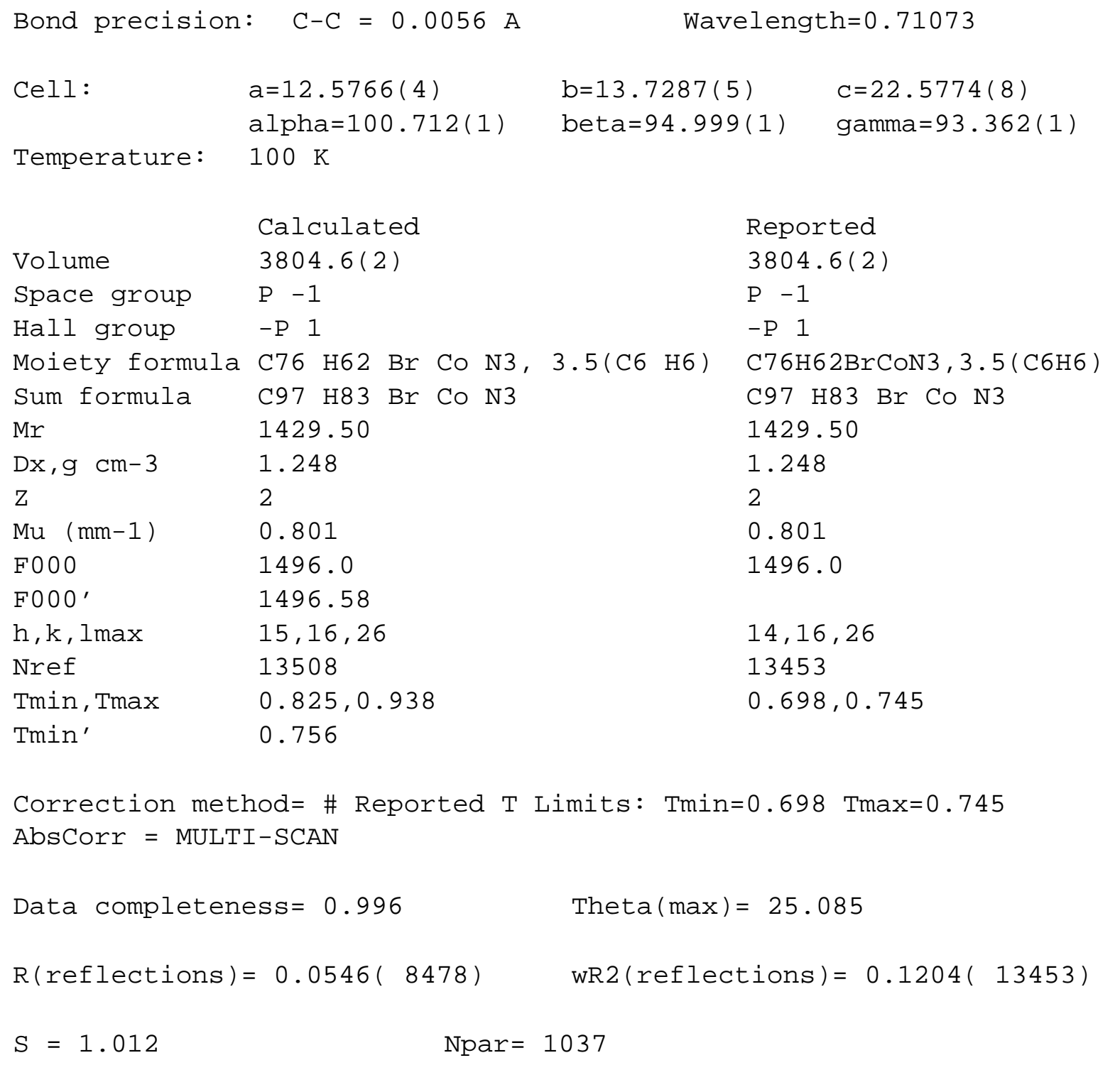


Alert level $\mathrm{C}$

PLAT220 ALERT 2 C Non-Solvent Resd 1 C Ueq (max)/Ueg(min) Range PLAT222_ALERT_3_C Non-Solv. Resd 1 H Uiso(max)/Uiso(min) Range PLAT250_ALERT_2_C Large U3/U1 Ratio for Average U(i,j) Tensor .... PLAT250_ALERT_2_C Large U3/U1 Ratio for Average U(i,j) Tensor .... PLAT420_ALERT_2_C D-H Without Acceptor N3 PLAT906_ALERT_3_C Large $K$ Value in the Analysis of Variance ...... PLAT910_ALERT_3_C Missing \# of FCF Reflection(s) Below Theta(Min). PLAT911_ALERT_3_C Missing FCF Refl Between Thmin \& STh/L= 0.597

3.5 Ratio

4.2 Ratio

2.8 Note

2.1 Note

Please Check

3.679 Check

10 Note

45 Report

\section{Alert level $\mathbf{G}$}

PLAT002_ALERT_2_G Number of Distance or Angle Restraints on AtSite PLAT003_ALERT_2_G Number of Uiso or Uij Restrained non-H Atoms ... PLAT042_ALERT_1_G Calc. and Reported MoietyFormula Strings Differ PLAT154_ALERT_1_G The s.u.'s on the Cell Angles are Equal .. (Note) PLAT176_ALERT_4_G The CIF-Embedded.res File Contains SADI Records PLAT178_ALERT_4_G The CIF-Embedded.res File Contains SIMU Records PLAT301_ALERT_3_G Main Residue Disorder..................... 1 ) PLAT302_ALERT_4_G Anion/Solvent/Minor-Residue Disorder (Resd 4) PLAT302_ALERT_4_G Anion/Solvent/Minor-Residue Disorder (Resd 5 ) PLAT302_ALERT_4_G Anion/Solvent/Minor-Residue Disorder (Resd 6) PLAT302_ALERT_4_G Anion/Solvent/Minor-Residue Disorder (Resd 7 ) PLAT304_ALERT_4_G Non-Integer Number of Atoms in ..... Resd 4 PLAT304_ALERT_4_G Non-Integer Number of Atoms in ..... Resd 5 PLAT304_ALERT_4_G Non-Integer Number of Atoms in ..... Resd 6 PLAT304_ALERT_4_G Non-Integer Number of Atoms in ..... Resd 7 PLAT432_ALERT_2_G Short Inter X...Y Contact C54 ..C22T

$$
2-\mathrm{x}, 1-\mathrm{y}, 1-\mathrm{z}=
$$

PLAT789_ALERT_4_G Atoms with Negative _atom_site_disorder_group \# PLAT793_ALERT_4_G Model has Chirality at N3 (Centro SPGR) PLAT793_ALERT_4_G Model has Chirality at C47 (Centro SPGR) PLAT793_ALERT_4_G Model has Chirality at C48 (Centro SPGR) PLAT860_ALERT_3_G Number of Least-Squares Restraints .......... PLAT933_ALERT_2_G Number of OMIT Records in Embedded.res File... PLAT978_ALERT_2_G Number C-C Bonds with Positive Residual Density.

3 Note

36 Report Please Check

0.001 Degree

1 Report

3 Report

7\% Note

$100 \%$ Note

$100 \%$ Note

$100 \%$ Note

$100 \%$ Note

7.69 Check

4.31 Check

3. 01 Check

2.99 Check

$3.15 \mathrm{Ang}$. 2_766 Check

24 Check

S Verify

$S$ Verify

$S$ Verify

481 Note

3 Note

1 Info

0 ALERT level $\mathbf{A}=$ Most likely a serious problem - resolve or explain

0 ALERT level $\mathbf{B}=\mathrm{A}$ potentially serious problem, consider carefully

8 ALERT level $\mathbf{C}=$ Check. Ensure it is not caused by an omission or oversight

23 ALERT level $\mathbf{G}=$ General information/check it is not something unexpected

2 ALERT type 1 CIF construction/syntax error, inconsistent or missing data

9 ALERT type 2 Indicator that the structure model may be wrong or deficient

6 ALERT type 3 Indicator that the structure quality may be low

14 ALERT type 4 Improvement, methodology, query or suggestion

0 ALERT type 5 Informative message, check 
It is advisable to attempt to resolve as many as possible of the alerts in all categories. Often the minor alerts point to easily fixed oversights, errors and omissions in your CIF or refinement strategy, so attention to these fine details can be worthwhile. In order to resolve some of the more serious problems it may be necessary to carry out additional measurements or structure refinements. However, the purpose of your study may justify the reported deviations and the more serious of these should normally be commented upon in the discussion or experimental section of a paper or in the "special_details" fields of the CIF. checkCIF was carefully designed to identify outliers and unusual parameters, but every test has its limitations and alerts that are not important in a particular case may appear. Conversely, the absence of alerts does not guarantee there are no aspects of the results needing attention. It is up to the individual to critically assess their own results and, if necessary, seek expert advice.

\section{Publication of your CIF in IUCr journals}

A basic structural check has been run on your CIF. These basic checks will be run on all CIFs submitted for publication in IUCr journals (Acta Crystallographica, Journal of Applied Crystallography, Journal of Synchrotron Radiation); however, if you intend to submit to Acta Crystallographica Section C or E or IUCrData, you should make sure that full publication checks are run on the final version of your CIF prior to submission.

\section{Publication of your CIF in other journals}

Please refer to the Notes for Authors of the relevant journal for any special instructions relating to CIF submission.

\section{PLATON version of 07/08/2019; check.def file version of 30/07/2019}


Datablock 4_ArLCoBrannulation - ellipsoid plot

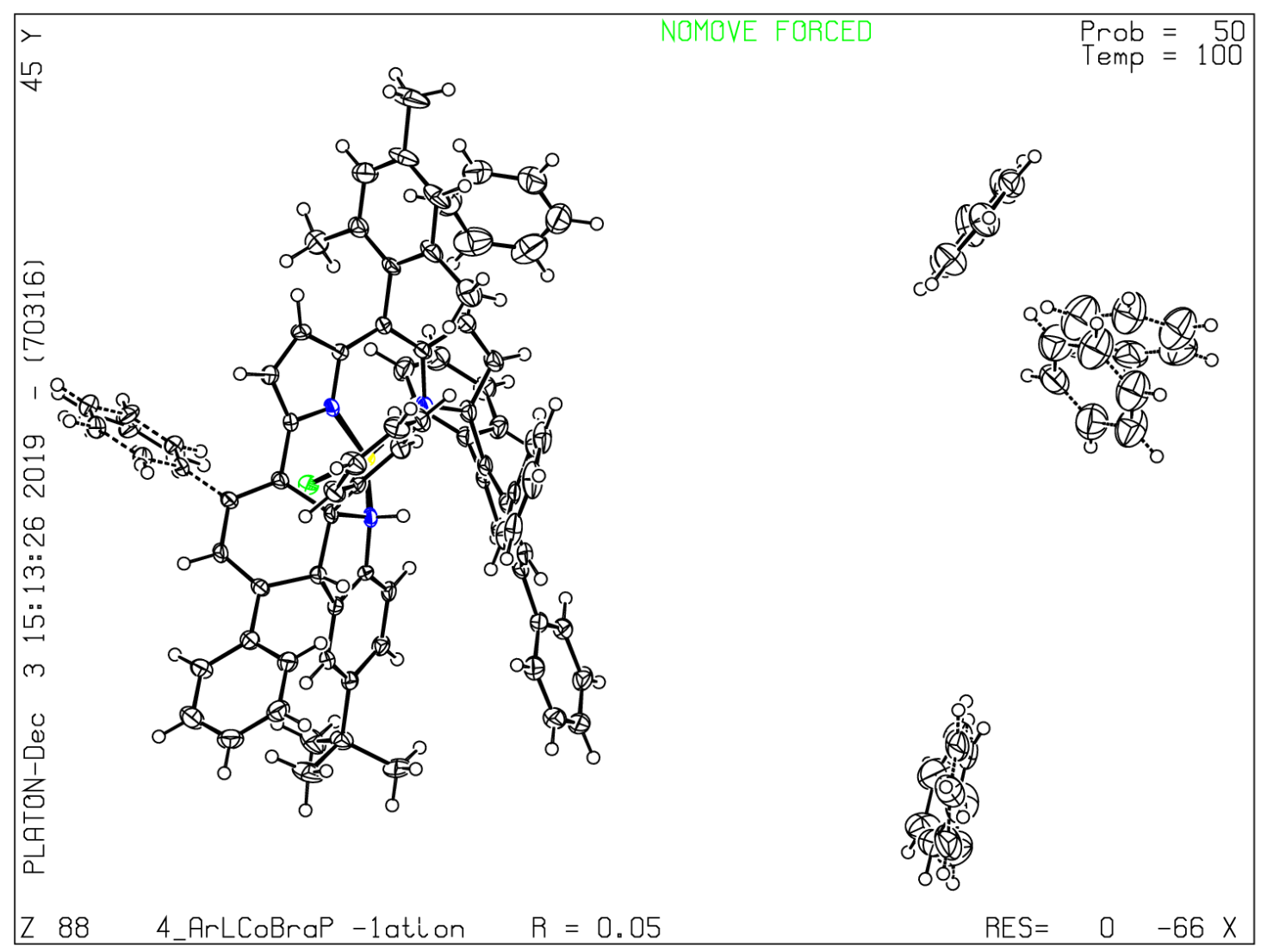




\section{checkCIF/PLATON report}

Structure factors have been supplied for datablock(s) 5_ArLCoBrNH2Ar

THIS REPORT IS FOR GUIDANCE ONLY. IF USED AS PART OF A REVIEW PROCEDURE FOR PUBLICATION, IT SHOULD NOT REPLACE THE EXPERTISE OF AN EXPERIENCED CRYSTALLOGRAPHIC REFEREE.

No syntax errors found. CIF dictionary Interpreting this report

\section{Datablock: 5_ArLCoBrNH2Ar}

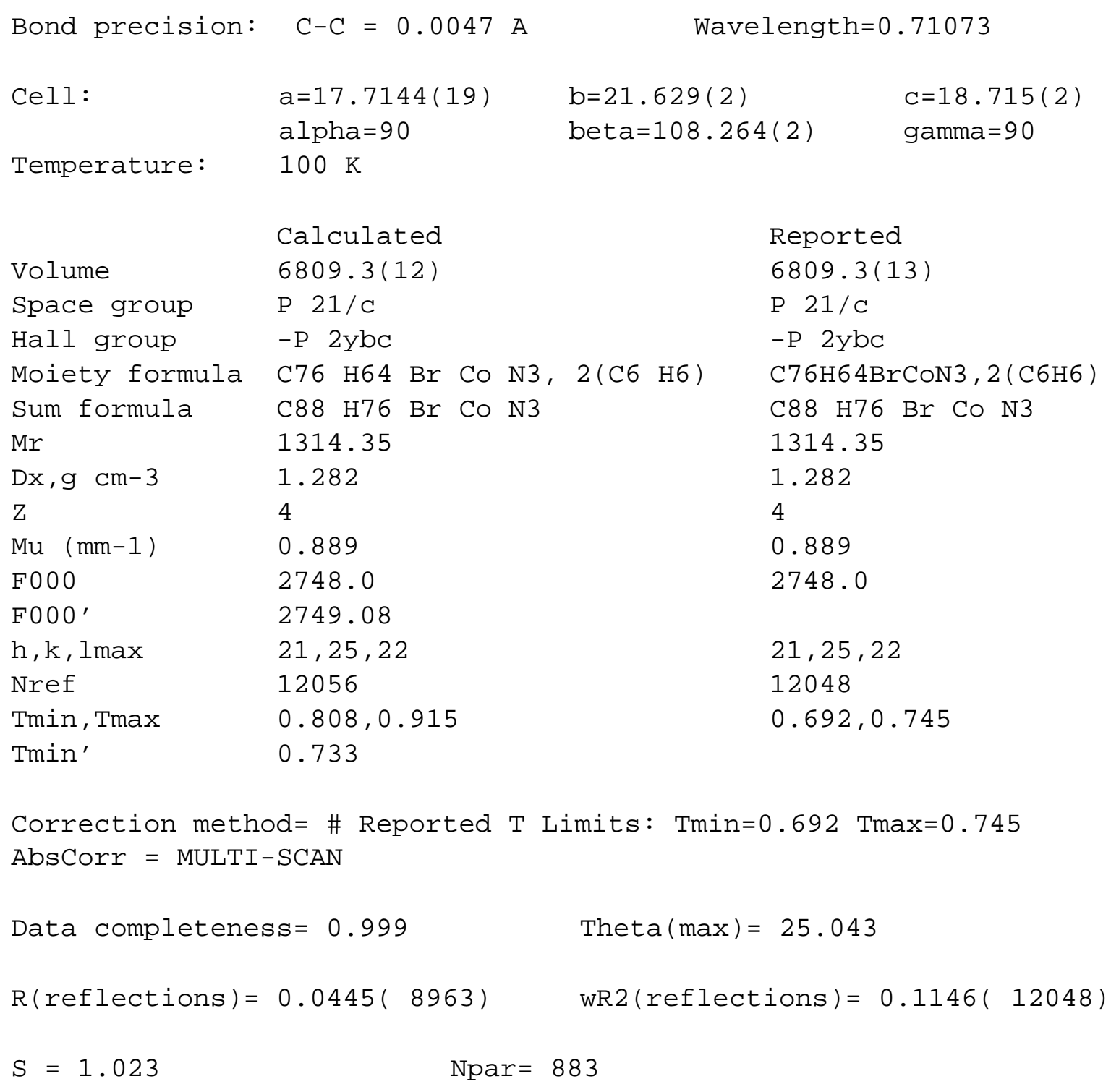


Alert level $\mathrm{C}$

CRYSC01_ALERT_1_C The word below has not been recognised as a standard identifier.

maroon

CRYSC01_ALERT_1_C No recognised colour has been given for crystal colour.

PLAT094_ALERT_2_C Ratio of Maximum / Minimum Residual Density ....

PLAT220_ALERT_2_C Non-Solvent Resd 1 C Ueq(max)/Ueq(min) Range

3.69 Report

PLAT250_ALERT_2_C Large U3/U1 Ratio for Average U(i,j) Tensor ....

3.5 Ratio

PLAT250 ALERT 2 C Large U3/U1 Ratio for Average U(i,j) Tensor .....

PLAT331_ALERT_2_C Small Aver Phenyl C-C Dist C1S -

PLAT420_ALERT_2_C D-H Without Acceptor N3

PLAT420_ALERT_2_C D-H Without Acceptor N3

PLAT905_ALERT_3_C Negative $K$ value in the Analysis of Variance ...

PLAT911_ALERT_3_C Missing FCF Refl Between Thmin \& STh/L= 0.596

2.9 Note

2.6 Note

1.36 Ang.

Please Check

Please Check

-0.066 Report

7 Report

\section{Alert level G}

PLAT003_ALERT_2_G Number of Uiso or Uij Restrained non-H Atoms ...

PLAT042_ALERT_1_G Calc. and Reported MoietyFormula Strings Differ

PLAT083_ALERT_2_G SHELXL Second Parameter in WGHT Unusually Large

PLAT178_ALERT_4_G The CIF-Embedded.res File Contains SIMU Records

PLAT302_ALERT_4_G Anion/Solvent/Minor-Residue Disorder (Resd 3 )

PLAT302_ALERT_4_G Anion/Solvent/Minor-Residue Disorder (Resd 4)

PLAT304_ALERT_4_G Non-Integer Number of Atoms in ..... Resd 3

PLAT304_ALERT_4_G Non-Integer Number of Atoms in ..... Resd 4

PLAT860_ALERT_3_G Number of Least-Squares Restraints ..........

PLAT909_ALERT_3_G Percentage of I>2sig(I) Data at Theta(Max) Still

PLAT910_ALERT_3_G Missing \# of FCF Reflection(s) Below Theta(Min).

PLAT978_ALERT_2_G Number C-C Bonds with Positive Residual Density.
12 Report
Please Check
7.21 Why ?
1 Report
$100 \%$ Note
$100 \%$ Note
8.35 Check
3. 65 Check
192 Note
$51 \%$ Note
1 Note
4 Info

0 ALERT level $\mathbf{A}=$ Most likely a serious problem - resolve or explain

0 ALERT level $\mathbf{B}=\mathrm{A}$ potentially serious problem, consider carefully

11 ALERT level $\mathbf{C}=$ Check. Ensure it is not caused by an omission or oversight

12 ALERT level $\mathbf{G}=$ General information/check it is not something unexpected

3 ALERT type 1 CIF construction/syntax error, inconsistent or missing data

10 ALERT type 2 Indicator that the structure model may be wrong or deficient

5 ALERT type 3 Indicator that the structure quality may be low

5 ALERT type 4 Improvement, methodology, query or suggestion

0 ALERT type 5 Informative message, check 
It is advisable to attempt to resolve as many as possible of the alerts in all categories. Often the minor alerts point to easily fixed oversights, errors and omissions in your CIF or refinement strategy, so attention to these fine details can be worthwhile. In order to resolve some of the more serious problems it may be necessary to carry out additional measurements or structure refinements. However, the purpose of your study may justify the reported deviations and the more serious of these should normally be commented upon in the discussion or experimental section of a paper or in the "special_details" fields of the CIF. checkCIF was carefully designed to identify outliers and unusual parameters, but every test has its limitations and alerts that are not important in a particular case may appear. Conversely, the absence of alerts does not guarantee there are no aspects of the results needing attention. It is up to the individual to critically assess their own results and, if necessary, seek expert advice.

\section{Publication of your CIF in IUCr journals}

A basic structural check has been run on your CIF. These basic checks will be run on all CIFs submitted for publication in IUCr journals (Acta Crystallographica, Journal of Applied Crystallography, Journal of Synchrotron Radiation); however, if you intend to submit to Acta Crystallographica Section C or E or IUCrData, you should make sure that full publication checks are run on the final version of your CIF prior to submission.

\section{Publication of your CIF in other journals}

Please refer to the Notes for Authors of the relevant journal for any special instructions relating to CIF submission.

\section{PLATON version of 07/08/2019; check.def file version of 30/07/2019}


Datablock 5_ArLCoBrNH2Ar - ellipsoid plot

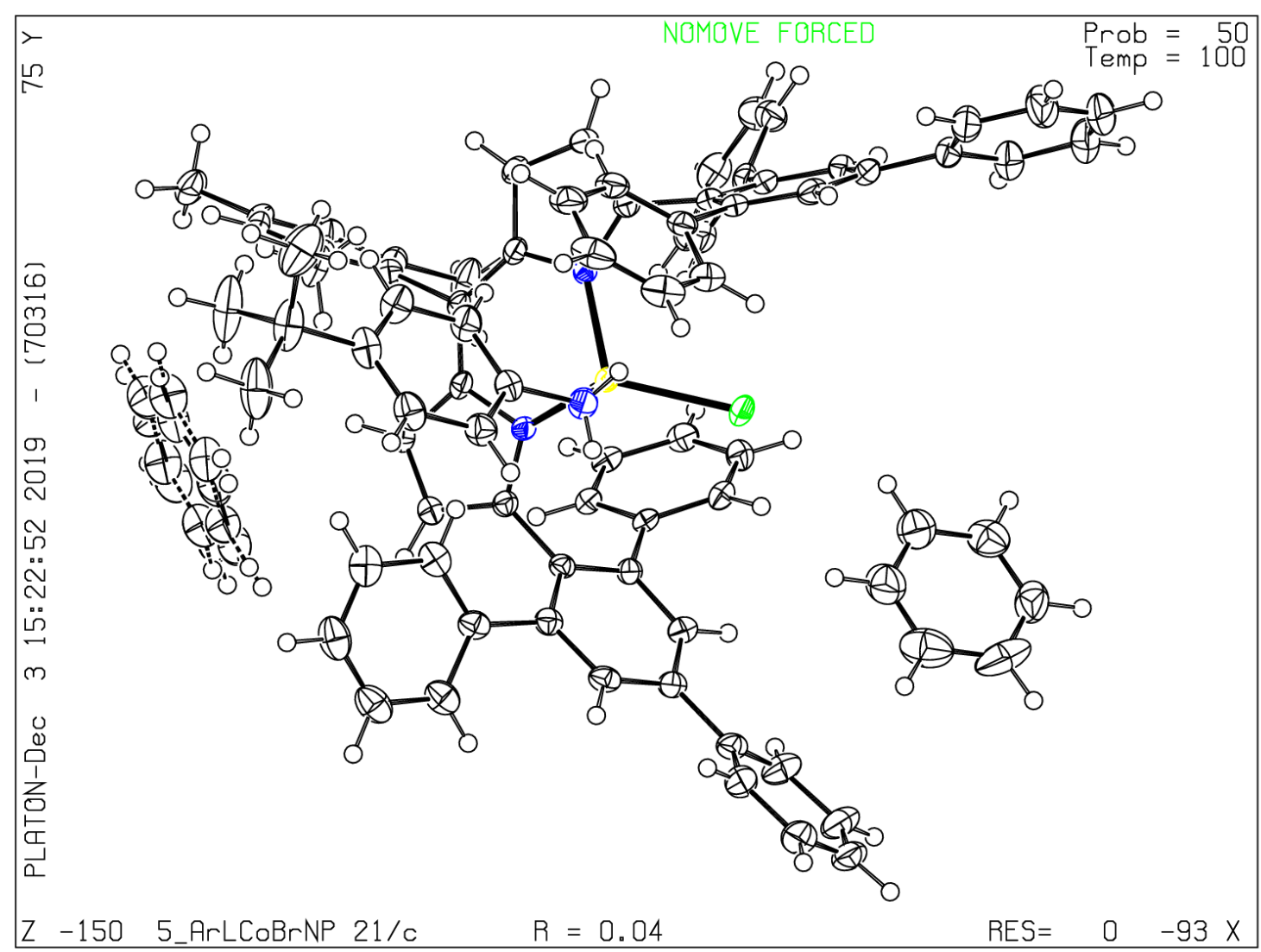




\section{checkCIF/PLATON report}

Structure factors have been supplied for datablock(s) 7_ArLCoBrN3R_1

THIS REPORT IS FOR GUIDANCE ONLY. IF USED AS PART OF A REVIEW PROCEDURE FOR PUBLICATION, IT SHOULD NOT REPLACE THE EXPERTISE OF AN EXPERIENCED CRYSTALLOGRAPHIC REFEREE.

No syntax errors found. CIF dictionary Interpreting this report

\section{Datablock: 7_ArLCoBrN3R_1}

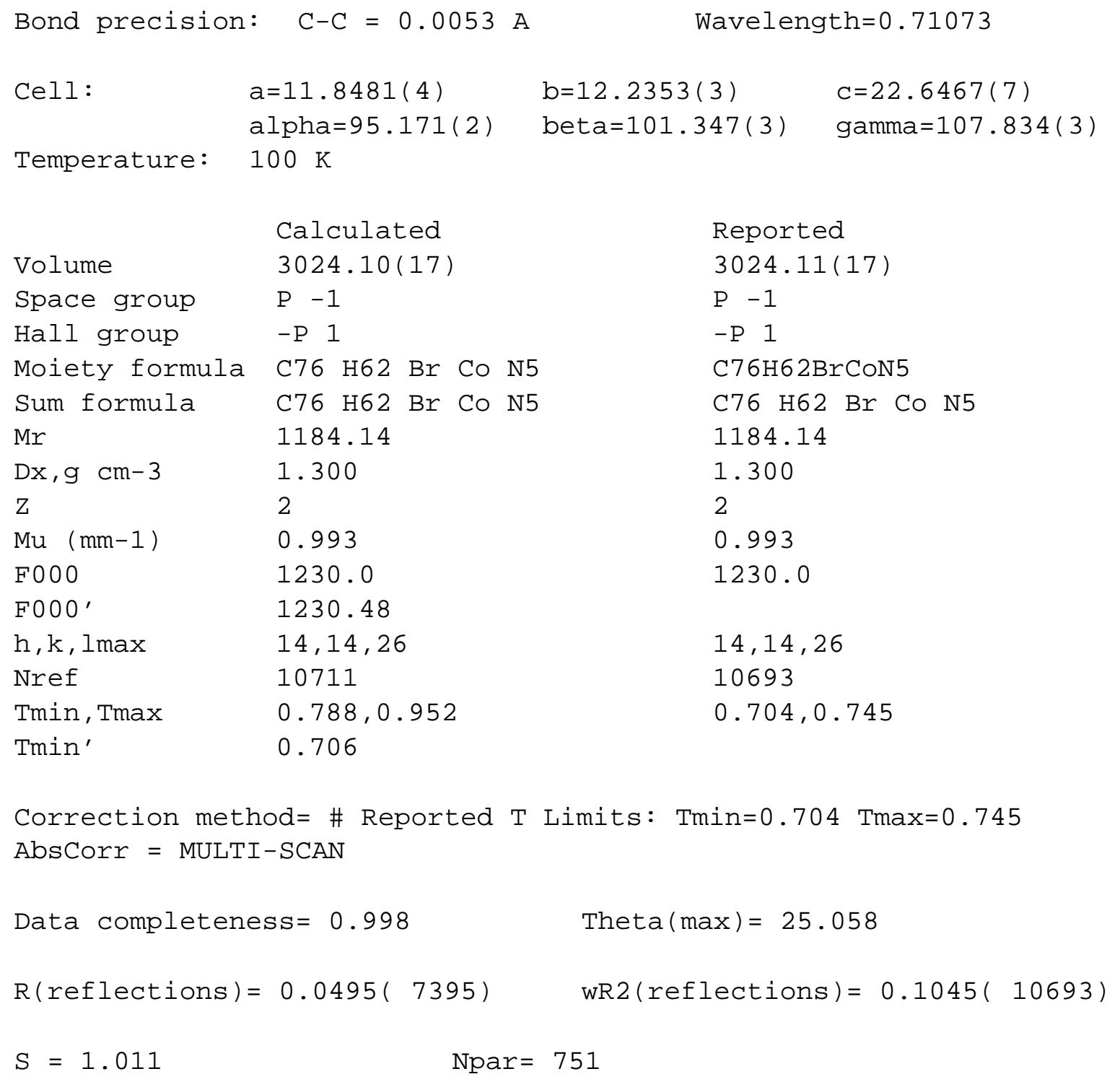


The following ALERTS were generated. Each ALERT has the format

test-name_ALERT_alert-type_alert-level.

Click on the hyperlinks for more details of the test.

Alert level $B$

PLAT910_ALERT_3_B Missing \# of FCF Reflection(s) Below Theta(Min). 13 Note

\section{Author Response: Reflections were blocked by the beamstop}

\section{Alert level $\mathrm{C}$}

PLAT220_ALERT_2_C Non-Solvent Resd 1 C Ueq(max)/Ueq(min) Range

PLAT230_ALERT_2_C Hirshfeld Test Diff for N3 -

3.3 Ratio

PLAT906_ALERT_3_C Large $K$ Value in the Analysis of Variance ......

PLAT911_ALERT_3_C Missing FCF Refl Between Thmin \& STh/L= 0.596

$6.3 \mathrm{~s} . \mathrm{u}$.

2. 648 Check

6 Report

Alert level G

PLAT042_ALERT_1_G Calc. and Reported MoietyFormula Strings Differ Please Check

PLAT232_ALERT_2_G Hirshfeld Test Diff (M-X) Br1 --Co1 10.0 s.u.

PLAT380_ALERT_4_G Incorrectly? Oriented X(sp2)-Methyl Moiety ...... C9 Check

PLAT909_ALERT_3_G Percentage of I>2sig(I) Data at Theta (Max) Still 42\% Note

PLAT933_ALERT_2_G Number of OMIT Records in Embedded.res File... 6 Note

PLAT978_ALERT_2_G Number C-C Bonds with Positive Residual Density. 1 Info

0 ALERT level $\mathbf{A}=$ Most likely a serious problem - resolve or explain

1 ALERT level B = A potentially serious problem, consider carefully

4 ALERT level $\mathbf{C}=$ Check. Ensure it is not caused by an omission or oversight

6 ALERT level $\mathbf{G}=$ General information/check it is not something unexpected

1 ALERT type 1 CIF construction/syntax error, inconsistent or missing data

5 ALERT type 2 Indicator that the structure model may be wrong or deficient

4 ALERT type 3 Indicator that the structure quality may be low

1 ALERT type 4 Improvement, methodology, query or suggestion

0 ALERT type 5 Informative message, check 
It is advisable to attempt to resolve as many as possible of the alerts in all categories. Often the minor alerts point to easily fixed oversights, errors and omissions in your CIF or refinement strategy, so attention to these fine details can be worthwhile. In order to resolve some of the more serious problems it may be necessary to carry out additional measurements or structure refinements. However, the purpose of your study may justify the reported deviations and the more serious of these should normally be commented upon in the discussion or experimental section of a paper or in the "special_details" fields of the CIF. checkCIF was carefully designed to identify outliers and unusual parameters, but every test has its limitations and alerts that are not important in a particular case may appear. Conversely, the absence of alerts does not guarantee there are no aspects of the results needing attention. It is up to the individual to critically assess their own results and, if necessary, seek expert advice.

\section{Publication of your CIF in IUCr journals}

A basic structural check has been run on your CIF. These basic checks will be run on all CIFs submitted for publication in IUCr journals (Acta Crystallographica, Journal of Applied Crystallography, Journal of Synchrotron Radiation); however, if you intend to submit to Acta Crystallographica Section C or E or IUCrData, you should make sure that full publication checks are run on the final version of your CIF prior to submission.

\section{Publication of your CIF in other journals}

Please refer to the Notes for Authors of the relevant journal for any special instructions relating to CIF submission.

\section{PLATON version of 07/08/2019; check.def file version of 30/07/2019}


Datablock 7_ArLCoBrN3R_1 - ellipsoid plot

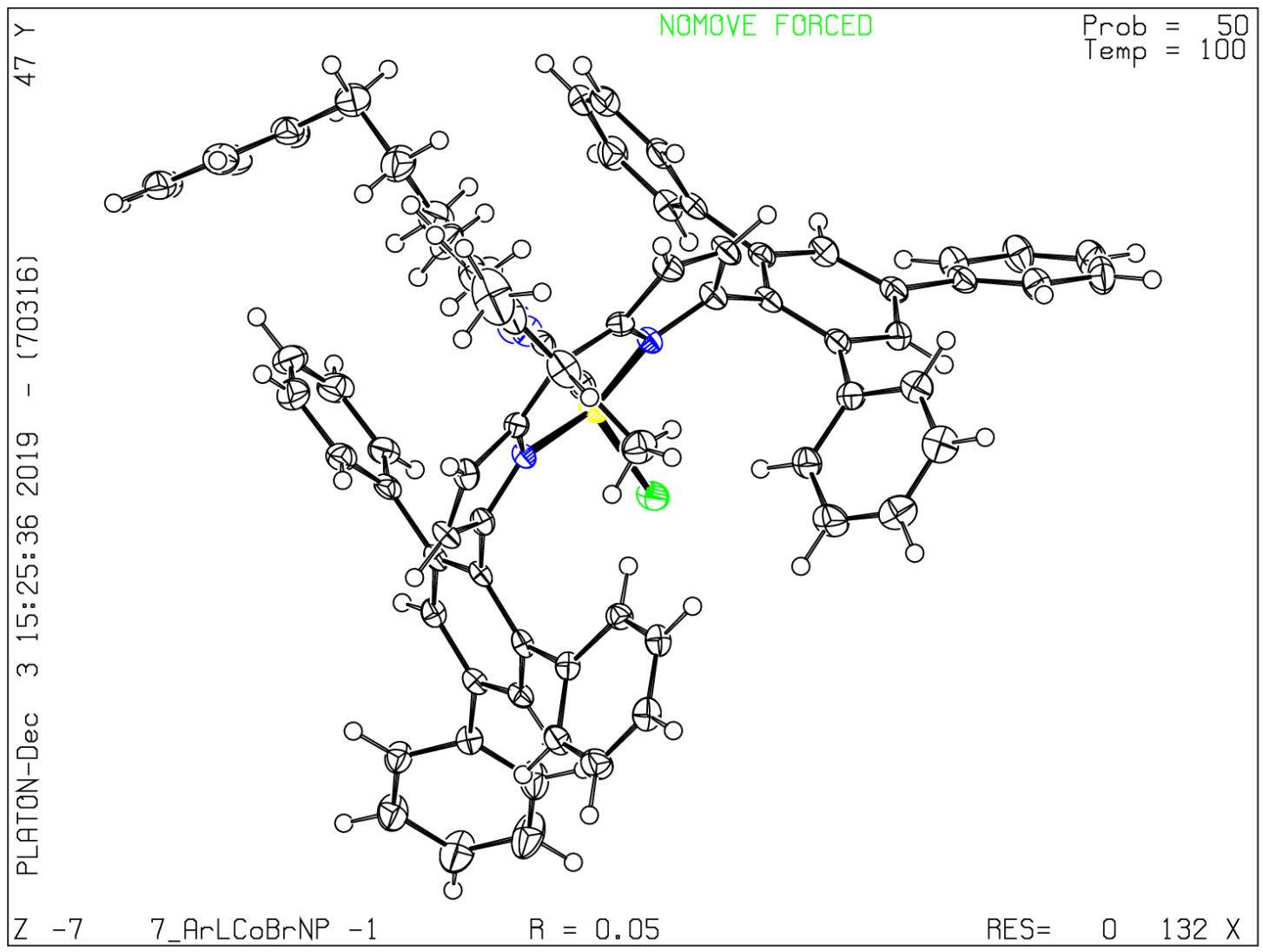




\section{checkCIF/PLATON report}

Structure factors have been supplied for datablock(s) 8_ArLCoBrN3R2-initial

THIS REPORT IS FOR GUIDANCE ONLY. IF USED AS PART OF A REVIEW PROCEDURE FOR PUBLICATION, IT SHOULD NOT REPLACE THE EXPERTISE OF AN EXPERIENCED CRYSTALLOGRAPHIC REFEREE.

No syntax errors found. CIF dictionary Interpreting this report

\section{Datablock: 8_ArLCoBrN3R2-initial}

\begin{tabular}{|c|c|c|c|}
\hline Bond precision: & $C-C=0.0069 \mathrm{~A}$ & \multicolumn{2}{|c|}{ Wavelength $=0.71073$} \\
\hline Cell: & $\begin{array}{l}a=11.5036(5) \\
a l p h a=90\end{array}$ & $\begin{array}{l}b=21.4756(11) \\
\text { bet } a=99.4205(\end{array}$ & $\begin{array}{l}\mathrm{C}=25.3211(13) \\
\text { gamma }=90\end{array}$ \\
\hline \multirow[t]{2}{*}{ Temperature: } & $100 \mathrm{~K}$ & & \\
\hline & Calculated & & Reported \\
\hline Volume & $6171.1(5)$ & & $6171.1(5)$ \\
\hline Space group & $\mathrm{P} 21 / \mathrm{C}$ & & $\mathrm{P} 21 / \mathrm{C}$ \\
\hline Hall group & $-\mathrm{P} 2 \mathrm{ybc}$ & & $-\mathrm{P} \quad 2 \mathrm{ybc}$ \\
\hline Moiety formula & C72 H62 Br Co N5, & 0.5 ( C6 H14) & $\mathrm{C} 72 \mathrm{H} 62 \mathrm{BrCON} 5,0.5(\mathrm{C} 6 \mathrm{H} 14)$ \\
\hline Sum formula & C75 H69 Br Co N5 & & C75 H69 Br Co N5 \\
\hline $\mathrm{Mr}$ & 1179.19 & & 1179.19 \\
\hline $\mathrm{Dx}, \mathrm{gcm}-3$ & 1.269 & & 1.269 \\
\hline $\mathrm{Z}$ & 4 & & 4 \\
\hline $\mathrm{Mu} \quad(\mathrm{mm}-1)$ & 0.973 & & 0.973 \\
\hline F000 & 2464.0 & & 2464.0 \\
\hline F000' & 2464.96 & & \\
\hline$h, k, l \max$ & $13,25,30$ & & $13,25,30$ \\
\hline Nref & 10995 & & 10930 \\
\hline $\operatorname{Tmin}, \operatorname{Tmax}$ & $0.749,0.856$ & & $0.726,0.801$ \\
\hline Tmin' & 0.685 & & \\
\hline \multicolumn{4}{|c|}{$\begin{array}{l}\text { Correction method= \# } \\
\text { AbsCorr = MULTI-SCAN }\end{array}$} \\
\hline \multicolumn{2}{|c|}{ Data completeness $=0.994$} & \multicolumn{2}{|c|}{ Theta $(\max )=25.096$} \\
\hline \multicolumn{2}{|c|}{$R($ reflections $)=0.0604(6924)$} & \multicolumn{2}{|c|}{$\mathrm{wR} 2($ reflections $)=0.1713(10930)$} \\
\hline$S=1.017$ & Npar $=$ & $=790$ & \\
\hline
\end{tabular}

The following ALERTS were generated. Each ALERT has the format test-name_ALERT_alert-type_alert-level.

Click on the hyperlinks for more details of the test. 
Alert level C

PLAT220_ALERT_2_C Non-Solvent Resd 1 C Ueq (max)/Ueq(min) Range PLAT230_ALERT_2_C

PLAT242_ALERT_2_C PLAT250_ALERT_2_C

PLAT250_ALERT_2_C

PLAT341_ALERT_3_C PLAT411_ALERT_2_C

PLAT906_ALERT_3_C PLAT911_ALERT_3_C
Hirshfeld Test Diff for N3 $--\mathrm{N} 4$

Low

'MainMol' Ueq as Compared to Neighbors of Large U3/U1 Ratio for Average U(i,j) Tensor .... Large U3/U1 Ratio for Average U(i,j) Tensor ....

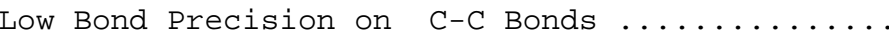
Short Inter H...H Contact H17 $1-\mathrm{x}, 1-\mathrm{y}, 1-\mathrm{z}$ Large $\mathrm{K}$ Value in the Analysis of Variance Missing FCF Refl Between Thmin \& STh/L=
$=$
3.3 Ratio

$7.0 \mathrm{~s} . \mathrm{u}$.

C73 Check

2.8 Note

2.8 Note

0.00695 Ang.

2.10 Ang.

3_666 Check

4.788 Check

59 Report

Alert level $\mathbf{G}$

PLATO02_ALERT_2_G Number of Distance or Angle Restraints on AtSite PLAT003_ALERT_2_G Number of Uiso or Uij Restrained non-H Atoms ... PLAT042_ALERT_1_G Calc. and Reported MoietyFormula Strings Differ PLAT083_ALERT_2_G SHELXL Second Parameter in WGHT Unusually Large PLAT171_ALERT_4_G The CIF-Embedded.res File Contains EADP Records PLAT172_ALERT_4_G The CIF-Embedded .res File Contains DFIX Records PLAT175_ALERT_4_G The CIF-Embedded .res File Contains SAME Records PLAT178_ALERT_4_G The CIF-Embedded.res File Contains SIMU Records PLAT186_ALERT_4_G The CIF-Embedded .res File Contains ISOR Records PLAT187_ALERT_4_G The CIF-Embedded .res File Contains RIGU Records PLAT302_ALERT_4_G Anion/Solvent/Minor-Residue Disorder (Resd 2 ) PLAT302_ALERT_4_G Anion/Solvent/Minor-Residue Disorder (Resd 3 ) PLAT304_ALERT_4_G PLAT304_ALERT_4_G Non-Integer Number of Atoms in ..... Resd 2 PLAT411_ALERT_2_G Short Inter H...H Contact H28 ..H2SA

PLAT413_ALERT_2_G Short Inter XH3 .. XHn $1-\mathrm{x}, 1 / 2+\mathrm{y}, 3 / 2-\mathrm{z}=$

PLAT413 ALERT $2 \mathrm{G}$ short Inter XH3 .. XHn $\mathrm{H} 27, \ddot{H} 1 \mathrm{SC}$

PLAT720_ALERT_4_G Number of Unusual/Non-Standard Labels ......... PLAT789_ALERT_4_G Atoms with Negative_atom_site_disorder_group \# PLAT860_ALERT_3_G Number of Least-Squares Restraints ........... PLAT909_ALERT_3_G Percentage of I>2sig(I) Data at Theta(Max) Still PLAT910_ALERT_3_G Missing \# of FCF Reflection(s) Below Theta(Min). PLAT933_ALERT_2_G Number of OMIT Records in Embedded.res File... PLAT978_ALERT_2_G Number C-C Bonds with Positive Residual Density.

$\begin{aligned} & 12 \text { Note } \\ & 12 \text { Report } \\ & \text { Please } \text { Check } \\ & 11.70 \text { Why ? } \\ & 6 \text { Report } \\ & 1 \text { Report } \\ & 2 \text { Report } \\ & 1 \text { Report } \\ & 1 \text { Report } \\ & 1 \text { Report } \\ & 100 \% \text { Note } \\ & 100 \% \text { Note } \\ & 4.84 \text { Check } \\ & 5.16 \text { Check } \\ & 2.11 \text { Ang. } \\ & 26656 \text { Check } \\ & 1.97 \text { Ang. } \\ & 2656 \text { Check } \\ & 1.78 \text { Ang. } \\ & 466 \text { Check } \\ & 28 \text { Note } \\ & 40 \text { Check } \\ & 279 \text { Note } \\ & 30 \% \text { Note } \\ & 3 \text { Note } \\ & 6 \text { Note } \\ & 3 \text { Info }\end{aligned}$

\footnotetext{
0 ALERT level $\mathbf{A}=$ Most likely a serious problem - resolve or explain

0 ALERT level $\mathbf{B}=\mathrm{A}$ potentially serious problem, consider carefully

9 ALERT level $\mathbf{C}=$ Check. Ensure it is not caused by an omission or oversight

24 ALERT level $\mathbf{G}=$ General information/check it is not something unexpected

1 ALERT type 1 CIF construction/syntax error, inconsistent or missing data

14 ALERT type 2 Indicator that the structure model may be wrong or deficient

6 ALERT type 3 Indicator that the structure quality may be low

12 ALERT type 4 Improvement, methodology, query or suggestion

0 ALERT type 5 Informative message, check
} 
It is advisable to attempt to resolve as many as possible of the alerts in all categories. Often the minor alerts point to easily fixed oversights, errors and omissions in your CIF or refinement strategy, so attention to these fine details can be worthwhile. In order to resolve some of the more serious problems it may be necessary to carry out additional measurements or structure refinements. However, the purpose of your study may justify the reported deviations and the more serious of these should normally be commented upon in the discussion or experimental section of a paper or in the "special_details" fields of the CIF. checkCIF was carefully designed to identify outliers and unusual parameters, but every test has its limitations and alerts that are not important in a particular case may appear. Conversely, the absence of alerts does not guarantee there are no aspects of the results needing attention. It is up to the individual to critically assess their own results and, if necessary, seek expert advice.

\section{Publication of your CIF in IUCr journals}

A basic structural check has been run on your CIF. These basic checks will be run on all CIFs submitted for publication in IUCr journals (Acta Crystallographica, Journal of Applied Crystallography, Journal of Synchrotron Radiation); however, if you intend to submit to Acta Crystallographica Section C or E or IUCrData, you should make sure that full publication checks are run on the final version of your CIF prior to submission.

\section{Publication of your CIF in other journals}

Please refer to the Notes for Authors of the relevant journal for any special instructions relating to CIF submission.

\section{PLATON version of 07/08/2019; check.def file version of 30/07/2019}


Datablock 8_ArLCoBrN3R2-initial - ellipsoid plot

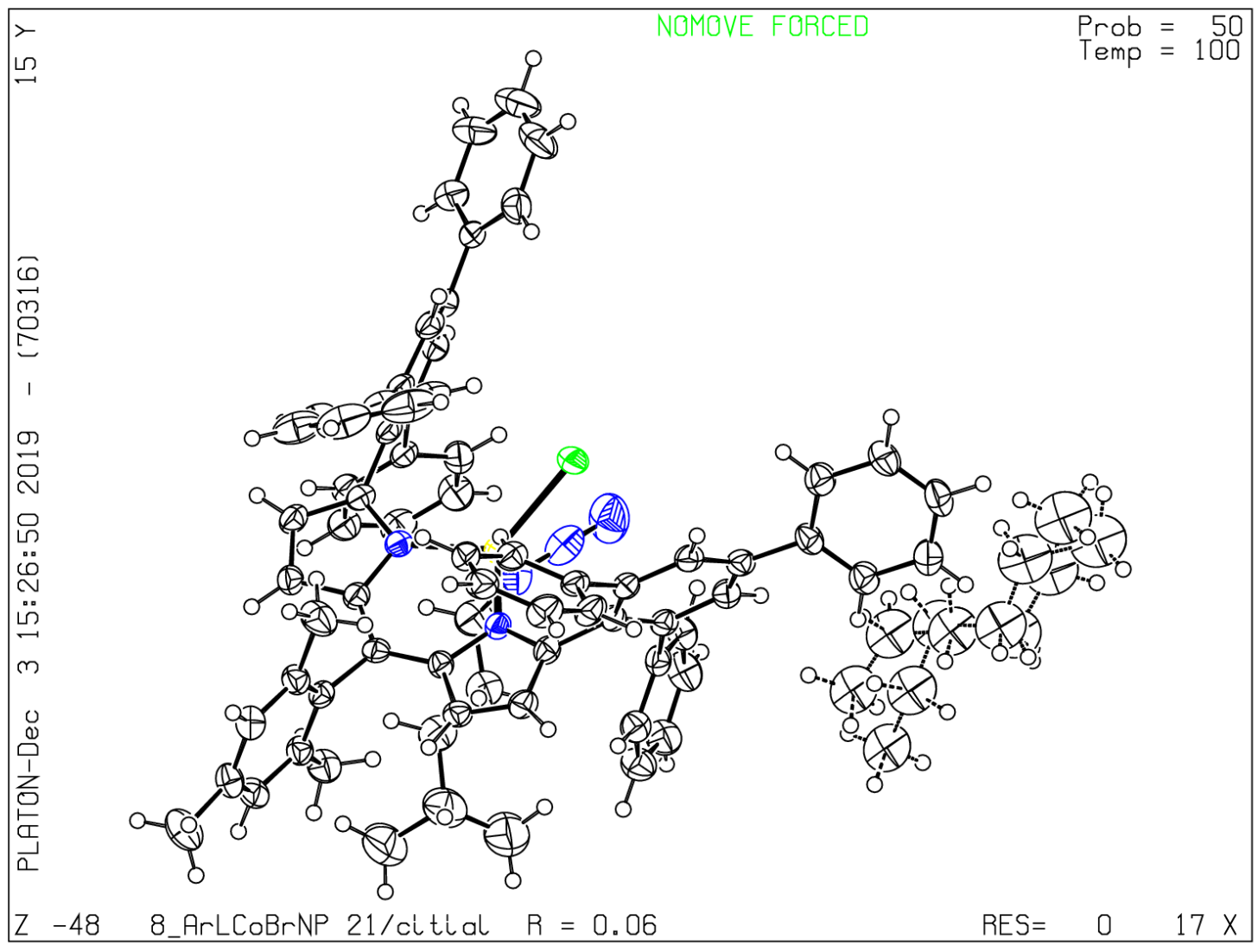




\section{checkCIF/PLATON report}

Structure factors have been supplied for datablock(s) 9_ArLCoBrNHBn

THIS REPORT IS FOR GUIDANCE ONLY. IF USED AS PART OF A REVIEW PROCEDURE FOR PUBLICATION, IT SHOULD NOT REPLACE THE EXPERTISE OF AN EXPERIENCED CRYSTALLOGRAPHIC REFEREE.

No syntax errors found. CIF dictionary Interpreting this report

\section{Datablock: 9_ArLCoBrNHBn}

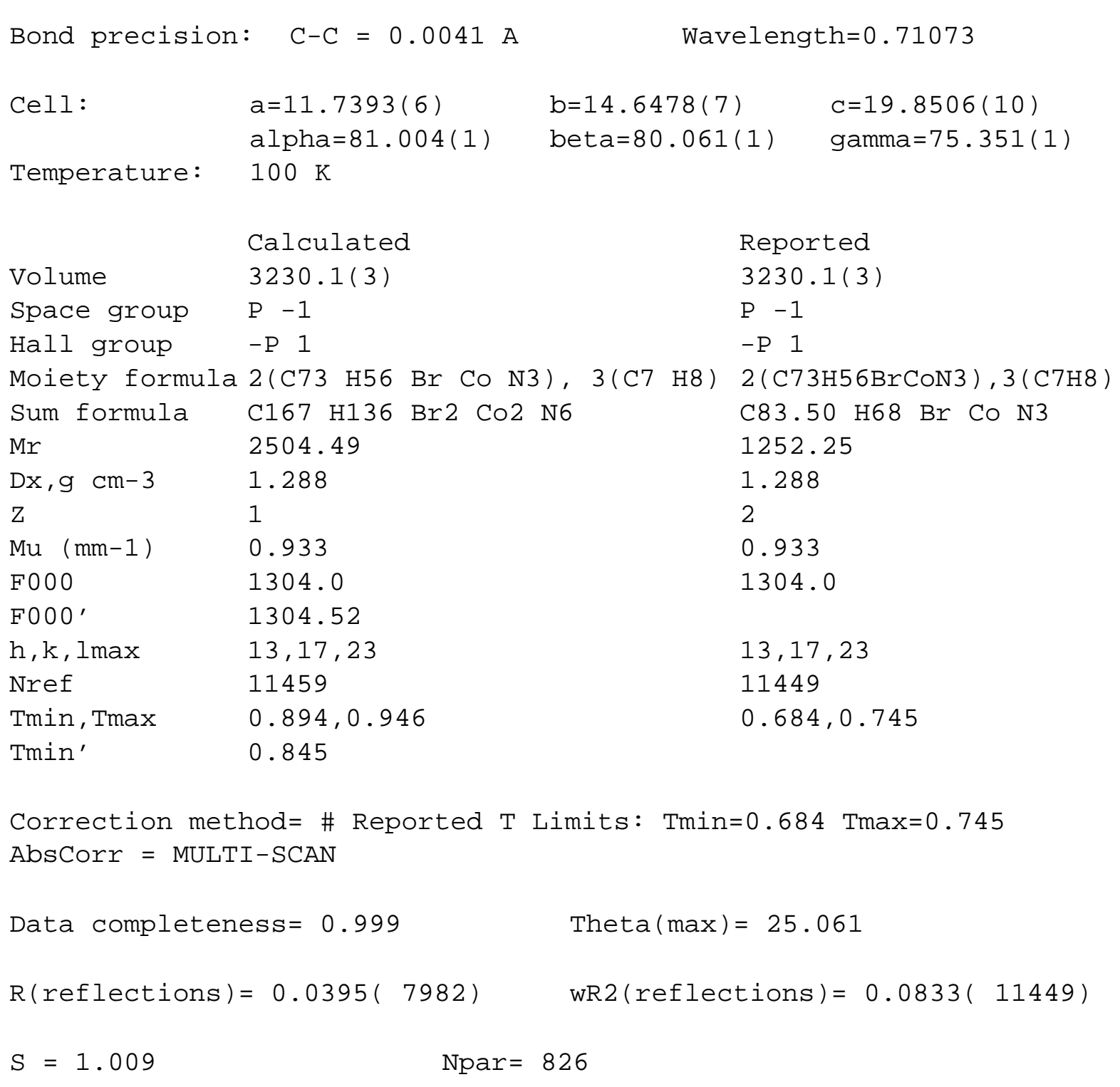

The following ALERTS were generated. Each ALERT has the format test-name_ALERT_alert-type_alert-level.

Click on the hyperlinks for more details of the test. 
Alert level $\mathrm{C}$

CRYSC01_ALERT_1_C The word below has not been recognised as a standard

identifier.

maroon

CRYSC01_ALERT_1_C No recognised colour has been given for crystal colour.

PLAT250_ALERT_2_C Large U3/U1 Ratio for Average U(i,j) Tensor....

PLAT 420_ALERT_2_C

D-H without Acceptor

N3

$--\mathrm{H} 3$

PLAT911_ALERT_3_C Missing FCF Refl Between Thmin \& STh/L= $0.596 \quad 8$ Report

0.596

Please Check

\section{Alert level G}

FORMU01_ALERT_1_G There is a discrepancy between the atom counts in the

_chemical_formula_sum and_chemical_formula_moiety. This is

usually due to the moiety formula being in the wrong format.

Atom count from_chemical_formula_sum: C83.5 H68 Br1 Co1 N3

Atom count from_chemical_formula_moiety:C167 H136 Br2 Co2 N6

PLAT003_ALERT_2_G Number of Uiso or Uij Restrained non-H Atoms ...

PLAT042_ALERT_1_G Calc. and Reported MoietyFormula Strings Differ

PLAT045_ALERT_1_G Calculated and Reported Z Differ by a Factor...

PLAT154_ALERT_1_G The s.u.'s on the Cell Angles are Equal ...(Note)

PLAT178_ALERT_4_G The CIF-Embedded .res File Contains SIMU Records

PLAT232_ALERT_2_G Hirshfeld Test Diff (M-X) Br1 .

PLAT300_ALERT_4_G Atom Site Occupancy of C1S Constrained at

PLAT300_ALERT_4_G Atom Site Occupancy of C2S

Constrained at

PLAT300_ALERT_4_G Atom Site Occupancy of C3S

Constrained at

PLAT300_ALERT_4_G Atom Site Occupancy of C4S

Constrained at

PLAT300_ALERT_4_G Atom Site Occupancy of C5S

Constrained at

PLAT300_ALERT_4_G Atom Site Occupancy of C6S

Constrained at

PLAT300_ALERT_4_G Atom Site Occupancy of C7S

PLAT300_ALERT_4_G Atom Site Occupancy of H7SA

Constrained at

Constrained at

PLAT300_ALERT_4_G Atom Site Occupancy of H1S

Constrained at

PLAT300_ALERT_4_G Atom Site Occupancy of H7SB

Constrained at

PLAT300_ALERT_4_G Atom Site Occupancy of H2S

Constrained at

Constrained at

PLAT300_ALERT_4_G Atom Site Occupancy of H7SC

Constrained at

PLAT300_ALERT_4_G Atom Site Occupancy of H3S

Constrained at

PLAT300_ALERT_4_G Atom Site Occupancy of H5S

Constrained at

PLAT302_ALERT_4_G Anion/Solvent/Minor-Residue Disorder (Resd 2 )

PLAT304_ALERT_4_G Non-Integer Number of Atoms in ..... Resd 2

PLAT720_ALERT_4_G Number of Unusual/Non-Standard Labels .........

PLAT789_ALERT_4_G Atoms with Negative_atom_site_disorder_group \#

PLAT860_ALERT_3_G Number of Least-Squares Restraints ...........

PLAT909_ALERT_3_G Percentage of I>2sig(I) Data at Theta (Max) Still

PLAT910_ALERT_3_G Missing \# of FCF Reflection(s) Below Theta(Min).

PLAT933_ALERT_2_G Number of OMIT Records in Embedded.res File...

PLAT978_ALERT_2_G Number C-C Bonds with Positive Residual Density.

7 Report

Please Check

0.50 Check

0.001 Degree

1 Report

$9.0 \mathrm{s.u}$.

0.5 Check

0.5 Check

0.5 Check

0.5 Check

0.5 Check

0.5 Check

0.5 Check

0.5 Check

0.5 Check

0.5 Check

0.5 Check

0.5 Check

0.5 Check

0.5 Check

0.5 Check

$100 \%$ Note

7.50 Check

3 Note

15 Check

42 Note

45\% Note

1 Note

1 Note

5 Info

0 ALERT level $\mathbf{A}=$ Most likely a serious problem - resolve or explain

0 ALERT level $\mathbf{B}=\mathrm{A}$ potentially serious problem, consider carefully

5 ALERT level $\mathbf{C}=$ Check. Ensure it is not caused by an omission or oversight

31 ALERT level $\mathbf{G}=$ General information/check it is not something unexpected

6 ALERT type 1 CIF construction/syntax error, inconsistent or missing data

6 ALERT type 2 Indicator that the structure model may be wrong or deficient

4 ALERT type 3 Indicator that the structure quality may be low

20 ALERT type 4 Improvement, methodology, query or suggestion

0 ALERT type 5 Informative message, check 
It is advisable to attempt to resolve as many as possible of the alerts in all categories. Often the minor alerts point to easily fixed oversights, errors and omissions in your CIF or refinement strategy, so attention to these fine details can be worthwhile. In order to resolve some of the more serious problems it may be necessary to carry out additional measurements or structure refinements. However, the purpose of your study may justify the reported deviations and the more serious of these should normally be commented upon in the discussion or experimental section of a paper or in the "special_details" fields of the CIF. checkCIF was carefully designed to identify outliers and unusual parameters, but every test has its limitations and alerts that are not important in a particular case may appear. Conversely, the absence of alerts does not guarantee there are no aspects of the results needing attention. It is up to the individual to critically assess their own results and, if necessary, seek expert advice.

\section{Publication of your CIF in IUCr journals}

A basic structural check has been run on your CIF. These basic checks will be run on all CIFs submitted for publication in IUCr journals (Acta Crystallographica, Journal of Applied Crystallography, Journal of Synchrotron Radiation); however, if you intend to submit to Acta Crystallographica Section C or E or IUCrData, you should make sure that full publication checks are run on the final version of your CIF prior to submission.

\section{Publication of your CIF in other journals}

Please refer to the Notes for Authors of the relevant journal for any special instructions relating to CIF submission.

\section{PLATON version of 07/08/2019; check.def file version of 30/07/2019}


Datablock 9_ArLCoBrNHBn - ellipsoid plot

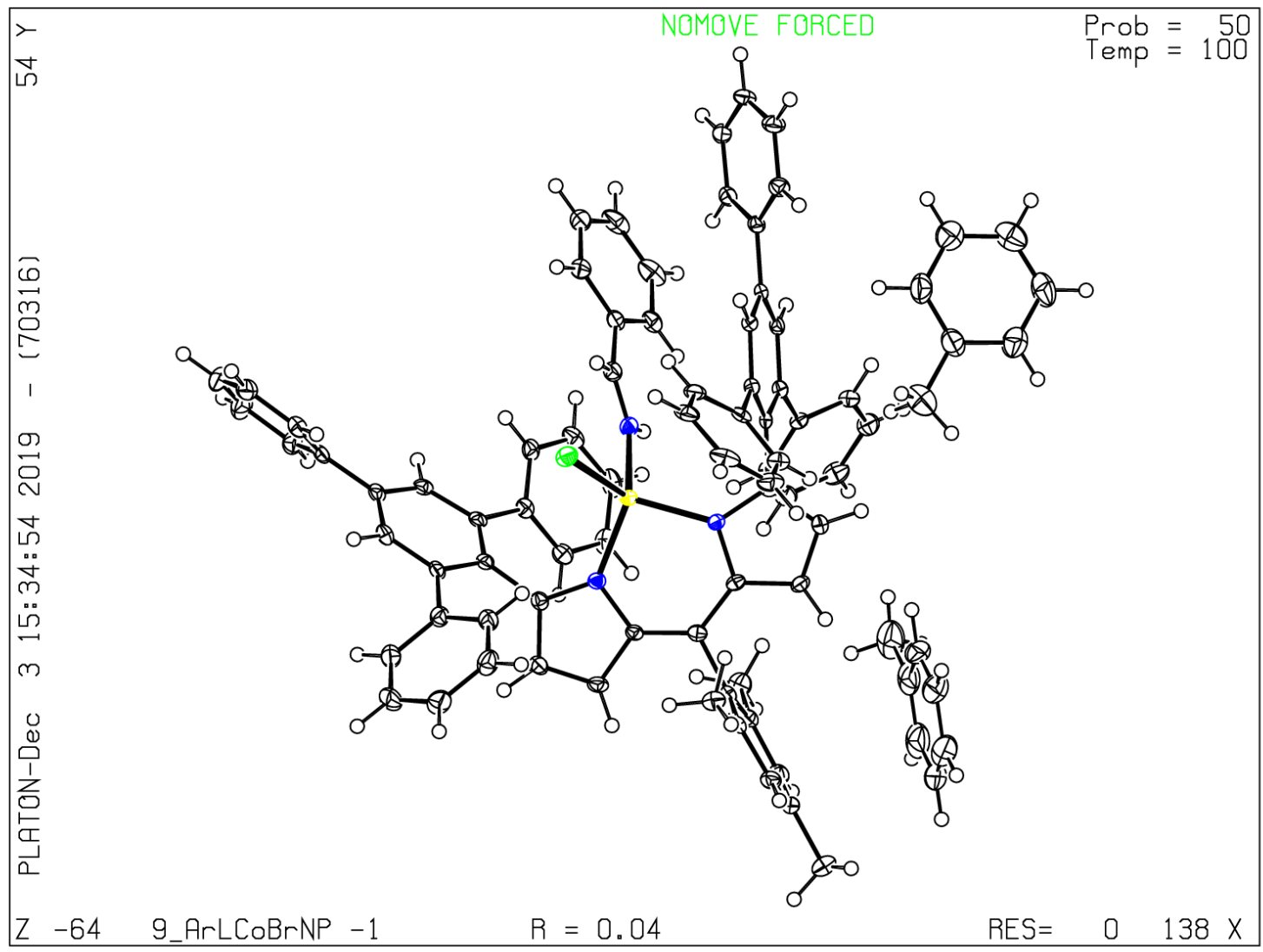




\section{checkCIF/PLATON report}

Structure factors have been supplied for datablock(s) 11_tBuLCoBr_dimer

THIS REPORT IS FOR GUIDANCE ONLY. IF USED AS PART OF A REVIEW PROCEDURE FOR PUBLICATION, IT SHOULD NOT REPLACE THE EXPERTISE OF AN EXPERIENCED CRYSTALLOGRAPHIC REFEREE.

No syntax errors found. CIF dictionary Interpreting this report

\section{Datablock: 11_tBuLCoBr_dimer}

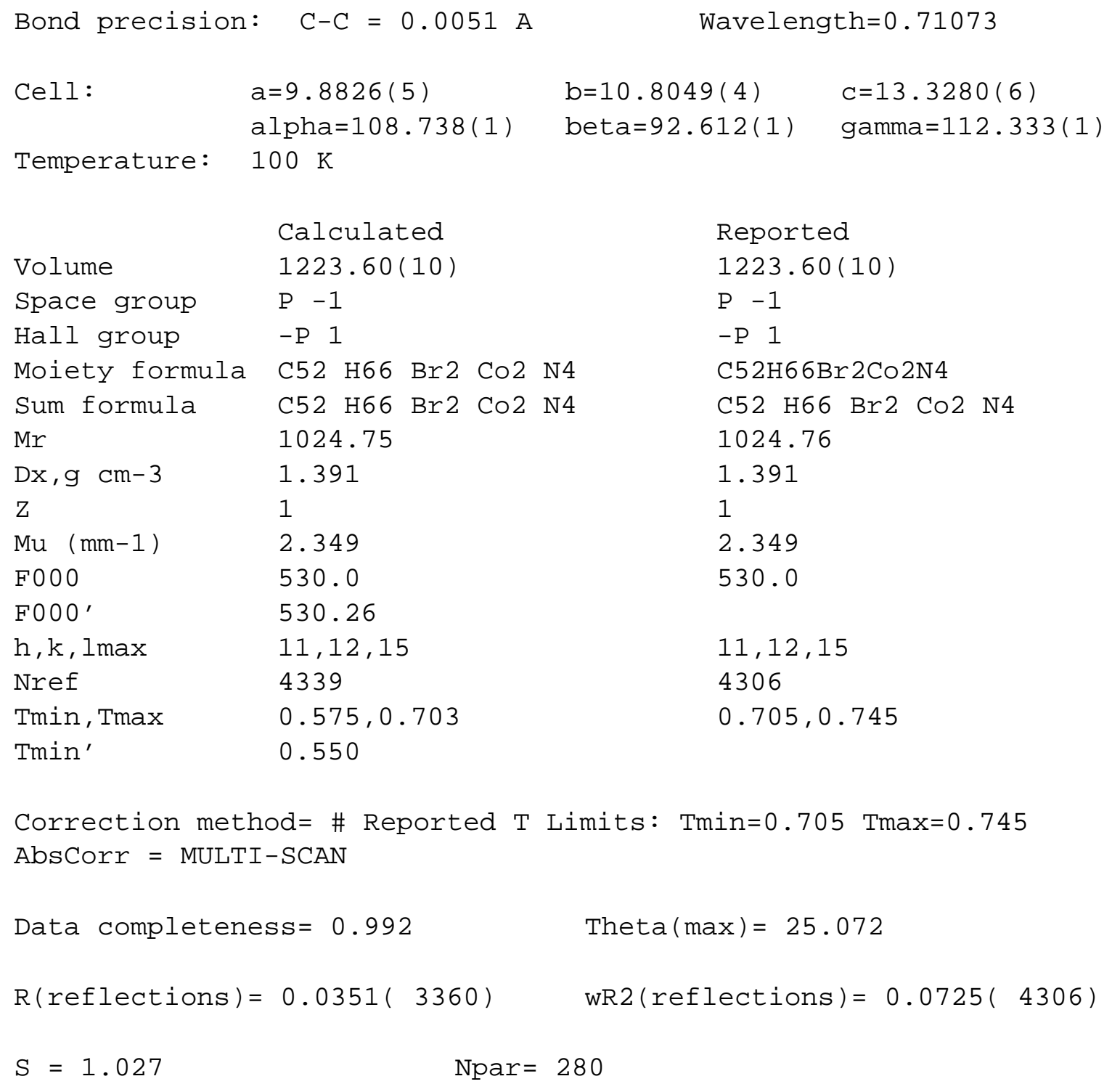


Alert level $\mathrm{C}$

PLAT911_ALERT_3_C Missing FCF Refl Between Thmin \& STh/L= $0.596 \quad 31$ Report

Alert level $\mathrm{G}$

PLAT042_ALERT_1_G Calc. and Reported MoietyFormula Strings Differ

PLAT154_ALERT_1_G The s.u.'s on the Cell Angles are Equal ..(Note)

PLAT909_ALERT_3_G Percentage of I>2sig(I) Data at Theta(Max) Still

PLAT910_ALERT_3_G Missing \# of FCF Reflection(s) Below Theta(Min). PLAT978_ALERT_2_G Number C-C Bonds with Positive Residual Density.

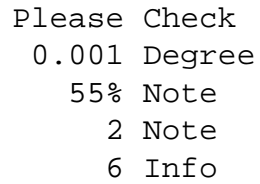

0 ALERT level $\mathbf{A}=$ Most likely a serious problem - resolve or explain

0 ALERT level $\mathbf{B}=$ A potentially serious problem, consider carefully

1 ALERT level $\mathbf{C}=$ Check. Ensure it is not caused by an omission or oversight

5 ALERT level $\mathbf{G}=$ General information/check it is not something unexpected

2 ALERT type 1 CIF construction/syntax error, inconsistent or missing data

1 ALERT type 2 Indicator that the structure model may be wrong or deficient

3 ALERT type 3 Indicator that the structure quality may be low

0 ALERT type 4 Improvement, methodology, query or suggestion

0 ALERT type 5 Informative message, check

It is advisable to attempt to resolve as many as possible of the alerts in all categories. Often the minor alerts point to easily fixed oversights, errors and omissions in your CIF or refinement strategy, so attention to these fine details can be worthwhile. In order to resolve some of the more serious problems it may be necessary to carry out additional measurements or structure refinements. However, the purpose of your study may justify the reported deviations and the more serious of these should normally be commented upon in the discussion or experimental section of a paper or in the "special_details" fields of the CIF. checkCIF was carefully designed to identify outliers and unusual parameters, but every test has its limitations and alerts that are not important in a particular case may appear. Conversely, the absence of alerts does not guarantee there are no aspects of the results needing attention. It is up to the individual to critically assess their own results and, if necessary, seek expert advice.

\section{Publication of your CIF in IUCr journals}

A basic structural check has been run on your CIF. These basic checks will be run on all CIFs submitted for publication in IUCr journals (Acta Crystallographica, Journal of Applied Crystallography, Journal of Synchrotron Radiation); however, if you intend to submit to Acta Crystallographica Section C or E or IUCrData, you should make sure that full publication checks are run on the final version of your CIF prior to submission.

\section{Publication of your CIF in other journals}

Please refer to the Notes for Authors of the relevant journal for any special instructions relating to CIF submission.

\section{PLATON version of 07/08/2019; check.def file version of 30/07/2019}


Datablock 11_tBuLCoBr_dimer - ellipsoid plot

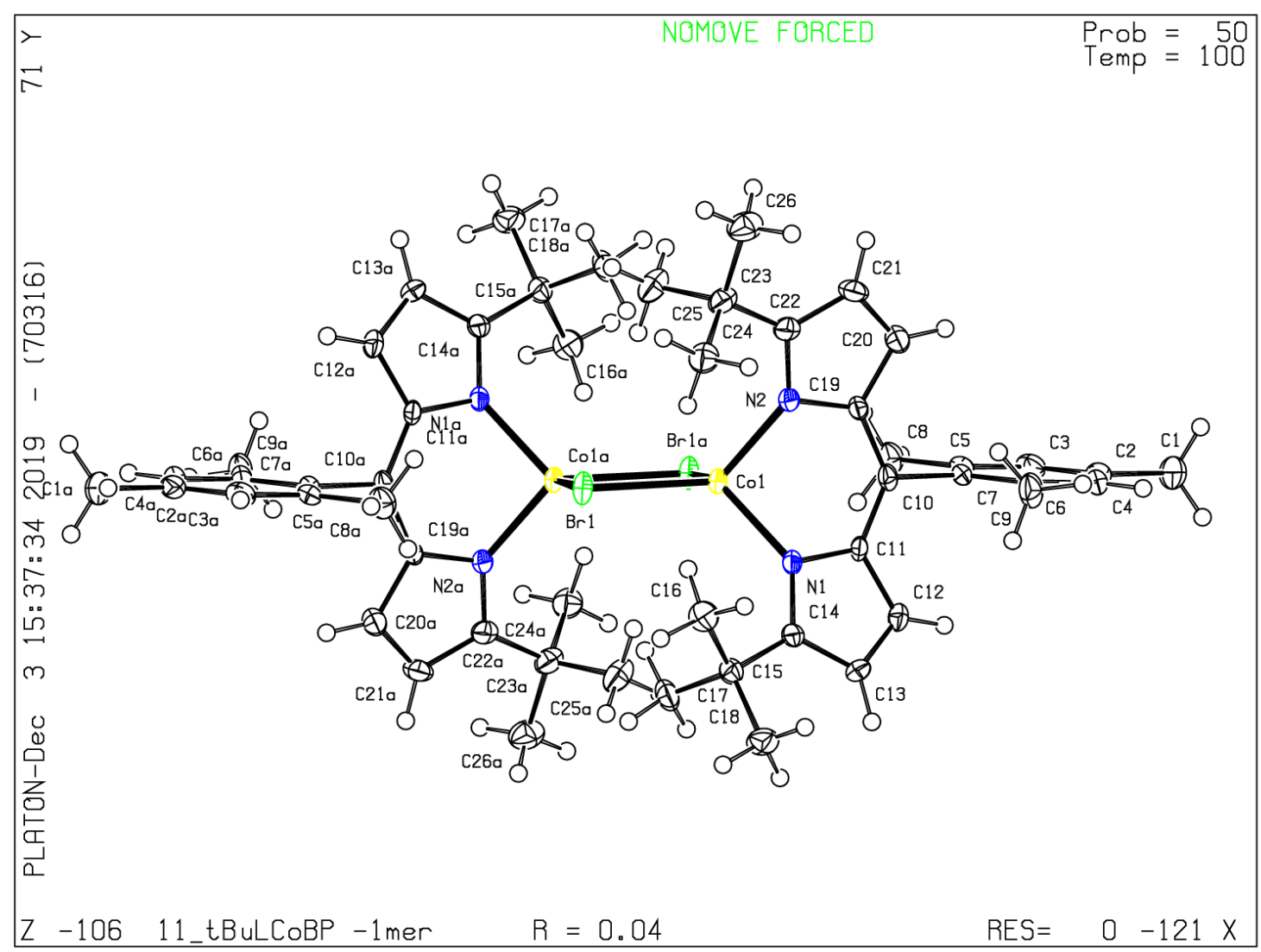




\section{checkCIF/PLATON report}

Structure factors have been supplied for datablock(s) 12_tBuLCoBrthf

THIS REPORT IS FOR GUIDANCE ONLY. IF USED AS PART OF A REVIEW PROCEDURE FOR PUBLICATION, IT SHOULD NOT REPLACE THE EXPERTISE OF AN EXPERIENCED CRYSTALLOGRAPHIC REFEREE.

No syntax errors found. CIF dictionary Interpreting this report

\section{Datablock: 12_tBuLCoBrthf}

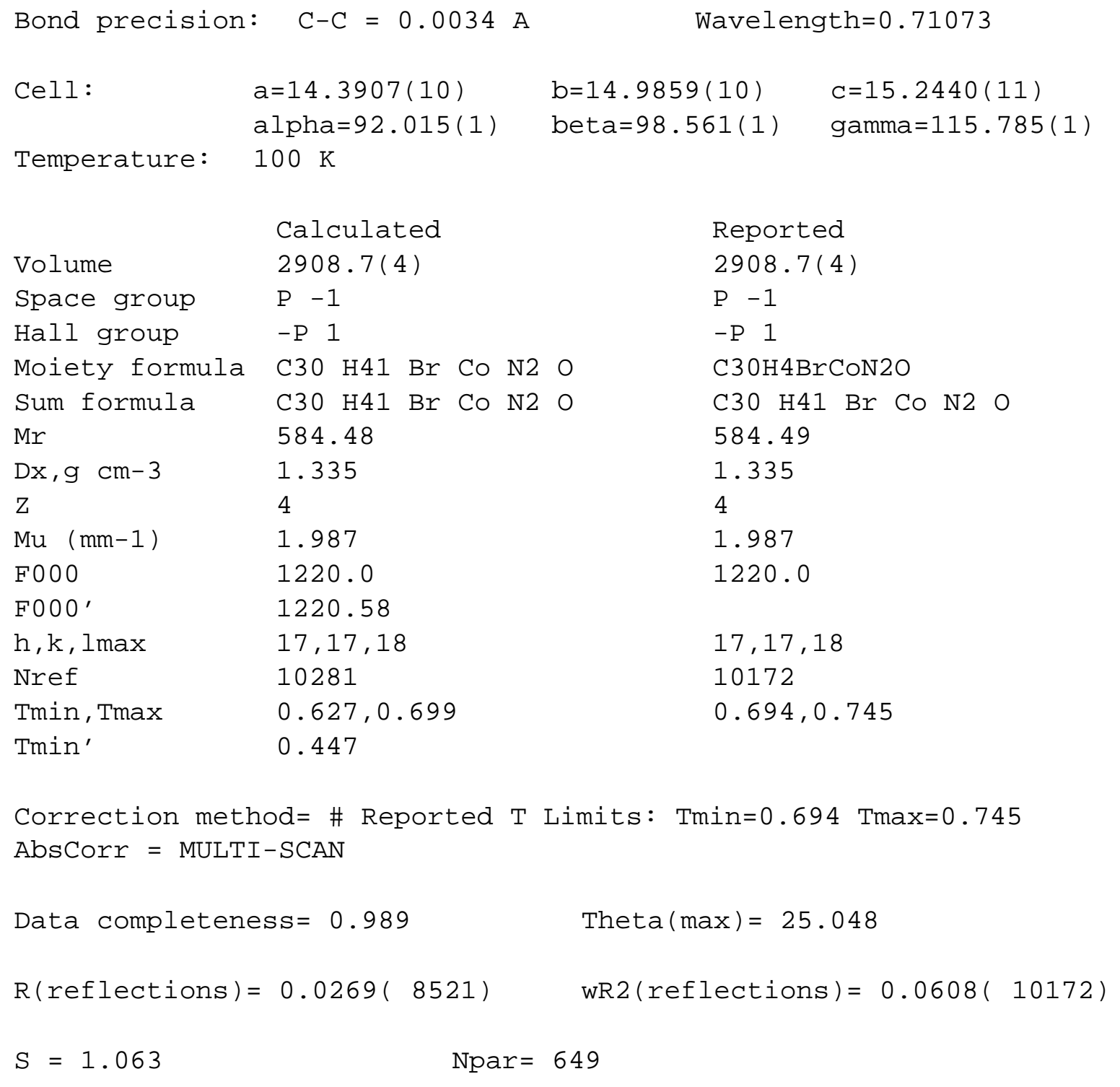


Alert level $\mathrm{C}$

PLAT601_ALERT_2_C Structure Contains Solvent Accessible VoIDS of .

PLAT911_ALERT_3_C Missing FCF Refl Between Thmin \& STh/L= 0.596

47 Ang**3

110 Report

Alert level $\mathrm{G}$

FORMU01_ALERT_1_G There is a discrepancy between the atom counts in the _chemical_formula_sum and_chemical_formula_moiety. This is usually due to the moiety formula being in the wrong format. Atom count from_chemical_formula_sum: C30 H41 Br1 Co1 N2 O1 Atom count from_chemical_formula_moiety:C30 H4 Br1 Co1 N2 O1

PLAT042_ALERT_1_G Calc. and Reported MoietyFormula Strings Differ Please Check

PLAT154_ALERT_1_G The s.u.'s on the Cell Angles are Equal ...(Note) 0.001 Degree

PLAT720_ALERT_4_G Number of Unusual/Non-Standard Labels .......... 18 Note

PLAT909_ALERT_3_G Percentage of I>2sig(I) Data at Theta(Max) Still $72 \%$ Note

PLAT978_ALERT_2_G Number C-C Bonds with Positive Residual Density. 10 Info

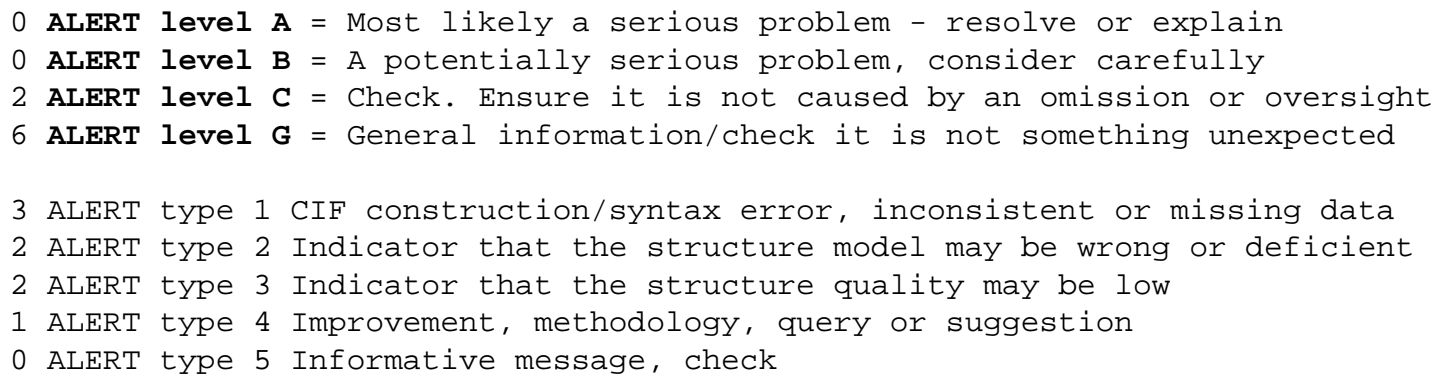

It is advisable to attempt to resolve as many as possible of the alerts in all categories. Often the minor alerts point to easily fixed oversights, errors and omissions in your CIF or refinement strategy, so attention to these fine details can be worthwhile. In order to resolve some of the more serious problems it may be necessary to carry out additional measurements or structure refinements. However, the purpose of your study may justify the reported deviations and the more serious of these should normally be commented upon in the discussion or experimental section of a paper or in the "special_details" fields of the CIF. checkCIF was carefully designed to identify outliers and unusual parameters, but every test has its limitations and alerts that are not important in a particular case may appear. Conversely, the absence of alerts does not guarantee there are no aspects of the results needing attention. It is up to the individual to critically assess their own results and, if necessary, seek expert advice.

\section{Publication of your CIF in IUCr journals}

A basic structural check has been run on your CIF. These basic checks will be run on all CIFs submitted for publication in IUCr journals (Acta Crystallographica, Journal of Applied Crystallography, Journal of Synchrotron Radiation); however, if you intend to submit to Acta Crystallographica Section C or E or IUCrData, you should make sure that full publication checks are run on the final version of your CIF prior to submission.

\section{Publication of your CIF in other journals}

Please refer to the Notes for Authors of the relevant journal for any special instructions relating to CIF submission. 
PLATON version of 07/08/2019; check.def file version of 30/07/2019

Datablock 12_tBuLCoBrthf - ellipsoid plot

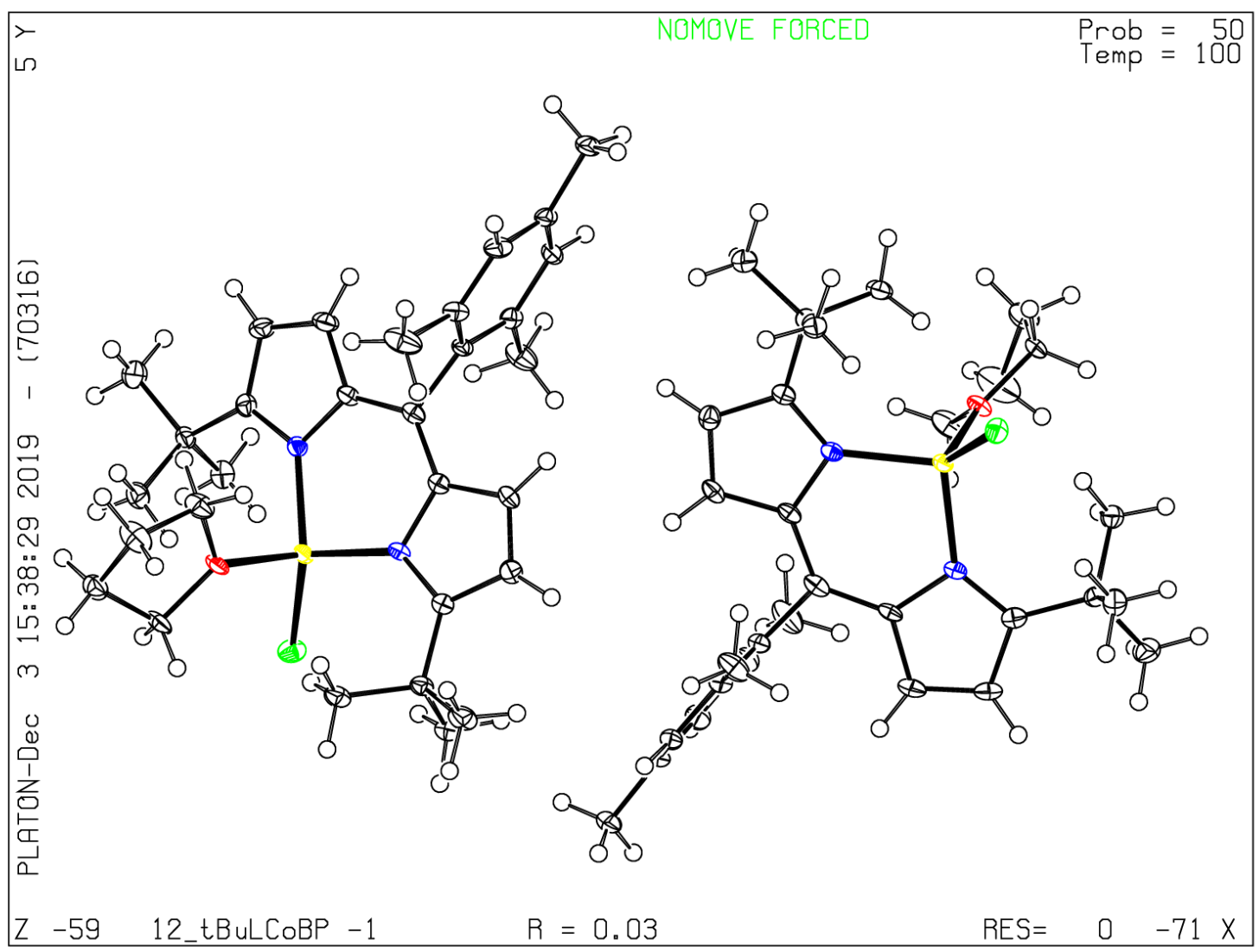




\section{checkCIF/PLATON report}

Structure factors have been supplied for datablock(s) 14_tBuLCoBrtriazole

THIS REPORT IS FOR GUIDANCE ONLY. IF USED AS PART OF A REVIEW PROCEDURE FOR PUBLICATION, IT SHOULD NOT REPLACE THE EXPERTISE OF AN EXPERIENCED CRYSTALLOGRAPHIC REFEREE.

No syntax errors found. CIF dictionary Interpreting this report

\section{Datablock: 14_tBuLCoBrtriazole}

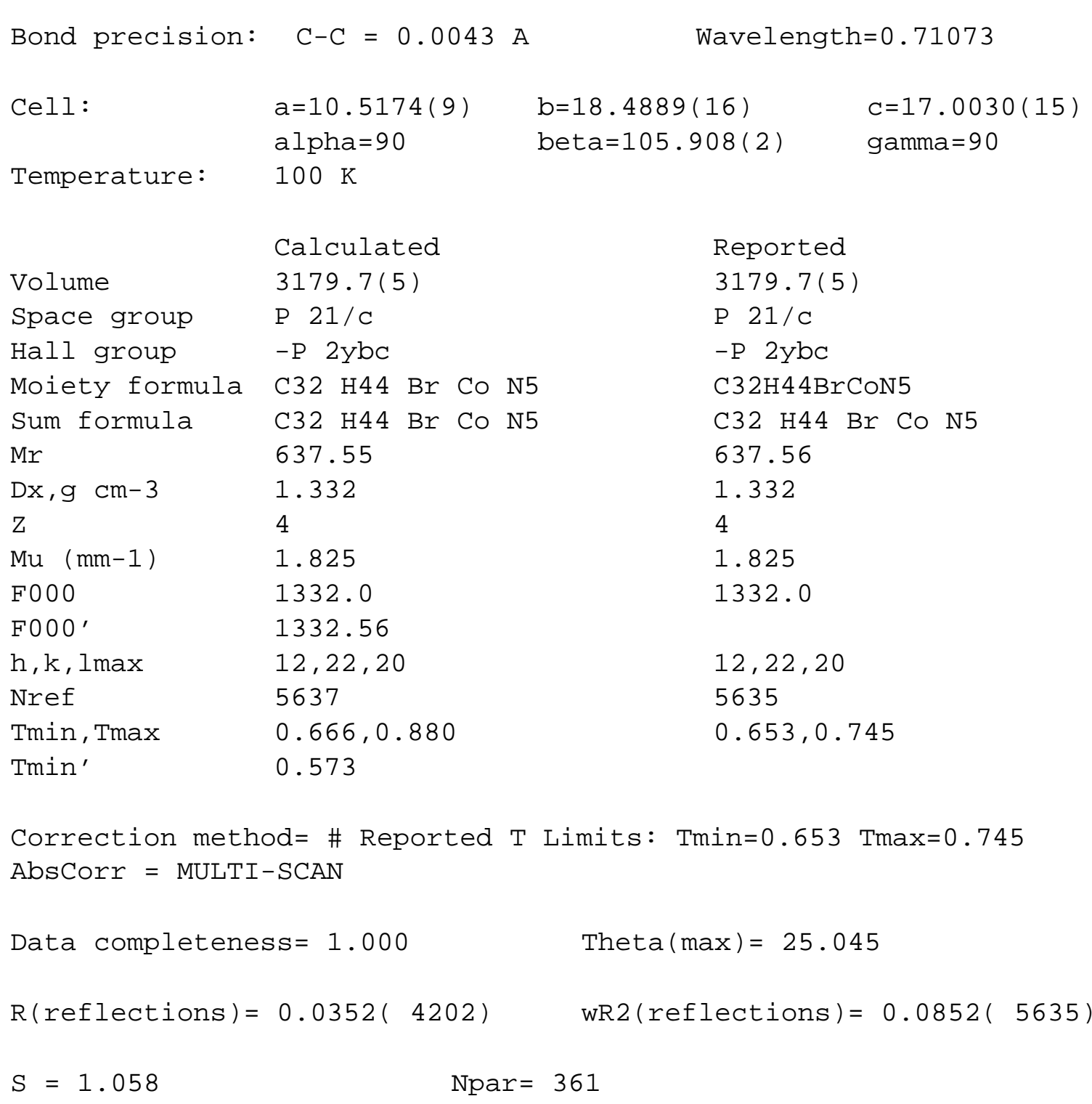

The following ALERTS were generated. Each ALERT has the format test-name_ALERT_alert-type_alert-level.

Click on the hyperlinks for more details of the test. 
Alert level C

PLAT094_ALERT_2_C Ratio of Maximum / Minimum Residual Density ....

PLAT220_ALERT_2_C Non-Solvent Resd 1 C Ueq (max)/Ueq(min) Range

PLAT911_ALERT_3_C Missing FCF Refl Between Thmin \& STh/L $=0.596$

2.38 Report

3.6 Ratio

4 Report

Alert level $\mathbf{G}$

PLAT042_ALERT_1_G Calc. and Reported MoietyFormula Strings Differ

PLAT793_ALERT_4_G Model has Chirality at C28 (Centro SPGR)

PLAT909_ALERT_3_G Percentage of I>2sig(I) Data at Theta(Max) Still

PLAT978_ALERT_2_G Number C-C Bonds with Positive Residual Density.
Please Check
R Verify
$52 \%$ Note
3 Info

It is advisable to attempt to resolve as many as possible of the alerts in all categories. Often the minor alerts point to easily fixed oversights, errors and omissions in your CIF or refinement strategy, so attention to these fine details can be worthwhile. In order to resolve some of the more serious problems it may be necessary to carry out additional measurements or structure refinements. However, the purpose of your study may justify the reported deviations and the more serious of these should normally be commented upon in the discussion or experimental section of a paper or in the "special_details" fields of the CIF. checkCIF was carefully designed to identify outliers and unusual parameters, but every test has its limitations and alerts that are not important in a particular case may appear. Conversely, the absence of alerts does not guarantee there are no aspects of the results needing attention. It is up to the individual to critically assess their own results and, if necessary, seek expert advice.

\section{Publication of your CIF in IUCr journals}

A basic structural check has been run on your CIF. These basic checks will be run on all CIFs submitted for publication in IUCr journals (Acta Crystallographica, Journal of Applied Crystallography, Journal of Synchrotron Radiation); however, if you intend to submit to Acta Crystallographica Section $C$ or $E$ or IUCrData, you should make sure that full publication checks are run on the final version of your CIF prior to submission.

\section{Publication of your CIF in other journals}

Please refer to the Notes for Authors of the relevant journal for any special instructions relating to CIF submission.

\section{PLATON version of 07/08/2019; check.def file version of 30/07/2019}


Datablock 14_tBuLCoBrtriazole - ellipsoid plot

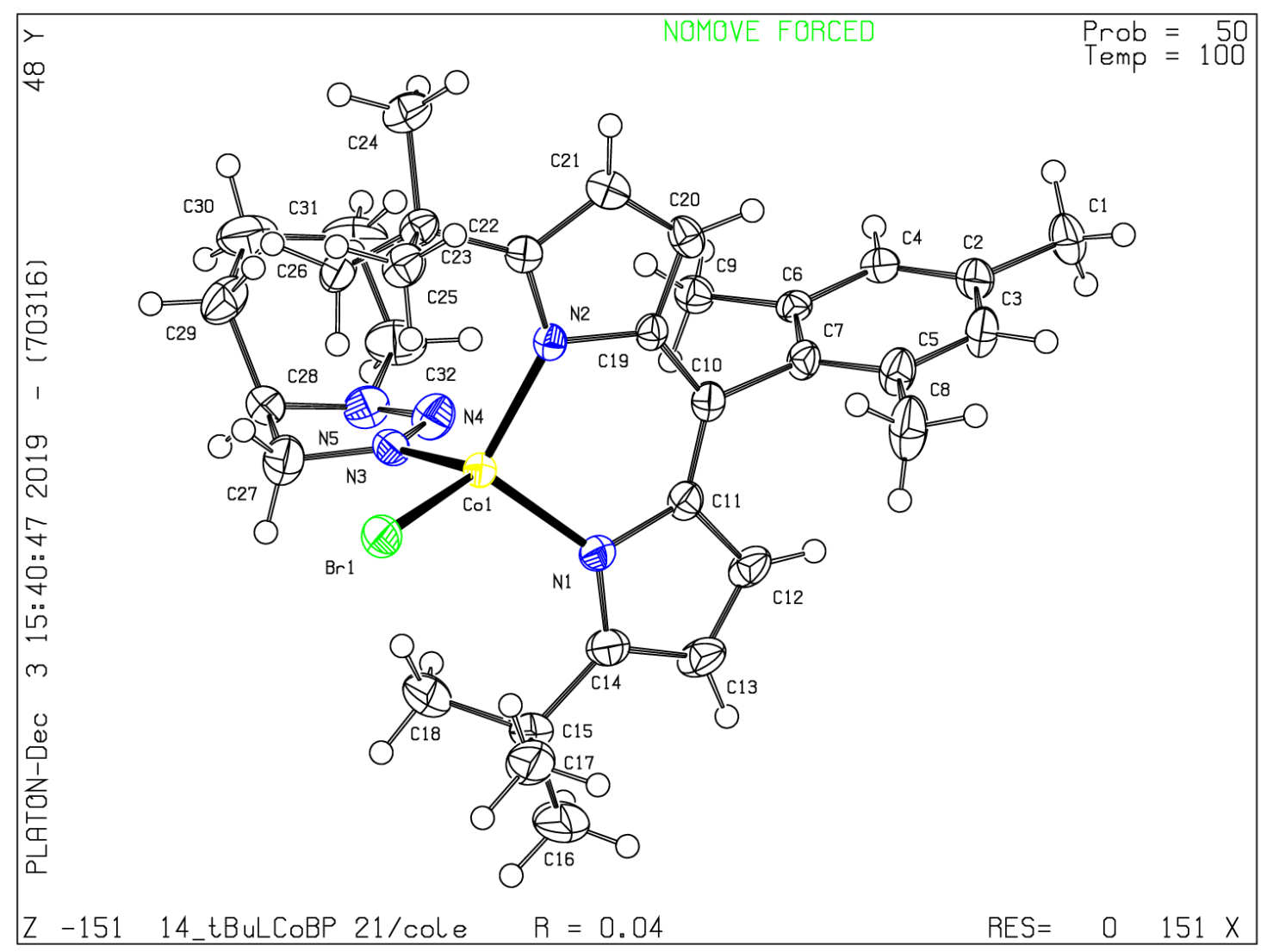




\section{checkCIF/PLATON report}

Structure factors have been supplied for datablock(s) 16_tBuLCoBr2-phenylpyrrolidine

THIS REPORT IS FOR GUIDANCE ONLY. IF USED AS PART OF A REVIEW PROCEDURE FOR PUBLICATION, IT SHOULD NOT REPLACE THE EXPERTISE OF AN EXPERIENCED CRYSTALLOGRAPHIC REFEREE.

No syntax errors found. CIF dictionary Interpreting this report

\section{Datablock: 16_tBuLCoBr2-phenylpyrrolidine}

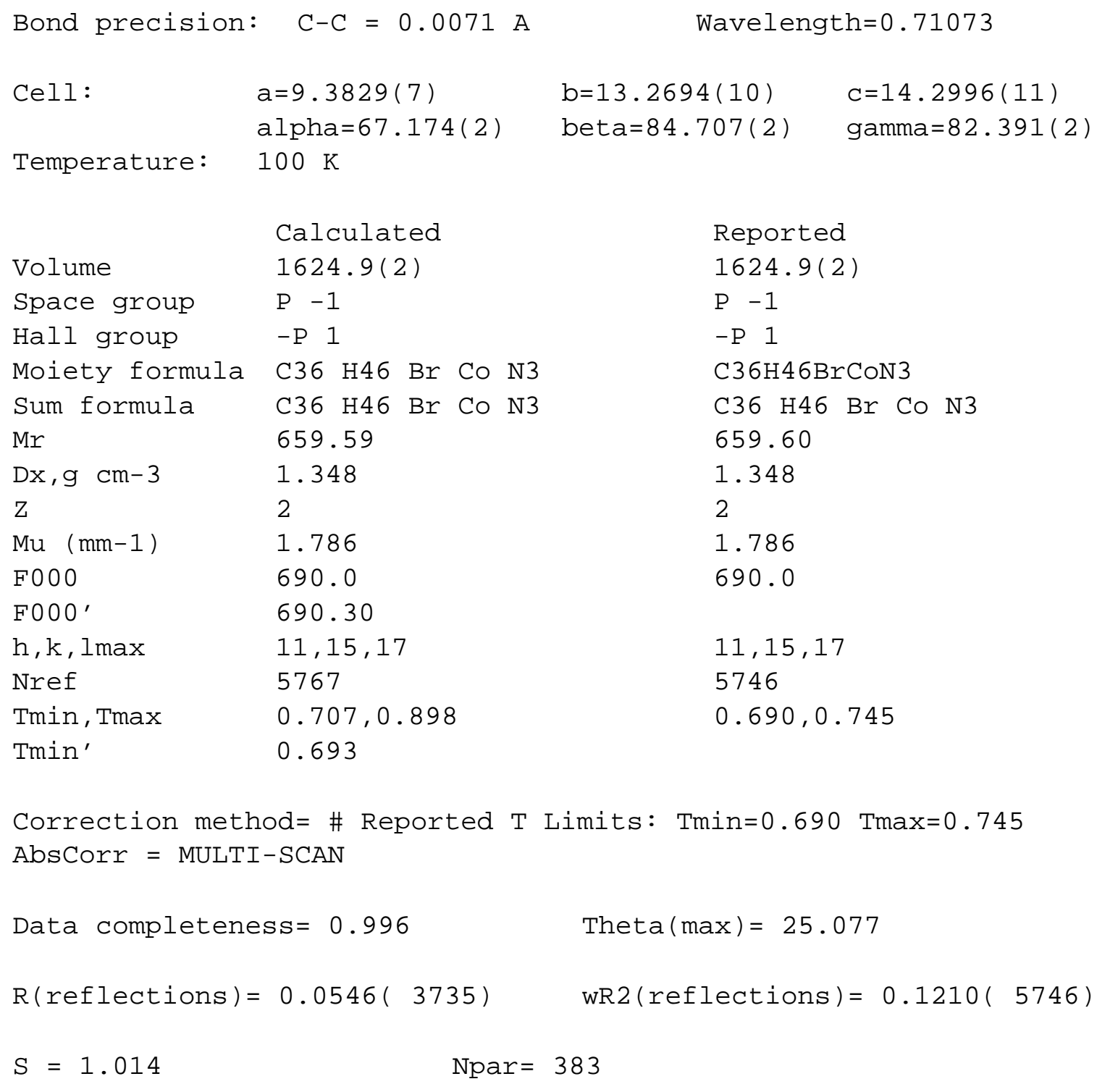


Alert level $\mathrm{C}$

PLAT094_ALERT_2_C Ratio of Maximum / Minimum Residual Density ....

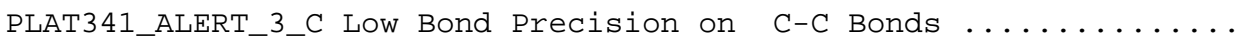

PLAT906_ALERT_3_C Large $K$ Value in the Analysis of Variance ......

PLAT910_ALERT_3_C Missing \# of FCF Reflection(s) Below Theta(Min).

PLAT911_ALERT_3_C Missing FCF Refl Between Thmin \& STh/L= 0.596

PLAT978_ALERT_2_C Number C-C Bonds with Positive Residual Density.
2.40 Report
0.00708 Ang.
2.954 Check
5 Note
16 Report
0 Info

Alert level G

PLAT042_ALERT_1_G Calc. and Reported MoietyFormula Strings Differ

PLAT154_ALERT_1_G The s.u.'s on the Cell Angles are Equal .. (Note)

PLAT793_ALERT_4_G Model has Chirality at N3 (Centro SPGR)

PLAT793_ALERT_4_G Model has Chirality at C30 (Centro SPGR)

PLAT909_ALERT_3_G Percentage of I>2sig(I) Data at Theta(Max) Still
Please Check
0.002 Degree
S Verify
R Verify
$32 \%$ Note

It is advisable to attempt to resolve as many as possible of the alerts in all categories. Often the minor alerts point to easily fixed oversights, errors and omissions in your CIF or refinement strategy, so attention to these fine details can be worthwhile. In order to resolve some of the more serious problems it may be necessary to carry out additional measurements or structure refinements. However, the purpose of your study may justify the reported deviations and the more serious of these should normally be commented upon in the discussion or experimental section of a paper or in the "special_details" fields of the CIF. checkCIF was carefully designed to identify outliers and unusual parameters, but every test has its limitations and alerts that are not important in a particular case may appear. Conversely, the absence of alerts does not guarantee there are no aspects of the results needing attention. It is up to the individual to critically assess their own results and, if necessary, seek expert advice.

\section{Publication of your CIF in IUCr journals}

A basic structural check has been run on your CIF. These basic checks will be run on all CIFs submitted for publication in IUCr journals (Acta Crystallographica, Journal of Applied Crystallography, Journal of Synchrotron Radiation); however, if you intend to submit to Acta Crystallographica Section C or E or IUCrData, you should make sure that full publication checks are run on the final version of your CIF prior to submission.

\section{Publication of your CIF in other journals}

Please refer to the Notes for Authors of the relevant journal for any special instructions relating to CIF submission. 


\section{PLATON version of 07/08/2019; check.def file version of 30/07/2019}

Datablock 16_tBuLCoBr2-phenylpyrrolidine - ellipsoid plot

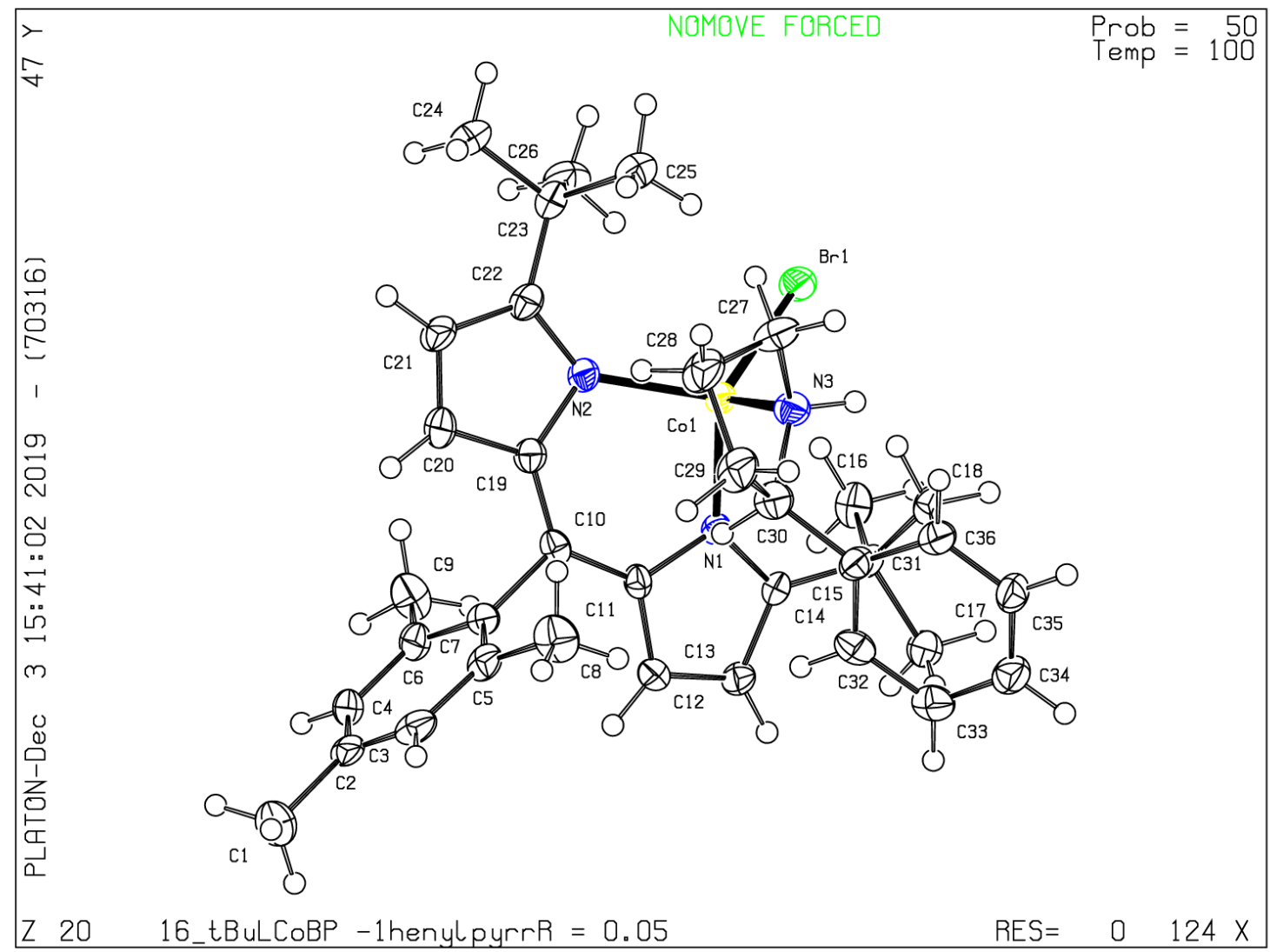




\section{checkCIF/PLATON report}

Structure factors have been supplied for datablock(s) 18_tBuLCoCl22-dimethylpyrrolidine

THIS REPORT IS FOR GUIDANCE ONLY. IF USED AS PART OF A REVIEW PROCEDURE FOR PUBLICATION, IT SHOULD NOT REPLACE THE EXPERTISE OF AN EXPERIENCED CRYSTALLOGRAPHIC REFEREE.

No syntax errors found. CIF dictionary Interpreting this report

\section{Datablock: 18_tBuLCoCl22-dimethylpyrrolidine}

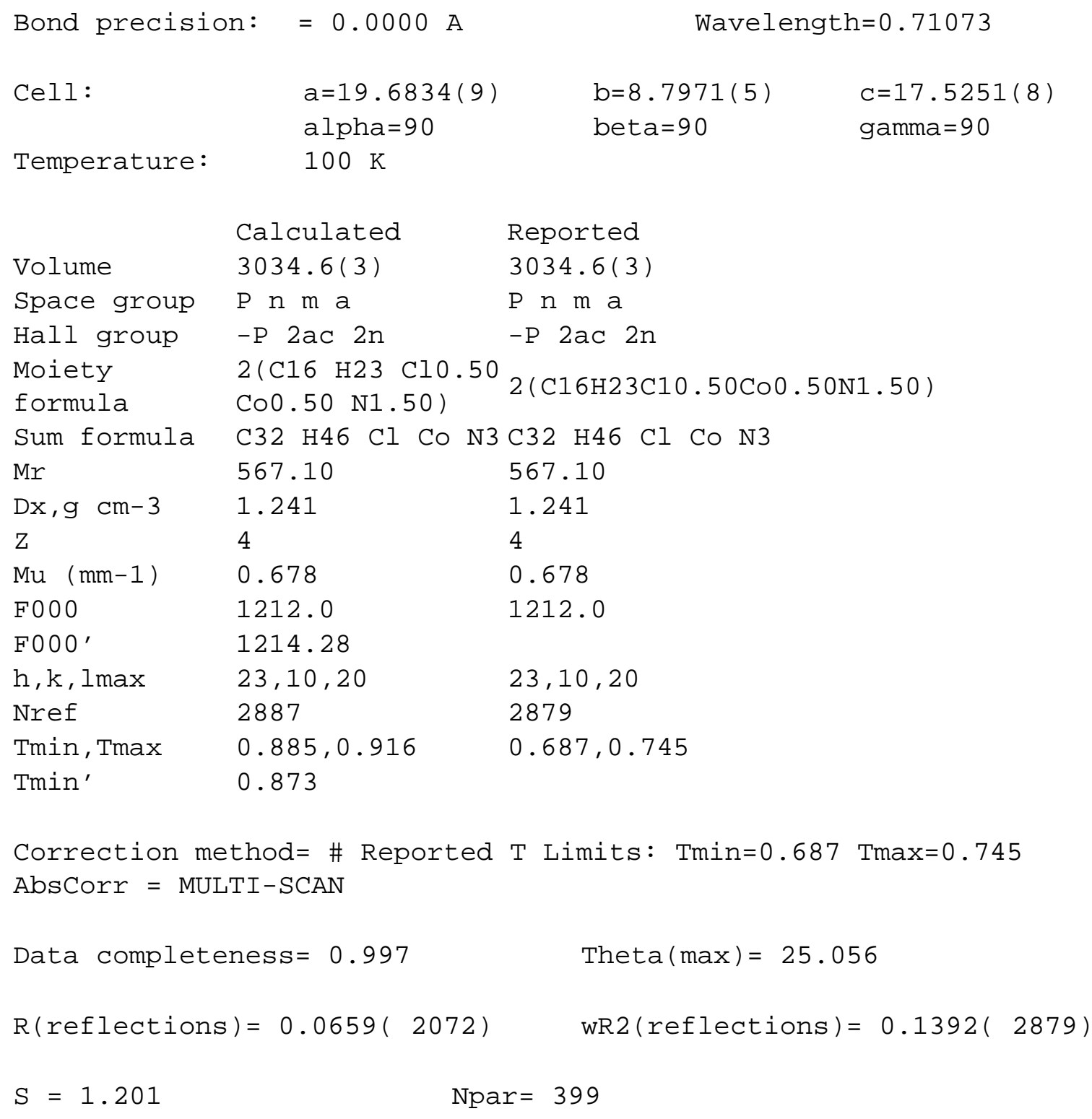


The following ALERTS were generated. Each ALERT has the format

test-name_ALERT_alert-type_alert-level.

Click on the hyperlinks for more details of the test.

Alert level B

PLAT088_ALERT_3_B Poor Data / Parameter Ratio ................. 7.22 Note

Author Response: This structure is in a high symmetry space group (Pnma). It is

full-molecule disorder along a mirror plane, and the 2,2-dimethylpyrrolidine moiety is further disorder in two positions. The restraints on bond lengths and atomic displacement parameters have been applied to improve the observed Data/Parameter ratio and stablize the model.

Alert level C
\begin{tabular}{|l|l|l|l} 
PLAT215_ALERT_3_C Disordered C10 & has ADP max/min Ratio ..... & 3.2 Note \\
PLAT220_ALERT_2_C Non-Solvent Resd 1 C Ueq (max)/Ueq(min) Range & 3.7 Ratio \\
PLAT906_ALERT_3_C Large K Value in the Analysis of Variance ...... & 8.871 Check \\
PLAT906_ALERT_3_C Large K Value in the Analysis of Variance ..... & 2.576 Check \\
PLAT911_ALERT_3_C Missing FCF Refl Between Thmin \& STh/L= 0.596 & 6 Report
\end{tabular}

Alert level $\mathbf{G}$

FORMU01_ALERT_1_G There is a discrepancy between the atom counts in the

_chemical_formula_sum and_chemical_formula_moiety. This is

usually due to the moiety formula being in the wrong format.

Atom count from_chemical_formula_sum: C32 H46 Cl1 Co1 N3

Atom count from_chemical_formula_moiety:C53 H46 Co1 N3

PLAT002_ALERT_2_G Number of Distance or Angle Restraints on AtSite

PLAT003_ALERT_2_G Number of Uiso or Uij Restrained non-H Atoms ...

PLAT007_ALERT_5_G Number of Unrefined Donor-H Atoms ...........

PLAT042_ALERT_1_G Calc. and Reported MoietyFormula Strings Differ

PLAT083_ALERT_2_G SHELXL Second Parameter in WGHT Unusually Large

PLAT174_ALERT_4_G The CIF-Embedded .res File Contains FLAT Records

PLAT175_ALERT_4_G The CIF-Embedded.res File Contains SAME Records

PLAT176_ALERT_4_G The CIF-Embedded.res File Contains SADI Records

PLAT178_ALERT_4_G The CIF-Embedded .res File Contains SIMU Records

PLAT187_ALERT_4_G The CIF-Embedded.res File Contains RIGU Records

PLAT300_ALERT_4_G Atom Site Occupancy of Co1 Constrained at

PLAT300_ALERT_4_G Atom Site Occupancy of Cl1 Constrained at

PLAT300_ALERT_4_G Atom Site Occupancy of N1

Constrained at

PLAT300_ALERT_4_G Atom Site Occupancy of N2

PLAT300_ALERT_4_G Atom Site Occupancy of C1

PLAT300_ALERT_4_G Atom Site Occupancy of C2

Constrained at

Constrained at

Constrained at

PLAT300_ALERT_4_G Atom Site Occupancy of C3

Constrained at

PLAT300_ALERT_4_G Atom Site Occupancy of C4

PLAT300_ALERT_4_G Atom Site Occupancy of C5

Constrained at

Constrained at

PLAT300_ALERT_4_G Atom Site Occupancy of C6

PLAT300_ALERT_4_G Atom Site Occupancy of C7

PLAT300_ALERT_4_G Atom Site Occupancy of C8

PLAT300_ALERT_4_G Atom Site Occupancy of C9

PLAT300_ALERT_4_G Atom Site Occupancy of C10

PLAT300_ALERT_4_G Atom Site Occupancy of C11

PLAT300_ALERT_4_G Atom Site Occupancy of C12

PLAT300_ALERT_4_G Atom Site Occupancy of C13

PLAT300_ALERT_4_G Atom Site Occupancy of C14

Constrained at

Constrained at

Constrained at

Constrained at

Constrained at

Constrained at

Constrained at

Constrained at

Constrained at

PLAT300_ALERT_4_G Atom Site Occupancy of C15

Constrained at

$\begin{aligned} 23 & \text { Note } \\ 42 & \text { Report } \\ 2 & \text { Report } \\ \text { Please } & \text { Check } \\ 8.34 & \text { Why ? } \\ 1 & \text { Report } \\ 3 & \text { Report } \\ 3 & \text { Report } \\ 1 & \text { Report } \\ 1 & \text { Report } \\ 0.5 & \text { Check } \\ 0.5 & \text { Check } \\ 0.5 & \text { Check } \\ 0.5 & \text { Check } \\ 0.5 & \text { Check } \\ 0.5 & \text { Check } \\ 0.5 & \text { Check } \\ 0.5 & \text { Check } \\ 0.5 & \text { Check } \\ 0.5 & \text { Check } \\ 0.5 & \text { Check } \\ 0.5 & \text { Check } \\ 0.5 & \text { Check } \\ 0.5 & \text { Check } \\ 0.5 & \text { Check } \\ 0.5 & \text { Check } \\ 0.5 & \text { Check } \\ 0.5 & \text { Check } \\ 0.5 & \text { Check }\end{aligned}$




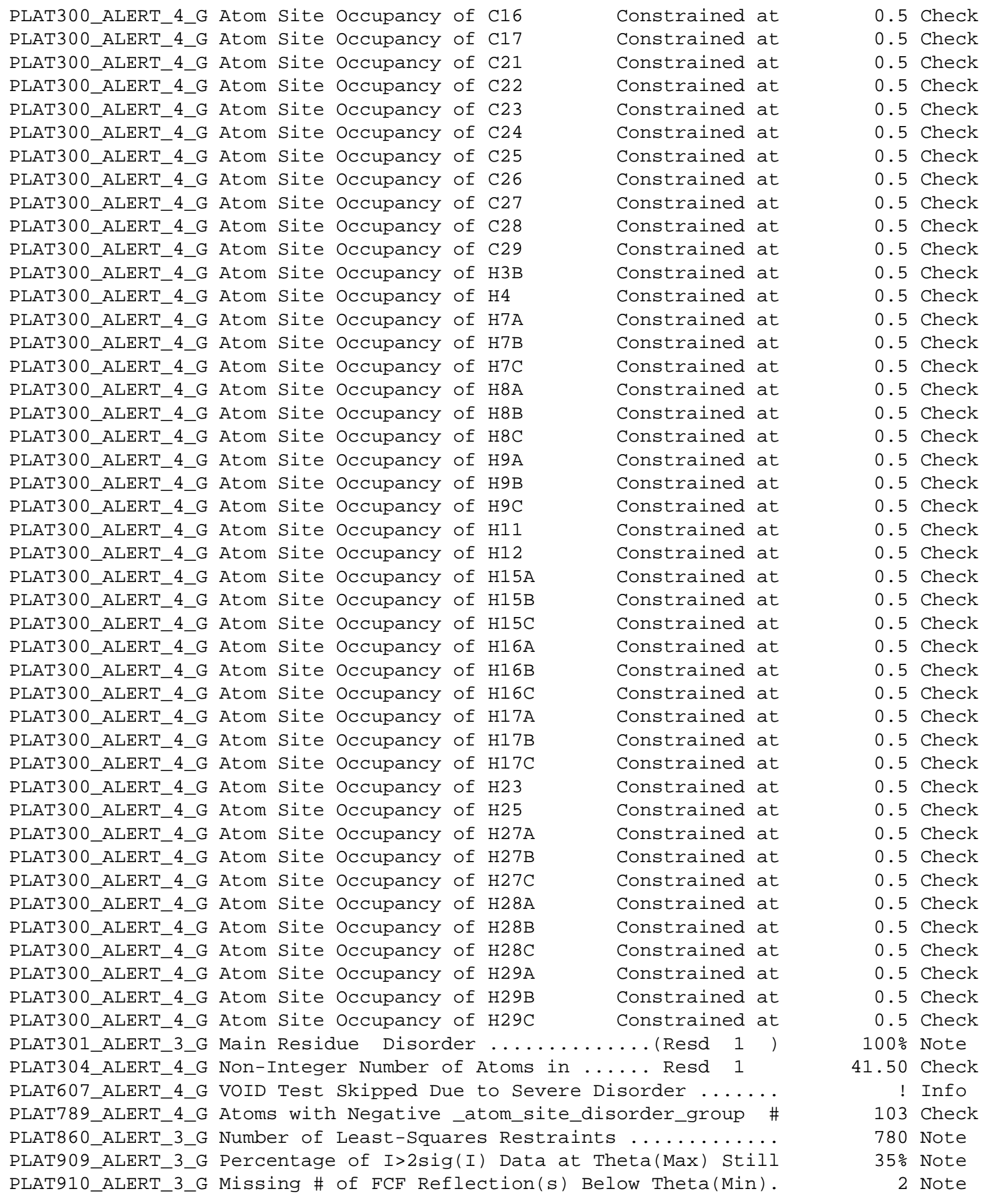

0 ALERT level $\mathbf{A}=$ Most likely a serious problem - resolve or explain

1 ALERT level $\mathbf{B}=\mathrm{A}$ potentially serious problem, consider carefully

5 ALERT level $\mathbf{C}=$ Check. Ensure it is not caused by an omission or oversight

81 ALERT level $\mathbf{G}=$ General information/check it is not something unexpected

2 ALERT type 1 CIF construction/syntax error, inconsistent or missing data 
4 ALERT type 2 Indicator that the structure model may be wrong or deficient

9 ALERT type 3 Indicator that the structure quality may be low

71 ALERT type 4 Improvement, methodology, query or suggestion

1 ALERT type 5 Informative message, check

It is advisable to attempt to resolve as many as possible of the alerts in all categories. Often the minor alerts point to easily fixed oversights, errors and omissions in your CIF or refinement strategy, so attention to these fine details can be worthwhile. In order to resolve some of the more serious problems it may be necessary to carry out additional measurements or structure refinements. However, the purpose of your study may justify the reported deviations and the more serious of these should normally be commented upon in the discussion or experimental section of a paper or in the "special_details" fields of the CIF. checkCIF was carefully designed to identify outliers and unusual parameters, but every test has its limitations and alerts that are not important in a particular case may appear. Conversely, the absence of alerts does not guarantee there are no aspects of the results needing attention. It is up to the individual to critically assess their own results and, if necessary, seek expert advice.

\section{Publication of your CIF in IUCr journals}

A basic structural check has been run on your CIF. These basic checks will be run on all CIFs submitted for publication in IUCr journals (Acta Crystallographica, Journal of Applied Crystallography, Journal of Synchrotron Radiation); however, if you intend to submit to Acta Crystallographica Section C or E or IUCrData, you should make sure that full publication checks are run on the final version of your CIF prior to submission.

\section{Publication of your CIF in other journals}

Please refer to the Notes for Authors of the relevant journal for any special instructions relating to CIF submission.

\section{PLATON version of 07/08/2019; check.def file version of 30/07/2019}


Datablock 18_tBuLCoCl22-dimethylpyrrolidine - ellipsoid plot

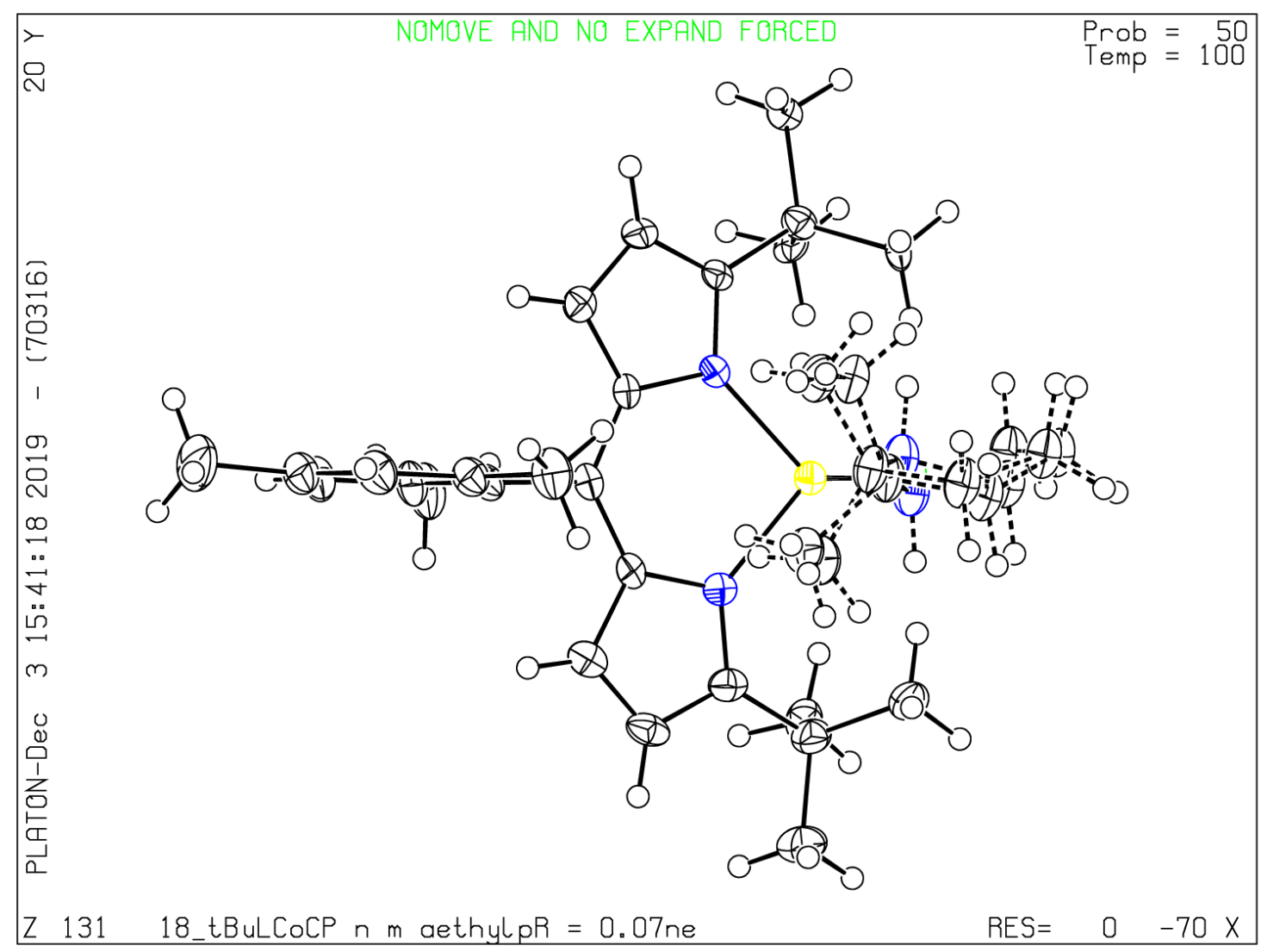




\section{checkCIF/PLATON report}

Structure factors have been supplied for datablock(s) 20_tBuLCoClnBu-imine

THIS REPORT IS FOR GUIDANCE ONLY. IF USED AS PART OF A REVIEW PROCEDURE FOR PUBLICATION, IT SHOULD NOT REPLACE THE EXPERTISE OF AN EXPERIENCED CRYSTALLOGRAPHIC REFEREE.

No syntax errors found. CIF dictionary Interpreting this report

\section{Datablock: 20_tBuLCoClnBu-imine}

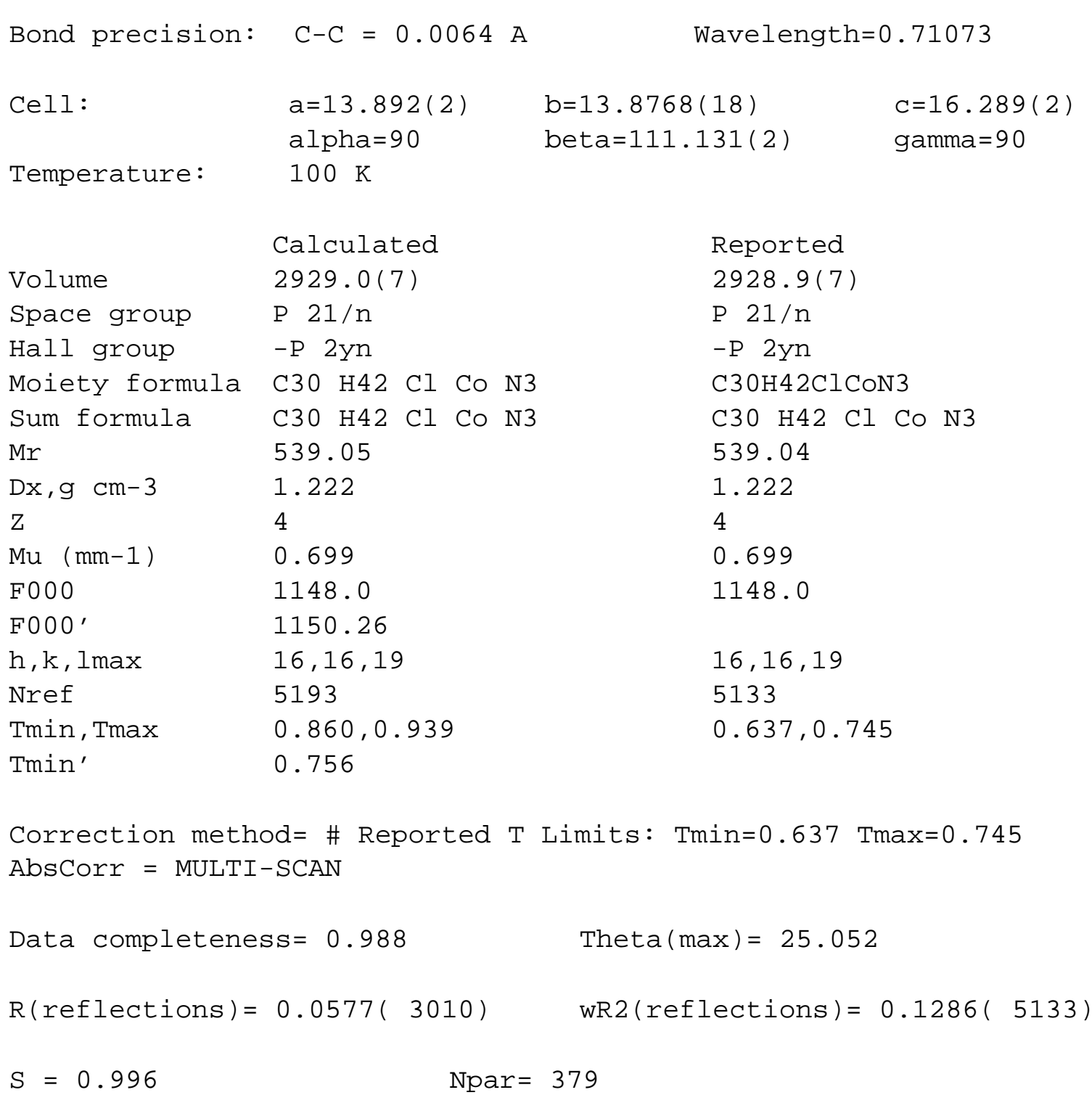

The following ALERTS were generated. Each ALERT has the format test-name_ALERT_alert-type_alert-level.

Click on the hyperlinks for more details of the test. 
Alert level $\mathrm{C}$

PLAT220_ALERT_2_C NonSolvent Resd 1 C Ueq(max) / Ueq(min) Range

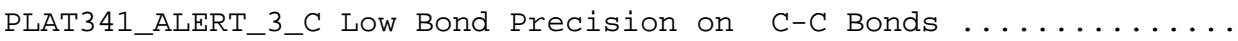

PLAT906_ALERT_3_C Large $K$ Value in the Analysis of Variance ......

PLAT911_ALERT_3_C Missing FCF Refl Between Thmin \& STh/L= 0.596

3.4 Ratio

0.00642 Ang.

3.036 Check

61 Report

\section{Alert level G}

PLAT002_ALERT_2_G Number of Distance or Angle Restraints on AtSite PLAT003_ALERT_2_G Number of Uiso or Uij Restrained non-H Atoms ...

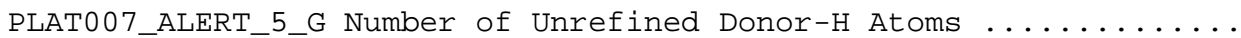
PLAT042_ALERT_1_G Calc. and Reported MoietyFormula Strings Differ PLAT171_ALERT_4_G The CIF-Embedded .res File Contains EADP Records PLAT175_ALERT_4_G The CIF-Embedded.res File Contains SAME Records PLAT176_ALERT_4_G The CIF-Embedded.res File Contains SADI Records PLAT178_ALERT_4_G The CIF-Embedded.res File Contains SIMU Records PLAT301_ALERT_3_G Main Residue Disorder ............ (Resd 1 ) PLAT413_ALERT_2_G Short Inter XH3 .. XHn H17A ..H29D . $-1 / 2+\mathrm{x}, 1 / 2-\mathrm{y},-1 / 2+\mathrm{z}=$

$\begin{aligned} & 16 \text { Note } \\ & 12 \text { Report } \\ & 2 \text { Report } \\ & \text { Please } \text { Check } \\ & 2 \text { Report } \\ & 1 \text { Report } \\ & 2 \text { Report } \\ & 1 \text { Report } \\ & 20 \% \text { Note } \\ & 1.93 \text { Ang. } \\ & 4565 \text { Check } \\ & 1 \text { Note } \\ & 157 \text { Note } \\ & 3.2 \text { Low } \\ & 0 \text { Info }\end{aligned}$

PLAT720_ALERT_4_G Number of Unusual/Non-Standard Labels ......... PLAT860_ALERT_3_G Number of Least-Squares Restraints ........... PLAT941_ALERT_3_G Average HKL Measurement Multiplicity .......... PLAT978_ALERT_2_G Number C-C Bonds with Positive Residual Density.

\footnotetext{
0 ALERT level $\mathbf{A}=$ Most likely a serious problem - resolve or explain

0 ALERT level $\mathbf{B}=\mathrm{A}$ potentially serious problem, consider carefully

4 ALERT level $\mathbf{C}=$ Check. Ensure it is not caused by an omission or oversight

14 ALERT level $\mathbf{G}=$ General information/check it is not something unexpected

1 ALERT type 1 CIF construction/syntax error, inconsistent or missing data

5 ALERT type 2 Indicator that the structure model may be wrong or deficient

6 ALERT type 3 Indicator that the structure quality may be low

5 ALERT type 4 Improvement, methodology, query or suggestion

1 ALERT type 5 Informative message, check
} 
It is advisable to attempt to resolve as many as possible of the alerts in all categories. Often the minor alerts point to easily fixed oversights, errors and omissions in your CIF or refinement strategy, so attention to these fine details can be worthwhile. In order to resolve some of the more serious problems it may be necessary to carry out additional measurements or structure refinements. However, the purpose of your study may justify the reported deviations and the more serious of these should normally be commented upon in the discussion or experimental section of a paper or in the "special_details" fields of the CIF. checkCIF was carefully designed to identify outliers and unusual parameters, but every test has its limitations and alerts that are not important in a particular case may appear. Conversely, the absence of alerts does not guarantee there are no aspects of the results needing attention. It is up to the individual to critically assess their own results and, if necessary, seek expert advice.

\section{Publication of your CIF in IUCr journals}

A basic structural check has been run on your CIF. These basic checks will be run on all CIFs submitted for publication in IUCr journals (Acta Crystallographica, Journal of Applied Crystallography, Journal of Synchrotron Radiation); however, if you intend to submit to Acta Crystallographica Section C or E or IUCrData, you should make sure that full publication checks are run on the final version of your CIF prior to submission.

\section{Publication of your CIF in other journals}

Please refer to the Notes for Authors of the relevant journal for any special instructions relating to CIF submission.

\section{PLATON version of 22/04/2020; check.def file version of 09/03/2020}


Datablock 20_tBuLCoClnBu-imine - ellipsoid plot

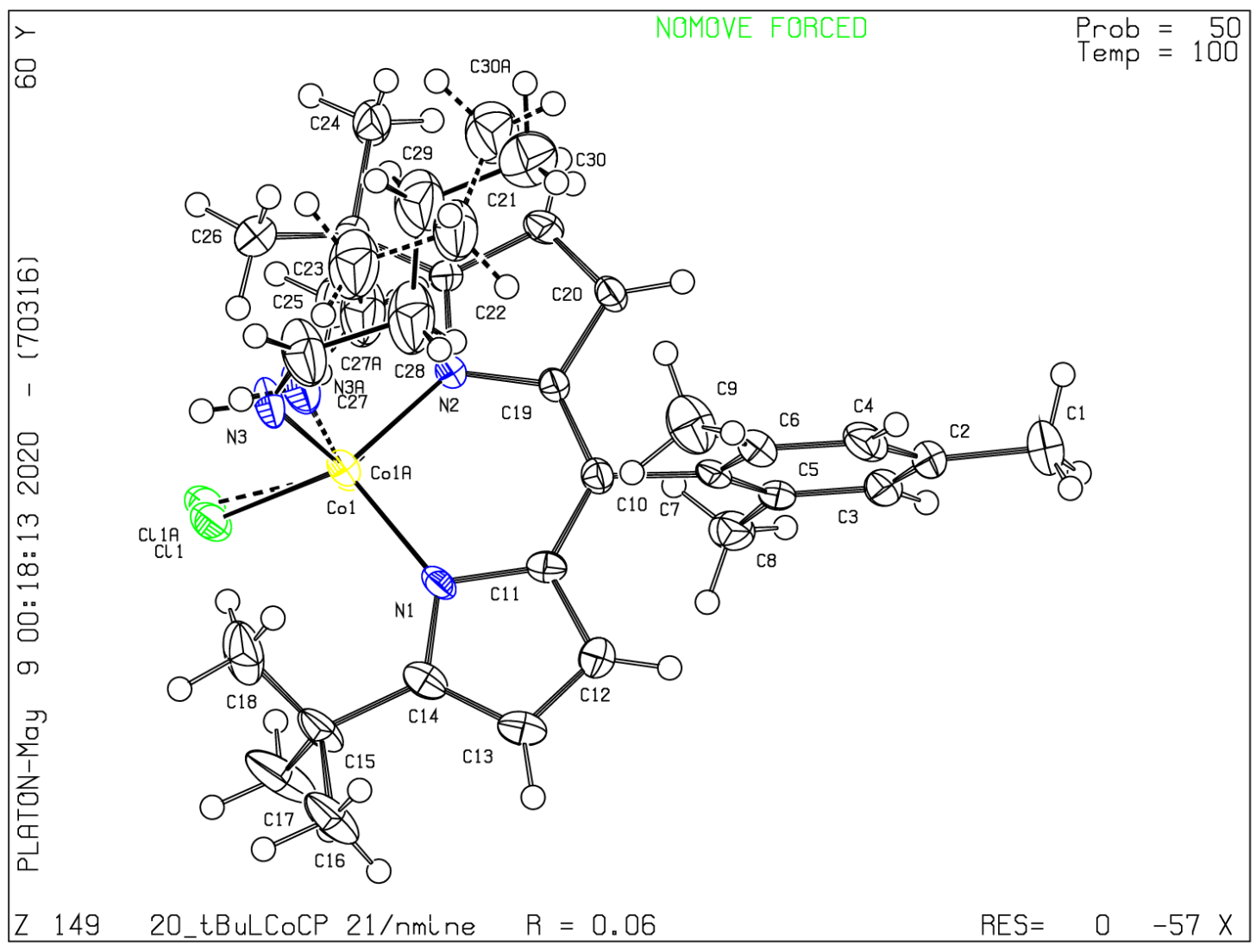




\section{checkCIF/PLATON report}

Structure factors have been supplied for datablock(s) 21_tBuLCoClnBuNH2

THIS REPORT IS FOR GUIDANCE ONLY. IF USED AS PART OF A REVIEW PROCEDURE FOR PUBLICATION, IT SHOULD NOT REPLACE THE EXPERTISE OF AN EXPERIENCED CRYSTALLOGRAPHIC REFEREE.

No syntax errors found. CIF dictionary Interpreting this report

\section{Datablock: 21_tBuLCoClnBuNH2}

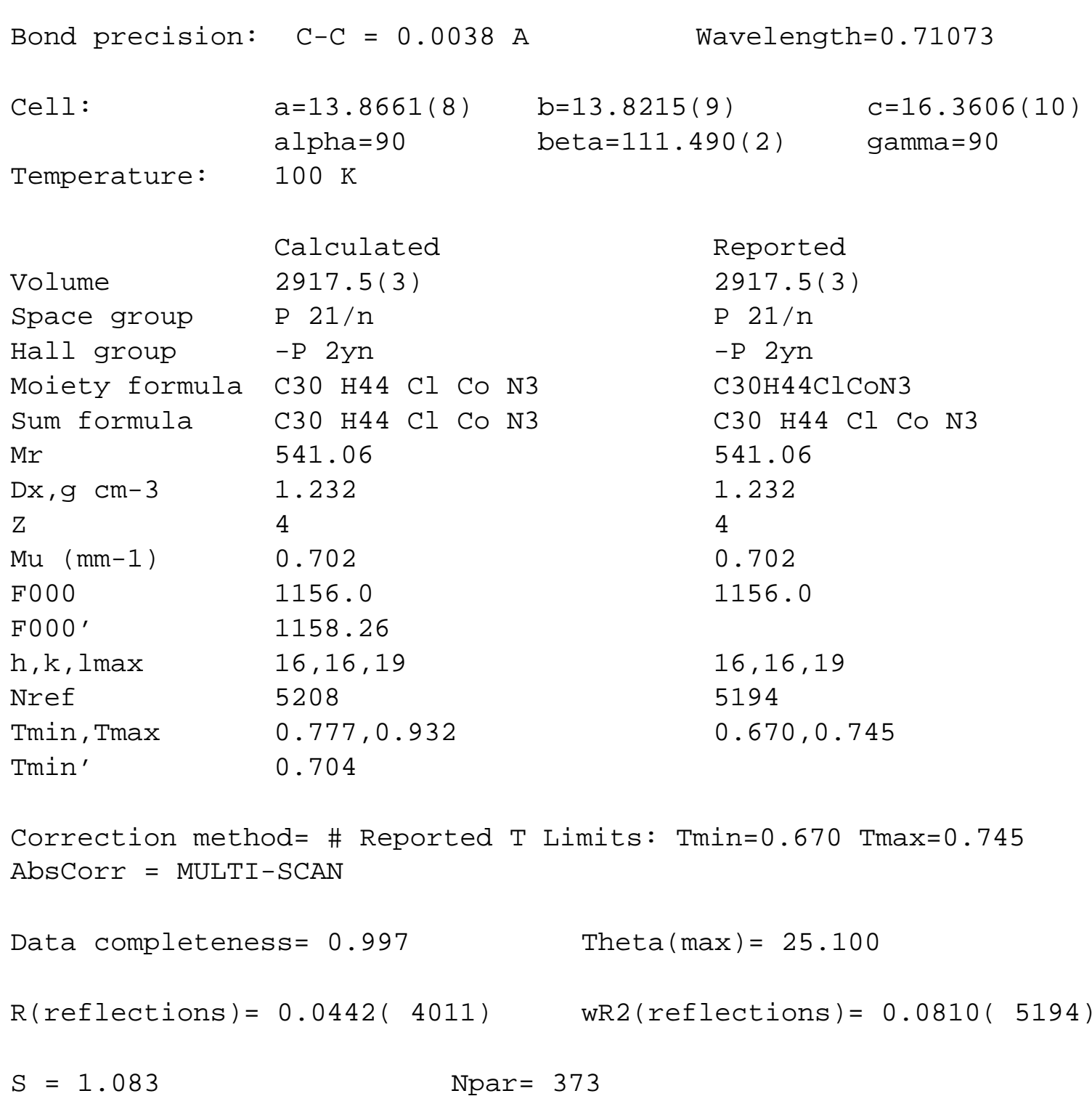

The following ALERTS were generated. Each ALERT has the format test-name_ALERT_alert-type_alert-level.

Click on the hyperlinks for more details of the test. 


\section{Alert level $\mathrm{C}$}

PLAT220_ALERT_2_C Non-Solvent Resd 1 C Ueq(max)/Ueq(min) Range PLAT420_ALERT_2_C D-H Without Acceptor N3 N PLAT906_ALERT_3_C Large $K$ Value in the Analysis of Variance ...... PLAT911_ALERT_3_C Missing FCF Refl Between Thmin \& STh/L= 0.597
3.2 Ratio

Please Check 3.909 Check

11 Report

\section{Alert level G}

PLAT002_ALERT_2_G Number of Distance or Angle Restraints on AtSite PLAT003_ALERT_2_G Number of Uiso or Uij Restrained non-H Atoms ... PLAT007_ALERT_5_G Number of Unrefined Donor-H Atoms ............ PLAT042_ALERT_1_G Calc. and Reported MoietyFormula Strings Differ PLAT175_ALERT_4_G The CIF-Embedded .res File Contains SAME Records PLAT176_ALERT_4_G The CIF-Embedded.res File Contains SADI Records PLAT178_ALERT_4_G The CIF-Embedded.res File Contains SIMU Records PLAT301_ALERT_3_G Main Residue Disorder...............Resd 1 ) PLAT720_ALERT_4_G Number of Unusual/Non-Standard Labels ......... PLAT860_ALERT_3_G Number of Least-Squares Restraints ........... PLAT909_ALERT_3_G Percentage of I>2sig(I) Data at Theta(Max) Still PLAT910_ALERT_3_G Missing \# of FCF Reflection(s) Below Theta(Min). PLAT978_ALERT_2_G Number C-C Bonds with Positive Residual Density.

0 ALERT level $\mathbf{A}=$ Most likely a serious problem - resolve or explain

0 ALERT level $\mathbf{B}=A$ potentially serious problem, consider carefully

4 ALERT level $\mathbf{C}=$ Check. Ensure it is not caused by an omission or oversight

13 ALERT level $\mathbf{G}=$ General information/check it is not something unexpected

1 ALERT type 1 CIF construction/syntax error, inconsistent or missing data

5 ALERT type 2 Indicator that the structure model may be wrong or deficient

6 ALERT type 3 Indicator that the structure quality may be low

4 ALERT type 4 Improvement, methodology, query or suggestion

1 ALERT type 5 Informative message, check 
It is advisable to attempt to resolve as many as possible of the alerts in all categories. Often the minor alerts point to easily fixed oversights, errors and omissions in your CIF or refinement strategy, so attention to these fine details can be worthwhile. In order to resolve some of the more serious problems it may be necessary to carry out additional measurements or structure refinements. However, the purpose of your study may justify the reported deviations and the more serious of these should normally be commented upon in the discussion or experimental section of a paper or in the "special_details" fields of the CIF. checkCIF was carefully designed to identify outliers and unusual parameters, but every test has its limitations and alerts that are not important in a particular case may appear. Conversely, the absence of alerts does not guarantee there are no aspects of the results needing attention. It is up to the individual to critically assess their own results and, if necessary, seek expert advice.

\section{Publication of your CIF in IUCr journals}

A basic structural check has been run on your CIF. These basic checks will be run on all CIFs submitted for publication in IUCr journals (Acta Crystallographica, Journal of Applied Crystallography, Journal of Synchrotron Radiation); however, if you intend to submit to Acta Crystallographica Section C or E or IUCrData, you should make sure that full publication checks are run on the final version of your CIF prior to submission.

\section{Publication of your CIF in other journals}

Please refer to the Notes for Authors of the relevant journal for any special instructions relating to CIF submission.

\section{PLATON version of 07/08/2019; check.def file version of 30/07/2019}


Datablock 21_tBuLCoClnBuNH2 - ellipsoid plot

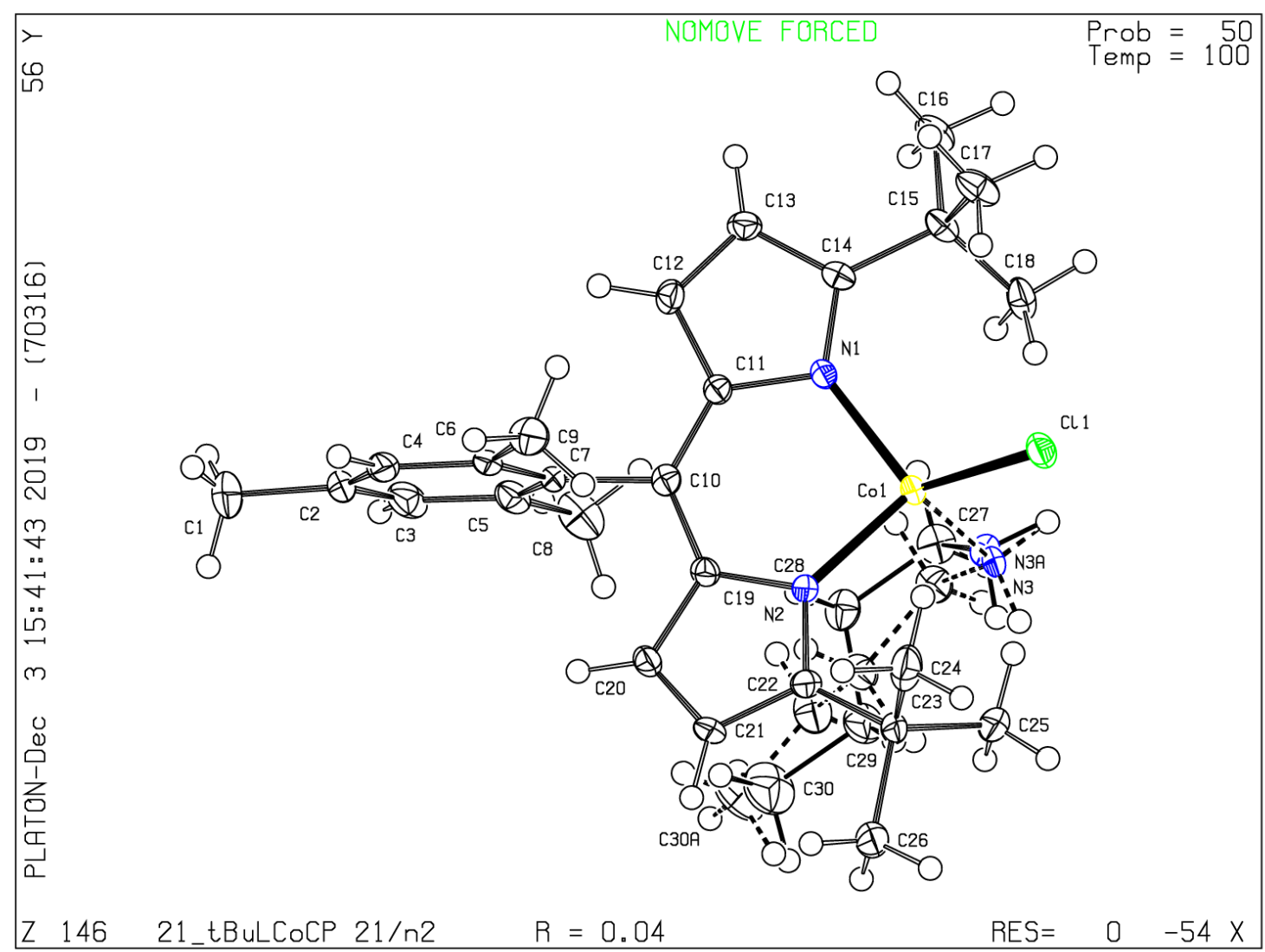




\section{checkCIF/PLATON report}

Structure factors have been supplied for datablock(s) 8-heat1

THIS REPORT IS FOR GUIDANCE ONLY. IF USED AS PART OF A REVIEW PROCEDURE FOR PUBLICATION, IT SHOULD NOT REPLACE THE EXPERTISE OF AN EXPERIENCED CRYSTALLOGRAPHIC REFEREE.

No syntax errors found. CIF dictionary Interpreting this report

\section{Datablock: 8-heat1}

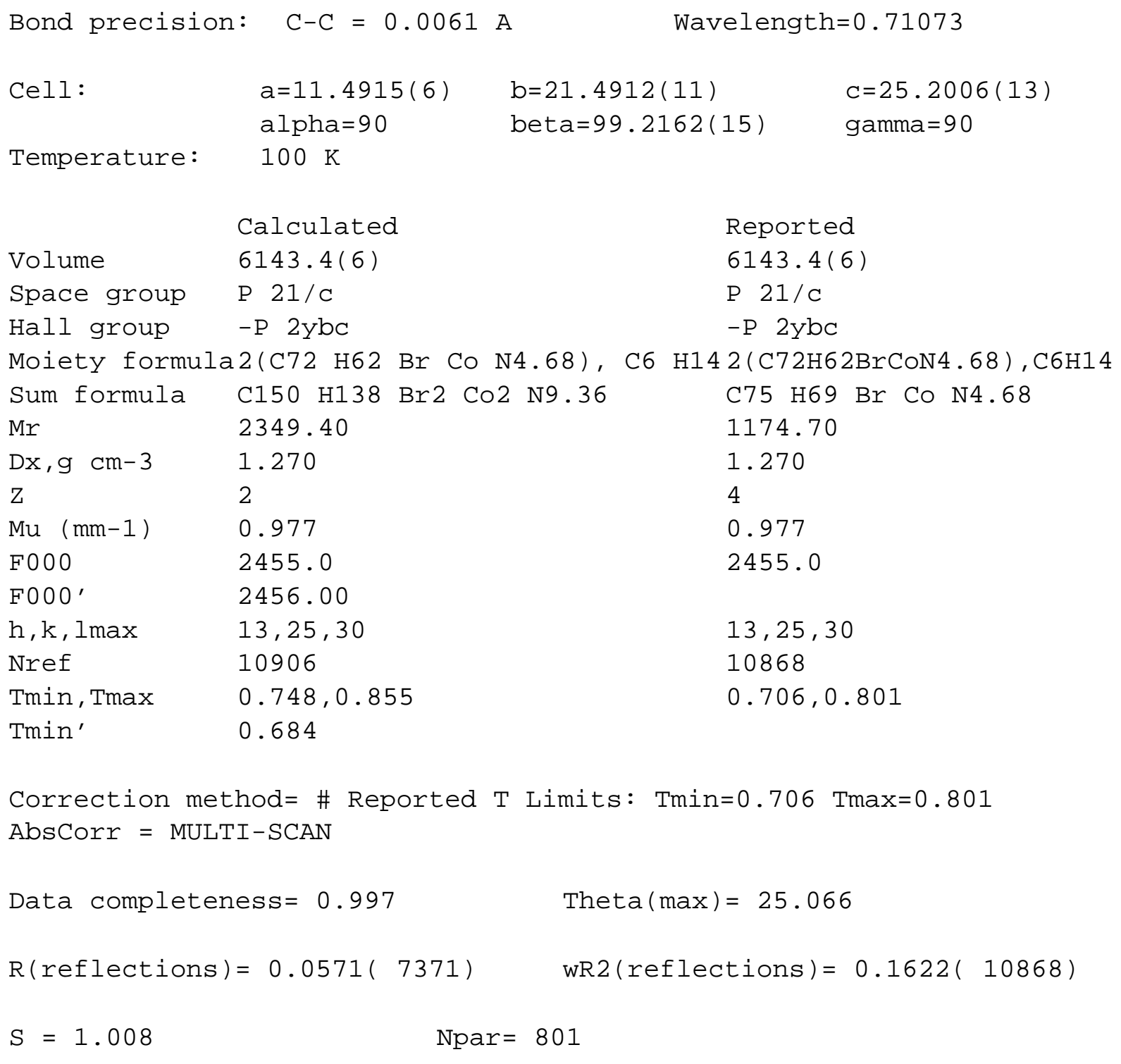

The following ALERTS were generated. Each ALERT has the format test-name_ALERT_alert-type_alert-level.

Click on the hyperlinks for more details of the test. 
Alert level $\mathrm{C}$

PLAT077_ALERT_4_C Unitcell Contains Non-integer Number of Atoms .. PLAT220_ALERT_2_C Non-Solvent Resd 1 C Ueq(max)/Ueq(min) Range PLAT221_ALERT_2_C Solv./Anion Resd 2 C Ueq (max)/Ueq(min) Range PLAT223_ALERT_4_C Solv./Anion Resd $2 \mathrm{H}$ Ueq(max)/Ueq(min) Range PLAT234_ALERT_4_C PLAT250_ALERT_2_C PLAT260_ALERT_2_C Large Hirshfeld Difference C26 --C27 . Large Average Ueq of Residue Including

Please Check 3.1 Ratio 4.6 Ratio

5.8 Ratio

0.17 Ang.

3.9 Note

0.184 Check

0.00605 Ang.

2.12 Ang.

3_666 Check

2.14 Ang.

1_655 Check

2.826 Check

35 Report

0 Info

PLAT911_ALERT_3_C PLAT978_ALERT_2_C Number C-C Bonds with Positive Residual Density.

$0.0 \dot{9}$

\section{Alert level G}

FORMU01_ALERT_1_G There is a discrepancy between the atom counts in the

_chemical_formula_sum and_chemical_formula_moiety. This is

usually due to the moiety formula being in the wrong format. Atom count from_chemical_formula_sum: C75 H69 Br1 Co1 N4.68

Atom count from_chemical_formula_moiety:C150 H138 Br2 Co2 N9.36

PLAT002_ALERT_2_G Number of Distance or Angle Restraints on AtSite PLAT003_ALERT_2_G Number of Uiso or Uij Restrained non-H Atoms ... PLAT042_ALERT_1_G Calc. and Reported MoietyFormula Strings Differ PLAT045_ALERT_1_G Calculated and Reported Z Differ by a Factor... PLAT083_ALERT_2_G SHELXL Second Parameter in WGHT Unusually Large PLAT171_ALERT_4_G The CIF-Embedded.res File Contains EADP Records PLAT172_ALERT_4_G The CIF-Embedded .res File Contains DFIX Records PLAT175_ALERT_4_G The CIF-Embedded .res File Contains SAME Records PLAT176_ALERT_4_G The CIF-Embedded.res File Contains SADI Records PLAT178_ALERT_4_G The CIF-Embedded.res File Contains SIMU Records PLAT187_ALERT_4_G The CIF-Embedded .res File Contains RIGU Records PLAT230 ALERT 2 G PLAT300_ALERT_4_G PLAT300_ALERT_4_G PLAT 300 ALERT 4 PLAT300_ALERT_4_G PLAT300_ALERT_4_G PLAT300_ALERT_4_G PLAT300_ALERT 4 PLAT300_ALERT_4_G PLAT300_ALERT_4_G PLAT300_ALERT_4_G PLAT300 ALERT $4 \mathrm{G}$ PLAT300_ALERT_4_G PLAT300_ALERT_4_G PLAT300_ALERT_4_G PLAT300_ALERT 4_G PLAT300_ALERT_4_G PLAT300_ALERT_4_G PLAT300_ALERT 4 G PLAT300_ALERT_4_G PLAT300_ALERT_4_G PLAT301_ALERT_3_G PLAT302 ALERT 4 G PLAT304_ALERT_4_G PLAT411_ALERT_2_G
Hirshfeld Test Diff for

Atom Site Occupancy of C1S Atom Site Occupancy of C2S Atom Site Occupancy of C3S Atom Site Occupancy of C4S Atom Site Occupancy of C5S Atom Site Occupancy of C6S Atom Site Occupancy of H1SA Atom Site Occupancy of H1SB Atom Site Occupancy of H1SC Atom Site Occupancy of H2SA Atom Site Occupancy of H2SB Atom Site Occupancy of H3SA Atom Site Occupancy of H3SB Atom Site Occupancy of H4SA Atom Site Occupancy of H4SB Atom Site Occupancy of H5SA Atom Site Occupancy of H5SB Atom Site Occupancy of H6SA Atom Site Occupancy of H6SB Atom Site Occupancy of H6SC N3 $--\mathrm{N} 4$ Constrained at Constrained at Constrained at Constrained at Constrained at Constrained at Constrained at Constrained at Constrained at Constrained at Constrained at Constrained at Constrained at Constrained at Constrained at Constrained at Constrained at Constrained at Constrained at Constrained at Main Residue Disorder ............ (Resd 1) Anion/Solvent/Minor-Residue Disorder (Resd 2 ) Non-Integer Number of Atoms in ..... Resd 1 Short Inter H...H Contact H2SB ..H28

$$
1-x,-1 / 2+y, 3 / 2-z=
$$

20 Note

6 Report

Please Check

0.50 Check

11.75 Why ?

9 Report

1 Report

2 Report

2 Report

1 Report

1 Report

$6.3 \mathrm{s.u}$.

0.5 Check

0.5 Check

0.5 Check

0.5 Check

0.5 Check

0.5 Check

0.5 Check

0.5 Check

0.5 Check

0.5 Check

0.5 Check

0.5 Check

0.5 Check

0.5 Check

0.5 Check

0.5 Check

0.5 Check

0.5 Check

0.5 Check

0.5 Check

$14 \%$ Note

$100 \%$ Note

140.68 Check

2.06 Ang.

2_646 Check 


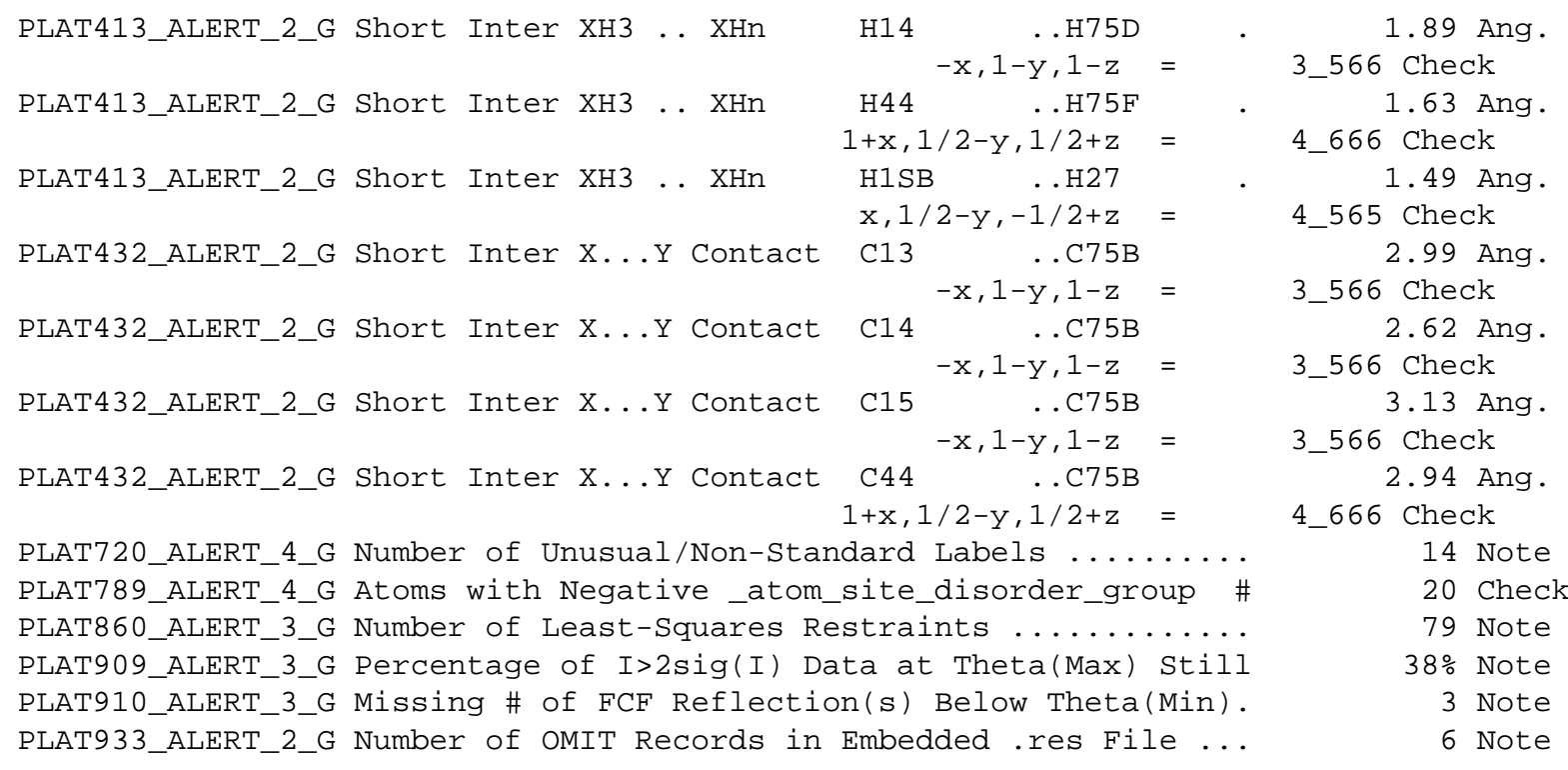

0 ALERT level $\mathbf{A}=$ Most likely a serious problem - resolve or explain

0 ALERT level $\mathbf{B}=\mathrm{A}$ potentially serious problem, consider carefully

13 ALERT level $\mathbf{C}=$ Check. Ensure it is not caused by an omission or oversight

50 ALERT level $\mathbf{G}=$ General information/check it is not something unexpected

3 ALERT type 1 CIF construction/syntax error, inconsistent or missing data

20 ALERT type 2 Indicator that the structure model may be wrong or deficient

7 ALERT type 3 Indicator that the structure quality may be low

33 ALERT type 4 Improvement, methodology, query or suggestion

0 ALERT type 5 Informative message, check 
It is advisable to attempt to resolve as many as possible of the alerts in all categories. Often the minor alerts point to easily fixed oversights, errors and omissions in your CIF or refinement strategy, so attention to these fine details can be worthwhile. In order to resolve some of the more serious problems it may be necessary to carry out additional measurements or structure refinements. However, the purpose of your study may justify the reported deviations and the more serious of these should normally be commented upon in the discussion or experimental section of a paper or in the "special_details" fields of the CIF. checkCIF was carefully designed to identify outliers and unusual parameters, but every test has its limitations and alerts that are not important in a particular case may appear. Conversely, the absence of alerts does not guarantee there are no aspects of the results needing attention. It is up to the individual to critically assess their own results and, if necessary, seek expert advice.

\section{Publication of your CIF in IUCr journals}

A basic structural check has been run on your CIF. These basic checks will be run on all CIFs submitted for publication in IUCr journals (Acta Crystallographica, Journal of Applied Crystallography, Journal of Synchrotron Radiation); however, if you intend to submit to Acta Crystallographica Section C or E or IUCrData, you should make sure that full publication checks are run on the final version of your CIF prior to submission.

\section{Publication of your CIF in other journals}

Please refer to the Notes for Authors of the relevant journal for any special instructions relating to CIF submission.

\section{PLATON version of 07/08/2019; check.def file version of 30/07/2019}




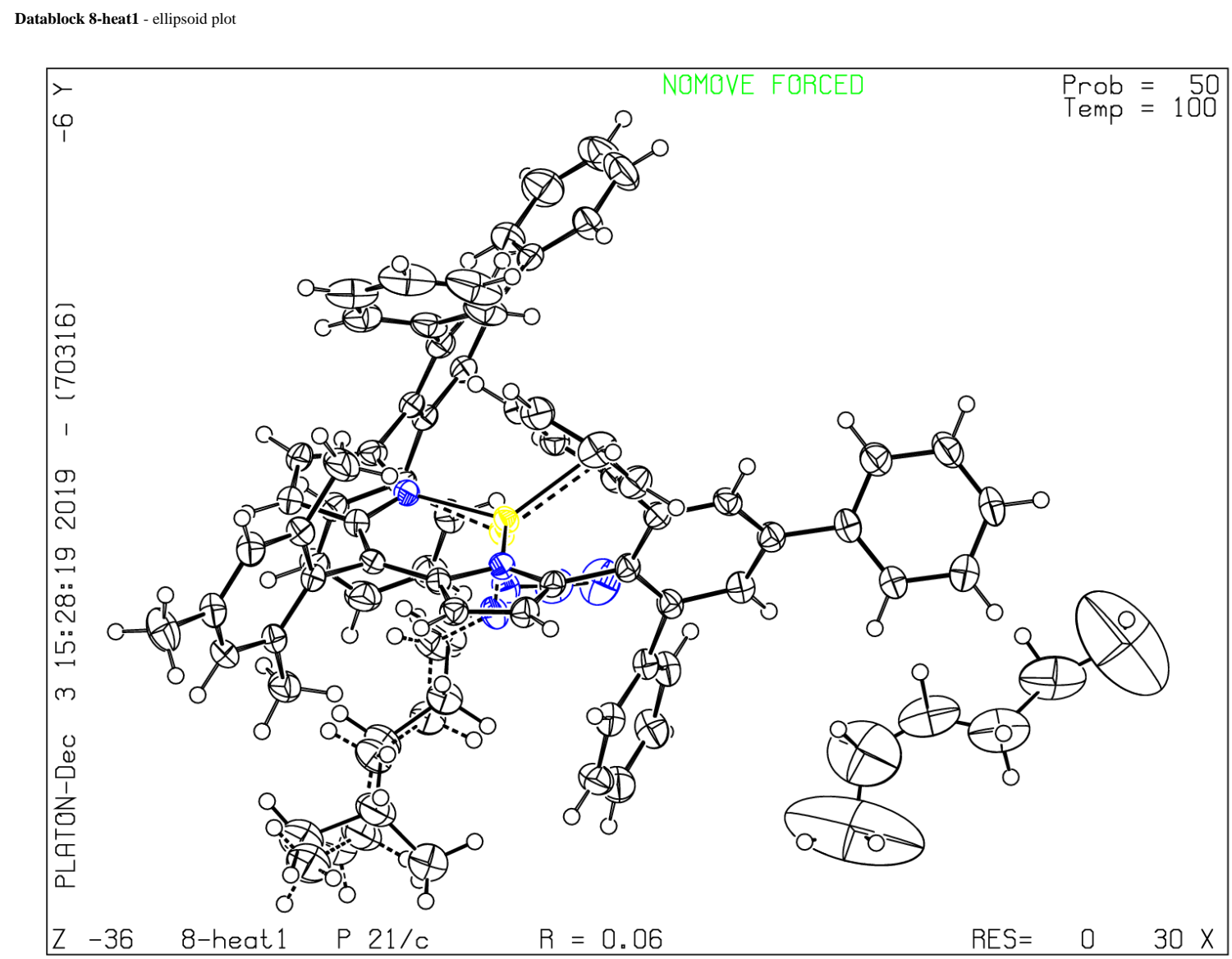




\section{checkCIF/PLATON report}

Structure factors have been supplied for datablock(s) 8-heat2

THIS REPORT IS FOR GUIDANCE ONLY. IF USED AS PART OF A REVIEW PROCEDURE FOR PUBLICATION, IT SHOULD NOT REPLACE THE EXPERTISE OF AN EXPERIENCED CRYSTALLOGRAPHIC REFEREE.

No syntax errors found. CIF dictionary Interpreting this report

\section{Datablock: 8-heat2}

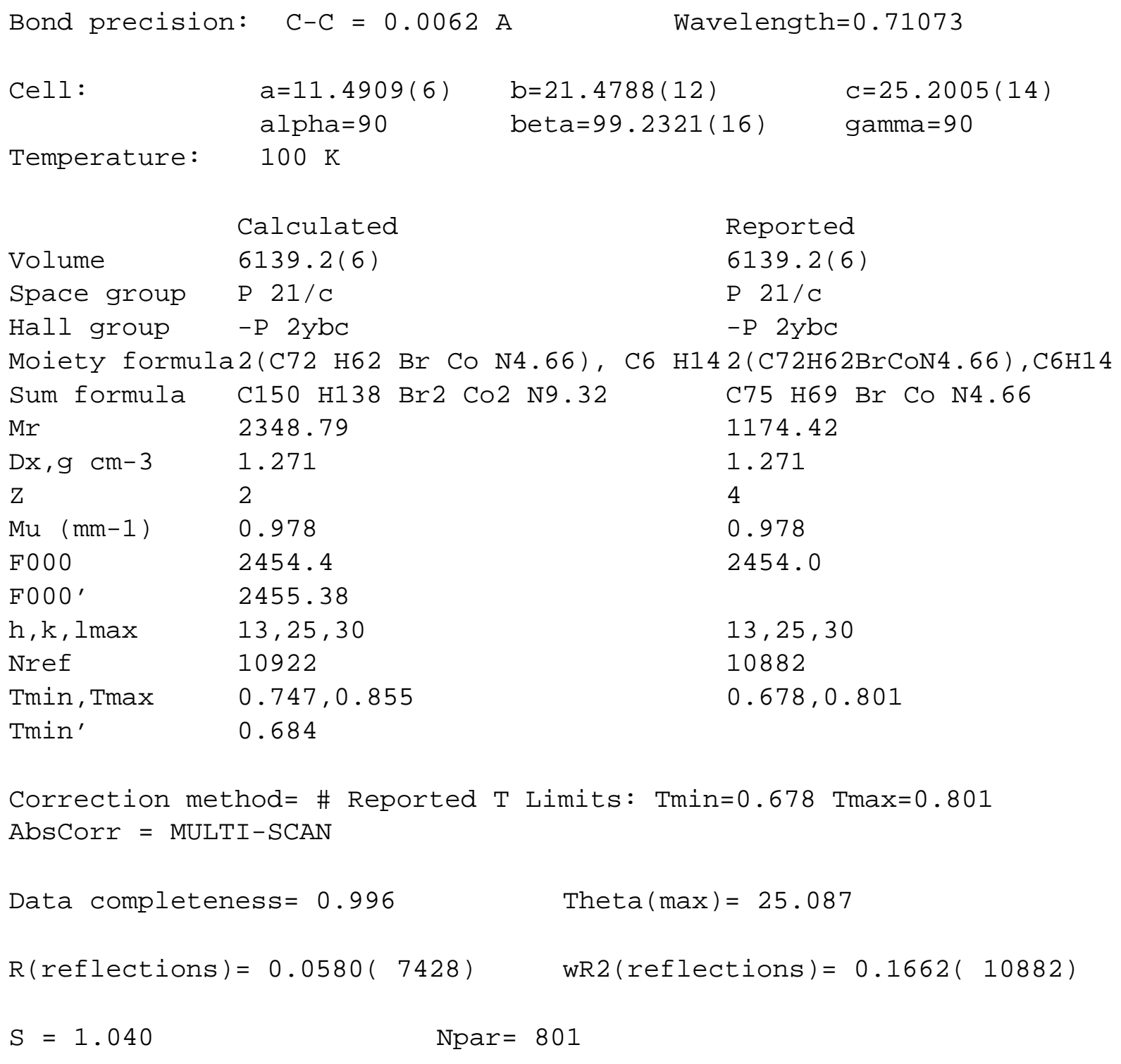

The following ALERTS were generated. Each ALERT has the format test-name_ALERT_alert-type_alert-level.

Click on the hyperlinks for more details of the test. 
Alert level $\mathrm{C}$

PLAT077_ALERT_4_C Unitcell Contains Non-integer Number of Atoms .. PLAT221_ALERT_2_C Solv./Anion Resd 2 C Ueq(max)/Ueq(min) Range PLAT223_ALERT_4_C Solv./Anion Resd 2 H Ueq (max)/Ueq(min) Range PLAT234 ALERT 4 PLAT234_ALERT_4_C PLAT250_ALERT_2_C PLAT260_ALERT_2_C Large Hirshfeld Difference C26 --C27 . Large Hirshfeld Difference C73 --C74 . Large U3/U1 Ratio for Average U(i,j) Tensor .... Large Average Ueq of Residue Including C1S

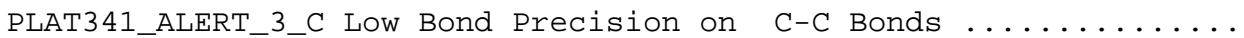
PLAT 411_ALERT_2_C Short Inter H...H Contact H17 $\ldots$ H17 . $1-\mathrm{x}, 1-\mathrm{y}, 1-\mathrm{z}=$ PLAT906_ALERT_3_C Large $K$ Value in the Analysis of Variance ...... PLAT911_ALERT_3_C Missing FCF Refl Between Thmin \& STh/L= 0.597 PLAT978_ALERT_2_C Number C-C Bonds with Positive Residual Density.

$$
\begin{aligned}
\text { Please Check } \\
4.6 \text { Ratio } \\
5.7 \text { Ratio } \\
0.17 \text { Ang. } \\
0.17 \text { Ang. } \\
3.8 \text { Note } \\
0.188 \text { Check } \\
0.00622 \text { Ang. } \\
2.11 \text { Ang. } \\
3 \text { 666 Check } \\
2.876 \text { Check } \\
37 \text { Report } \\
0 \text { Info }
\end{aligned}
$$

\section{Alert level G}

FORMU01_ALERT_1_G There is a discrepancy between the atom counts in the _chemical_formula_sum and_chemical_formula_moiety. This is usually due to the moiety formula being in the wrong format. Atom count from_chemical_formula_sum: C75 H69 Br1 Co1 N4.66 Atom count from _chemical_formula_moiety:C150 H138 Br2 Co2 N9.32

PLAT002_ALERT_2_G Number of Distance or Angle Restraints on AtSite PLAT003_ALERT_2_G Number of Uiso or Uij Restrained non-H Atoms ... PLAT042_ALERT_1_G Calc. and Reported MoietyFormula Strings Differ PLAT045_ALERT_1_G Calculated and Reported Z Differ by a Factor... PLAT068_ALERT_1_G Reported F000 Differs from Calcd (or Missing)... PLAT083_ALERT_2_G SHELXL Second Parameter in WGHT Unusually Large PLAT171_ALERT_4_G The CIF-Embedded.res File Contains EADP Records PLAT172_ALERT_4_G The CIF-Embedded.res File Contains DFIX Records PLAT175_ALERT_4_G The CIF-Embedded.res File Contains SAME Records PLAT176_ALERT_4_G The CIF-Embedded.res File Contains SADI Records PLAT178_ALERT_4_G The CIF-Embedded.res File Contains SIMU Records PLAT187_ALERT_4_G The CIF-Embedded.res File Contains RIGU Records PLAT230 ALERT 2 G PLAT300 ALERT 4 PLAT300_ALERT_4_G PLAT300_ALERT_4_G PLAT300_ALERT_4_G PLAT300_ALERT_4_G PLAT300_ALERT_4_G PLAT300_ALERT_4_G PLAT300 ALERT 4 G PLAT300_ALERT_4_G PLAT300_ALERT_4_G PLAT300_ALERT_4_G PLAT300_ALERT_4_G PLAT300_ALERT_4_G PLAT300_ALERT_4_G PLAT300_ALERT_4_G PLAT300_ALERT 4_G PLAT300_ALERT_4_G PLAT300_ALERT_4_G PLAT300 ALERT 4 G PLAT300_ALERT_4_G PLAT301_ALERT_3_G PLAT302_ALERT_4_G PLAT304_ALERT_4_G PLAT 411_ALERT_2_G Hirshfeld Test Diff for N3 Atom Site Occupancy of C1S Atom Site Occupancy of $\mathrm{C} 2 \mathrm{~S}$ Atom Site Occupancy of C3S Atom Site Occupancy of C4S Atom Site Occupancy of C5S Atom Site Occupancy of C6S Atom Site Occupancy of H1SA Atom Site Occupancy of H1SB Atom Site Occupancy of H1SC Atom Site Occupancy of H2SA Atom Site Occupancy of H2SB Atom Site Occupancy of H3SA Atom Site Occupancy of H3SB Atom Site Occupancy of H4SA Atom Site Occupancy of H4SB Atom Site Occupancy of H5SA Atom Site Occupancy of H5SB Atom Site Occupancy of H6SA Atom Site Occupancy of H6SB Atom Site Occupancy of H6SC Main Residue Disorder .............Resd 1) Anion/Solvent/Minor-Residue Disorder (Resd 2) Non-Integer Number of Atoms in ...... Resd 1 Short Inter H...H Contact H2SB ..H28

$$
\begin{array}{cc}
1-x,-1 / 2+y, & 3 / 2-z \\
H 14 & \cdots H 75 D
\end{array}=
$$$$
\text { H14 } \quad \text {. H } 75 \text { D }
$$
$\mathrm{H} 14$
.. H $75 \mathrm{D}$

Constrained at Constrained at trained at Constrained at Constrained at Constrained at Constrained at Constrained at trained at ined at Constrained at Constrained at Constrained at Constrained at Constrained at Constrained at Constrained at

PLAT413_ALERT_2_G Short Inter XH3 .. XHn 


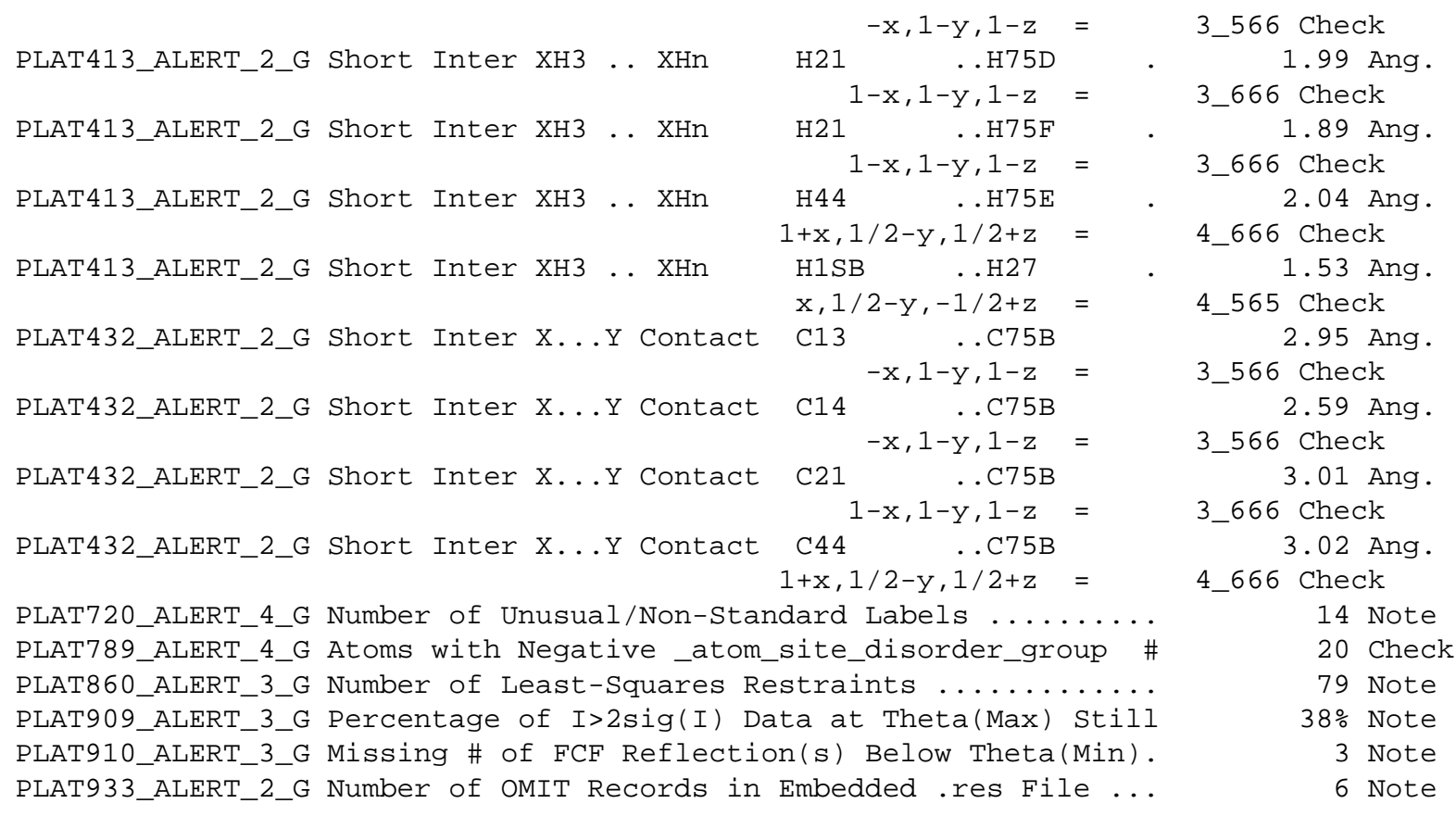

0 ALERT level $\mathbf{A}=$ Most likely a serious problem - resolve or explain

0 ALERT level $\mathbf{B}=\mathrm{A}$ potentially serious problem, consider carefully

12 ALERT level $\mathbf{C}=$ Check. Ensure it is not caused by an omission or oversight

53 ALERT level $\mathbf{G}=$ General information/check it is not something unexpected

4 ALERT type 1 CIF construction/syntax error, inconsistent or missing data

20 ALERT type 2 Indicator that the structure model may be wrong or deficient

7 ALERT type 3 Indicator that the structure quality may be low

34 ALERT type 4 Improvement, methodology, query or suggestion

0 ALERT type 5 Informative message, check 
It is advisable to attempt to resolve as many as possible of the alerts in all categories. Often the minor alerts point to easily fixed oversights, errors and omissions in your CIF or refinement strategy, so attention to these fine details can be worthwhile. In order to resolve some of the more serious problems it may be necessary to carry out additional measurements or structure refinements. However, the purpose of your study may justify the reported deviations and the more serious of these should normally be commented upon in the discussion or experimental section of a paper or in the "special_details" fields of the CIF. checkCIF was carefully designed to identify outliers and unusual parameters, but every test has its limitations and alerts that are not important in a particular case may appear. Conversely, the absence of alerts does not guarantee there are no aspects of the results needing attention. It is up to the individual to critically assess their own results and, if necessary, seek expert advice.

\section{Publication of your CIF in IUCr journals}

A basic structural check has been run on your CIF. These basic checks will be run on all CIFs submitted for publication in IUCr journals (Acta Crystallographica, Journal of Applied Crystallography, Journal of Synchrotron Radiation); however, if you intend to submit to Acta Crystallographica Section C or E or IUCrData, you should make sure that full publication checks are run on the final version of your CIF prior to submission.

\section{Publication of your CIF in other journals}

Please refer to the Notes for Authors of the relevant journal for any special instructions relating to CIF submission.

\section{PLATON version of 07/08/2019; check.def file version of 30/07/2019}


Datablock 8-heat2 - ellipsoid plot

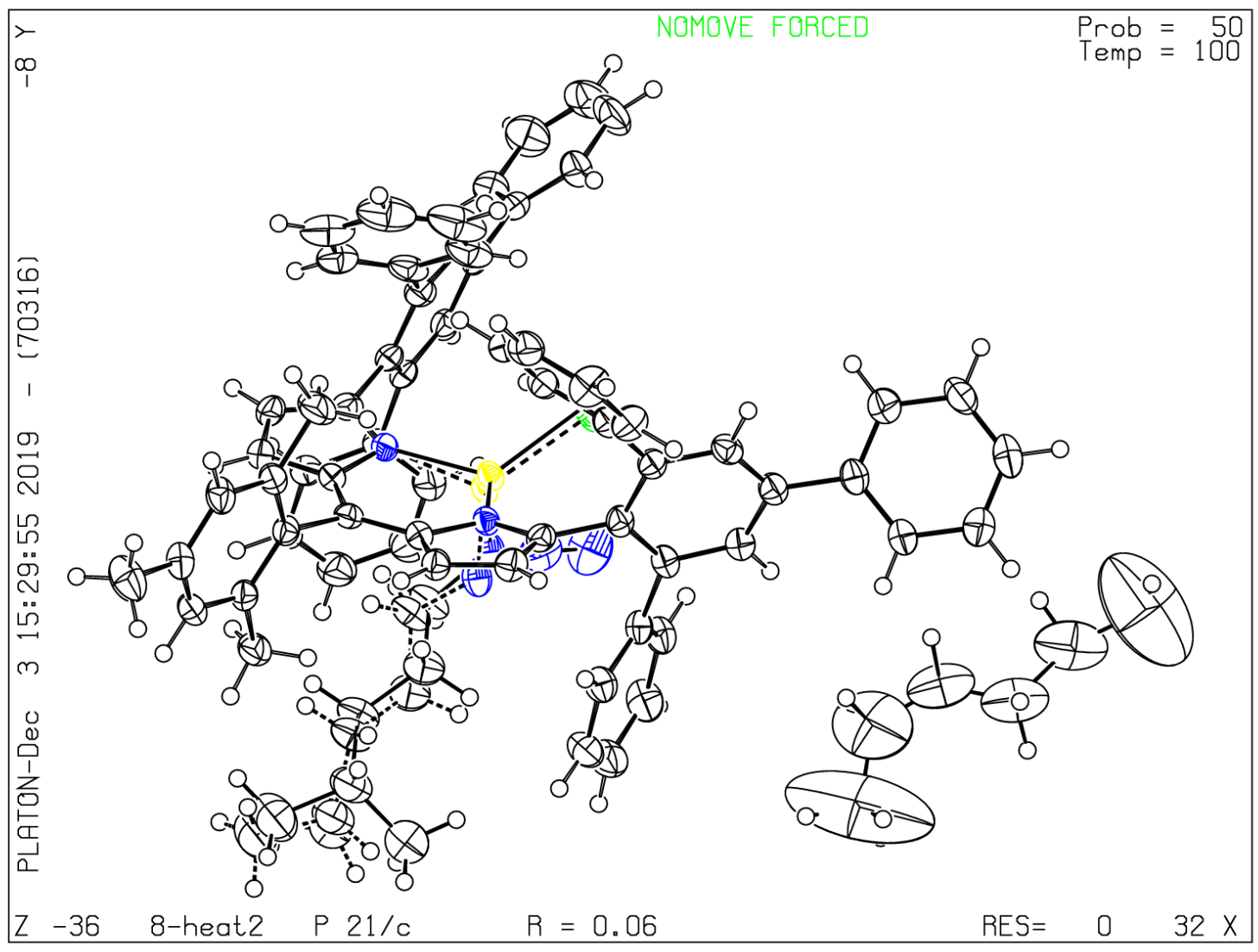




\section{checkCIF/PLATON report}

Structure factors have been supplied for datablock(s) 8-heat3

THIS REPORT IS FOR GUIDANCE ONLY. IF USED AS PART OF A REVIEW PROCEDURE FOR PUBLICATION, IT SHOULD NOT REPLACE THE EXPERTISE OF AN EXPERIENCED CRYSTALLOGRAPHIC REFEREE.

No syntax errors found. CIF dictionary Interpreting this report

\section{Datablock: 8-heat3}

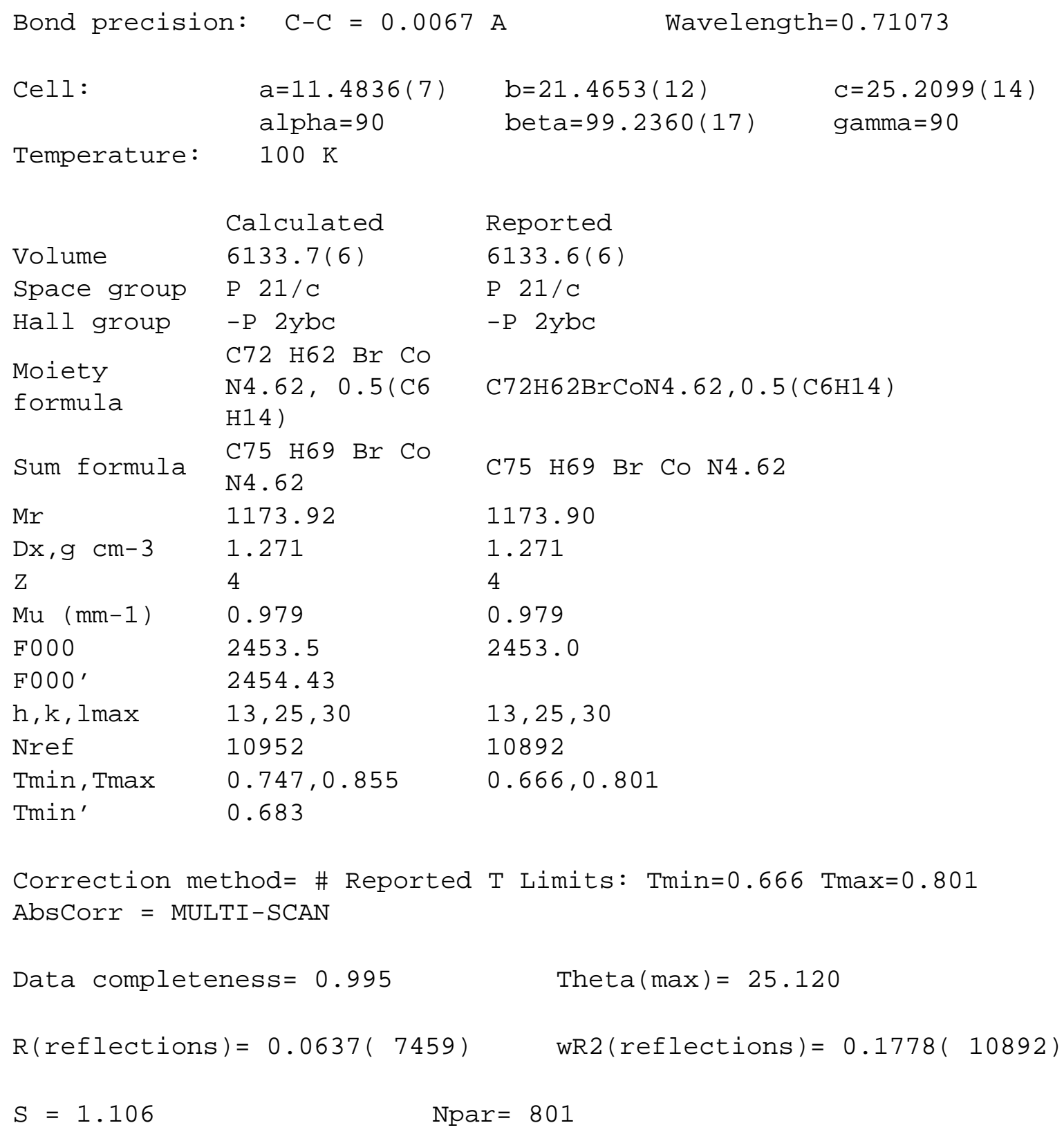


The following ALERTS were generated. Each ALERT has the format

test-name_ALERT_alert-type_alert-level.

Click on the hyperlinks for more details of the test.

\section{Alert level $C$}

PLAT077_ALERT_4_C Unitcell Contains Non-integer Number of Atoms .. PLAT220_ALERT_2_C Non-Solvent Resd 1 C Ueq(max)/Ueq(min) Range PLAT223_ALERT_4_C Solv./Anion Resd 2 H Ueq (max)/Ueq(min) Range PLAT234_ALERT_4_C PLAT234_ALERT_4_C PLAT250_ALERT_2_C PLAT260_ALERT_2_C PLAT341_ALERT_3_C PLAT 411_ALERT_2_C Large Hirshfeld Difference C26 Large Hirshfeld Difference C74 $--\mathrm{C} 27$ Large U3/U1 Ratio for Average U(i,j) Tensor .... Large Average Ueq of Residue Including $\mathrm{C} 1 \mathrm{~S}$

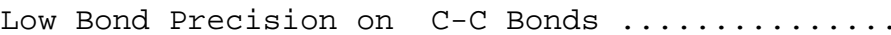
Short Inter H...H Contact $\mathrm{H} 1$

PLAT413_ALERT_2_C Short Inter XH3 .. XHn

$$
\text { H1 } 7
$$$$
\text { ..H17 }
$$

H2 6

$$
\begin{array}{r}
1-x, 1-y, 1-z= \\
. .465 B
\end{array}
$$$$
1+\mathrm{x}, \mathrm{y}, \mathrm{z}
$$

PLAT906_ALERT_3

PLAT911_ALERT_3_C

Lar Missing FCF Refl Between Thmin \& STh/L=
Variance (1)

\section{Alert level $\mathbf{G}$}

PLAT002_ALERT_2_G Number of Distance or Angle Restraints on Atsite PLAT003_ALERT_2_G Number of Uiso or Uij Restrained non-H Atoms ... PLAT042_ALERT_1_G Calc. and Reported MoietyFormula Strings Differ PLAT068_ALERT_1_G Reported F000 Differs from Calcd (or Missing)... PLAT083_ALERT_2_G SHELXL Second Parameter in WGHT Unusually Large PLAT171_ALERT_4_G The CIF-Embedded.res File Contains EADP Records PLAT172_ALERT_4_G The CIF-Embedded .res File Contains DFIX Records PLAT175_ALERT_4_G The CIF-Embedded.res File Contains SAME Records PLAT176_ALERT_4_G The CIF-Embedded.res File Contains SADI Records PLAT178_ALERT_4_G The CIF-Embedded .res File Contains SIMU Records PLAT187_ALERT_4_G The CIF-Embedded.res File Contains RIGU Records PLAT230 ALERT 2 G Hirshfeld Test Diff for N3 PLAT300_ALERT_4_G Atom Site Occupancy of C1S Constrained at PLAT300_ALERT_4_G Atom Site Occupancy of C2S PLAT300_ALERT_4_G Atom Site Occupancy of C3S PLAT300_ALERT_4_G Atom Site Occupancy of C4S PLAT300_ALERT_4_G Atom Site Occupancy of C5S PLAT300_ALERT_4_G Atom Site Occupancy of C6S PLAT300_ALERT_4_G Atom Site Occupancy of H1SA PLAT300_ALERT_4_G Atom Site Occupancy of H1SB PLAT300_ALERT_4_G Atom Site Occupancy of H1SC PLAT300_ALERT_4_G Atom Site Occupancy of H2SA PLAT300_ALERT_4_G Atom Site Occupancy of H2SB PLAT300_ALERT_4_G Atom Site Occupancy of H3SA PLAT300_ALERT_4_G Atom Site Occupancy of H3SB PLAT300_ALERT_4_G Atom Site Occupancy of H4SA PLAT300_ALERT_4_G Atom Site Occupancy of H4SB PLAT300_ALERT_4_G Atom Site Occupancy of H5SA PLAT300_ALERT_4_G Atom Site Occupancy of H5SB PLAT300_ALERT_4_G Atom Site Occupancy of H6SA PLAT300_ALERT_4_G Atom Site Occupancy of H6SB PLAT300_ALERT_4_G Atom Site Occupancy of H6SC PLAT 301_ALERT 3_G PLAT302_ALERT_4_G PLAT304_ALERT_4_G PLAT 411_ALERT_2_G
Constrained at

Constrained at

Constrained at

Constrained at

Constrained at

Constrained at

Constrained at

Constrained at

Constrained at

constrained at

Constrained at

Constrained at

Constrained at

Constrained at

Constrained at

Constrained at

Constrained at

$3 / 2-z=$ Anion/Solvent/Minor-Residue Disorder (Resd 2) Non-Integer Number of Atoms in ...... Resd 1 Short Inter $\mathrm{H} \ldots \mathrm{H}$ Contact H2SB $\quad . \mathrm{H} 28$

$$
1-x,-1 / 2+y, 3 / 2-z=
$$

Please Check 3.3 Ratio

5.0 Ratio

0.17 Ang.

0.18 Ang.

3.0 Note

0.179 Check

0.00673 Ang.

2.12 Ang.

3_666 Check

2.14 Ang.

1_655 Check

3.734 Check

56 Report
20 Note

6 Report

Please Check

Please Check

11.75 Why ?

9 Report

1 Report

2 Report

2 Report

1 Report

1 Report

$10.2 \mathrm{s.u}$.

0.5 Check

0.5 Check

0.5 Check

0.5 Check

0.5 Check

0.5 Check

0.5 Check

0.5 Check

0.5 Check

0.5 Check

0.5 Check

0.5 Check

0.5 Check

0.5 Check

0.5 Check

0.5 Check

0.5 Check

0.5 Check

0.5 Check

0.5 Check

$14 \%$ Note

$100 \%$ Note

140.62 Check

$2.03 \mathrm{Ang}$.

2_646 Check 


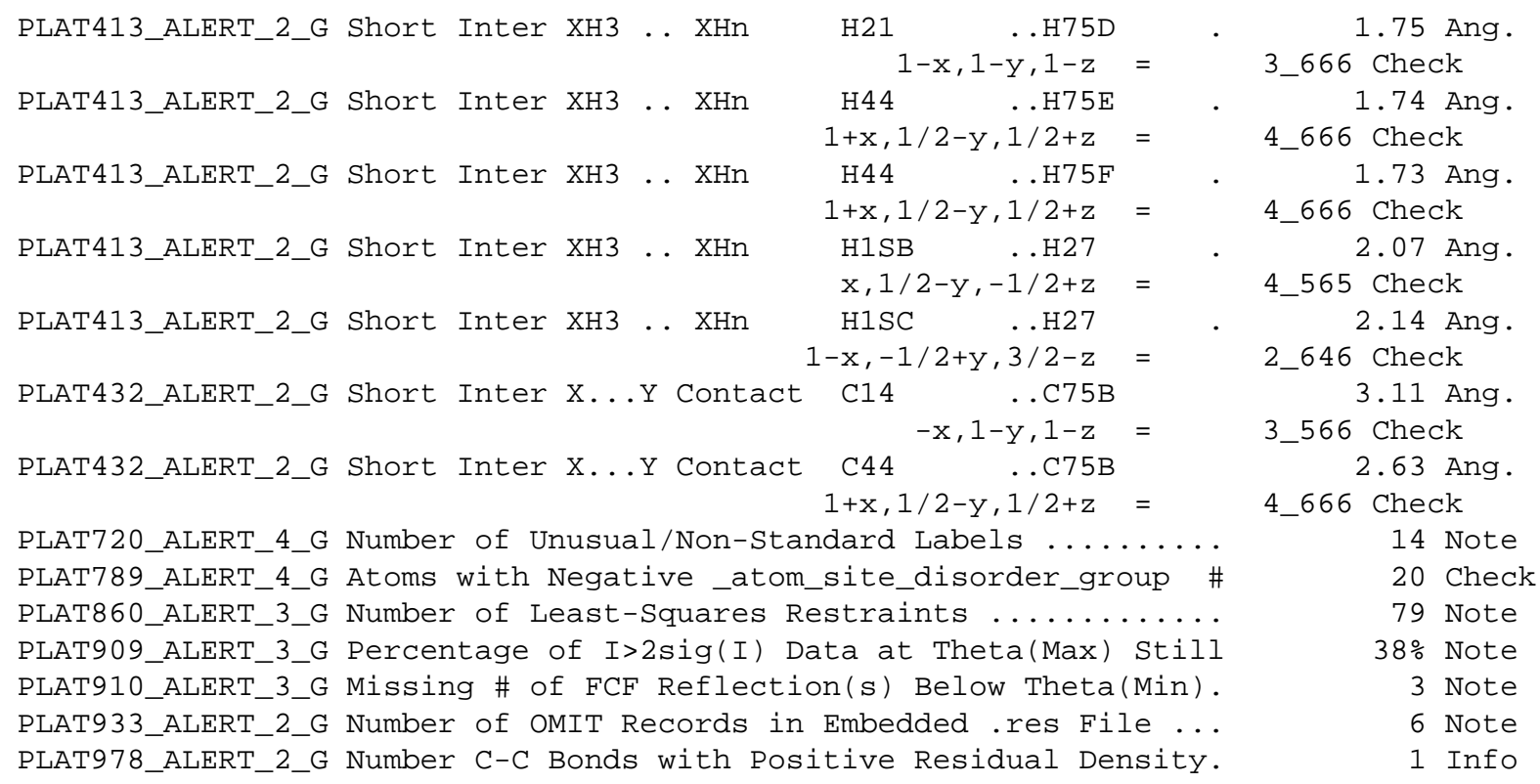

0 ALERT level $\mathbf{A}=$ Most likely a serious problem - resolve or explain

0 ALERT level B = A potentially serious problem, consider carefully

12 ALERT level $\mathbf{C}=$ Check. Ensure it is not caused by an omission or oversight

50 ALERT level $\mathbf{G}=$ General information/check it is not something unexpected

2 ALERT type 1 CIF construction/syntax error, inconsistent or missing data

19 ALERT type 2 Indicator that the structure model may be wrong or deficient

7 ALERT type 3 Indicator that the structure quality may be low

34 ALERT type 4 Improvement, methodology, query or suggestion

0 ALERT type 5 Informative message, check 
It is advisable to attempt to resolve as many as possible of the alerts in all categories. Often the minor alerts point to easily fixed oversights, errors and omissions in your CIF or refinement strategy, so attention to these fine details can be worthwhile. In order to resolve some of the more serious problems it may be necessary to carry out additional measurements or structure refinements. However, the purpose of your study may justify the reported deviations and the more serious of these should normally be commented upon in the discussion or experimental section of a paper or in the "special_details" fields of the CIF. checkCIF was carefully designed to identify outliers and unusual parameters, but every test has its limitations and alerts that are not important in a particular case may appear. Conversely, the absence of alerts does not guarantee there are no aspects of the results needing attention. It is up to the individual to critically assess their own results and, if necessary, seek expert advice.

\section{Publication of your CIF in IUCr journals}

A basic structural check has been run on your CIF. These basic checks will be run on all CIFs submitted for publication in IUCr journals (Acta Crystallographica, Journal of Applied Crystallography, Journal of Synchrotron Radiation); however, if you intend to submit to Acta Crystallographica Section C or E or IUCrData, you should make sure that full publication checks are run on the final version of your CIF prior to submission.

\section{Publication of your CIF in other journals}

Please refer to the Notes for Authors of the relevant journal for any special instructions relating to CIF submission.

\section{PLATON version of 07/08/2019; check.def file version of 30/07/2019}


Datablock 8-heat3 - ellipsoid plot

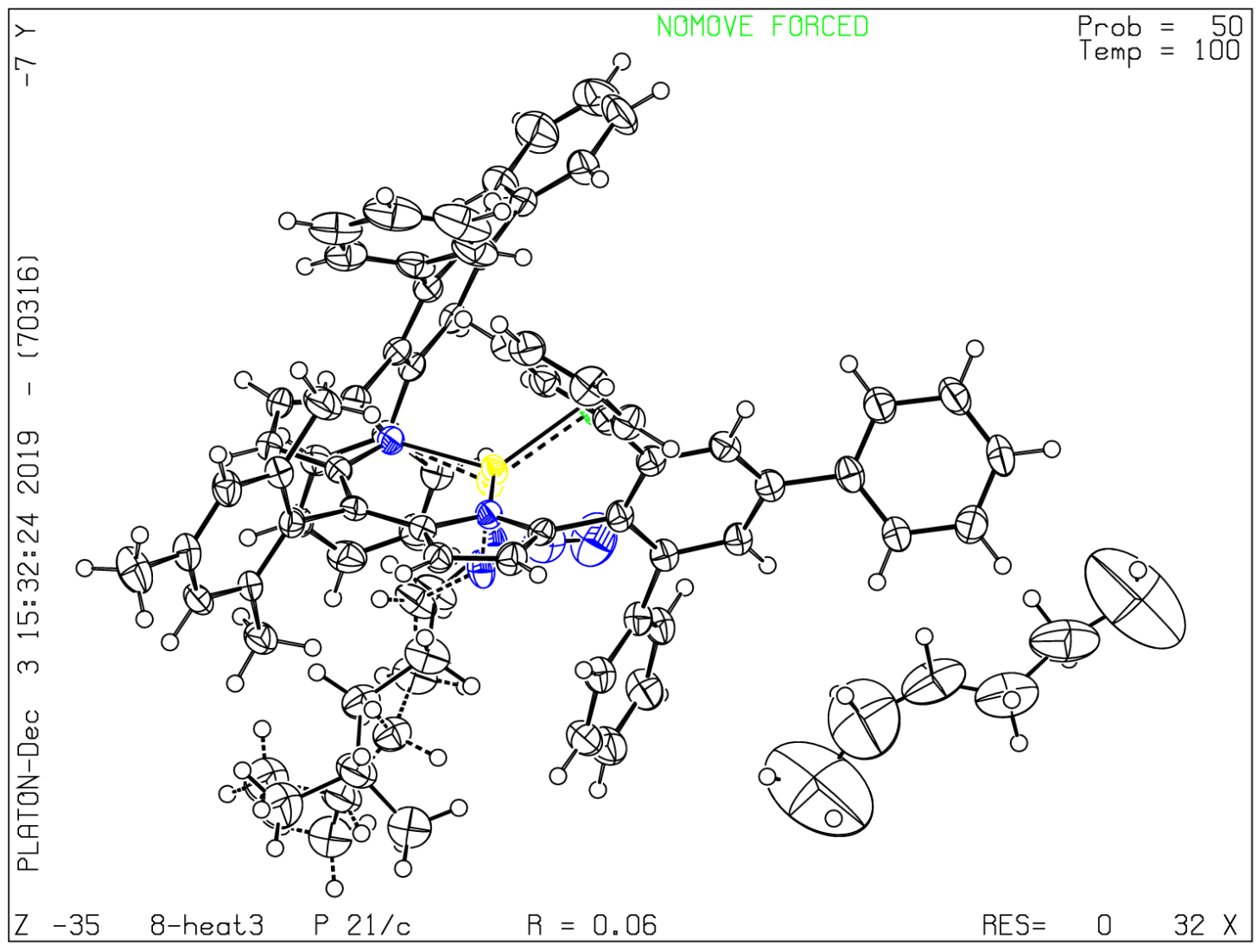




\section{checkCIF/PLATON report}

Structure factors have been supplied for datablock(s) 8-heat4

THIS REPORT IS FOR GUIDANCE ONLY. IF USED AS PART OF A REVIEW PROCEDURE FOR PUBLICATION, IT SHOULD NOT REPLACE THE EXPERTISE OF AN EXPERIENCED CRYSTALLOGRAPHIC REFEREE.

No syntax errors found. CIF dictionary Interpreting this report

\section{Datablock: 8-heat4}

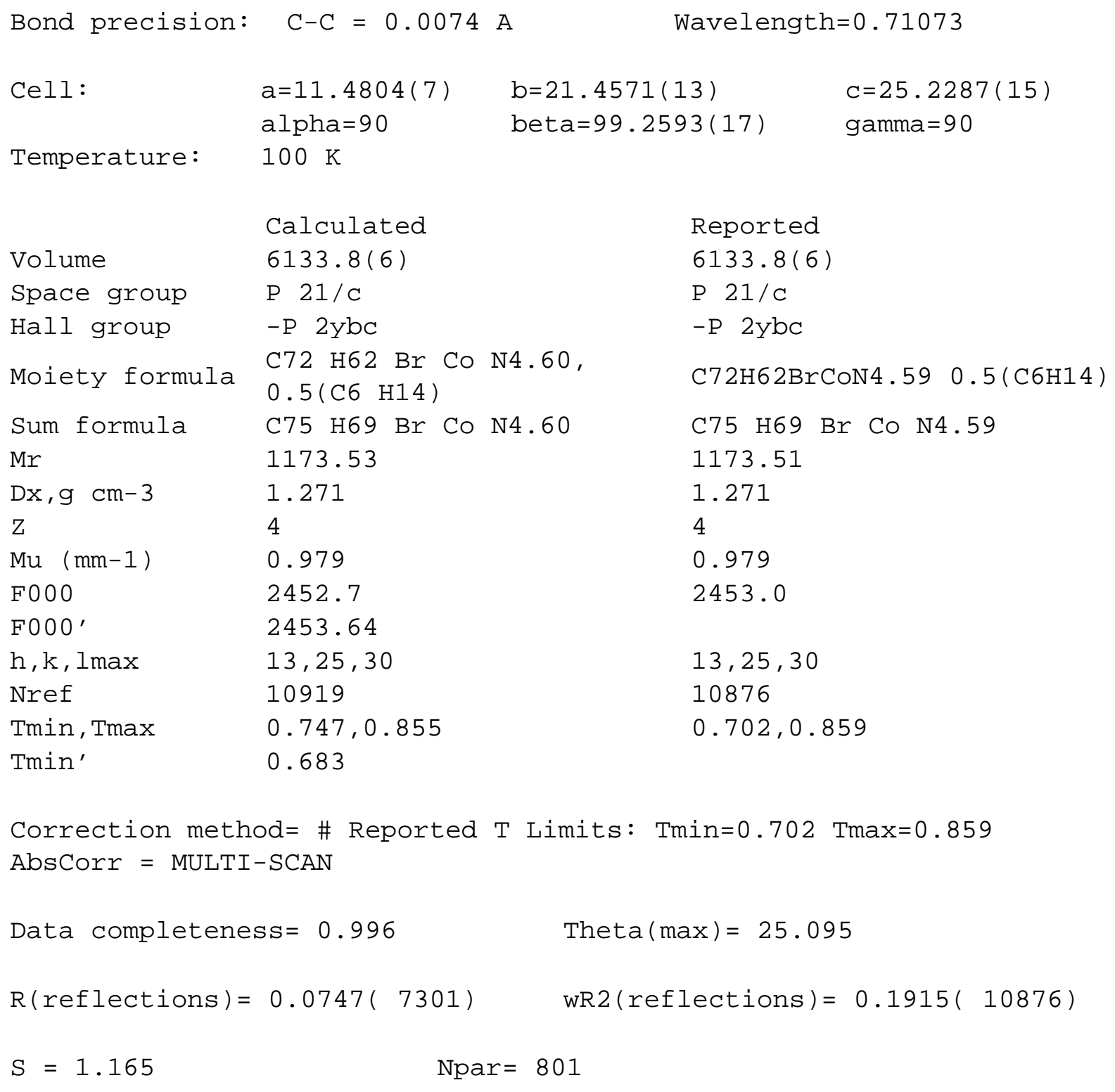


The following ALERTS were generated. Each ALERT has the format

test-name_ALERT_alert-type_alert-level.

Click on the hyperlinks for more details of the test.

Alert level C \begin{tabular}{|l|l}
\hline PLAT041_ALERT_1_C Calc. and Reported SumFormula Strings Differ \\
PLAT077_ALERT_4_C Unitcell Contains Non-integer Number of Atoms ..
\end{tabular} PLAT220_ALERT_2_C Non-Solvent Resd 1 C Ueq (max)/Ueq(min) Range PLAT220_ALERT_2_C Non-Solvent Resd $1 \mathrm{~N}$ Ueq (max)/Ueq(min) Range PLAT223_ALERT_4_C Solv./Anion Resd 2 H Ueq(max)/Ueq(min) Range PLAT234_ALERT_4_C Large Hirshfeld Difference C74 --C76 . PLAT250_ALERT_2_C Large U3/U1 Ratio for Average U(i,j) Tensor .... PLAT260_ALERT_2_C Large Average Ueq of Residue Including C1S

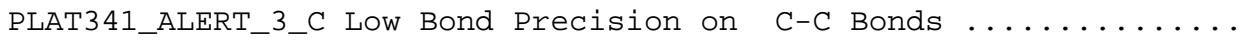
PLAT411_ALERT_2_C Short Inter H...H Contact H17 ..H17 .

PLAT906_ALERT_3_C Large $K$ Value in the Analysis of Variance ...... PLAT906_ALERT_3_C Large $\mathrm{K}$ Value in the Analysis of Variance ...... PLAT911_ALERT_3_C Missing FCF Refl Between Thmin \& STh/L= 0.597 PLAT977_ALERT_2_C Check Negative Difference Density on H71C PLAT978_ALERT_2_C Number C-C Bonds with Positive Residual Density.

\author{
Please Check \\ Please Check \\ 3.8 Ratio \\ 3. 4 Ratio \\ 4.3 Ratio \\ 0.19 Ang. \\ 3.7 Note \\ 0.188 Check \\ 0.00742 Ang. \\ 2.10 Ang. \\ 3_666 Check \\ 7.008 Check \\ 2.295 Check \\ 41 Report \\ -0.32 eA-3 \\ 0 Info
}

\section{Alert level G}

FORMU01_ALERT_1_G There is a discrepancy between the atom counts in the _chemical_formula_sum and_chemical_formula_moiety. This is usually due to the moiety formula being in the wrong format. Atom count from_chemical_formula_sum: C75 H69 Br1 Co1 N4.59 Atom count from_chemical_formula_moiety:C78 H76 Br1 Co1 N40590.50

PLAT002_ALERT_2_G Number of Distance or Angle Restraints on AtSite PLAT003_ALERT_2_G Number of Uiso or Uij Restrained non-H Atoms ... PLAT042_ALERT_1_G Calc. and Reported MoietyFormula Strings Differ PLAT068_ALERT_1_G Reported F000 Differs from Calcd (or Missing)... PLAT083_ALERT_2_G SHELXL Second Parameter in WGHT Unusually Large PLAT171_ALERT_4_G The CIF-Embedded.res File Contains EADP Records PLAT172_ALERT_4_G The CIF-Embedded.res File Contains DFIX Records PLAT175_ALERT_4_G The CIF-Embedded .res File Contains SAME Records PLAT176_ALERT_4_G The CIF-Embedded.res File Contains SADI Records PLAT178_ALERT_4_G The CIF-Embedded.res File Contains SIMU Records PLAT186_ALERT_4_G The CIF-Embedded.res File Contains ISOR Records PLAT187_ALERT_4_G The CIF-Embedded.res File Contains RIGU Records PLAT230_ALERT_2_G Hirshfeld Test Diff for N3 PLAT300_ALERT_4_G Atom Site Occupancy of C1S PLAT300_ALERT_4_G Atom Site Occupancy of C2S PLAT300_ALERT_4_G Atom Site Occupancy of C3S PLAT300_ALERT_4_G Atom Site Occupancy of C4S PLAT300_ALERT_4_G Atom Site Occupancy of C5S PLAT300_ALERT_4_G Atom Site Occupancy of C6S PLAT300_ALERT_4_G Atom Site Occupancy of H1SA PLAT300_ALERT_4_G Atom Site Occupancy of H1SB PLAT300_ALERT_4_G Atom Site Occupancy of H1SC PLAT300_ALERT_4_G Atom Site Occupancy of H2SA PLAT300_ALERT_4_G Atom Site Occupancy of H2SB PLAT300_ALERT_4_G Atom Site Occupancy of H3SA PLAT300_ALERT_4_G Atom Site Occupancy of H3SB PLAT300_ALERT_4_G Atom Site Occupancy of H4SA PLAT300_ALERT_4_G Atom Site Occupancy of H4SB PLAT300_ALERT_4_G Atom Site Occupancy of H5SA PLAT300_ALERT_4_G Atom Site Occupancy of H5SB PLAT300_ALERT_4_G Atom Site Occupancy of H6SA
Constrained at Constrained at Constrained at Constrained at Constrained at Constrained at Constrained at Constrained at Constrained at Constrained at Constrained at Constrained at Constrained at Constrained at Constrained at Constrained at Constrained at Constrained at
20 Note

6 Report

Please Check Please Check 11.75 Why ?

9 Report

1 Report

2 Report

2 Report

1 Report

1 Report

1 Report

12.8 s.u.

0.5 Check

0.5 Check

0.5 Check

0.5 Check

0.5 Check

0.5 Check

0.5 Check

0.5 Check

0.5 Check

0.5 Check

0.5 Check

0.5 Check

0.5 Check

0.5 Check

0.5 Check

0.5 Check

0.5 Check

0.5 Check 


\begin{tabular}{|c|c|c|c|c|c|}
\hline PLAT300_ALERT_4_G & Atom Site Occupancy of $\mathrm{H} 6 \mathrm{~S}$ & Constrair & ned & at & 0.5 Check \\
\hline PLAT300_ALERT_4_G & Atom Site Occupancy of $\mathrm{H} 6 \mathrm{~S}$ & Constrair & ned & at & 0.5 Check \\
\hline PLAT301_ALERT_3_G & Main Residue Disorder ... & $\ldots \ldots \ldots(\operatorname{Res} d$ & 1 & ) & $13 \%$ Note \\
\hline PLAT302_ALERT_4_G & Anion/Solvent/Minor-Residu & ue Disorder (Resd & 2 & ) & $100 \%$ Note \\
\hline PLAT304_ALERT_4_G & Non-Integer Number of Atom & $m s$ in $\ldots . . \operatorname{Resd}$ & 1 & & 140.60 Check \\
\hline PLAT 411_ALERT_2_G & hort Inter $\mathrm{H} . . . \mathrm{H}$ Contact & $\begin{array}{cc}\mathrm{H} 2 \mathrm{SB} & \ldots \mathrm{H} 28 \\
1-\mathrm{x},-1 / 2+\mathrm{y}, 3 / 2-\mathrm{z}\end{array}$ & $=$ & . & $\begin{array}{l}2.04 \text { Ang. } \\
\text { 2_646 Check }\end{array}$ \\
\hline SLAT413_ALE & rt Inter XH3 .. XHn & $\begin{array}{rr}\text { H14 } & \cdots \text { H75D } \\
& -x, 1-y, 1-z\end{array}$ & $=$ & . & $\begin{array}{l}1.75 \text { Ang. } \\
\text { 3_566 Check }\end{array}$ \\
\hline PLAT413_ALERT_2_G & rt Inter XH3 .. XHn & $\begin{array}{cc}\text { H4 } 4 & \cdots \mathrm{H} 75 \mathrm{~F} \\
1+\mathrm{x}, 1 / 2-\mathrm{y}, 1 / 2+\mathrm{z}\end{array}$ & $=$ & • & $\begin{array}{l}1.83 \text { Ang. } \\
4 \_666 \text { Check }\end{array}$ \\
\hline PLAT 413_ALERT_2_G & hort Inter $\mathrm{XH} 3$ & $\begin{array}{lr}\mathrm{H} 1 \mathrm{SB} & \mathrm{HH} 27 \\
\mathrm{x}, 1 / 2-\mathrm{y},-1 / 2+\mathrm{z}\end{array}$ & & . & $\begin{array}{l}1.57 \text { Ang. } \\
4 \_565 \text { Check }\end{array}$ \\
\hline LAT432_ALERT_2 & rt Inter X...Y Cor & $\begin{array}{c}\text { C13 } \\
-x, 1-y, 1-z\end{array}$ & $=$ & & $\begin{array}{l}2.85 \text { Ang. } \\
\text { 3_566 Check }\end{array}$ \\
\hline PLAT4 32_ALERT_2_G & hort Inter X...Y Contact & $\begin{array}{r}\cdots C 75 B \\
-x, 1-y, 1-z\end{array}$ & $=$ & & $\begin{array}{l}2.47 \text { Ang. } \\
\text { 3_566 Check }\end{array}$ \\
\hline PLAT 4 32_ALERT_2_G & Short Inter & $\begin{array}{c}\text { C15 } \\
\\
-x, 1-y, 1-z\end{array}$ & $=$ & & $\begin{array}{l}3.08 \text { Ang. } \\
\text { 3_566 Check }\end{array}$ \\
\hline PLAT4 32_ALERT_2_G & Inter X...Y & $\begin{array}{c}\text { C44 } \quad \text {. C75B } \\
1+x, 1 / 2-y, 1 / 2+z\end{array}$ & $=$ & & $\begin{array}{l}3.01 \text { Ang. } \\
4 \_666 \text { Check }\end{array}$ \\
\hline PLAT720_ALERT_4_G & Iumber of Unusual/Non-Stan & ndard Labels .... & & & 14 Note \\
\hline PLAT789_ALERT_4_G & Atoms with Negative _atom & _site_disorder_gro & oup & \# & $20 \mathrm{Ch}$ \\
\hline PLAT860_ALERT_3_G & Number of Least-Squares $R e$ & estraints ...... & $\cdots \cdot$ & & 115 No \\
\hline PLAT909_ALERT_3_G & Percentage of I $>2$ sig(I) Da & ata at Theta (Max) & Sti & ill & $39 \%$ Note \\
\hline PLAT910_ALERT_3_G & Missing \# of FCF Reflectio & on (s) Below Theta & & & 3 \\
\hline PLAT933_ALERT_2_G & Number of OMIT Records in & Embedded .res Fil & & & 6 Note \\
\hline
\end{tabular}

0 ALERT level $\mathbf{A}=$ Most likely a serious problem - resolve or explain

0 ALERT level $\mathbf{B}=\mathrm{A}$ potentially serious problem, consider carefully

15 ALERT level $\mathbf{C}=$ Check. Ensure it is not caused by an omission or oversight

51 ALERT level $\mathbf{G}=$ General information/check it is not something unexpected

4 ALERT type 1 CIF construction/syntax error, inconsistent or missing data

20 ALERT type 2 Indicator that the structure model may be wrong or deficient

8 ALERT type 3 Indicator that the structure quality may be low

34 ALERT type 4 Improvement, methodology, query or suggestion

0 ALERT type 5 Informative message, check 
It is advisable to attempt to resolve as many as possible of the alerts in all categories. Often the minor alerts point to easily fixed oversights, errors and omissions in your CIF or refinement strategy, so attention to these fine details can be worthwhile. In order to resolve some of the more serious problems it may be necessary to carry out additional measurements or structure refinements. However, the purpose of your study may justify the reported deviations and the more serious of these should normally be commented upon in the discussion or experimental section of a paper or in the "special_details" fields of the CIF. checkCIF was carefully designed to identify outliers and unusual parameters, but every test has its limitations and alerts that are not important in a particular case may appear. Conversely, the absence of alerts does not guarantee there are no aspects of the results needing attention. It is up to the individual to critically assess their own results and, if necessary, seek expert advice.

\section{Publication of your CIF in IUCr journals}

A basic structural check has been run on your CIF. These basic checks will be run on all CIFs submitted for publication in IUCr journals (Acta Crystallographica, Journal of Applied Crystallography, Journal of Synchrotron Radiation); however, if you intend to submit to Acta Crystallographica Section C or E or IUCrData, you should make sure that full publication checks are run on the final version of your CIF prior to submission.

\section{Publication of your CIF in other journals}

Please refer to the Notes for Authors of the relevant journal for any special instructions relating to CIF submission.

\section{PLATON version of 07/08/2019; check.def file version of 30/07/2019}


Datablock 8-heat4 - ellipsoid plot

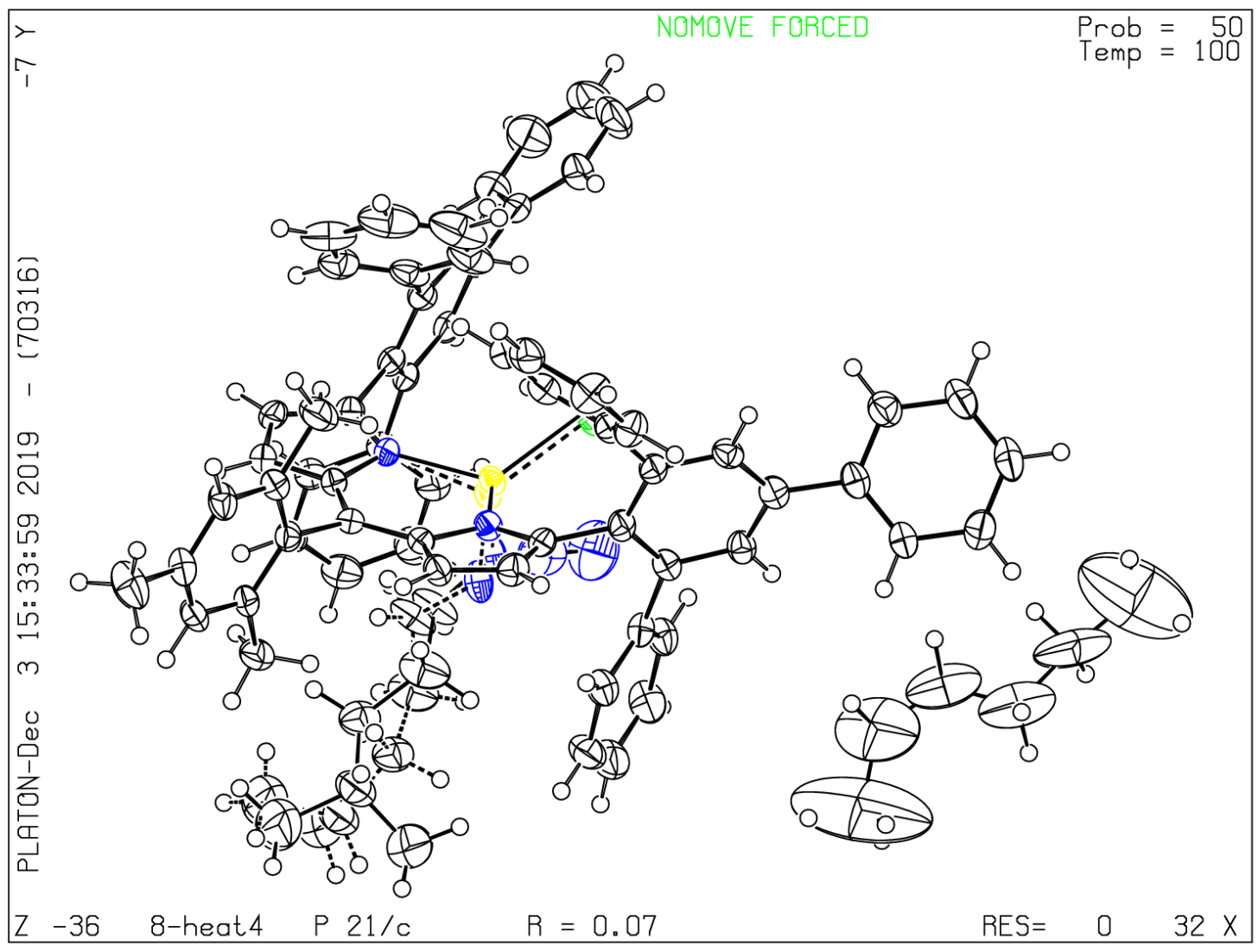




\section{checkCIF/PLATON report}

You have not supplied any structure factors. As a result the full set of tests cannot be run.

THIS REPORT IS FOR GUIDANCE ONLY. IF USED AS PART OF A REVIEW PROCEDURE FOR PUBLICATION, IT SHOULD NOT REPLACE THE EXPERTISE OF AN EXPERIENCED CRYSTALLOGRAPHIC REFEREE.

No syntax errors found. CIF dictionary Interpreting this report

\section{Datablock: 7_start}

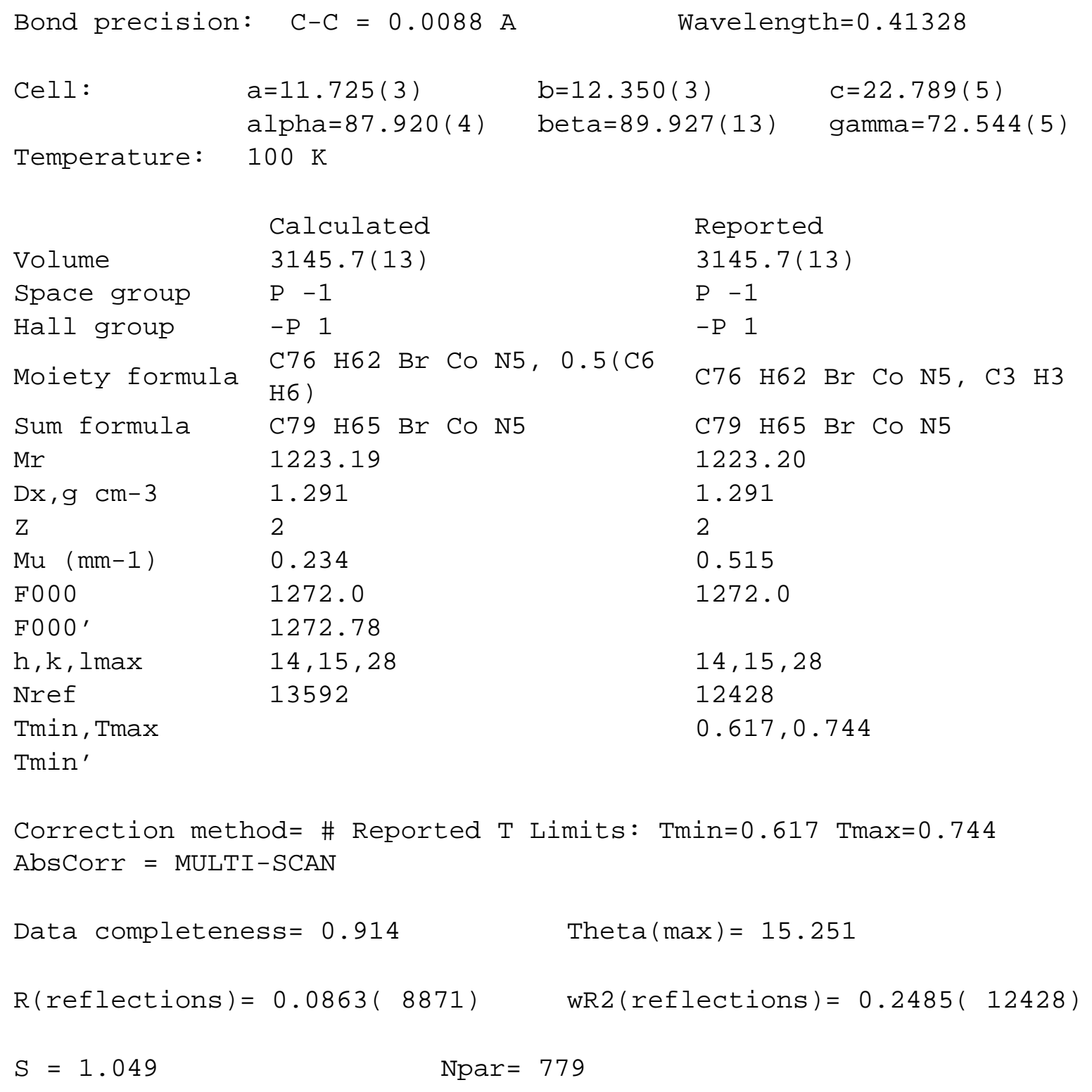


The following ALERTS were generated. Each ALERT has the format

test-name_ALERT_alert-type_alert-level.

Click on the hyperlinks for more details of the test.

Alert level $\mathrm{A}$

PLAT029_ALERT_3_A__iffrn_measured_fraction_theta_full value Low. 0.939 Why?

Author Response: Data was collected at a synchrotron source as a series of phi scans and this is the completeness that was obtained by this acquisition sequence. The geometry of the experiment (cryostat, LED fiber optic, detector, and beam path) prevented acquisition of omega scans.

Alert level $\mathrm{C}$

RINTA01_ALERT_3_C The value of Rint is greater than 0.12

$$
\text { Rint given } 0.138
$$

PLAT020_ALERT_3_C The Value of Rint is Greater Than $0.12 \ldots \ldots . . .0 .138$ Report

PLAT341_ALERT_3_C Low Bond Precision on C-C Bonds ............ 0.0088 Ang.

Alert level $\mathbf{G}$

ABSMU01_ALERT_1_G Calculation of _expt1_absorpt_correction_mu

not performed for this radiation type.

PLAT042_ALERT_1_G Calc. and Reported MoietyFormula Strings Differ

PLAT083_ALERT_2_G SHELXL Second Parameter in WGHT Unusually Large

PLAT092_ALERT_4_G Check: Wavelength Given is not Cu, Ga, Mo, Ag, In Ka

PLAT720_ALERT_4_G Number of Unusual/Non-Standard Labels ........

PLAT883_ALERT_1_G No Info/Value for_atom_sites_solution_primary .

PLAT984_ALERT_1_G The $B r-f^{\prime}=0.2880$ Deviates from the B\&C-Value

PLAT985_ALERT_1_G The Br-f"= 0.9627 Deviates from the B\&C-Value

PLAT985_ALERT_1_G The Co-f" $=0.3155$ Deviates from the B\&C-Value

$$
\begin{array}{r}
\text { Please Check } \\
14.35 \text { Why ? } \\
0.41328 \text { Ang. } \\
3 \text { Note } \\
\text { Please Do ! } \\
0.2863 \text { Check } \\
0.9639 \text { Check } \\
0.3559 \text { Check }
\end{array}
$$

1 ALERT level $\mathbf{A}=$ Most likely a serious problem - resolve or explain

0 ALERT level $\mathbf{B}=\mathrm{A}$ potentially serious problem, consider carefully

3 ALERT level $\mathbf{C}=$ Check. Ensure it is not caused by an omission or oversight

9 ALERT level $\mathbf{G}=$ General information/check it is not something unexpected

6 ALERT type 1 CIF construction/syntax error, inconsistent or missing data

1 ALERT type 2 Indicator that the structure model may be wrong or deficient

4 ALERT type 3 Indicator that the structure quality may be low

2 ALERT type 4 Improvement, methodology, query or suggestion

0 ALERT type 5 Informative message, check 
It is advisable to attempt to resolve as many as possible of the alerts in all categories. Often the minor alerts point to easily fixed oversights, errors and omissions in your CIF or refinement strategy, so attention to these fine details can be worthwhile. In order to resolve some of the more serious problems it may be necessary to carry out additional measurements or structure refinements. However, the purpose of your study may justify the reported deviations and the more serious of these should normally be commented upon in the discussion or experimental section of a paper or in the "special_details" fields of the CIF. checkCIF was carefully designed to identify outliers and unusual parameters, but every test has its limitations and alerts that are not important in a particular case may appear. Conversely, the absence of alerts does not guarantee there are no aspects of the results needing attention. It is up to the individual to critically assess their own results and, if necessary, seek expert advice.

\section{Publication of your CIF in IUCr journals}

A basic structural check has been run on your CIF. These basic checks will be run on all CIFs submitted for publication in IUCr journals (Acta Crystallographica, Journal of Applied Crystallography, Journal of Synchrotron Radiation); however, if you intend to submit to Acta Crystallographica Section C or E or IUCrData, you should make sure that full publication checks are run on the final version of your CIF prior to submission.

\section{Publication of your CIF in other journals}

Please refer to the Notes for Authors of the relevant journal for any special instructions relating to CIF submission.

\section{PLATON version of 22/04/2020; check.def file version of 09/03/2020}


Datablock 7_start - ellipsoid plot

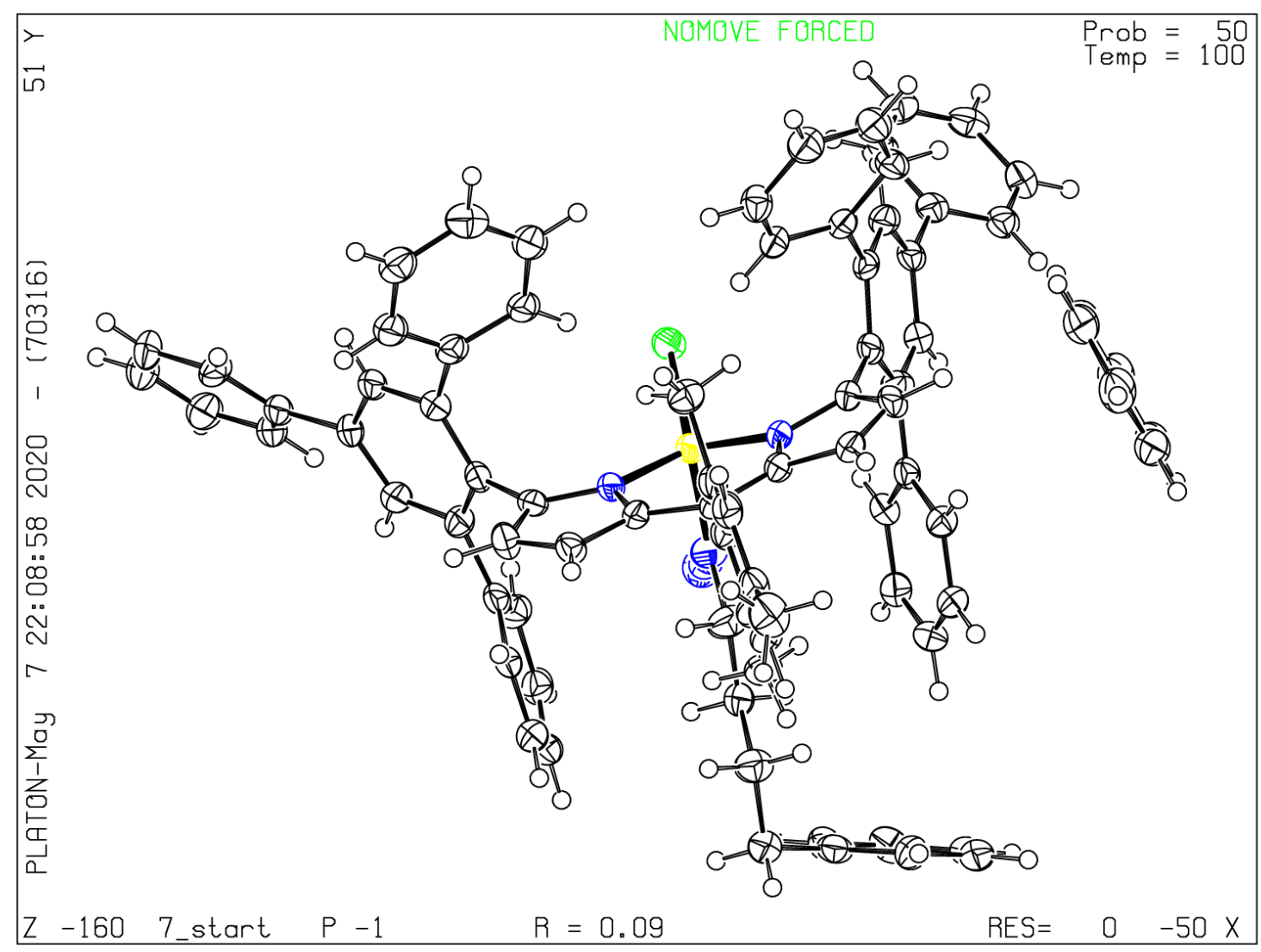




\section{checkCIF/PLATON report}

You have not supplied any structure factors. As a result the full set of tests cannot be run.

THIS REPORT IS FOR GUIDANCE ONLY. IF USED AS PART OF A REVIEW PROCEDURE FOR PUBLICATION, IT SHOULD NOT REPLACE THE EXPERTISE OF AN EXPERIENCED CRYSTALLOGRAPHIC REFEREE.

No syntax errors found. CIF dictionary Interpreting this report

\section{Datablock: 7-N2}

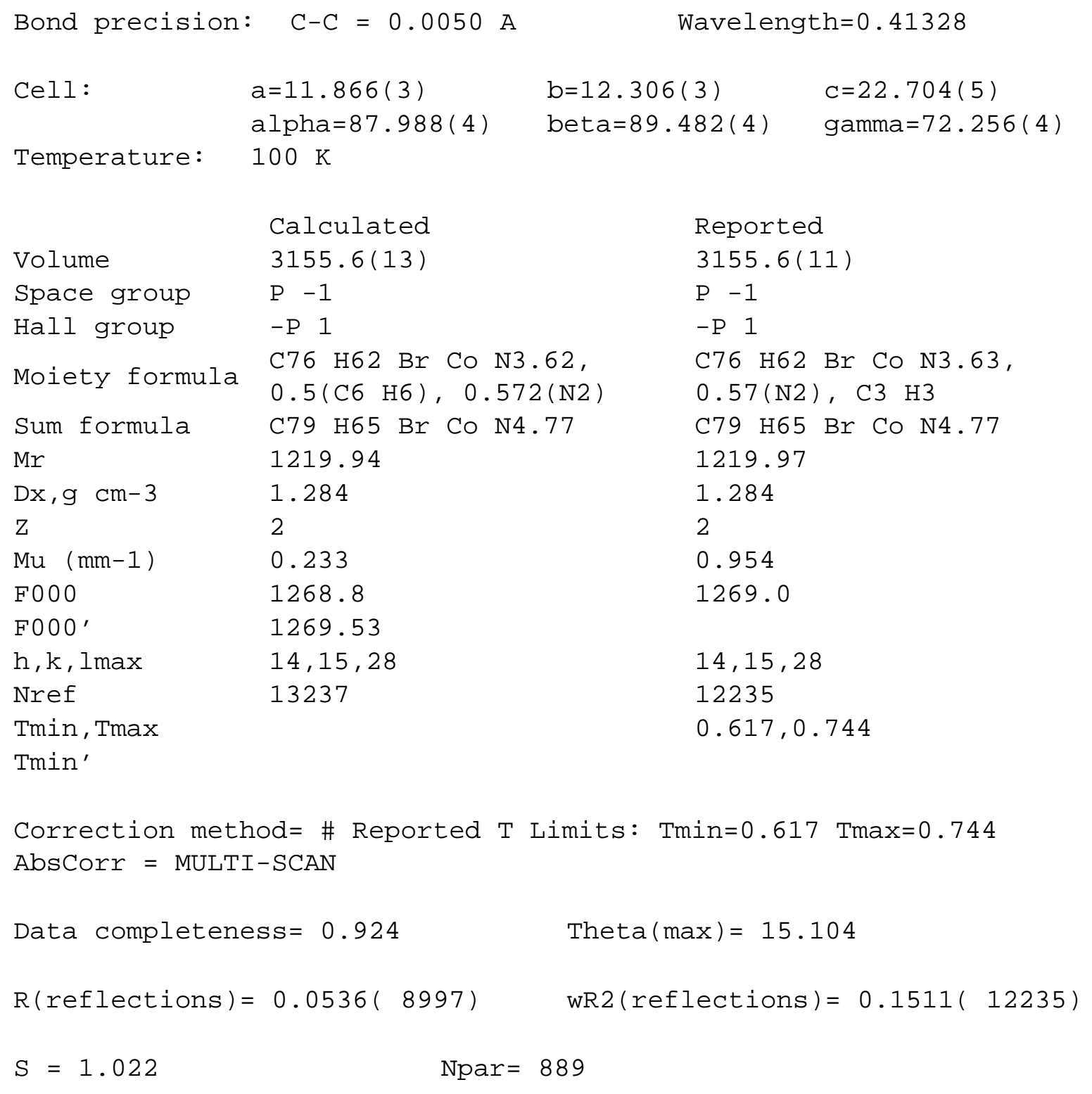


The following ALERTS were generated. Each ALERT has the format

test-name_ALERT_alert-type_alert-level.

Click on the hyperlinks for more details of the test.

Alert level A

PLAT029_ALERT_3_A _diffrn_measured_fraction_theta_full value Low . 0.936 Why?

\begin{abstract}
Author Response: Data was collected at a synchrotron source as a series of phi scans and this is the completeness that was obtained by this acquisition sequence. The geometry of the experiment (cryostat, LED fiber optic, detector, and beam path) prevented acquisition of omega scans.
\end{abstract}

Alert level $\mathrm{C}$

PLAT077_ALERT_4_C Unitcell Contains Non-integer Number of Atoms .. PLAT220_ALERT_2_C NonSolvent Resd 1 C Ueq(max) / Ueq(min) Range PLAT234_ALERT_4_C Large Hirshfeld Difference C1_2 --C2_2 .

Please Check

3.2 Ratio

0.16 Ang.

Alert level $\mathbf{G}$

ABSMU01_ALERT_1_G Calculation of _expt1_absorpt_correction_mu not performed for this radiation type.

PLAT002_ALERT_2_G Number of Distance or Angle Restraints on AtSite PLAT003_ALERT_2_G Number of Uiso or Uij Restrained non-H Atoms ... PLAT042_ALERT_1_G Calc. and Reported MoietyFormula Strings Differ PLAT092_ALERT_4_G Check: Wavelength Given is not $\mathrm{Cu}, \mathrm{Ga}, \mathrm{Mo}, \mathrm{Ag}, \mathrm{In} \mathrm{Ka}$ PLAT152_ALERT_1_G The Supplied and Calc. Volume s.u. Differ by ... PLAT154_ALERT_1_G The s.u.'s on the Cell Angles are Equal .. (Note) PLAT172_ALERT_4_G The CIF-Embedded.res File Contains DFIX Records PLAT175_ALERT_4_G The CIF-Embedded.res File Contains SAME Records PLAT176_ALERT_4_G The CIF-Embedded .res File Contains SADI Records PLAT177_ALERT_4_G The CIF-Embedded.res File Contains DELU Records PLAT178_ALERT_4_G The CIF-Embedded.res File Contains SIMU Records PLAT230_ALERT_2_G Hirshfeld Test Diff for N1A --C1_2 . PLAT232_ALERT_2_G Hirshfeld Test Diff (M-X) Br1 --Co1. PLAT301_ALERT_3_G Main Residue Disorder...............Resd 1 ) PLAT302_ALERT_4_G Anion/Solvent/Minor-Residue Disorder (Resd 3 ) PLAT304_ALERT_4_G Non-Integer Number of Atoms in ..... (Resd 1 ) PLAT304_ALERT_4_G Non-Integer Number of Atoms in ..... (Resd 3 ) PLAT720_ALERT_4_G Number of Unusual/Non-Standard Labels ........

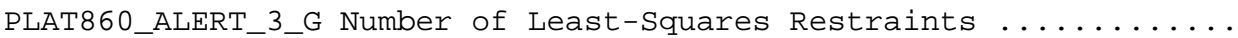
PLAT984_ALERT_1_G The $B r-f^{\prime}=0.2880$ Deviates from the B\&C-Value PLAT985_ALERT_1_G The Br-f"= 0.9627 Deviates from the B\&C-Value PLAT985_ALERT_1_G The Co-f"= 0.3155 Deviates from the B\&C-Value

$$
\begin{aligned}
23 & \text { Note } \\
23 & \text { Report } \\
\text { Please } & \text { Check } \\
0.41328 & \text { Ang. } \\
2 & \text { Units } \\
0.004 & \text { Degree } \\
3 & \text { Report } \\
1 & \text { Report } \\
1 & \text { Report } \\
3 & \text { Report } \\
3 & \text { Report } \\
5.2 & \text { s.u. } \\
10.3 & \text { s.u. } \\
13 \% & \text { Note } \\
100 \% & \text { Note } \\
143.62 & \text { Check } \\
1.14 & \text { Check } \\
42 & \text { Note } \\
174 & \text { Note } \\
0.2863 & \text { Check } \\
0.9639 & \text { Check } \\
0.3559 & \text { Check }
\end{aligned}
$$

\footnotetext{
1 ALERT level $\mathbf{A}=$ Most likely a serious problem - resolve or explain

0 ALERT level $\mathbf{B}=\mathrm{A}$ potentially serious problem, consider carefully

3 ALERT level $\mathbf{C}=$ Check. Ensure it is not caused by an omission or oversight

23 ALERT level $\mathbf{G}=$ General information/check it is not something unexpected

7 ALERT type 1 CIF construction/syntax error, inconsistent or missing data

5 ALERT type 2 Indicator that the structure model may be wrong or deficient

3 ALERT type 3 Indicator that the structure quality may be low

12 ALERT type 4 Improvement, methodology, query or suggestion

0 ALERT type 5 Informative message, check
} 
It is advisable to attempt to resolve as many as possible of the alerts in all categories. Often the minor alerts point to easily fixed oversights, errors and omissions in your CIF or refinement strategy, so attention to these fine details can be worthwhile. In order to resolve some of the more serious problems it may be necessary to carry out additional measurements or structure refinements. However, the purpose of your study may justify the reported deviations and the more serious of these should normally be commented upon in the discussion or experimental section of a paper or in the "special_details" fields of the CIF. checkCIF was carefully designed to identify outliers and unusual parameters, but every test has its limitations and alerts that are not important in a particular case may appear. Conversely, the absence of alerts does not guarantee there are no aspects of the results needing attention. It is up to the individual to critically assess their own results and, if necessary, seek expert advice.

\section{Publication of your CIF in IUCr journals}

A basic structural check has been run on your CIF. These basic checks will be run on all CIFs submitted for publication in IUCr journals (Acta Crystallographica, Journal of Applied Crystallography, Journal of Synchrotron Radiation); however, if you intend to submit to Acta Crystallographica Section C or E or IUCrData, you should make sure that full publication checks are run on the final version of your CIF prior to submission.

\section{Publication of your CIF in other journals}

Please refer to the Notes for Authors of the relevant journal for any special instructions relating to CIF submission.

\section{PLATON version of 22/04/2020; check.def file version of $09 / 03 / 2020$}




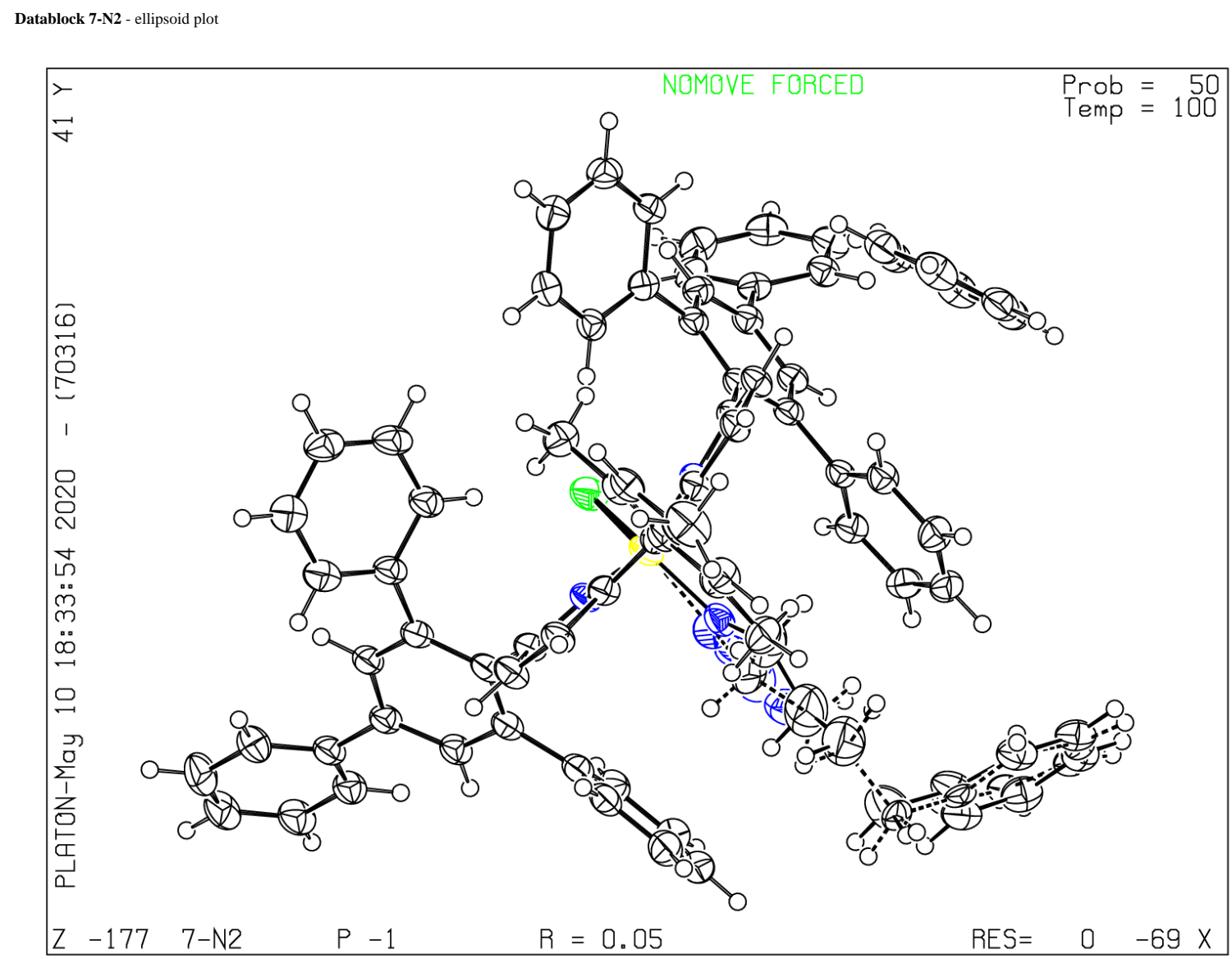




\section{checkCIF/PLATON report}

You have not supplied any structure factors. As a result the full set of tests cannot be run.

THIS REPORT IS FOR GUIDANCE ONLY. IF USED AS PART OF A REVIEW PROCEDURE FOR PUBLICATION, IT SHOULD NOT REPLACE THE EXPERTISE OF AN EXPERIENCED CRYSTALLOGRAPHIC REFEREE.

No syntax errors found. CIF dictionary Interpreting this report

\section{Datablock: 8_start}

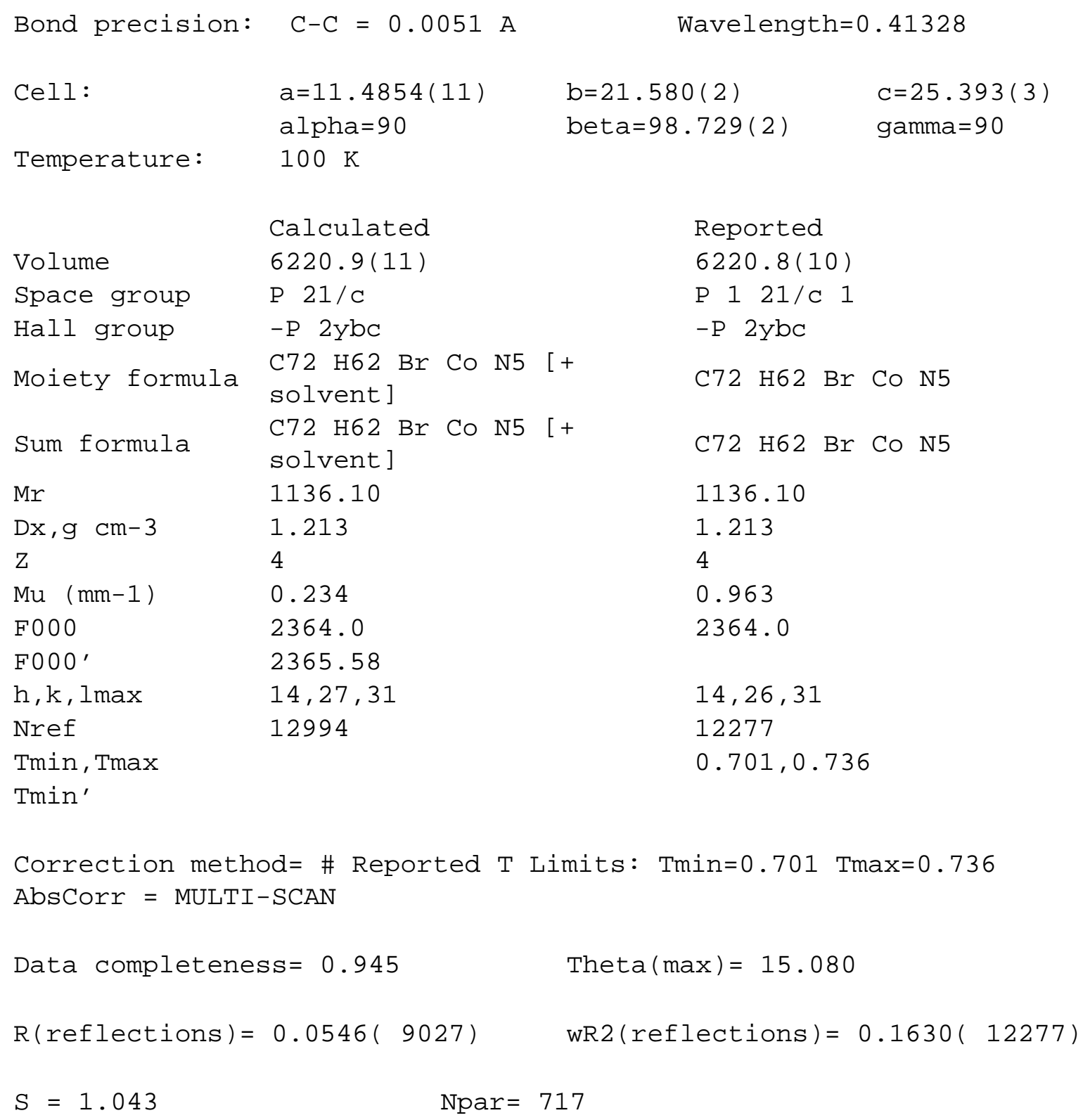


The following ALERTS were generated. Each ALERT has the format

test-name_ALERT_alert-type_alert-level.

Click on the hyperlinks for more details of the test.

Alert level $B$

PLAT029_ALERT_3_B _diffrn_measured_fraction_theta_full value Low . 0.953 Why?

Author Response: Data was collected at a synchrotron source as a series of phi scans and this is the completeness that was obtained by this acquisition sequence. The geometry of the experiment (cryostat, LED fiber optic, detector, and beam path) prevented acquisition of omega scans.

Alert level C

PLAT220_ALERT_2_C NonSolvent Resd 1 C Ueq(max) / Ueq(min) Range PLAT222_ALERT_3_C NonSolvent Resd 1 H Uiso(max)/Uiso(min) Range PLAT242_ALERT_2_C Low 'MainMol' Ueq as Compared to Neighbors of PLAT242_ALERT_2_C Low 'MainMol' Ueq as Compared to Neighbors of PLAT360_ALERT_2_C Short C (sp3)-C(sp3) Bond C4 - C6 . PLAT411_ALERT_2_C Short Inter H...H Contact H68
6 .0 Ratio

7.0 Ratio

C3 Check

C4 Check

1.38 Ang.

2.13 Ang.

3_666 Check

Alert level $\mathbf{G}$

ABSMU01_ALERT_1_G Calculation of _expt1_absorpt_correction_mu not performed for this radiation type.

PLAT002_ALERT_2_G Number of Distance or Angle Restraints on AtSite PLAT003_ALERT_2_G Number of Uiso or Uij Restrained non-H Atoms ... PLAT092_ALERT_4_G Check: Wavelength Given is not $\mathrm{Cu}, \mathrm{Ga}, \mathrm{Mo}, \mathrm{Ag}$, In Ka PLAT172_ALERT_4_G The CIF-Embedded.res File Contains DFIX Records PLAT176_ALERT_4_G The CIF-Embedded.res File Contains SADI Records PLAT187_ALERT_4_G The CIF-Embedded .res File Contains RIGU Records PLAT606_ALERT_4_G VERY LARGE Solvent Accessible VOID(S) in structure PLAT860_ALERT_3_G Number of Least-Squares Restraints ........... PLAT883_ALERT_1_G No Info/Value for_atom_sites_solution_primary . PLAT933_ALERT_2_G Number of OMIT Records in Embedded.res File... PLAT984_ALERT_1_G The Br-f'= 0.2880 Deviates from the B\&C-Value PLAT985_ALERT_1_G The Br-f"= 0.9627 Deviates from the B\&C-Value PLAT985_ALERT_1_G The Co-f"= 0.3155 Deviates from the B\&C-Value

$$
\begin{aligned}
10 & \text { Note } \\
6 & \text { Report } \\
0.41328 & \text { Ang. } \\
5 & \text { Report } \\
1 & \text { Report } \\
1 & \text { Report } \\
! & \text { Info } \\
44 & \text { Note } \\
\text { Please } & \text { Do ! } \\
1 & \text { Note } \\
0.2863 & \text { Check } \\
0.9639 & \text { Check } \\
0.3559 & \text { Check }
\end{aligned}
$$

0 ALERT level $\mathbf{A}=$ Most likely a serious problem - resolve or explain

1 ALERT level B = A potentially serious problem, consider carefully

6 ALERT level $\mathbf{C}=$ Check. Ensure it is not caused by an omission or oversight

14 ALERT level $\mathbf{G}=$ General information/check it is not something unexpected

5 ALERT type 1 CIF construction/syntax error, inconsistent or missing data

8 ALERT type 2 Indicator that the structure model may be wrong or deficient

3 ALERT type 3 Indicator that the structure quality may be low

5 ALERT type 4 Improvement, methodology, query or suggestion

0 ALERT type 5 Informative message, check 
It is advisable to attempt to resolve as many as possible of the alerts in all categories. Often the minor alerts point to easily fixed oversights, errors and omissions in your CIF or refinement strategy, so attention to these fine details can be worthwhile. In order to resolve some of the more serious problems it may be necessary to carry out additional measurements or structure refinements. However, the purpose of your study may justify the reported deviations and the more serious of these should normally be commented upon in the discussion or experimental section of a paper or in the "special_details" fields of the CIF. checkCIF was carefully designed to identify outliers and unusual parameters, but every test has its limitations and alerts that are not important in a particular case may appear. Conversely, the absence of alerts does not guarantee there are no aspects of the results needing attention. It is up to the individual to critically assess their own results and, if necessary, seek expert advice.

\section{Publication of your CIF in IUCr journals}

A basic structural check has been run on your CIF. These basic checks will be run on all CIFs submitted for publication in IUCr journals (Acta Crystallographica, Journal of Applied Crystallography, Journal of Synchrotron Radiation); however, if you intend to submit to Acta Crystallographica Section C or E or IUCrData, you should make sure that full publication checks are run on the final version of your CIF prior to submission.

\section{Publication of your CIF in other journals}

Please refer to the Notes for Authors of the relevant journal for any special instructions relating to CIF submission.

\section{PLATON version of 22/04/2020; check.def file version of 09/03/2020}


Datablock 8_start - ellipsoid plot

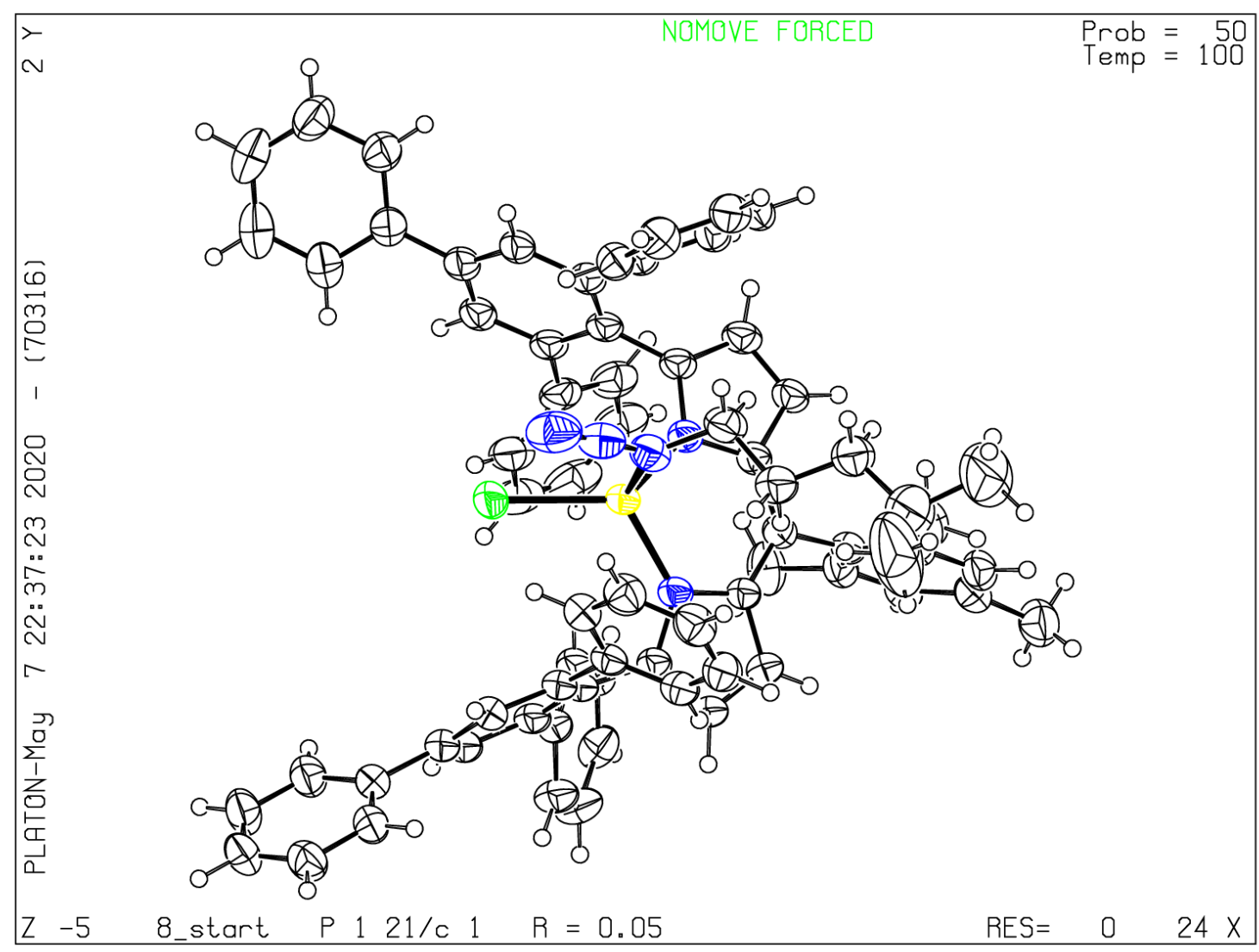




\section{checkCIF/PLATON report}

You have not supplied any structure factors. As a result the full set of tests cannot be run.

THIS REPORT IS FOR GUIDANCE ONLY. IF USED AS PART OF A REVIEW PROCEDURE FOR PUBLICATION, IT SHOULD NOT REPLACE THE EXPERTISE OF AN EXPERIENCED CRYSTALLOGRAPHIC REFEREE.

No syntax errors found. CIF dictionary Interpreting this report

\section{Datablock: 8-N2}

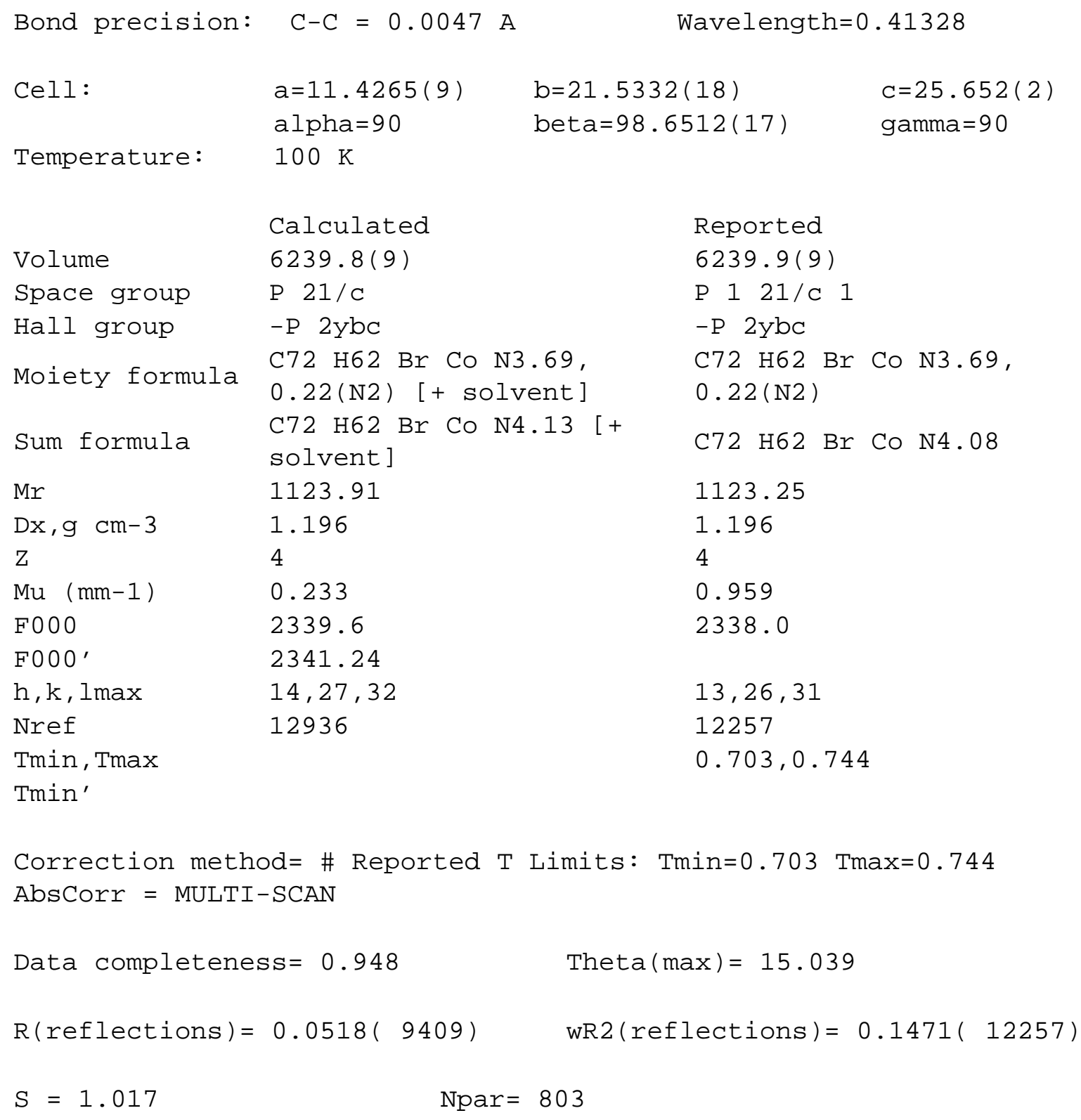


The following ALERTS were generated. Each ALERT has the format

test-name_ALERT_alert-type_alert-level.

Click on the hyperlinks for more details of the test.

Alert level $B$

PLAT029_ALERT_3_B _diffrn_measured_fraction_theta_full value Low . 0.952 Why?

\begin{abstract}
Author Response: Data was collected at a synchrotron source as a series of phi scans and this is the completeness that was obtained by this acquisition sequence. The geometry of the experiment (cryostat, LED fiber optic, detector, and beam path) prevented acquisition of omega scans.
\end{abstract}

Alert level C

PLAT077_ALERT_4_C Unitcell Contains Non-integer Number of Atoms .. PLAT220_ALERT_2_C NonSolvent Resd 1 C Ueq(max) / Ueq(min) Range PLAT222_ALERT_3_C NonSolvent Resd 1 H Uiso(max)/Uiso(min) Range PLAT260_ALERT_2_C Large Average Ueq of Residue Including N1N
Please Check
5.5 Ratio
6.2 Ratio
0.173 Check

Alert level $\mathrm{G}$

FORMU01_ALERT_1_G There is a discrepancy between the atom counts in the _chemical_formula_sum and_chemical_formula_moiety. This is usually due to the moiety formula being in the wrong format. Atom count from_chemical_formula_sum: C72 H62 Br1 Co1 N4.08

Atom count from_chemical_formula_moiety:C72 H62 Br1 Co1 N4.13

FORMU01_ALERT_2_G There is a discrepancy between the atom counts in the

_chemical_formula_sum and the formula from the _atom_site* data.

Atom count from_chemical_formula_sum:C72 H62 Br1 Co1 N4.08

Atom count from the _atom_site data: C72 H62 Br1 Co1 N4.13

ABSMU01_ALERT_1_G Calculation of _expt1_absorpt_correction_mu

not performed for this radiation type.

CELLZ01_ALERT_1_G Difference between formula and atom_site contents detected.

CELLZ01_ALERT_1_G ALERT: check formula stoichiometry or atom site occupancies.

From the CIF:_cell_formula_units_Z 4

From the CIF: _chemical_formula_sum C72 H62 Br Co N4.08

TEST: Compare cell contents of formula and atom_site data

$\begin{array}{lcrr}\text { atom } & Z^{\star} \text { formula } & \text { Cif sites diff } \\ \text { C } & 288.00 & 288.00 & 0.00 \\ \text { H } & 248.00 & 248.00 & 0.00 \\ \text { Br } & 4.00 & 4.00 & 0.00 \\ \text { Co } & 4.00 & 4.00 & 0.00 \\ \text { N } & 16.32 & 16.52 & -0.20\end{array}$

PLAT002_ALERT_2_G Number of Distance or Angle Restraints on AtSite

PLAT003_ALERT_2_G Number of Uiso or Uij Restrained non-H Atoms ...

PLAT041_ALERT_1_G CalC. and Reported SumFormula Strings Differ

PLAT083_ALERT_2_G SHELXL Second Parameter in WGHT Unusually Large

PLAT092_ALERT_4_G Check: Wavelength Given is not $\mathrm{Cu}, \mathrm{Ga}, \mathrm{Mo}, \mathrm{Ag}$, In $\mathrm{Ka}$

PLAT172_ALERT_4_G The CIF-Embedded .res File Contains DFIX Records

PLAT175_ALERT_4_G The CIF-Embedded .res File Contains SAME Records

PLAT176_ALERT_4_G The CIF-Embedded.res File Contains SADI Records

PLAT177_ALERT_4_G The CIF-Embedded .res File Contains DELU Records

PLAT178_ALERT_4_G The CIF-Embedded .res File Contains SIMU Records

PLAT301_ALERT_3_G Main Residue Disorder .............. (Resd 1 )

PLAT302_ALERT_4_G Anion/Solvent/Minor-Residue Disorder (Resd 2)

PLAT304_ALERT_4_G Non-Integer Number of Atoms in ..... (Resd 1 )

18 Note

17 Report

Please Check

5.32 Why ?

0.41328 Ang.

4 Report

1 Report

1 Report

2 Report

3 Report

$10 \%$ Note

$100 \%$ Note

139.69 Check 


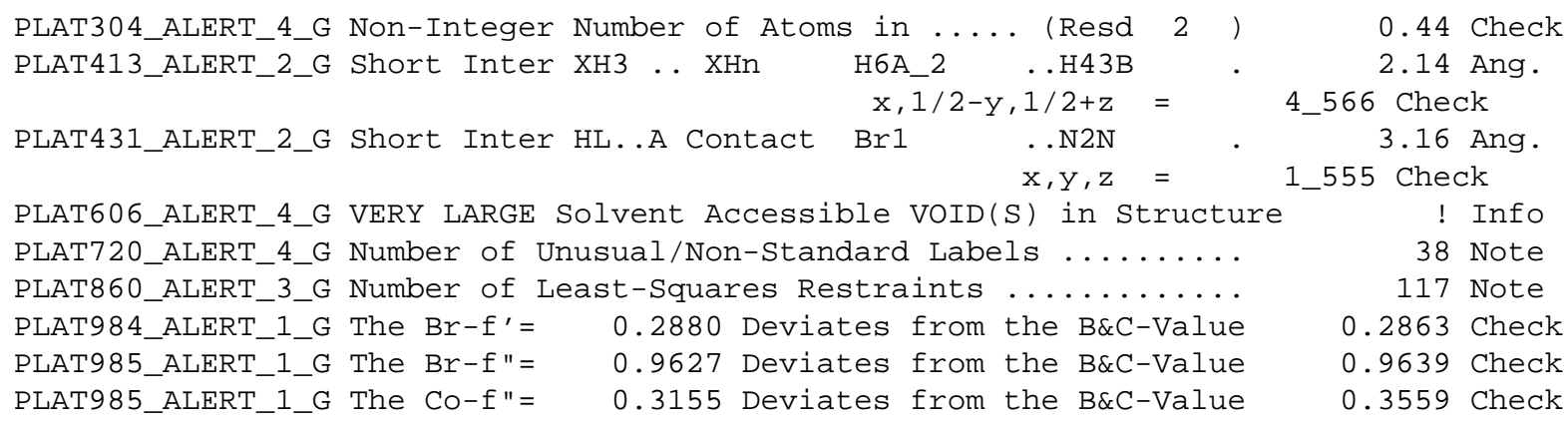

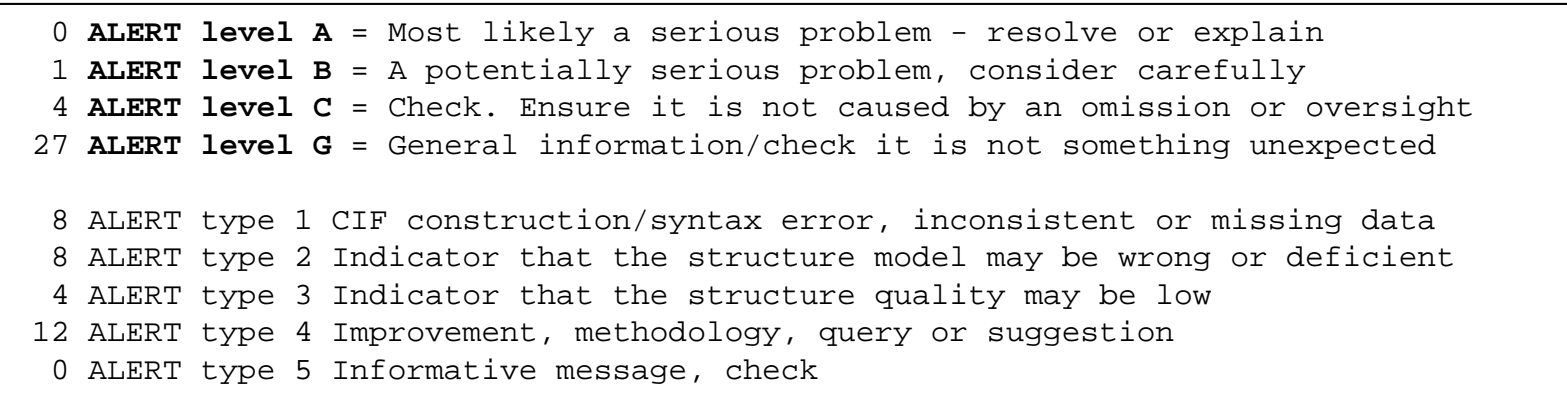

It is advisable to attempt to resolve as many as possible of the alerts in all categories. Often the minor alerts point to easily fixed oversights, errors and omissions in your CIF or refinement strategy, so attention to these fine details can be worthwhile. In order to resolve some of the more serious problems it may be necessary to carry out additional measurements or structure refinements. However, the purpose of your study may justify the reported deviations and the more serious of these should normally be commented upon in the discussion or experimental section of a paper or in the "special_details" fields of the CIF. checkCIF was carefully designed to identify outliers and unusual parameters, but every test has its limitations and alerts that are not important in a particular case may appear. Conversely, the absence of alerts does not guarantee there are no aspects of the results needing attention. It is up to the individual to critically assess their own results and, if necessary, seek expert advice.

\section{Publication of your CIF in IUCr journals}

A basic structural check has been run on your CIF. These basic checks will be run on all CIFs submitted for publication in IUCr journals (Acta Crystallographica, Journal of Applied Crystallography, Journal of Synchrotron Radiation); however, if you intend to submit to Acta Crystallographica Section C or E or IUCrData, you should make sure that full publication checks are run on the final version of your CIF prior to submission.

\section{Publication of your CIF in other journals}

Please refer to the Notes for Authors of the relevant journal for any special instructions relating to CIF submission.

\section{PLATON version of 22/04/2020; check.def file version of $09 / 03 / 2020$}


